\title{
THE EXPERIENCES OF PEOPLE WHO RE-ENTER THE WORKFORCE FOLLOWING DISCHARGE FROM A FORENSIC HOSPITAL
}

A thesis submitted to Auckland University of Technology in partial fulfilment of the degree of Masters of Health Science at Auckland University of Auckland.

Jenni Tregoweth 


\section{TABLE OF CONTENTS}

Attestation of Authorship.....................................................

Acknowledgement...........................................................

Ethics Approval.....................................................II

Abstract..............................................................

Key to Transcripts................................................

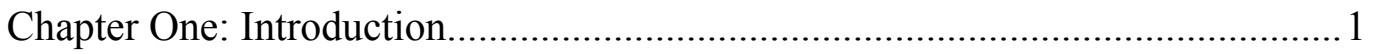

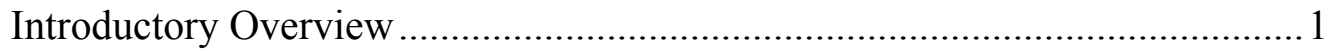

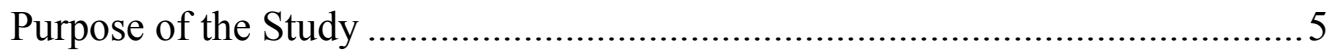

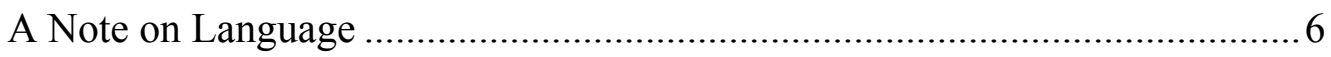

My Background and Arriving at the Research Question ................................ 8

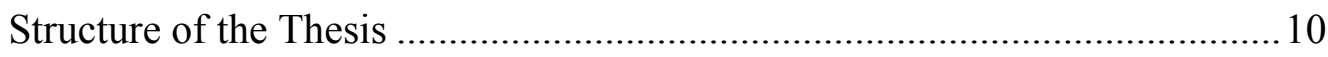

Chapter Two: Contextualising the Study ……................................................ 12

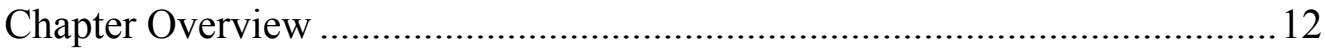

Vocational Models in Mental Health Rehabilitation ...................................... 13

Traditional Approaches to Work in Mental Health....................................... 13

Supported Employment Approach .......................................................... 14

Multidimensional Approach to Vocational Opportunities .......................... 15

Vocational Rehabilitation Models: The International Experience............... 16

Meaningful Employment for People with Psychiatric Disabilities................. 17

Meaningful Work: Relationship to Recovery …........................................ 18

Meaningful Work: Relationships to Other Life Domains........................... 19

Meaningful Work: Relationship to Job Selection ........................................ 19

Meaningful Work: Relationship to Job Tenure.........................................20

Meaningful Work: Relationship with Disclosure ..................................... 22

Work as a Dynamic Component of Mental Health Rehabilitation Practise.....23

Vocational Rehabilitation: The Forensic Psychiatry Perspective ...................25

Forensic Community Rehabilitation: A Vocational Perspective .................25

Rehabilitation and Work in Forensic Psychiatry: The Literature ...............26

Risk Management: Vocational Rehabilitation Perspective.........................27

Forensic Psychiatry: A Cultural Perspective..............................................28

Work Issues in Forensic Psychiatric Rehabilitation: A Summary ...............29

Review of the New Zealand Literature: Mental Health and Work ...................30 
The New Zealand and International Experience: A Socio-political View........34

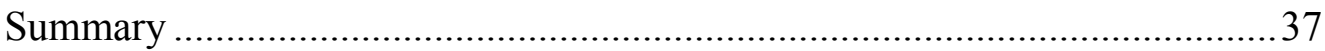

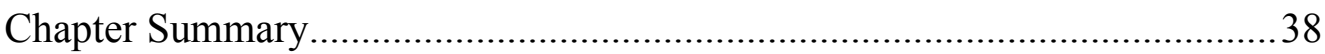

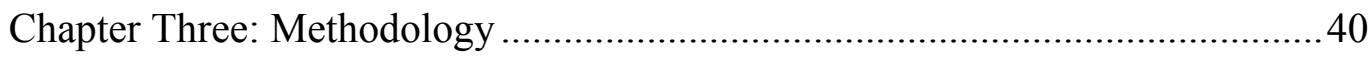

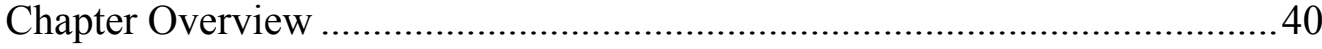

Selecting a Critical Hermeneutic Perspective ................................................. 41

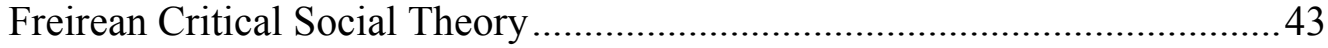

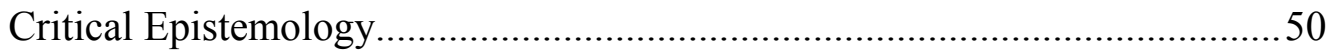

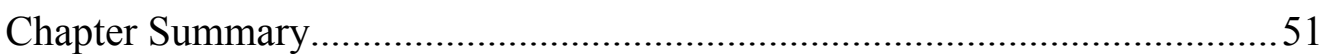

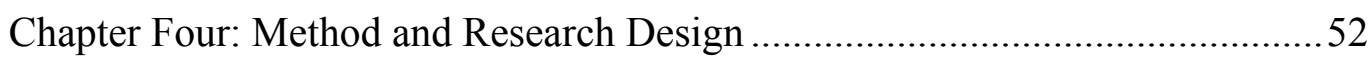

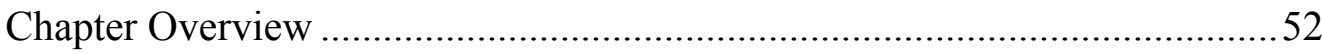

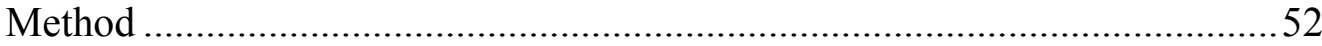

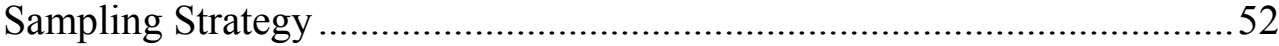

Intermediaries and the Recruitment Process .............................................55

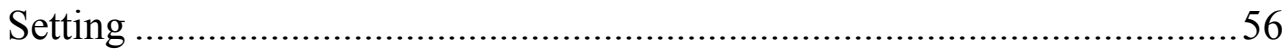

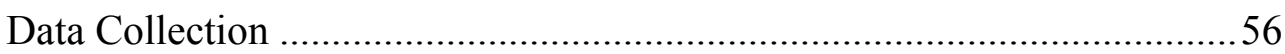

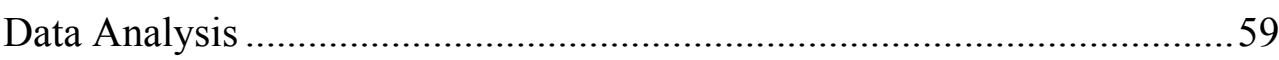

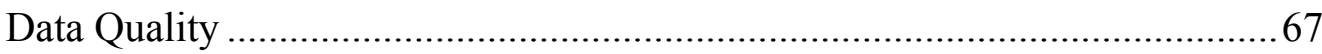

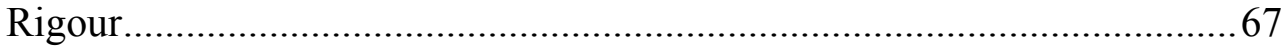

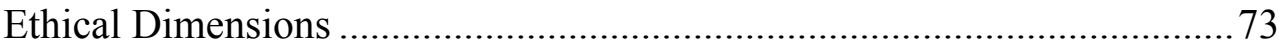

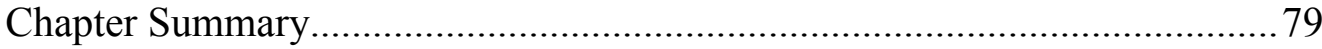

Chapter Five: The Liberatory Quest: From Alienation to Inclusion and

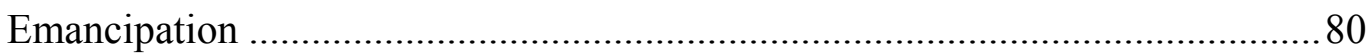

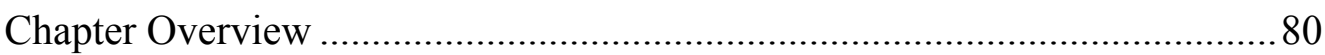

Overarching Theme: Re-entering the World............................................. 84

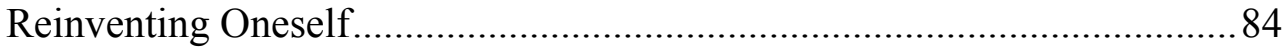

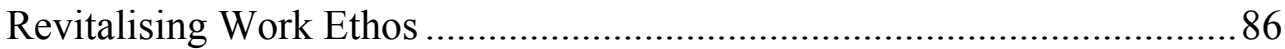

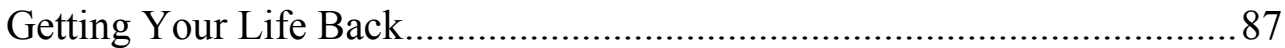

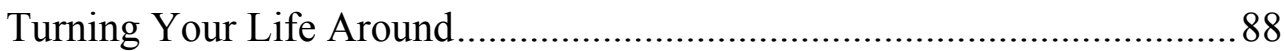

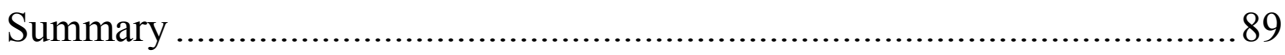

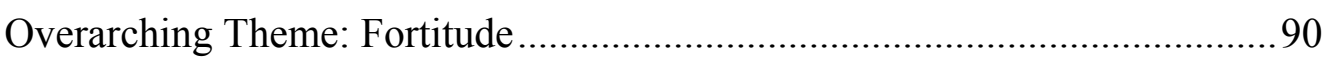

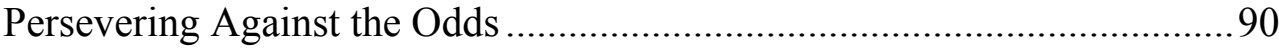

Navigating through the Complexities of Disclosure …...............................92

Dealing with Unwanted Disclosure ...........................................................96 


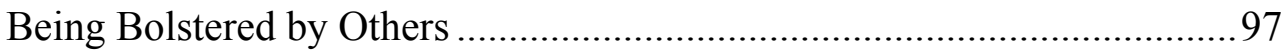

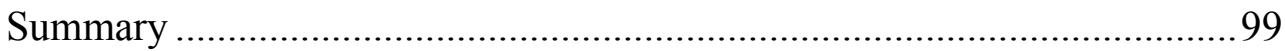

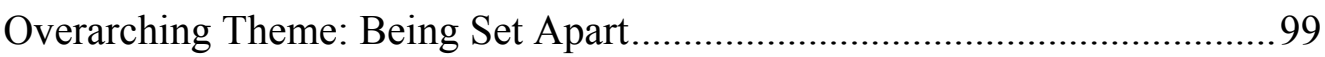

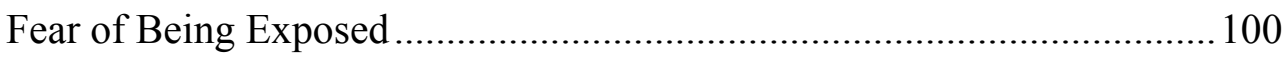

Being Alienated from Others .............................................................. 102

Keeping Colleagues at Arms Length ...................................................... 104

Being Scrutinised and Judged on the Basis of Diagnosis ......................... 104

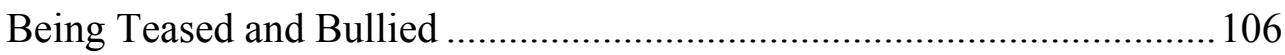

Keeping Safe: Staying Right Away from Drugs and Alcohol .................. 108

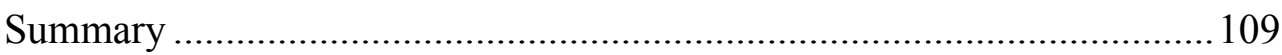

Overarching Theme: Redeeming Oneself.................................................110

Re-establishing Family Pride ............................................................. 110

Others Believing in You vis-à-vis Believing in Yourself..........................112

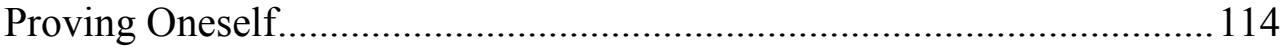

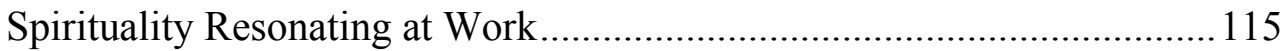

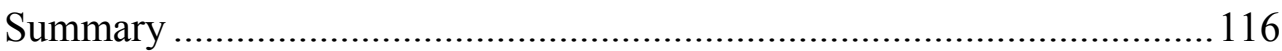

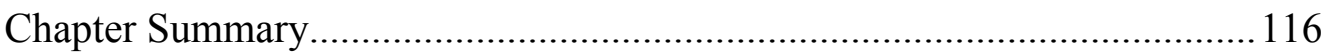

Chapter Six: Authentic Reflection: Being Grounded in the Here and Now, Within

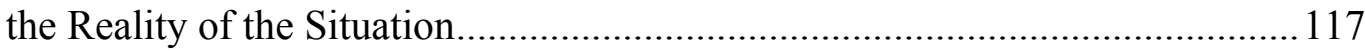

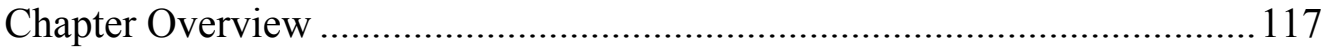

Overarching Theme: Doing the Ground Work ...........................................119

Building Work Skills and Resources ......................................................119

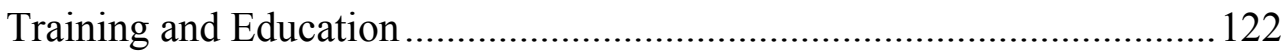

Others Making Re-entry to Work More of a Level Playing Field ............. 124

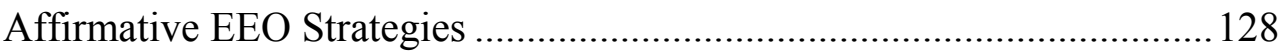

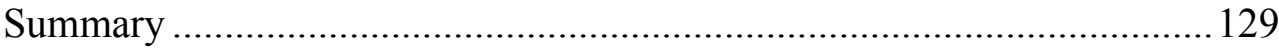

Overarching Theme: Weighing-up the Economics......................................130

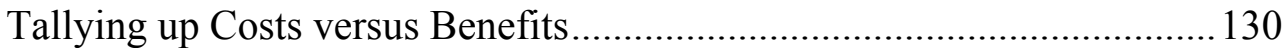

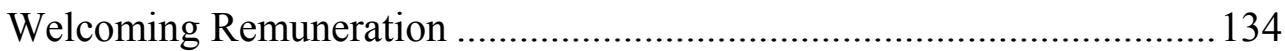

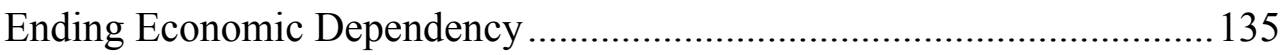

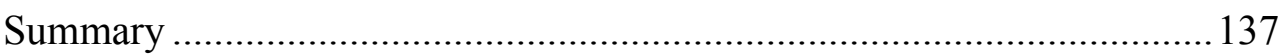

Overarching Theme: Fitting in with Workaday Life ..................................137

Becoming Versed in Workplace Norms \& Conditions.............................. 138

Casualisation of Work Conditions .............................................................. 141 
Being Acknowledged: Rewards and Promotion ...................................... 142

Mastering Work Skills ......................................................................... 144

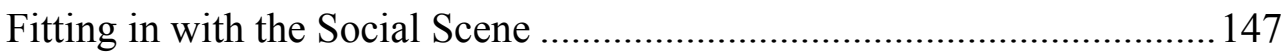

Getting On-the-Job Support ............................................................ 149

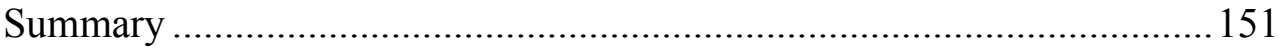

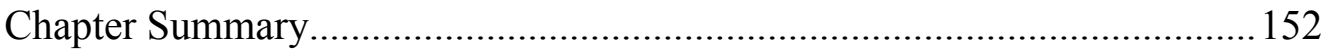

Chapter Seven: Humans as Unfinished and Incomplete; within a Likewise

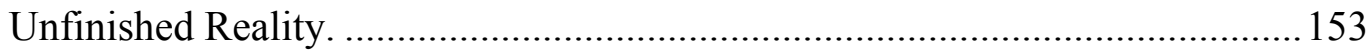

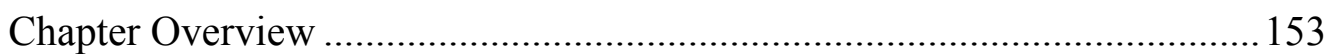

Overarching Theme: Getting-Going on Workday Mornings........................155

Medication Induced Lethargy and Brain Fog ......................................... 156

Adjusting to New Worker Routines...................................................... 159

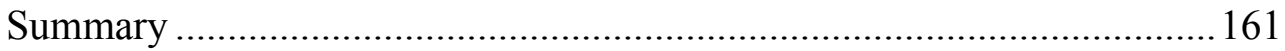

Overarching Theme: Experiencing the Physical Self at Work .....................162

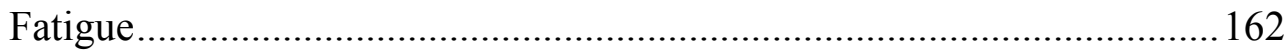

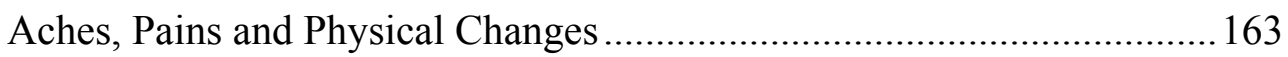

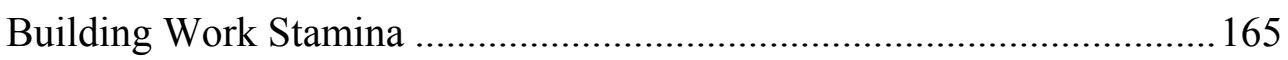

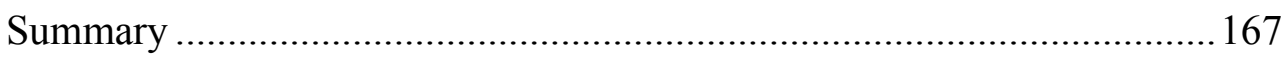

Overarching Theme: Self-Regulating Features of Illness at Work................168

Discerning Symptoms of Anxiety versus Workplace Stress .................... 168

Managing Hallucinations and Delusional Thoughts ...............................170

Being Inattentive and Preoccupied........................................................... 171

Formulating Wellness-at-Work Strategies............................................. 172

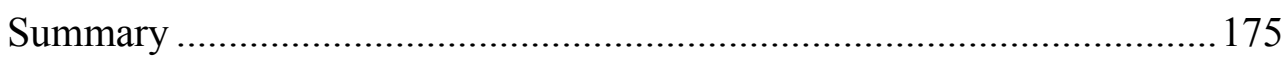

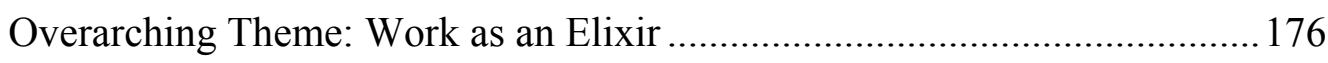

Being Bolstered by Personal Accomplishment.........................................176

Realising Career and Work Aspirations....................................................177

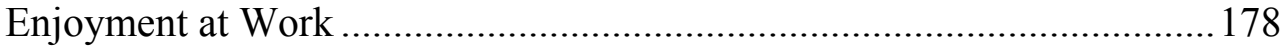

Contributing Back to the Community .......................................................179

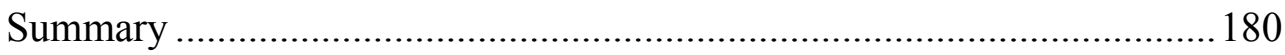

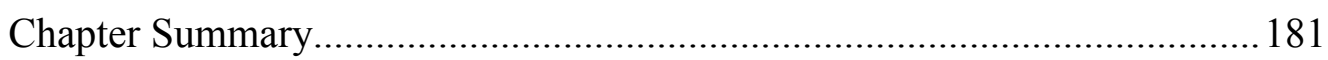

Chapter Eight: Discussions and Conclusions................................................... 182

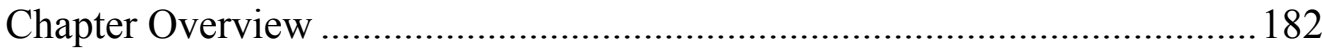

Returning to Work: Summary of the Findings..............................................182 
The Relevance of the Critical Perspective 187

Forensic Vocational Rehabilitation: Implications for Practise 190

Vocation as an Integral Part of Forensic Psychiatry Rehabilitation Practise 190

Doing the Ground Work …................................................................ 191

Enhancing Worker's Success and Satisfaction ........................................193

Interconnections with Social Networks ................................................. 194

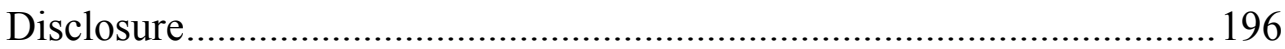

Getting Going on Work Day Mornings ....................................................198

Wellness-at-Work Plans......................................................................... 198

Creating Employment Opportunities: Socio-political Interventions..........201

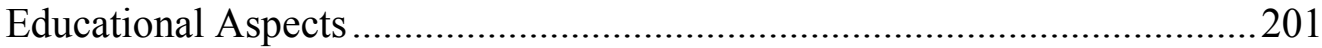

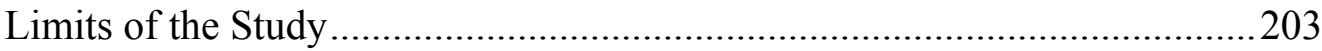

Suggestions for Future Research...........................................................205

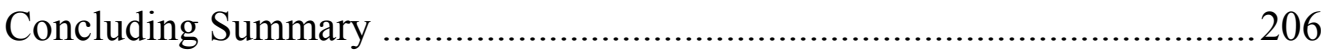

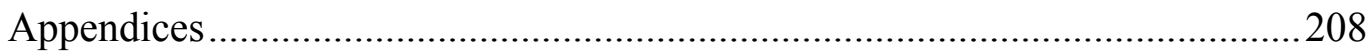

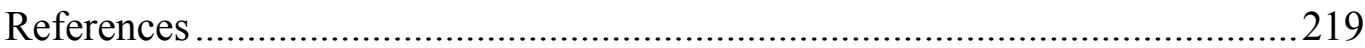


ATTESTATION OF AUTHORSHIP

I hereby declare that this submission is my own work and that, to the best of my knowledge and belief, it contains no material previously published or written by another person more material which to a substantial extent has been extended has been accepted for qualification of any other degree or diploma of a university or other institution of higher learning, except where due acknowledgement is made in the acknowledgements.

Signed: 


\title{
ACKNOWLEDGEMENTS
}

These research endeavours were a shared journey with the eight people who offered their experiences so that we may all learn from them. I acknowledge the informants for their generosity, courage and hopefulness.

I have reached this destination because of the contributions and support from others; a sentiment expressed in this whakatauki.

\author{
Ko koe ki tena \\ Ko au ki tenei \\ Kiwai o te kete
}

You at that

Me at this

Handle of the basket.

My supervisors, Professor Jo Walton and Kirk Reed, have wisely guided this journey through their astute blend of thought-provoking gentle challenges and warm support.

Supportive friends and colleagues have contributed by sharing the load in many ways. Kerry, Bill, Francesca, Alison, Mary, Stephanie, Janene, Annette, Brian and Lizzie all have my enduring gratitude.

My family, Lloyd, Margaret and Graeme Tregoweth impressed on me from my earliest memories, albeit sometimes rather relentlessly, that learning is a life long quest. My children, Jody, Lara and Joseph Cavit, have inspired my learning and encouraged this journey. 


\section{ETHICS APPROVAL}

Ethical approval for this research was granted by the Health and Disability Ethics Committee.

Application number: AYK/04/11/303

Date of approval: $\quad 16.12 .04$ 


\section{THESIS ABSTRACT}

This critical hermeneutic study explored what it is like to re-enter the workforce following long-term forensic hospitalisation. An in-depth analysis of the phenomenon was completed, with the aim of evoking insights and developing understandings about the lived return-to-work experience. As this research was situated within the critical paradigm, the process of seeking, securing and sustaining employment was viewed in terms of power relationships, and through the multiple positionings of psychiatric disability, employment status and social capital.

An unstructured interview process was used to explore the return-to-work experiences of eight purposefully selected informants with a history of mental illness and prior illness-related offending. They were living in the community and had returned to part or full-time employment, which they had sustained for at least six months. The gathered data was interpreted using hermeneutic analysis. This process revealed a number of themes, which were clustered into related groups, under eleven essential overarching themes. Freire's (1972) critical social theory was used to add critical depth to the findings.

The findings reveal that returning to work exposes people who are affected by mental illness to an array of challenges and personal opportunities. People who have a forensic psychiatric history can encounter complex employment barriers related to stigma and misunderstanding. Therefore, the selected critical hermeneutic design provided a congruent framework with which to view the informants' quest to seek, secure and sustain employment. Despite significant obstacles, securing employment provides opportunities for individuals to test their skills while engaged in meaningful work activity. The acquisition of work skills can result in individuals' experiencing a strong sense of self-satisfaction. The experience of being bolstered by personal accomplishment often co-exists with, but is not necessarily negated by, difficulties that arise on-the-job.

As there is scant reference to forensic rehabilitation within the mental health vocational literature, this study may be a timely contribution. It may also be used to add depth to the knowledge base within the field of mental health 
rehabilitation, in particular the specialised areas of forensic rehabilitation and vocational practise. Therefore, it may be a positive precursor to further discussion and analysis regarding work and education outcomes from the unique forensic psychiatric perspective. 


\section{KEY TO TRANSCRIPTS}

The following conventions and abbreviations have been applied to the presentation of data from the informants in these research findings.

Names: $\quad$ All names used to refer to the informants in this study are pseudonyms. Excerpts from the informants' interviews are identified by page, which are referenced to the transcription.

Italics: Where excerpts of the interview transcripts are included, the informants' words are italicised.

[Square brackets] These brackets indicate that identifying features such as a staff member or place name has been removed for reasons of confidentiality. These include hospitals, worksites and specific work/ professional occupations.

(Parentheses) When included in the interview excerpts, these indicate the researcher's own words.

These indicate a pause contained in the original audiotaped interview.

../.. When this symbol is included in the interview excerpts it indicates that material has been edited out. 


\title{
CHAPTER ONE: INTRODUCTION
}

\author{
Introductory Overview
}

A strong tradition of using work as therapy has prevailed within psychiatric hospitals for over six hundred years, with some of the earliest historical evidence showing that work programmes existed in Spain since the first mental hospitals were founded in the 15th century (McCurrin, 1994; Schneider, 1998). In recent years there has been a move towards supported employment, ${ }^{1}$ a concept which originated in North America in the 1980s. This approach promoted the premise of forgoing the stepwise prevocational 'train and place' methods in favour of direct placement with on-the-job follow-up. Since the early nineties, this concept has received increasing support from mental health researchers (Becker, Drake \& Naughton, 2005; Bedell et al., 1998; Bond, Drake, Mueser \& Becker, 1997; Bond, 1998; Bond, 2004; Lehman, 1995; Schneider, 1998). The purpose of this thesis is to reveal the experiences of people who are returning to open employment $^{2}$ following their discharge from a forensic hospital. In this introduction, mental health vocational rehabilitation endeavours are positioned within a historical and socio-political context. A brief overview of the critical hermeneutic design includes reference to Freirean critical theory which informs this study. Drawing on the body of knowledge within the mental heath rehabilitation literature, the inherent challenges and benefits of work are outlined, with particular focus on the unique forensic psychiatric perspective.

Work has been found to be a strong predictor of the successful community adjustment of people with psychiatric disabilities ${ }^{3}$ (Gioia, 2005; Sundar \&

\footnotetext{
${ }^{1}$ Supported employment has been defined in the New Zealand literature as: "The process of placement, training and ongoing support of people with disability aimed at their earning financial remuneration in integrated work settings" (Association for Supported Employment in New Zealand, 1996, p. 3).

${ }^{2}$ Open employment aka competitive employment is defined as: "Community jobs that any person can apply for, in regular places of business, paying at least minimum wage, with mostly nondisabled co-workers" (Bond, 2004, p. 346).

3 Anthony, Cohen, Farkas and Gagne (2002) employ the term 'people with psychiatric disabilities', defining this population as: "Persons with psychiatric disability have diagnosed mental illnesses that limit their capacity to perform certain tasks and functions (e.g. interacting with family and friends, interviewing for a job) and their ability to perform in certain roles (e.g. worker; student). (p. 4)
} 
Ochocka, 2004). These individuals, however, have to overcome substantial barriers in seeking and securing employment. Stigmatisation is a significant barrier that often impedes the success of mental health vocational efforts, as the handicapping effects of stigma is often more limiting than the disability itself (Bybee, Mowbray \& McCrohan, 1996; Dorio, 2004; Garske, 1999). When people who have psychiatric disabilities have also been engaged with forensic psychiatric services, they have even less access to the existing range of work opportunities on offer (Joe, 2003). As this group's job search endeavours are strongly influenced by socio-political forces, this thesis uses a critical lens to explore the research question: "What are the return-to-work experiences of people following long term hospitalisation in a forensic setting?"

In forensic psychiatry ${ }^{4}$, rehabilitation strategies and vocational interventions coexist alongside the more traditional interventions of medication, risk assessment and basic support (Couldrick \& Aldred, 2003). When people move from a forensic hospital back into the community, the prior inpatient rehabilitation focus on developing their readiness for work is complemented by intensive forensic community follow-up. This mandated support involves assertive case management that is centred on symptom reduction and rehabilitative and risk management strategies. These are interventions that maximize both the individual's mental wellness and their potential to obtain and sustain satisfying employment. Although often 'ready and willing' prospective employees, people with a forensic history have to contend with the dual stigma of having a psychiatric illness and a history of illness-related offending. Hence, people may have the required work skills and career aspirations, but feel unable to confront the difficulties in dealing with potential discrimination from employers (Lapsley, 2003; Mental Health Commission, 1999; Joe, 2003).

\footnotetext{
${ }^{4}$ Forensic Psychiatry is the branch of psychiatry which requires special knowledge and training in the law as it relates to the mental state of the offender, or alleged offender, and training and experience in the assessment, treatment and care, including care in the community, of persons who have offended or are alleged to have offended, or appear likely to offend because of their psychiatric condition. (Mason, 1988, p. 3)
} 
There is an increasing volume of evidence about the importance of work within the mental health rehabilitation literature. In illuminating the Psychiatric Rehabilitation approach to work, Boston University's Center of Psychiatric Rehabilitation provides much of the evaluative research that informs this returnto-work study's theoretical underpinnings. There is, however, little written about the experience of work from a forensic psychiatric standpoint. In addition, authors writing from the forensic perspective concur that there is scant reference to rehabilitation, or vocational endeavours, within the forensic literature (Couldrick \& Aldred, 2003; Lindqvist \& Skipworth, 2000; Lloyd, 1999; Tracey, 2000). With increasing professional requirements for practise to be validated by evidence, the deficit of vocational and rehabilitation literature in relation to actual practise within forensic settings is of concern. In addition, there is an absence of qualitative studies illustrating the lived experience of people who are recipients of forensic psychiatric services. This means that their unique perspective on what constitutes meaningful rehabilitation and their notions about which interventions support their community readjustment and continued recovery, remains unexplored. Hence, the lived experiences of people who were returning to work following their discharge from a forensic secure-care facility was a phenomenon I was drawn to explore in more depth.

People with psychiatric disabilities prioritise employment; money and opportunities for social participation as the interventions that promote the greatest health gains (Sayce, 2000). Work has been found to provide a distraction from disability, with the person's shift in consciousness from illness to work issues enhancing and supporting the recovery process $^{5}$ (Krupa, 2004; Rogers, Anthony, Cohen \& Davis, 1997; Schneider, 1998). From a service provider perspective, employment appears to protect the worker against the return of symptoms, thereby reducing recidivism and their usage of mental health services (Becker, Drake \& Naughton; 2005; Blankertz \& Robinson, 1996; Schneider,

\footnotetext{
5 "Recovery is a deeply personal, unique process of changing one's attitudes, values, feelings, goals, skills and/or roles. It is a way of living a satisfying, hopeful and contributing life, with or without limitations caused by the illness. Recovery involves the development of new meaning and purpose on one's life as one grows beyond the catastrophic effects of a mental illness". (Anthony et al., 2002, p. 31)
} 
1998). Individuals surveyed by Mallik, Reeves \& Dellario (1998), however, identified financial resources, employment resources and vocational skills as the areas that still present the greatest barriers to community integration. Despite many diverse educational backgrounds; work aptitudes; career aspirations and previous work history, internationally people with psychiatric disabilities have unemployment rates that often exceed 85 percent (Sayce, 2000; Tsang, 2000).

Clearly, vocational rehabilitation does not exist in isolation; it must interact with the politically driven labour market as well as the wider community. In contrast to the proactive legislative measures in North America and some European countries (Schneider, 1998), New Zealand has, on a variety of levels, traditionally failed to fully embrace the issues related to employment for people with disabilities (Lapsley, 2003; Mental Health Commission, 1999). In 2003, despite a rapidly decreasing general unemployment rate, significantly more people with disabilities were unemployed (an increase of $19.5 \%$ over five years), and complained about discrimination than in the mid 1990s (Mintrom \& True, 2004). This acknowledges people with disabilities to be one of the most disadvantaged groups in the current New Zealand employment environment (Mintrom \& True, 2004). The discriminatory practices of some employers and workers can create even more complex employment barriers for people disabled by mental illness, particularly for those with a forensic history (Mental Health Commission, 1999). Therefore, with the population under study, an individual's preadmission work history, capabilities and desire to work, are often subsumed by stigma, psychiatric diagnosis and general underemployment.

The issues of stigma and discrimination within employment are present at all stages of re-entry to work, and appear at all organisational levels, but the form that these barriers take is frequently surprising and often unpredictable. In mental health vocational rehabilitation practise, the inherent challenges and contradictions are often difficult to prepare for. This is because the subjective, obscure socio-political nature of some of these complexities makes them difficult to discover and name. The community reintegration process for people with a forensic history is heavily influenced by a risk minimisation approach. Vocational rehabilitation practitioners, however, also have an important role in 
trying to change attitudes within the employment sector to develop social inclusion and to challenge discriminatory practises (Sundar \& Ochocka, 2004).

I selected a critical hermeneutic design as I perceived it was the 'best fit' with the research question, and the associated socio-political context. I then recorded the subjective experiences of people who had returned to work while navigating through the complexities of having a history of mental illness and illness-related offending. In line with a critical inquiry, I utilised Freire's constructs to critique my findings in order to add critical depth and explicate the social political influences. Accordingly, the ensuing insights and understandings about the return-to-work experience are related to both forensic psychiatric rehabilitation and mental health vocational practise.

\section{Purpose of the Study}

The purpose of this study was to gain insights and develop understandings about the lived experience of returning to work following long-term hospitalisation in a forensic setting. A purposive sample of eight people, who had a history of psychiatric disability and illness-related offending, joined this study as informants. As people who have a forensic psychiatric history encounter many complex employment barriers related to stigma and misunderstanding, a critical hermeneutic design was selected. In line with the requirements of a critical design, this study aimed to enhance the informants' understanding of the world, and the way it is shaped, in relation to the politics of employment and related community discrimination (Burns \& Grove, 1997).

Both the research question and design align with the employment directions outlined in the innovative New Zealand Disability Strategy (Minister for Disability Issues, 2001). The strategy has helped to raise general awareness of disabilities, and has consequently also exposed the forces of hegemony and discrimination within society. This return-to-work study endeavoured to support the strategy's intent, which is to promote employer acceptance and create meaningful employment options for people with disabilities, including psychiatric disabilities. By revealing insights and understandings about the 
return-to-work experience, this study sought to inform both forensic and vocational rehabilitation practise. It is my hope that the findings will evoke further discussion and analysis regarding work and education outcomes from the unique forensic psychiatry perspective. Potentially, the ensuing discussions may contribute to a shift within forensic services' programme development and research agendas, from a predominantly risk management focus, to one which is more inclusive of rehabilitative and vocational outcomes.

\section{A Note on Language}

While mental health care has been a rapidly evolving paradigm over the last two decades, so too has the language. Within the practise of mental health rehabilitation, using diagnostic categories such as "schizophrenic" to describe people is clearly regarded as outdated and inappropriate. The shift away from terms such as "psychiatric patient" has seen the language evolve and change over time. Terms such as "mental health consumer", "service user", and "psychiatric survivor" have been used at different times, in different circumstances. While the debate over the correct terminology continues, there is now consensus within the general disability sector that using 'people first' language is the most appropriate. Researchers from the Center of Psychiatric Rehabilitation at Boston University, who are major contributors to the body of Psychiatric Rehabilitation knowledge, use the term, "persons who have experienced a severe psychiatric disability", shortened to "persons with a psychiatric disability" (Anthony et al., 2002). In relation to this return-to-work study, this description had its merits as it distinguishes this population under study as people who have been diagnosed with a serious mental illness such as schizophrenia or bipolar affective disorder, rather than people who are psychologically distressed. This terminology is therefore used within this thesis in specific relation to the body of the psychiatric rehabilitation knowledge.

After posing the question to the informants about what language should be employed when both analysing and reporting this study's findings, a joint decision led to using the terms coined by the New Zealand Mental Health Commission, "people who are affected by mental illness", which is abbreviated 
to "people with mental illness". Within the New Zealand mental health sector, the Maori term, "tangata whaiora", which means people who are seeking wellness, is now frequently used within a cultural context. Therefore, "tangata whaiora" is used in this study when this fits best within the cultural context being described.

While "people with mental illness" is used most often to describe the population under study, when the context has required a more detailed term, I have used, "people with mental illness and prior illness-related offending". While a lengthy phrase, it adheres to the principles of 'people-first language' and was developed in consultation with the informants during the member check session. Terms such as "service user", "consumer", "client", "resident" (as pertains to the informants' prior forensic inpatient status) are also used sparingly in the text depending upon the role being described, and in some instances, the quote being used. As this study is situated within the critical paradigm, the people who were interviewed are called "informants" as they are seen as research collaborators who inform this study, rather than people who merely participate in a research process. The exception to this is the participant information sheets that were designed to conform to the Ministry of Health Regional Ethics Committee guidelines.

Within the practise of mental health rehabilitation, many health professional groups are represented. These groups include occupational therapists, social workers, mental health nurses and mental health support workers. Within the body of the thesis, the term used most often is the generic title of mental health practitioner. When there are specific references to professional groups, they are referred to by title. In differentiating the professionals who work in the specialist area of mental health vocational rehabilitation, the term vocational practitioner is used to describe the occupational therapists and other professionals who work in this specialist area of mental health practise. While the term 'forensic' is used to describe different aspects of the legal process, in this thesis it always pertains to the area of forensic psychiatry. 
My Background and Arriving at the Research Question

My lengthy career as a mental health practitioner commenced with my graduation as an Occupational Therapist in the late 1970s. During the earlier part of my career, I worked in many diverse areas within the mental health sector. Since the late 1980s, however, my practise focus has increasingly been on the area of rehabilitation within the clients' learning and working environments. My professional interest in work and education outcomes for people with mental health disabilities aligns with the prevailing view held by the politically led mental health consumer movement, that meaningful employment is believed by consumers to be essential to the recovery process (Deegan, 1992).

In 2000, my interest in the international mental health rehabilitation perspective led me to enrol in the inaugural postgraduate certificate in mental health rehabilitation at the Auckland University of Technology (AUT University). This module was taught by Dr Marianne Farkas who is the co-principal investigator from the Center of Psychiatric Rehabilitation, Boston University. I was profoundly inspired by the breadth of Marianne's research and practise-based knowledge. During her presentations, Marianne highlighted the interrelationships between these mental health constructs and the prevailing socio-political influences. Her teaching further impressed upon me that vocational rehabilitation always exists in a socio-economic, cultural and political context. In addition, the research based psychiatric rehabilitation technologies, which are underpinned by the principles and tenets of the Psychiatric Rehabilitation model, have become guiding motivations for my practise, vocational service development and this study (Anthony et al., 2002).

As my Masters studies progressed, I gradually identified that I wished to research the experiences of people who have had a major disruption to their employment history due to their previous forensic hospitalisation. To this end, I have framed the understanding of the return-to-work experiences within the critical paradigm, and thereby endeavoured to generate a deeper understanding of the informants' experiences within their socially, culturally, politically and historically conditioned context. My initial introduction to critical theories occurred in 1990 when I embarked on a Certificate of Women's Studies at the University of 
Auckland. As I completed this certificate over ten years, I found my world view to be increasingly influenced by the critical perspective; the paradigm in which feminist theory is predominately positioned. Writings from contemporary socialist feminists such as Shelia Rowbotham provided me with a theoretical framework with which to explore the interrelationships of economics, power and justice within society. The relationship of these concepts to my mental health practise was able to be further explored during my postgraduate study at AUT University.

I selected the population under focus for my study, because at the juncture of rejoining the community, people leaving a forensic inpatient setting have often progressed in their recovery journey to the point of being productive, work-ready employees. The return-to-work prospects for forensic clients are often more available in terms of work skills and abilities than for the general population of people with mental illness, but less available due to community fear and misunderstanding. During my lengthy vocational rehabilitation career, I have never experienced a violent workplace incident involving a client with whom I was either working with directly, or who was attending the vocational service I am involved with. Hence, I have a very positive view of community safety within the workplace based on my personal experience. As a mental health vocational practitioner, I am aware that issues of stigma and discrimination are present at all stages of the job search process, but the form that these barriers take is often unpredictable. Therefore, in vocational practise it is difficult to prepare for these discriminatory practises; hence it was a phenomenon I was drawn to explore in more depth.

Entering employment is an event which brings the person's history of mental illness and illness-related offending sharply into question, sometimes for the first time post discharge and often for the first time in a public arena. Therefore, returning to work is often a defining moment in people's recovery. In 2002, during a practise session that was related to my mental health rehabilitation studies, a client expressed the experience of returning to work after being in a forensic setting as "I'm getting my life back". I was intrigued to subsequently hear the same statement from others who had experienced a similar return-to- 
work journey, and this has proved to be a defining motivator for my study which focuses on the lived experience of this phenomenon.

As people with a forensic history encounter many complex employment barriers, including those related to stigma and misunderstanding, I chose a critical hermeneutic design for this study because of the 'fit' with the person's lived experience. The importance of providing opportunities for people with mental illness to have a voice within research is reflected in the following poetic description by Janet Frame (1992) who has provided an autobiographical account of New Zealand psychiatry in the fifties and sixties.

$$
\begin{aligned}
& \text { they died because the words they had } \\
& \text { spoken } \\
& \text { returned always homeless to them (p. 72) }
\end{aligned}
$$

The experience that is conveyed within these words has heavily influenced my selection of a research design that focuses on lived experience as a means of exploring the research question: "What are the return-to-work experiences of people following long-term hospitalisation in a forensic setting?"

\section{Structure of the Thesis}

This thesis is presented in eight chapters. Chapter One provides an introductory overview of work as it is portrayed within both mental health rehabilitation and New Zealand disability literature, with emphasis on the forensic psychiatric perspective. In presenting the background to this study, which is situated within the critical paradigm, work is viewed as both a therapeutic tool and as a right of citizenship. Accordingly, the introduction serves to position this study within a historical and socio-political context. This chapter also outlines the purpose of this study, the language used to describe people and roles, and how I arrived at the research question.

In Chapter Two, the study is contextualised within the body of the existing knowledge. In expanding on the overview of the literature provided in Chapter One, current and significant research into work, within both a mental health rehabilitative and socio-political context, is reviewed and critiqued. These 
research findings are linked to current New Zealand employment literature and vocational rehabilitation within the specialist area of Forensic Psychiatry.

Chapter Three discusses the critical hermeneutic research methodology which underpins this study. Accordingly, Freire's critical social theory is described, including specific explanations of the Freirean terminology used to explicate this study's findings.

Chapter Four details the research methods employed in this study, describing the data analysis used to reveal the emerging themes. This chapter then describes how the rigour and ethics requirements have been addressed since this study's inception.

In Chapters Five, Six and Seven, the study findings are presented and discussed. Each of these chapters has been assigned a Freirean phrase as a title. This phrase encapsulates the essence of the emerging themes and ensuing discussion within each chapter. Specifically, Chapter Five focuses on the 'liberatory quest: from alienation to inclusion and emancipation'. Chapter Six then looks at 'authentic reflection: being grounded in the here and now, within the reality of the situation'. Chapter Seven concludes the three findings chapters and is titled 'humans as unfinished and incomplete; within a likewise unfinished reality'. The meaning of each of these Freirean title phrases, and their relationship to the study's findings, are explained in the respective chapters. In each of these findings chapters, both the informants' words and Freire's critical theory constructs are used to add depth and meaning to the discussion associated with each of the emerging themes.

In concluding this thesis, Chapter Eight summarises and then integrates the study's findings by detailing the implications for forensic psychiatry and vocational rehabilitation practise. In addition to identifying the limitations of this study and future research directions, the concluding comments also address the educational needs of vocational rehabilitation practitioners. 


\section{CHAPTER TWO: CONTEXTUALISING THE STUDY}

\section{Chapter Overview}

This literature review seeks to contextualise this study by placing it within the context of the body of knowledge. In addition, this review seeks to highlight gaps in the literature and provide a rationale for completing this study. Since the deinstitutionalisation of New Zealand's psychiatric hospitals commenced in the early nineties, the changing context of mental health care has been driven by the principles of community integration. Consequently, both nationally and internationally, employment in the open workforce has become a more available option for people with psychiatric disabilities (Lapsley, 2003; Mental Health Commission, 1999). This literature review extends back over the last sixteen years in order to encompass the period since this major mental health paradigm shift towards community integration.

This chapter provides a comprehensive overview of work within the context of forensic psychiatry and mental health rehabilitation. Initially, this review commences with a description of mental health vocational models, from both a historical and international perspective. The subsequent paragraphs are grouped under the heading of 'meaningful work', and they explore the interrelationship of this concept with: the 'recovery' approach; other life domains; job selection; job tenure and disclosure. The following section positions vocation as an integral element of mental health rehabilitation practise. The unique forensic perspective is then explored, with emphasis on the rehabilitative versus risk management approaches to vocational rehabilitation and job placement. As there is scant reference to employment-related issues within the New Zealand mental health literature, the subsequent national overview largely relies on government publications, the popular press and conference publications. In accordance with the critical hermeneutic design of this study, this chapter is drawn to a conclusion by critically examining the socio-political and cultural influences on the work prospects of people with mental illness. In comparing the international evidence with the socio-political issues identified in the New Zealand literature, the 
relationships between the labour market, the political climate and the vocational needs of people with psychiatric disability are highlighted.

Vocational Models in Mental Health Rehabilitation

\section{Traditional Approaches to Work in Mental Health}

The notion that work contributes to physical and psychological well being is an ancient one that dates back to Greek physicians, Galen and Hippocrates (Pozner, $\mathrm{Ng}$, Hammond \& Shepherd, 1996). Accordingly, a strong tradition of using work as therapy has prevailed within psychiatric hospitals for over five hundred years (Ezersky, 1991; McCurrin, 1994; Schneider, 1998b; Vostanis, 1990). Since the 1960s, an increasing array of vocational rehabilitation models and programmes has accompanied people as they move from the psychiatric hospitals into the community (Garkse, 1999; McGurrin, 1994). Following deinstitutionalisation, which first commenced in Italy in the late 1960s, the initial focus was on 'train and place' models. These approaches included: sheltered workshops; training in work adjustment skills; job clubs and transitional employment (McCrum, Burnside \& Duffy, 1997; McGurrin, 1994; Schneider, 1998b). Critics argue that the 'train and place' programmes produce disability and dependence because of the notion that workers can remain indefinitely in these segregated work settings (Anthony, Cohen, Farkas \& Cagne, 2002; Bond, Drake, Mueser \& Becker, 1997). On the other hand, proponents argue that clients with severe impairments are not capable of working outside of protective settings and will deteriorate if not provided with this sheltered work environment (Bedell, Draving, Parrish, Gervey \& Guastadisegni, 1998; Drake et al., 1998). Dramatic differences, however, between sheltered and open employment environments in both requirements and opportunities, suggest that caution should be used when making generalisations between the two setting types (Mueller \& Wilgosh, 1991; Schneider, 1998). The supported employment approach, and the more recent innovations which have led to hybrid supported employment methods, are explored in the following paragraphs. 


\section{Supported Employment Approach}

Supported employment, a North American concept developed in 1986 by Paul Wehman, promoted the premise of forgoing the stepwise prevocational 'train and place' approach in favour of direct placement with on-the-job follow up. During the last decade, this concept has received increasing support from researchers (Becker, Drake \& Naughton, 2005; Bedell et al., 1998; Bond et al., 1997; Bond, 1998; Bond, 2004; Drake et al., 1994; Lehman, 1995; Schneider, 1998b). The supported employment outcomes used most often to measure vocational success are: securing employment; the length of time taken to secure a job; job tenure and number of hours worked (Drake et al., 1998). In a study that examined regional differences between ten supported employment agencies, Drake et al. (1998) found there were major differences in how the mental health centres addressed vocational goals. Job placement rates varied from 13 percent to 35 percent and programmes that achieved high employment outcomes, as defined previously in this paragraph, were found to pursue a variety of vocational approaches. These methods all emphasised close collaboration between clinicians, employment specialists and clients to achieve the goal of competitive employment. Programmes with low employment outcomes, continued to emphasise prevocational activities, such as day treatment and sheltered employment (Bond et al., 1997; Drake et al., 1998). In the twenty years since the inception of supported employment, concern about relatively low rates in both securing and keeping employment has led some providers to make dynamic adaptations. These hybrid approaches are described in the following paragraphs.

\section{Supported Employment: Hybrid Approaches}

In practise, many programmes, such as Boston University's 'Choose Get Keep' model, use hybrid approaches to supported employment in which skills training, job clubs and career planning are incorporated into vocational programmes. (Bond, et al., 1997; Danley, Sciarappa, McDonald-Wilson, 1992; Farkas, 1996). To increase the efficacy of these interventions, the researchers emphasise the use of methods that promote learning through active participation, including group workshops, goal setting and role-playing (Becker \& Drake, 1994; Becker et al., 1996; Diamond, 1998; Reddon, McNeil, Schmitke \& Trelenberg, 1992). The promoters of these hybrid approaches argue that, at the point of entry to the 
workforce, vocational training must continue to focus on increasing the number of critical job related skills that the individual has. This is because skills directly related to quantity and quality of work performance are essential to job success (Anthony, et al., 2002; Eikelmann \& Reker, 1993; Mueller \& Wilgosh, 1990). These elements, however, are not a formal part of the USA Federal supported employment guidelines, nor are they consistent with Wehman's 'place and train' conceptualisation (Bond et al., 1997). In addition, concerns about the significant mismatch between people with mental illness desiring to work, and those who are actually in work at any point in time, have led to a further shift away from Wehman's purist supported employment approach. The following paragraphs describe multidimensional models that combine supported employment with other approaches, such as transitional businesses.

\section{Multidimensional Approach to Vocational Opportunities}

There is much evidence to support the notion that there is no one best model for provision of vocational services (Farkas, 1996; McGurrin, 1994; Reker; Hornung; Schonauer \& Eikelmann, 2000; Wallace, 1993). Given the difficulties of helping people with mental illness integrate into the workplace, an array of vocational opportunities provides for a range of consumer needs, at varying points of their career development. For example, this array may include employment options such as transitional employment; volunteer work; clientbased industries (such as co-operatives); job clubs, as well as flexible employment options such as job sharing and supported employment (Bybee, Mowbray \& McCrohan, 1996; Chandler, Meisel, Hu, McGowen \& Madison, 1997; Dorio; 2004; Farkas, 1996; McCrum et al., 1997).

'The Village' programme situated in Long Beach, California, is an integrated service agency (ISA) model. It is a unique programme in that it focuses on both competitive and transitional employment. Chandler et al. (1997) conducted a three year independent study that found that 'The Village' achieved a job placement rate in competitive employment of 32 percent, with this rate increasing to 73 percent when transitional employment positions were included (Bond et al., 1997). These employment outcomes compare favourably with many of the dedicated supported employment agencies, particularly as 'The Village' is 
in a unique situation where client interest in work is not an eligibility requirement.

The literature also raises the question of whether a supported employment approach with pure emphasis on "place and train" in competitive employment, is more effective than multidimensional models that include options such as job clubs and transitional employment (Bybee, et al., 1996; Bond, 2004; Chandler et al., 1997; Chandler, Levin \& Barry, 1999; McGurrin, 1994; Farkas, 1996; Wallace, 1993). Thus, some of the literature that has been generated over the last decade describes a variety of flexible vocational interventions and techniques. This multidimensional approach is reflective of the diversity in each individual's capabilities and desire to work that often remains hidden by common diagnosis and general underemployment (Bybee et al., 1996; Bond, 2004; Chandler et al., 1997; Chandler et al., 1999; Krupa, McCourty, Bonner, Von Briesen \& Scott, 2000; Krupa, 2004)

\section{Vocational Rehabilitation Models: The International Experience}

Vocational rehabilitation exists in both a cultural and political context. Since the 1960s, North America has led the world in the practise of vocational integration within the competitive work environment, providing most of the evaluative research on employment issues in the mental health sector (Bond et al., 1997). This movement has also been supported by the fact that in the USA, since the early 1970s, employment and recidivism had been used almost exclusively as outcome measures in early psychiatric rehabilitation outcome studies (Anthony et al., 1992). While the literature from the USA has primarily focused on supported employment within the competitive work environment, a number of other countries have developed entrepreneurial economic enterprises to provide work opportunities for people with mental illness. 'Affirmative Businesses' are a popular model in Canada. These are consumer-driven enterprises that operate in partnership with both business representatives and service providers. They are usually subsidised in some areas, with key staff in facilitative and managerial roles, (Krupa et al., 2000; Strong, 1998). 
The European literature describes 'Social Firms'; a model established in Italy during the late 1960s when they were designed to replace the old psychiatric institutions. These firms are funded by local government and they aim at free market enterprise in order to provide jobs for people with disabilities, forming the cornerstone of community services. The social firms are underpinned by intensive job supervision, a non profit orientation and flexible, individualised workplace accommodations when required (Reker et al., 2000; Schneider, 1998b; Vostanis, 1990). The historical tradition of kin-based work niches, combined with the socialist influence that prevailed in post World War II Europe, has seen social firms and worker co-operatives become an extension of the cultural values that promote vocational opportunities for people with mental illness in Germany, Spain, Portugal, France and Scandinavia (Eiklemann \& Reker, 1993; Reker et al., 2000; Schneider, 1998b).

Developments in the United Kingdom were slower and more uneven (Mitchell, Betts \& Epling, 2002). Following the piloting of a small number of supported employment projects in the mid-nineties, the increased recognition of the value of such programmes has seen this approach become more widely adopted (Schneider, 1998b; Pozner et al., 1996). An increasing number of work projects now operate within the framework of small business enterprise, offering goods and services to the community. While most of these businesses offer unwaged long-term work as a stepping-stone to paid employment, a small number of social firms also provide paid employment (Midgley, 1990; Schneider, 1998b; Pozner et al., 1996). The British experience of employment opportunities for people with mental illness mirrors New Zealand's situation, which is explored under a separate heading (see p. 30) later in this chapter.

\section{Meaningful Employment for People with Psychiatric Disabilities}

While each person's experience of meaningful work is personal and uniquely influenced by the interplay of many variables, people with mental health disabilities share a common theme in the experience of recovering a more positive sense of self, beyond the limits of the disability (Strong, 1998; Krupa, 2004). Moving beyond the constraints of the prescriptive practises that are 
consigned to specific vocational models, the literature has been examined to discover the elements that cause work to be meaningful to people with psychiatric disabilities.

\section{Meaningful Work: Relationship to Recovery}

From the perspectives of Deegan (1992) and Lette (1992), who are well-known writers and consumer advocates, meaningful employment is believed to be essential to the recovery process. Deegan (1992) explores this notion further, stating that the process of participation in meaningful work appears to interrupt the cycle of disempowerment and despair. Work is seen to be meaningful when it fits with the person's beliefs, interests, goals and sense of self. Therefore, meaningful tasks and activities not only engage a person's time and energy, but they also engage the person by forming a connecting bond (Granerud \& Severinsson, 2006; Strong, 1999). Employment has been identified as a strong predictor of successful community adjustment for people with psychiatric disabilities. As the person shifts in consciousness from 'illness' to 'work' issues, the recovery process is supported and enhanced (Gioia, 2005; Krupa, 2004; Marrone \& Golowka, 1999; Mueller \& Wilgosh, 1991; Sundar \& Ochocka, 2004).

Mental health researchers have found that being in work is positively associated with a general sense of well-being, which appears to protect the worker against the return of symptoms (Becker, Drake \& Naughton, 2005; Becker \& Drake, 1994; Blankertz \& Robinson, 1996; Reddon et al., 1992; Schneider, 1998b). Corroborating evidence is also derived from studies which have determined that people discharged from psychiatric hospitals who have a job, are much less likely to be re-hospitalised than those who are unemployed, regardless of pathology (Reker, et al., 2000; Schneider, 1998b, Tsang, 2000). There is also a significant decline in use of health services generally, when these individuals are engaged in work (Blanketz \& Robinson, 1996; Rogers, Anthony, Cohen \& Davis, 1997; Schneider, 1998b; Sundar \& Ochocka, 2004).

In summary, people with mental illness who are employed fare better, although it is not clear if work leads to an improvement in aptitude, or if people who possess 
higher skill levels are more able to retain their employment (Warner \& Polak, 1995). The research evidence concurs, however, that ongoing work activity has demonstrated clinical benefits and significant positive effects on perceived selfcompetence and independence (Blankertz \& Robinson, 1996; Bond, 2004; Reddon, et al., 1992; Reker, et al., 2000; Schmitke \& Trelenberg, 1992; Schneider, 1998b; Tsang, 2000).

\section{Meaningful Work: Relationships to Other Life Domains}

The belief that the self-esteem of adults with psychiatric disabilities is linked to their employment status has not received strong and consistent research support. Torrey, Mueser, McHugo and Drake (2000) found an inconsistent link between work and self-esteem, in that self-esteem does not appear to be a predictor of future work achievements. Self-esteem appears to be a relatively stable trait that is reflective of general life satisfaction and affective symptoms, rather than dependent on functional status (Torrey et al., 2000). In a study conducted by Day and Alon (1993), the strongest contributor to general life satisfaction was found to be leisure. Work was found to be a major contributor, however, as it allowed the participants to engage in activities that contribute more directly to overall life satisfaction. For people with psychiatric disabilities, social networks have been found to be important to the process of obtaining and maintaining employment (Danley et al., 1992; Torrey et al., 1998; Wallace, 1993). Some studies, however, have identified that work can sometimes increase a person's sense of isolation because of their reduced access to their former social networks, which they have not been able to replicate at work (Becker et al., 1997; Bedell et al., 1998; Strong, 1998). This research demonstrates how vital it is that work is interconnected with other roles and activities, as successful employment does not guarantee satisfaction or fulfilment in other life domains (Marrone \& Golowka, 1999).

\section{Meaningful Work: Relationship to Job Selection}

Despite the rhetoric within the literature about job matching and job preferences, most supported employment job placements are into unskilled, entry-level jobs (Baron \& Granger, 1997; Krupa, 2004; Michon et al., 2004). Bond et al. (1997) 
produced the first synthesis of empirical findings on supported employment for people with psychiatric disabilities. Entry level jobs such as food services or cleaning are the most popular type of placement, with the percentage of supported employment clients who work in such positions ranging from 35 percent to 62 percent. The range of clerical placements was between 6 percent and 19 percent, with only 10 percent to 20 percent of placements being into skilled positions. Bond et al. (1997) found that there was no evidence to suggest many clients move on from these entry-level positions. Studies have demonstrated, however, that job tenure was twice as long for people who had got a job within a preferred area, than people who had got jobs that did not align with their preferences (Bond et al., 1997; Bond; 2004; Gevey \& Kowal, 1995). Also it has been demonstrated that the large majority of people who signed up for supported employment had realistic, explicit job preferences (Eikelmann \& Reker, 1993). Marrone and Golowka (1999) argue, however, that while it is important to assist people to dream and think about careers, they also need to develop an understanding of an essential fact of work life, a person cannot have a career without a job.

\section{Meaningful Work: Relationship to Job Tenure}

Within recent mental health vocational literature, authors have expressed concern that service users and providers continue to be focused on entry to work, with little attention to job retention issues (Becker et al., 2005; Gioia, 2005; Krupa, 2004; Lapsley, 2003). During a study that focused on job termination, Becker et al. (1997) found that the majority of workers with mental illness had experienced an unsatisfactory end to their employment. Within the population under study, 40 percent had been dismissed and 14 percent had resigned without having other positions. According to Becker et al. (1998) these unsatisfactory job terminations were associated with difficulties that arose on-the-job, in a range of areas. These aspects included interpersonal functioning; mental illness; dissatisfaction with the job; quality of work; medical illness; dependability and substance abuse. Clients with better work histories, however, were found to be less likely to experience an unsatisfactory job termination (Becker et al., 1998; Mueller \& Wilgosh, 1991; Tsang, 2000). The multidimensional nature of the issues related 
to job retention underscores the notion that both clinical and vocational expertise is required in the workers support system (McHugo, Drake \& Becker, 1998; Mueller \& Wilgosh, 1991).

A three-year study involving 'The Village' model found that this programme had achieved statistically impressive employment outcomes. Only 12.9 percent of people engaged in this programme, however, retained their open employment positions for more than one-year (Chandler et al., 1997). Further studies into job tenure in dedicated supported employment agencies, found that after six months, job retention rates were reported to be between 35 percent and 59 percent (Bond et al. 1997; McGurrin, 1994; Wallace 1993; Wehman et al., 1991). Research has also demonstrated that people placed from the traditional training and placement models, demonstrated similar job retention rates to those people in supported employment programmes, although the rate of securing positions was significantly higher in the latter approach (Bedell et al., 1998; Wallace, Tauber \& Wilde, 1999).

There is a general belief that workers with psychiatric disabilities' on-the-job support needs, fade over time, in a simple linear fashion. Wallace (1993) and McHugo et al. (1998), however, argue that a lifelong succession of fadings is a better way to conceptualise the work support needs of people with psychiatric disabilities. In this regard, Becker et al. (1998) found that workers with psychiatric disabilities need a variety of ongoing supports to address problems that arise on the job, even when they are initially satisfied with the job and the work environment. They particularly emphasise the client's need for assistance in the application of social skills to master the challenges of the work culture and its interpersonal requirements. Researchers have demonstrated a worker who is a poor producer, but is socially adept might survive on the job longer than one who is weak in both areas (Becker et al., 1998; Dorio, 2004; Mueller \& Wilgosh, 1991; Tsang, 2000). Some evidence suggests that a structured skills training module, which focuses on these worker concerns after job placement rather than prior to job placement, promotes job tenure. In contrast, job retention was not found to be related to pre employment job skills training (McCrum et al., 1997; Wallace et al., 1999). In summary, job retention rates across all the vocational 
models during the twenty years since the inception of the supported employment approach, clearly demonstrate the need for further investigation and intervention (Becker et al., 1998; Bozzer, Samson \& Anson, 1999; Dorio, 2004; Michon et al., 2004; Mental Health Commission, 1999).

\section{Meaningful Work: Relationship with Disclosure}

Within the employment context, the issues surrounding an individual's disclosure about their history of mental illness and prior mental illness-related offending are inherently complex (Auerbach \& Richardson, 2005; Granger, 2000; Krupa, 2004; Mental Health Commission, 2006; Schneider, 1998a). Both nationally and internationally, people who self disclose about their mental illness are usually very concerned about what it may do to their reputations and their careers (Granger, 2000; Krupa, 2004; Mental Health Commission, 2006; Schneider, 1998a). Although the depth of this complexity is acknowledged in some research articles, there is scant reference to disclosure within the body of the mental health vocational literature (Killeen \& O’Day, 2004; Krupa, 2004). In addition, the literature reviewed for this study omitted any reference to managing the additional burden of illness-related offending.

Krupa (2004) suggests taking a cautious approach to disclosure, thereby preventing issues of stigma and discrimination negatively impacting on the individual's work prospects. In situations where job accommodations are desired, however, or when people are concerned that their symptoms of illness may affect their job performance, Granger (2000) and Schneider (1998a) strongly recommend that workers disclose to at least one manager. Conversely, the literature suggests a cautious approach to disclosing to colleagues, who unlike managers, are not bound by confidentiality protocols (Granger, 2000; Krupa, 2004; Schneider, 1998a). In contrast to Krupa's (2004) more cautious approach to disclosure, there was a consensus within the rest of the literature that managers generally deal well with the information. In addition, these researchers found that people reported feeling strengthened by the knowledge that their disclosure was positively received by their manager (Granger, 2000; Mental Health Commission, 2006; Schneider, 1998a). Hence, Granger (2000) and Schneider (1998a) concur that while some people were adamant about not disclosing, most 
people who had the support of a vocational practitioner disclosed their issues to managers with few difficulties.

Work as a Dynamic Component of Mental Health Rehabilitation Practise Work should not be viewed as an independent factor in rehabilitation, but as a dynamic component of the rehabilitation process in which practitioners apply their clinical insights and endeavours to the vocational development of their clients (Anthony et al., 2002; Blankertz \& Robinson, 1996; Bond, 2004; Bybee et al., 1996; Garske, 1999; Krupa, 2004; McGurrin, 1994; Vostanis, 1990). Traditionally, mental health clinicians have regarded work as a potential stressor to be avoided by their clients, thereby averting relapse and declining function. (Baron \& Granger, 1997; Bond, 2004; Gioia, 2005; Killen \& O’Day, 2004; Krupa, 2004; Marrone \& Golowska, 1999; Pratt, Gill, Barrett \& Roberts, 1998). It has been demonstrated, however, that clinicians make dramatic attitudinal shifts when they become immersed in integrated vocational programmes (Becker \& Drake, 1994).

Within the literature reviewed for this study, researchers express concern about low rates of job retention, and wide variances between programmes, in relation to their vocational outcomes. In addition, there is a mismatch between people desiring to work (66\% to $70 \%)$ and those consumers who are actually in work at any point in time (between $8 \%$ and $15 \%$ ). This situation leads researchers to state there is an urgent need to develop key concepts and dimensions related to the area of employment in mental health rehabilitation (Anthony et al., 2002; Blankertz \& Robertson, 1996; Bond, 2004; Gioia, 2005; King et al., 2006; McCrum, et al., 1997; Pratt et al., 1998; Reker et al., 2000; Schneider, 1998b; Van Dongen, 1996). Historically, incentives were given to programmes to enhance vocational outcomes without identifying any specific vocational practise guidelines. Therefore, it is not surprising that researchers have found that vocational programmes may not be underpinned by a solid knowledge base (Drake et al., 1998; Schneider, 1998b). 
Torrey et al. (1998) conducted a research study that identified nine practice guidelines for clinicians working in the area of vocational rehabilitation. The guidelines emphasise vocational exploration and job matching; interconnections with social networks; clinical and rehabilitation planning; support and problem solving methods; and self management techniques that focus on the areas of substance abuse, wellness and on-the-job interpersonal issues. The intent of these guidelines is also echoed in Danley et al.'s (1992) overview of the 'Choose-GetKeep' model, which emphasises: person-centred goals; a client environment match; prescriptive assessment, planning and intervention, and the development of skills and supports required to promote satisfaction and success within the selected work setting. More recent studies have emphasised the importance of establishing comprehensive, integrated vocational pathways. This process involves developing a positive vocational identity, which is distinct from, but accommodates features of the worker's illness, and then incorporates the required workplace supports (Gioia, 2005; Killeen \& O’Day, 2004; Krupa, 2004).

After completing an extensive review of the vocational rehabilitation literature produced since the mid 1980's, Tsang, (2000) states that there is ongoing conflict within the research findings about the ability of psychiatric symptomatology and diagnosis to predict employment outcomes. Tsang (2000) concludes premorbid functioning, work history and social skills are more positive indicators of successful work outcomes. In addition, Anthony (1994) and O'Neill and Bertollo (1998) argue that the prevailing focus on demographic and clinical variables is not as useful to the vocational programmes or their service users, as the information gathered from research into work readiness factors. These elements are: personal preference; satisfaction with the current situation; commitment to change; self efficacy; environmental factors and self awareness. Unlike many demographic and clinical factors, these readiness variables can be changed through various kinds of vocational interventions, and thus Anthony (1994) concludes that they are perhaps a more meaningful target for research and to define practise guidelines. 
Vocational Rehabilitation: The Forensic Psychiatry Perspective

\section{Forensic Community Rehabilitation: A Vocational Perspective}

In forensic psychiatry, rehabilitation practitioners are required to consider the needs and aspirations of their clients, within the legal interface between the criminal justice system and mental health legislation. Hence, a key factor facing staff working in forensic psychiatry is the challenge of reconciling the tensions between a rehabilitative and risk management approach (Couldrick \& Aldred, 2003). These contradictions and challenges apply at all levels of secure care, however, they are particularly brought into question when clients have moved back into the community. With this shift, forensic and vocational rehabilitation practitioners have to navigate through the frequently contradictory goals of social inclusion, treatment and public safety (Couldrick \& Aldred, 2003; Joe, 2003).

People returning to the community from a secure treatment setting require a higher level of follow up support than many other mental health client groups. Swartz et al. (1998) state that effective community treatment for individuals who have a forensic history requires careful attention to medication adherence, within the context of readily available, integrated substance abuse and mental health treatment services. In addition, forensic psychiatric services recognise that care plans for those people who have been discharged into the community, will be directed by the risk, and by the public perception of risk, that their service users pose to the community (Joe, 2003). When considering the choice of work placement, the practitioner has to balance public safety with the client's work aspirations and rights to equal opportunities (Joe, 2003). Hence, the job seeking agreement between the individual and vocational practitioner would exclude jobs that may increase the risk factors, for example easy access to drugs and alcohol (Becker et al., 2005).

People who have been through forensic services have a two pronged experience of discrimination. In addition to having a label of psychiatric illness, they also have to contend with the implications of being under the care of forensic psychiatric services (Joe, 2003). For many employers, this dual label signals that the prospective employee has the potential to be both unpredictable and 
dangerous. Such stigmatisation often impedes these individuals' access to work opportunities. People with a forensic history may have to disclose offences and account for long periods in secure facilities. A significant risk of disclosure is that it may lead to rejection by employers and colleagues solely based on the prospective employee's psychiatric and offending history, rather than being assessed according to their work performance (Joe, 2003; Krupa, 2004).

The principles of social inclusion recognise that people excluded or impeded from the decision making rights and the social opportunities that affect their lives are denied citizenship (Freire, 1972; Joe, 2003). Many mental health clients are excluded from citizenship rights due to the stigma of mental illness and prior illness-related offending; devastating life events which are compounded by the resultant interruptions to education and employment (Joe, 2003; Taylor \& Gunn, 1999). Work is an activity that promotes social inclusion (Freire, 1972; Mitchell, et al., 2002). Hence, the social inclusion of individuals with mental illness into work, may possibly ameliorate the damaging effects of social exclusion and previous victimisation, and thereby reduce their risk of re-offending (Mitchell et al., 2002).

\section{Rehabilitation and Work in Forensic Psychiatry: The Literature}

The assessment of risk in relation to the probability of people with a forensic history re-offending continues to be the subject of extensive research (Lindqvist $\&$ Skipworth, 2000). In contrast, authors writing from a rehabilitation perspective concur that there is scant reference to rehabilitation within the forensic literature (Couldrick \& Aldred, 2003; Lindqvist \& Skipworth, 2000; Lloyd, 1995; Tracey, 2000). In addition, authors note from the rehabilitation, vocational and occupational therapy perspectives, there is a paucity of research in the area of forensic psychiatry (Lloyd, 1995; Couldrick \& Aldred, 2003). Lloyd (1995) states from a practise standpoint, that rehabilitation programmes offered by occupational therapists in forensic psychiatry have mostly placed importance of vocational rehabilitation, and work related programmes to develop work habits and skills. In Couldrick and Aldred's (2003) text book on Forensic Occupational Therapy, however, the authors state that by the time they noticed that they had included little on work or vocational rehabilitation; it was too late to rectify. With 
increasing professional requirements for practise to be validated by evidence, the deficit of vocational and rehabilitation literature in relation to actual practise within forensic settings is of concern. In addition, the absence of qualitative studies illustrating the forensic service users' lived experience means their unique perceptions of what constitutes meaningful rehabilitation, and their notions about which interventions support their community readjustment and continued recovery, remains unexplored.

\section{Risk Management: Vocational Rehabilitation Perspective}

While risk identification is widely accepted as being central to treatment and assessment routines within secure care hospitals, the understanding of violent behaviour in individuals with psychiatric disabilities becomes even more crucial post discharge (Couldrick \& Aldred, 2003; Joe, 2003). Accordingly, the community reintegration process is heavily influenced by a risk minimisation approach. Vocational rehabilitation practitioners, however, also have a role in trying to change attitudes within the employment sector in order to develop social inclusion and to challenge discriminatory practises (Sundar \& Ochocka, 2004). Therefore, understanding the predisposing factors to violence strengthens the practitioner's advocacy and educative roles (Couldrick \& Aldred, 2003).

Largely fuelled by the move towards deinstitutionalisation which commenced in New Zealand in the early nineties, the relationship between mental illness and violence has been the subject of considerable debate, both within the mental health literature and the media (Simpson, McKenna, Moskowitz, Skipworth \& Barry-Walsh, 2003; Taylor \& Gunn, 1999). From a historical and cultural perspective, Monahan (1992) purports throughout time, most societies have drawn a relationship between mental illness and violence. Studies have shown, however, that the vast majority of people with psychiatric disabilities do not pose an increase risk of danger to others (Taylor \& Gunn, 1999). Swartz et al. (1998) elaborate on these findings further, stating that there is an absence of a solid research base which directly links psychiatric clinical and symptom variables with an increased risk for violent behaviour. 
Some groups of people with psychiatric disabilities, however, are at a statistically higher risk of being more violent than the general public, but the size of the risk is considered to be minimal (Taylor \& Gunn, 1999). In a further analysis of this finding, Swartz et al. (1998) conclude that the increase and magnitude of risk for violence was represented by two key variables, substance abuse and medication non-compliance. These researchers also suggest that a third variable, poor insight into illness, may potentially lead to both substance abuse and non compliance with medication. Consequently, this combination may increase the risk of violence. Independently, however, insight into illness and non compliance did not significantly increase the risk for violence.

There is consensus within the literature reviewed for this study that people with co-occurring mental health and substance abuse problems are at greater risk of violent behaviour towards others (Swanson, Borum, Swartz \& Hiday, 1999; Swartz et al., 1998; Taylor \& Gunn, 1999). Taylor and Gunn (1999) also highlight the considerable co-morbidity between personality disorders and substance abuse. Consequently, they found that people whose diagnostic profile includes mental illness, personality disorder and substance abuse, comprise the majority within the broader population of people with psychiatric disabilities who are violent to others (Taylor \& Gunn, 1999). Swanson et al. (1999) also add the lack of contact with speciality community mental health services to the combination of predisposing factors. The research evidence concurs that the effects of substance abuse and high risk environmental influences, such as victimisation described in the following paragraph, combined with the symptoms of psychiatric illness were considered to increase the risk of violent acts towards others (Swanson, et al., 1999; Swartz, et al., 1998; Taylor \& Gunn, 1999).

\section{Forensic Psychiatry: A Cultural Perspective}

Studies completed in both Sweden and USA, found that the cultural groups who are significantly over-represented within forensic psychiatry statistics, belong to communities who have been exposed to disproportionately high levels of socioenvironmental trauma (Kristiansson, Sumelius \& Sondergaard, 2004; Swartz, et al., 1998). Both research groups found significantly higher rates of an individual or family experience of victimisation within the immigrant and African 
American populations, who are significantly over-represented within Swedish and American forensic psychiatry services respectively. The combination between being a member of one of these over represented populations, and a victim of crime and/or violence, was the only predictor of violence within the mental health population that emerged as statistically significant (Swartz et al, 1998). Hence, cultural identity is only of interest as a social designation, in that it may correlate with environmental precipitants to the experience of victimisation. Therefore, both Swedish and North American research groups concur that the apparent cultural effect within forensic psychiatry is explained by socioenvironmental trauma (Kristiansson et al., 2004; Swartz, et al., 1998).

Within the New Zealand context, prior to 1970, Māori had been underrepresented in psychiatric hospitals, a trend that has significantly reversed over the last thirty five years. Now the statistical evidence is that Māori admissions to inpatient facilities have become two to three times higher than for non-Māori (Durie, 1998). Chaplow, Chaplow and Maniapoto (1993) also highlight this from the standpoint of both general and forensic psychiatry, stating that Māori and Pacific Island people are over-represented in New Zealand mental health institutions. Durie (1998) describes the reasons behind this statistical trend as being diverse and complex. He states these explanations range from more accurate statistical gathering and utilisation of psychiatric facilities on one hand, to urbanisation, cultural bias and frank discrimination at the other end of the spectrum. Therefore, mental health service providers, planners and researchers need to be mindful of these factors, particularly in terms of the historically constituted positions related to cultural oppression and the consequential impact on psychiatric hospitalisation and unemployment statistics (Chaplow et al., 1993; Durie, 1998; Jungersen, 2002).

\section{Work Issues in Forensic Psychiatric Rehabilitation: A Summary}

After a mean stay of four years, four months (Simpson, Jones, Evans \& McKenna, 2006), people who are discharged from secure treatment facilities in New Zealand are often work-ready. Assertive forensic community follow-up ensures these clients are well stabilised on their medication regimes, and continue to abstain from any preadmission substance abuse. In addition, newly 
discharged clients' readiness-for-work is increasingly supported by the shift towards inpatient rehabilitation programmes (Lloyd, 1995). This rehabilitative approach is underpinned by practitioner and service acknowledgment of the importance of work and social dimensions in restoring and promoting mental health gains (Couldrick \& Aldred, 2003; Lindqvist \& Skipworth, 2000; Lloyd, 1995; Tracey, 2000). Therefore, this rehabilitative approach co-exists alongside the more traditional interventions of medication; risk assessment and basic support (Couldrick \& Aldred, 2003). That forensic psychiatry populations compare favourably with matched criminal populations with respect to recidivism is not surprising, given the vastly disparate nature of treatment and rehabilitation (Couldrick \& Aldred, 2003). Therefore, those individuals who are referred to specialist mental health vocational services are frequently ready and willing prospective employees.

\section{Review of the New Zealand Literature: Mental Health and Work}

Within New Zealand's mental health strategic plan, operationalised over the last nine years within two guiding documents, there is little reference to employment issues. The Ministry of Health's (1997) 'National Mental Health Standards' briefly states that mental health services should provide consumers' access to an appropriate range of education, training, work and employment opportunities. The second guiding document, published by the Mental Health Commission (1998), is the 'National Blueprint for Mental Health Services in New Zealand'. This document provides the strategic plan until 2010, detailing mental health service developments required to implement the National mental health strategy for "more and better mental health services". Within this document, employment is again only mentioned very briefly. The Blueprint recommends ten full time equivalent vocational staff per 100,000 total population to achieve the primary goal of long-term paid employment. This recommendation is further qualified by stating that employment support is not always seen as a health responsibility, with other Government agencies, including Work and Income New Zealand (WINZ), also having a role. The last brief reference to work is that specific strategies are needed to address education and employment issues for Māori, including through access to kaupapa Māori services. 
A Māori kaupapa ${ }^{6}$ perspective enables work issues to be addressed within a culturally appropriate context, as work has different meanings within different cultures. Māori culture has its roots in a traditional kin based society, whose values of collective ownership and shared productivity are at odds with the prevailing concept of work within many dominant western cultures. The Royal Commission on Social Policy (1988) states in their discussion booklet on work:

\begin{abstract}
Work has different meaning and significance in different cultures. In a number of societies work measures the status of people and contributes to a sense of identity. For Māori and Pacific Island peoples however, work is less important than kinship in determining the position of a person within a community. (p. 4)
\end{abstract}

As discussed previously under the heading Forensic Psychiatry: A Cultural Perspective (see p. 28) Māori are significantly over-represented in the population under study. Much of the health gap experienced by Māori can be attributed to poorer socio-economic status (Health Funding Authority, 2000; National Health Committee, 1998). In making further linkages between health and socioeconomic status, in June 2006, unemployment rates for Māori were 8.5 percent, more than twice the national rate and over three times the rate for Pakeha (Department of Labour, 2006). While unemployment rates for Māori have declined dramatically since 1999 when they exceeded 25 percent (Macaskill, 2000), they continue to replicate the pattern of being twice the national average. The three core constructs which underpin Te Tiriti o Waitangi ${ }^{7}$; protection; partnership and participation, were accepted by the New Zealand Department of Health in 1990 as determinants of health provision. Soon after this Department of Health mandate, Chaplow, et al. (1993) reported that efforts were being made in New Zealand to address cultural differences within health institutions. Increasingly Māori values of wairuatanga, manaakitanga and whanaungatanga (spirituality, caring and family ties) are being regarded as core elements of mental health treatment plans for Māori.

The first New Zealand National Employment Forum (1999) was a collaborative venture between service users and providers of disability services. The forum's

\footnotetext{
6 'Kaupapa' is a Māori word for foundation, basic ideas and rules (Ryan, 1993).

7 'Te Tiriti o Waitangi' is the Māori phrase for the Treaty of Waitangi (Durie, 1998)
} 
published conclusions state that the employment capabilities of mental health consumers have been generally underestimated. Mainstream disability and mental health employment services were described as being generally ineffective for helping people find work. This was attributed to the employment specialists having low vocational expectations of people with mental illness, with their practise focusing on prevocational support and training, or segregated work options. Another major contributing factor was stated as being an insufficient number of employment services to meet needs. In addition, the majority of service providers were perceived as having inadequate knowledge and skills, in both mental health issues and employment issues.

The report generated by the New Zealand National Employment Forum (1999) also reviewed the current socio-political issues that governed New Zealand's labour market. One of these influences is the international trend to casualisation ${ }^{8}$ of workplace conditions which has been seen to have a significant impact on people with disabilities through unemployment, underemployment and competition for jobs (Ansley, 1999). Many people with psychiatric disabilities have been adversely affected by these changes because they tend to lack training, consistent work experience and personal contacts in the labour market. They may also face discrimination from employers and work colleagues. These concerns were also highlighted in the popular press, when the Listener printed an article on supported employment for people with mental illness.

\section{If mental health consumers are to become totally integrated into the workforce, it will require not only a concerted effort on the part of mental health services and consumers themselves, but also a significant change in attitudes from mainstream employers. (Spence, 2004, p. 28)}

In an attempt to address the employment issues raised within both the disability sector and the media, the New Zealand Disability Strategy (2001) was designed to be implemented as a Government directive (Minister for Disability Issues, 2001). The socio-political intent of this strategy is to have the employment,

\footnotetext{
${ }^{8}$ Casualisation of the workforce refers to the process whereby permanent full time and part time positions are replaced by a casual workforce who are employed on an 'as required' basis (Ansley, 1999).
} 
health and income support sectors working closely together to ensure their policies and operational practises are aligned to meet the employment needs and aspirations of people with disabilities. Reflecting New Zealand disability consumers' request for 'real jobs, for real pay', the emphasis in this innovative and dynamic strategy is on finding and sustaining open employment and strengthening people's natural supports to achieve this.

As a supplement to the New Zealand Disability Strategy, the Ministry for Social Development (MSD) (2001) also launched 'Pathways to social inclusion/ Nga ara whakauuru ki te whanui: Improving vocational direction for people with disabilities'. This document announced a new direction in vocational service provision for people with disabilities. In accordance with new social policy, the overall focus of this document is the shift in emphasis towards social development. This move is counterbalanced by a reduced emphasis on the social protection ethos that focuses on providing a safety net against hard times. In relation to unemployment, this MSD document aims to herald the development of new strategies to encourage people into work. These strategies include funding for employment assistance and employment incentives, and changes in work and income practises to encourage people to return to work and thereby reduce the number of people on Benefits.

In 2003 the New Zealand Mental Health Commission echoed the directions expressed in the New Zealand National Employment Forum (1999) and the subsequent Government led New Zealand Disability Strategy (2001) in an employment focused occasional paper (Lapsley, 2003). This document provides a synopsis of current trends and new developments in employment, from both a New Zealand mental health and a legislative perspective. The paper highlights the lack of attention being given to the problem area of job retention. The New Zealand Mental Health Commission is also due to publish a research study identifying the risks and rewards associated with disclosure in employment. Within a preview in the 'Like Minds, Like Mine' newsletter, the Mental Health Commission (2006) states this study is uniquely focused on how disclosure impacts on an individual's recovery. The Mental Health Commission (2006) found that people with mental illness are initially apprehensive about the impact 
of their disclosure. In general, however, it appears that supervisors and colleagues deal well with the information, and that the process strengthens the person who's made the disclosure.

This overview of employment issues for New Zealander's who are affected by mental illness relied on government publications; the popular press and conference publications. Many of these publications are again referenced in the following chapter, which compares the international evidence with the sociopolitical issues identified within the New Zealand literature.

The New Zealand and International Experience: A Socio-political View.

The literature reviewed for this study demonstrates that since the 1960s, both North America and Europe were able to have different, but equally proactive responses to meeting the vocational needs of people with psychiatric disabilities (see p. 16). A number of dedicated New Zealand vocational specialists have looked to overseas literature and models to provide innovative consumer enterprises and supported employment approaches (Harris, 1995; Warriner, 1993). The absence of vocational rehabilitation strategies within the New Zealand mental health operational plans, however, places vocational rehabilitation as a low priority, with minimal resources being allocated to this. Clearly, vocational rehabilitation does not exist in isolation; it must interact with the politically driven labour market as well as the wider community. North America, Britain and some European countries have very strong legislation protecting jobs (Midgely, 1990; Schneider, 1998b). In contrast, New Zealand has been less vigilant about job protection for people with disabilities (Lapsley, 2003; Mental Health Commission, 1999; New Zealand National Employment Forum, 1999). Until the New Zealand Disability Strategy (2001), there was no overarching, integrated government policy or funding mechanism, which spanned the labour market and the health, disability, employment and income support sectors, in order to address the employment needs of people with disabilities.

The New Zealand Disabled Persons Employment Promotion Act (1960) was repealed in February 2006. It continues to be the legislation which governs 
segregated employment opportunities for people with disabilities until June 2007. The repeal of this act was subject to much contentious debate within the disability sector, the media and health related literature (Klos, 2005; Stent, 2005). Critics of the repeal had concerns that the new workplace requirements will lead to closures of existing sheltered work sites. They perceive that this would eventually lead to job losses for workers with higher levels of disability and additional burdens of responsibilities for their families and whanau (Stent, 2005). Proponents of this legislative change support the intent behind this repeal which is outlined in the New Zealand Disability Strategy (Minister of Disability Issues, 2001). This is to create more work opportunities for people with disabilities within the open work force, with access to the employment conditions that conform to the Holidays Act (2003) and the Minimum Wage Act (1983).

Throughout the nineties, New Zealand had relatively high levels of unemployment compared with current low level of 3.6 percent (Department of Labour, 2006). This situation, combined with the lack of employment legislation for people with disabilities, has seen 'Affirmative Businesses' ${ }^{9}$ develop in tandem with supported employment programmes. While acknowledging that most people with mental illness have a primary desire for 'real work, for real pay', leaving job placement to market forces ignores the non-economic rewards of employment (Krupa et al., 2000; Schneider, 1998b). The European experience of 'Social Firms' and the research evidence from the 'Village Model', as described in preceding paragraphs in this literature review (see p. 15), have demonstrated the value of combining small transitional employment opportunities with supported employment (Chandler et al., 1997; Krupa et al., 2000; McCrum, 1997; Schneider, 1998b). These consumer driven enterprises offer alternatives to the traditional sheltered workshops. They are a viable response to service users' demands for better quality services with more participatory and democratised structures, offering a stepping-stone to open employment (Krupa et al., 2000).

\footnotetext{
${ }^{9}$ The affirmative business approach develops partnerships between consumers of mental health services, service providers, and business people. By facilitating the production of goods and services which can be sold in the broader community, it creates job opportunities for people with psychiatrics disabilities. (Krupa et al., 2000, p. 13)
} 
Very low levels of unemployment are now being enjoyed in New Zealand. At 3.6 percent in August 2006, New Zealand currently has the second lowest national unemployment rate in the Organisation for Economic Co-operation and Development (OECD) (Department of Labour, 2006). In 2003, however, despite a rapidly decreasing general unemployment rate since the late nineties, significantly more New Zealanders with disabilities were unemployed (an increase of 19.5 percent over five years), and complained about discrimination than in the mid 1990s (Mintrom \& True, 2004). This acknowledges people with disabilities to be one of the most disadvantaged groups in the current New Zealand employment environment (Mintrom \& True, 2004). The discriminatory practices of some employers and workers can create even more complex employment barriers for people with mental illness (Mental Health Commission, 1999). Unfortunately, nationally and internationally, these individuals have to overcome substantial barriers in seeking and securing employment (Auerbach \& Richardson, 2005; Bozzer, et al., 1999; Killen \& O’Day, 2004; King et al., 2006; Lapsley, 2003; Michon et al., 2004; Mental Health Commission, 1999).

The changing context of mental health care has been driven by the principles of community integration, a paradigm shift that has impacted, both positively and negatively, on the public perception of mental illness. Recent New Zealand research demonstrates that people with psychiatric disabilities pose a minute amount of risk to the general public, and their contribution has been falling over the years since deinstitutionalisation (Simpson et al., 2003). The New Zealand public, however, has been subjected to an opposing sensationalist view from the media (Spence, 2004; Smellie, 2006). By reporting uncritically, journalists have helped shape a negative societal view that has further marginalised people with mental illness within the employment environment.

The paucity of the literature from New Zealand means that it is difficult to accurately establish meaningful employment indicators from a mental health consumer perspective. However, the brief consumer statements from the inaugural New Zealand National Employment Forum (1999) are reflective of the consumer views expressed within the international literature. While New Zealand consumers agree with their North American counterparts, that working may 
mean the end of a lifestyle and result in the loss of supports and social contacts, they concur that their principal expressed desire is "for real jobs, for real pay" (New Zealand National Employment Forum, 1999, p. 7). From the wider sociopolitical perspective, the body of evidence within the international literature reviewed in this chapter aligns with the pressing political and sector directions outlined in the New Zealand Disability Strategy (2001) to: "Enable disabled people to work in the open labour market (in accordance with human rights principles) and maintain an adequate income" (Minister of Disability Issues, 2001, p.11). This strategy has helped to raise general awareness of disabilities, and has consequently also exposed the forces of hegemony and injustice within society:

Disability relates to the interaction between the person with the impairment and the environment. It has a lot to do with discrimination, and has a lot in common with other attitudes and behaviours such as racism and sexism that are not acceptable in our society. (Minister of Disability Issues, 2001, p. 3)

Vocational rehabilitation occurs at the interface between mental health services and the politically driven labour market, therefore the response from rehabilitation practitioners must be two pronged. The mental health sector needs to be responsible for ensuring that vocational exploration is integral to service users' treatment and support plans (Garske, 1999; Gioia, 2005). General underemployment of people with disabilities, however, essentially hinges on socio-political factors, with changes required at both a local and a national level (Killen \& O'Day, 2004). Since the New Zealand Disability Strategy (2001), there is a growing expectation from government that people who are work-able should be encouraged to participate in open employment. Improving the employment chances of people with psychiatric disabilities, however, will require a mix of proactive interventions at the individual and political levels (Krupa et al., 2000; Schneider, 1998b; Warriner, 1993).

\section{Summary}

Since the mid 1980s, North America has led the world in vocational rehabilitation innovations, which have promoted workplace and community 
integration for people with psychiatric disabilities. In contrast, within New Zealand's mental health strategic plan, operationalised within two guiding documents, there is scant reference to employment issues (Mental Health Commission, 1998; Ministry of Health: Manatu Houora, 1997). Both in New Zealand and internationally, people with psychiatric disabilities identify financial resources and employment as the areas that still present the greatest barriers to community integration (Mallik, Reeves \& Dellario, 1998; Mental Health Commission, 1999). Anthony et al. (2002) concluded that a prejudiced society is a significant barrier to people with psychiatric disabilities; therefore it is not an inaccessible environment that impedes these individuals from achieving employment, but inaccessible people. When these individuals have also been engaged with forensic psychiatric services, they have even less access to the existing range of work and social opportunities on offer, making them one of the most excluded groups within the community (Joe, 2003).

The international literature reviewed for this thesis provides both directions and insights to guide vocational rehabilitation practise, and has identified a pressing need for mental health workforce development to assist people with psychiatric disabilities to develop their skills and resources to get and keep work. This includes the requirement to give direct attention to the quality of employment opportunities available to people with psychiatric disabilities (Garske, 1999; Killen \& O'Day, 2004; Reddon et al., 1992). In addition, the literature recommends that attention should be given to job retention and job seeking skills, and integrated vocational pathways, thus creating a dynamic employment process that is more sensitive to individual need (Becker et al., 2005; Krupa, 2004; Gioia, 2005).

\section{Chapter Summary.}

In contextualising this return-to-work study, this chapter has looked back over the last sixteen years, to review, critique and synthesis the literature that arises from the existing body of knowledge. This review largely focused on current and significant international research into work, within both a mental health and socio-political context. This is in keeping with this study's critical hermeneutic 
design, wherein the interface between work, mental health and the employment environment was explored within this literature review in terms of cultural, historical and socio-political influences. As there is scant reference to employment within the New Zealand mental health literature, this chapter relied on government publications and the popular press to provide an overview of vocational rehabilitation in Aotearoa. A significant outcome of this literature review is it highlighted that there is a dearth of rehabilitation research, or even reference to vocational or other rehabilitative measures, within the forensic literature (Couldrick \& Aldred, 2003; Lindqvist \& Skipworth, 2000; Lloyd, 1995; Tracey, 2000). Accordingly, the international research findings and the current New Zealand employment literature have informed vocational rehabilitation practise from a forensic psychiatry perspective. 


\section{CHAPTER THREE: METHODOLOGY}

\section{Chapter Overview}

This chapter discusses the selection of a critical hermeneutic methodology in order to explore the return-to-work experiences of people who have had long term hospitalisation in a forensic setting. When discerning a research framework, there are four sequential elements: epistemology; theoretical perspective; methodology and methods (Crotty, 1998). Each element serves to inform the next sequential step within the research framework. The first three elements of the research framework are explored within this chapter. The epistemology informs the theoretical perspective, and both of these elements provide the guiding ideologies upon which this study's critical hermeneutic methodology is positioned. The methodology then governs the choice and use of the research methods which are explored in the following chapter.

Epistemology is the theory of knowledge that informs the theoretical perspective. As described more fully in this chapter's concluding paragraphs, epistemology within a critical hermeneutic inquiry is aligned with idealism, which postulates that knowledge is emancipatory (Crotty, 1998). The theoretical perspective, which rests upon the epistemology, is the philosophical stance that underpins the methodology. In this critical inquiry, the theoretical context that informs the research process is grounded in the logic of the critical perspective. According to critical logic, the social construction of reality is inherently about power and where people are positioned in relation to power (Grant \& Giddings, 2002; Smythe, 2000). This assumption drives critical researchers' socio-political agenda, which this chapter explores in-depth through the notions of critical theorist, Paulo Freire. His emancipatory constructs that inform this study are individually explained within this chapter. Hence, this chapter serves to outline the philosophical tenets and theoretical positions that underpin this return-towork study's methodology. 
Selecting a Critical Hermeneutic Perspective

People with mental illness frequently experience exclusionary social and employment practises, and therefore identify with other disenfranchised populations (Anthony et al., 2002; Marrone \& Golowka, 1999; Lapsley, 2003). When these individuals also have a forensic psychiatric history, they have even less access to employment and social opportunities. Therefore, they are one of the most excluded groups within western society (Joe, 2003). Therefore, I selected a critical hermeneutic approach for this study. This is because hermeneutics' focused on the informants' lived experience in order to generate a better understanding of the social, cultural, and historical context in which their return-to-work experiences occurred (Crotty, 1998). The critical lens then called the influencing socio-political and economic forces into question (van Manen, 1997).

An underpinning tenet of hermeneutics is the unavoidability of interpretation. It is borne of necessity that individuals construe the world, and explicate meanings from the objects and people in relation to the world (Heilman, 2003; van Manen, 1997). Central to the interpretive process of hermeneutics, is the premise that understandings emerge through communicative actions which are revealed through text and human experience (Maeve \& Vaughan, 2001). Hermeneutic inquiry enables mental health researchers to look beyond traditional symptom recognition and alleviation investigations, to revealing how people with mental illness interpret the world, within their socially and historically conditioned context. In terms of the critical perspective, critical hermeneutics is concerned with people in relation to their world from the outside in, rather than from the inside out as traditional hermeneutics envisioned. Hence, a critical hermeneutic approach involves an understanding of the ways in which perception and communication are unavoidably influenced by identity, and by socio-political and economic forces (Heilman, 2003; van Manen, 1997). An assumption that underpins both critical social theory and hermeneutic philosophy is that both reality and knowledge are socially constructed. Accordingly, knowledge and reality are constituted through communicative actions between people, and between the interpreter and the text (Maeve \& Vaughan, 2001). 
Selecting a critical hermeneutic methodology for this study allowed me to explore the complex interface between the health and employment sectors from the perspective of the key critical determinants; power and knowledge. A critical perspective recognises that the employment culture for people with mental illness is not separate from the give and take of everyday society (Grant \& Giddings, 2002). Therefore, the informants' experiences are seen to mirror the inherent contradictions and oppressions of daily life. An aligned critical assumption is that when disenfranchised people confront their reality, they discover in it the obstacles to their emancipation, and are therefore positioned to intervene in their situation (Crotty, 1998; Freire, 1972; Grant \& Giddings, 2002; Maeve \& Vaughan, 2001). The momentum for social change is thus able to be generated by the informants, from within their own communities, rather than coming from the researcher. The researcher, however, facilitates social change when they organise their findings in such a way as to imbue the informants with knowledge about the circumstances of their own lives (Burns \& Grove, 1997).

In regard to my selected data analysis approach, I extracted themes using van Manen's (1997) 'Researching Lived Experience' text to primarily inform my analysis. I found the focus on lived experience was useful for organising data in accordance with a critical hermeneutic epistemology. I also perceived that the inherent flexibility of van Manen's (1997) thematic analysis did not impose an intrusive theoretical framework that may distance the informants' influence, as their collaboration is desired in a critical inquiry. There is a dynamic and fundamental relationship between the knowledge generated by actual lived experience, and the trajectory of critical theory (Heilman, 2003). This action and reflection cycle that leads to lived experience being used as the initiator of social action, illuminates Freire's (1972) notion of praxis, which is a central construct in his theoretical perspective. As explained in the subsequent paragraphs, Freire's critical social theory underpins the theoretical construct upon which this study is positioned. 


\section{Freirean Critical Social Theory}

In accordance with the critical paradigm, this inquiry was linked to critical social theory. As people with mental illness readily identify with other disenfranchised populations (Marrone \& Golowka, 1999), I selected Brazilian critical theorist, Paulo Freire's liberatory education construct. His theories orientate the disenfranchised towards progressive emancipation and humanisation. For almost two centuries critical theorists, including Marx and Freire, have promoted a critical stance towards the investigation of oppression, which has been the historical institutional reality for people with mental illness. Marx provided a valuable platform upon which to critically view institutionalisation, the mental health paradigm that prevailed for over five hundred years (Ezersky, 1991; McCurrin, 1994; Schneider, 1998b; Vostanis, 1990). During the last two decades, however, the shift towards community integration has seen Freire's theories provide a valuable framework with which to critique the building of social capital and the promotion of social justice for people with mental illness (Maeve \& Vaughan, 2001; Powers, 2003; Schofield, 1998).

Previously marginalised within western societies, people with mental illness have gained increasing collective and individual self-determination over the last two decades (Marrone \& Golowska, 1999). As well as the transfer of power to mental health service users, access to open employment opportunities has increasingly become an expectation (Anthony et al., 2002; Marrone \& Golowska, 1999). This vision, however, requires disenfranchised people to have critical hope as it is an important ethical quality in the struggle for emancipation. Freire (2004) argues that it is necessary to employ serious and diligent political analysis to reveal opportunities for hope, and to address the barriers. In this regard, the methodology that underpins this return-to-work study supports the notion that the bond between knowledge and action is strengthened by reinstating lived experience as a valid basis for practical action (Freire, 1972; Heilman, 2003).

After visiting the USA in the late sixties, Freire (1972) argued that the third world is not a geographical concept, but is essentially a socio-political character. Hence, for disenfranchised people everywhere, Freire's pedagogical methods seek conscientisation and societal liberation. He believes liberation occurs 
through a social action process that provides people with the opportunity to examine and critique social political influences that affect their lives. This liberatory action is not about achieving power to dominate (Schofield, 1998). It is action that relies on shared power in order to act with others to effect change. Freire totally rejects the expert/passive recipient approach that finds solutions for people instead of with them, and then imposes these solutions on them. Instead, Freirean theory aims to move disenfranchised people from being the recipients of patronising solicitude, to being critics of the contradictions within their current reality, and ultimately to being creators of strategies for social transformation (Crotty, 1998; van Manen, 1997).

In 1970, Freire published a Marxist based critique of education called 'Pedagogy of the Oppressed' (Freire, 1972) which is the text that primarily informs my Freirean critique, although his last pedagogical text 'Pedagogy of Hope' (Freire, 2004) is also referenced. My selection of Freire's work was also driven by the relative accessibility of his language and theory, as my critical design required me to take my findings back to the informants for verification. Heilman (2003) states that a common criticism of critical theory relates to the tendency of critical theorists to use esoteric language and difficult writing styles. Hence, I desired theory and language that I could see resonating well with the informants, as well as with the broader mental health sector. This was to avoid limiting this study's audience, which could subsequently limit the political potential desired from a critical design. Despite my best endeavours to make Freire's work as accessible as possible to the widest possible audience (including informants, academics and health practitioners), Freire's language, like critical theory in general, can still be challenging. Therefore, these terms are explained in the following sub headings. This Freirean terminology has been used to critique my findings in order to add critical depth and explicate the social-political influences.

\section{Authentic Reflection}

Freire (1972) states that since humans are indivisibly linked to the world, the movement towards liberation must begin with the relationship men and women have with their world. Hence, authentic reflection is not concerned with abstract ideals or the world without people. Instead, it is critical reflection that is 
grounded in the here and now, and involves the situation in which disenfranchised individuals find themselves submerged (Crotty, 1998; Freire, 1972; Heaney, 1995; Smith, 2002).

\section{Alienation}

Freire's theories are led by a concern for the oppressed. These are the people who have been historically and culturally alienated within a regime of oppression imposed by those in power. Alienation was originally a Marxist term, and refers to the subjugation of the people by the social elites, resource constraints and the political forces of hegemony. In a situation of alienation, people may be impaired in their use of power. Thus ultimately, alienation interferes with the production of authentic thought and culture (Crotty, 1998; Freire, 1972; Heaney, 1995; Smith, 2002).

\section{A Sense of Project}

According to Freire (1972), humans are beings who move forward and look ahead with what Freire terms a 'sense of project'. As people transform their environment through human activity, they are fashioning culture. Consequently, new challenges and tasks continue to evolve within a never ending enterprise. It is the story of this ongoing interplay between humans and their world that constitutes human history and characterises men and women as essentially historical beings who have historical and value dimensions and 'a sense of project' (Crotty, 1998; Freire, 1972; Heaney, 1995; Smith, 2002).

\section{Transformative Action}

Freire (1972) suggest that the human world is not a static reality, but a reality in progress wherein people are the creators. Hence, men and women are called to transformative action in order to transform the human world, and thereby transform themselves. In constantly transforming their environment, people are creating and recreating the very conditions for their existence. This action, however, does not necessarily result in liberation. Freire also warns that an oppressor's transformative action can also dehumanise the world with their destructive presence. Accordingly, only the hindsight of history fully reveals the dilemma of being human and the consequences of having selected one path over the other. As people act upon the world, they transform it by their actions; 
consequently their consciousness is historically and culturally constituted. Hence, the transformation of the world is humankind's entry into history (Crotty, 1998; Freire, 1972; Heaney, 1995; Smith, 2002).

\section{Conscientisation}

Freire (1972) links conscientisation to the relationship between humans and their world, and to 'praxis' as a form of reflection that arises from, and remains indivisibly linked to, active human intervention in reality. Conscientisation is a process that involves identifying contradictions in experience through mutual dialogue. This ultimately leads people to break through prevailing societal myths, to reach new levels of awareness, and in particular an awareness of oppression. Rather than dwelling on the abstract, Freire states conscientisation is a joint project that takes place in real people and in actual social structures. This process is at the heart of liberatory education and action, and is an ongoing process by which an individual moves towards critical consciousness (Crotty, 1998; Freire, 1972; Heaney, 1995; Smith, 2002).

\section{Humanisation}

Humanisation is an emancipatory Freirean (1972) concept. This term encapsulates the quest to be more fully human by creating a humanly enhancing culture as a fundamental means of striving for liberation. This quest, however, can be negated by the manipulative forces of dehumanisation which compromise human rights for personal gain and power. According to Freire, when disenfranchised people confront the critical reality of their situation, they discover in it the obstacles to their humanisation and are then called to struggle against them. Any revolutionary moves against the oppressions of the status quo, however, also need to be humanised. This is so the new regime can confront and cast aside the tendency to replicate the oppressions of the former regime. Therefore, from a humanisation standpoint, the acquisition of power and knowledge in the quest for liberation, strives to liberate both the oppressed and the oppressors (Crotty, 1998; Freire, 1972; Heaney, 1995; Smith, 2002).

\section{Critical Consciousness}

According to Freire (1972), critical consciousness is characterised by an in-depth interpretation of problems. This level of mature critique occurs through testing 
one's own findings, while remaining receptive to further revision. Analysing problems at a critically conscious level also means avoiding the distortions caused by preconceived notions and being open to change, without rejecting traditional ways of being solely on the basis of age. Inherent in striving for critical consciousness is the rejection of passive compliance in order to embrace collaborative and dialogical processes. Consequently, critical consciousness is realised not only through an individual or intellectual effort, but through collective struggle and praxis. As people engage in the world as transformers of that world, individual and collective consciousness ensues. Therefore, critical consciousness in turn, leads to further action. In and through such action, people cease to see their situation as an enveloping blind alley. Instead, it emerges as a historical reality which is open to transformation (Crotty, 1998; Freire, 1972; Heaney, 1995; Smith, 2002).

\section{Praxis}

Praxis is a complex cyclical process of action-reflection-action upon the world in order to transform it. As praxis is central to the quest for liberation, it is an emancipatory process that leads to the development of an individual's critical consciousness. Therefore, self-determination, intentionality, creativity and rationality are the human characteristics that underpin the cyclical process of praxis. As this is an interdependent process, praxis cannot be divided into a prior stage of reflection and subsequent stage of action. According to Freirean thinking, reflection without action merely leads to being an armchair revolutionary. Conversely, action without reflection leads to unsubstantiated activism which is just action for actions sake. When action and reflection are united, however, they create culture and society by constantly and mutually illuminating each other (Crotty, 1998; Freire, 1972; Heaney, 1995; Smith, 2002).

\section{Dialogical Method}

According to Freire (1972), the only valid approach to liberation is the way of mutual dialogue. Both the oppressed and those in power must avoid the pitfall of pseudo participation in the dialogical process, as it requires committed involvement by all concerned parties. To foster participation of this kind, there must be trust in the oppressed and their ability to reason. Action and reflection in 
fellowship and solidarity is precisely what Freire means by dialogue (Crotty, 1998; Freire, 1972; Heaney, 1995; Smith, 2002).

\section{Problematisation}

Freire's (1972) problematisation is a pedagogical process that sets out the existential situation of those people who are concerned with a set of problems. To problematise is to engage a group in the task of translating the situational reality into meaningful symbols that are able to generate critical consciousness. In particular, attention is given to the interrelationship that these problems have with nature, and with oppressive socials forces. The oppressed have a blinkered view that the 'culture of silence' has imposed upon them. Therefore, they must propel themselves towards a view that sees their reality in its entirety, with all its causes and consequences. Accordingly, their problems are viewed as challenges, rather than outcomes of an intransient fate.

Problematisation is the antithesis of problem solving. In problem solving, Freire (1972) believes the problem solvers are often an elite group who maintain a distance in order to reduce the problem to objective dimensions, which are then conducive to practical remediation. Problematisation, however, recognises that solutions are often difficult because the wrong problems are being addressed. In particular, attention is given to the interrelationship that these problems have with nature, and with oppressive socials forces. In this regard, problematisation has a parallel process to demystification. In accordance with demystification, Freire (1972) stresses the need to problem pose the myths handed down to society by the oppressors. Problem posing, another key word in Freirean thinking, is an initial task within the problematisation process that allows all previous conceptualisations of a problem to be brought into question (Crotty, 1998; Freire, 1972; Heaney, 1995; Smith, 2002).

\section{The Culture of Silence}

It is uniquely difficult for people who are disenfranchised to become critically aware of their marginalised position, and intervene in their reality, because they belong to the 'culture of silence'. Freire uses this term because in their situation of oppression, the disenfranchised have no political voice, and they are unaware they do not have a voice. This is because disenfranchised people are not heard by 
the dominant members of their society, who in turn prescribe the words to be spoken by the oppressed through controlling society's dominant institutions such as churches, schools and government agencies. Consequently, the dominated have internalised the cultural myths of the dominators. Among the myths the disenfranchised have internalised, are the negative images of themselves. They come to see themselves as the oppressor views them, as incompetent, unreliable and so on. In this way, this mythology-driven exploitation engenders the disenfranchised's lack of self-determination and life aspirations. These are strategies that effectively silence the people who feel incapable of selfgovernance, and this can even lead to a fear of freedom. This is the 'culture of silence' wherein the oppressed are excluded from taking an active role in the social-historical transformation of their society and thus cannot exercise their right to participate consciously in the creation of their own destiny (Crotty, 1998; Freire, 1972; Heaney, 1995; Smith, 2002).

\section{Liberatory Action}

Liberatory action seeks to transform the social order by promoting participatory and democratised structures, while denouncing authoritarian and alienating systems of organisation. Freire believes that the power sharing and collegiality that occurs within, and as a consequence of, liberatory action, is far more protective of human rights that hierarchical modes of organisation. Hence, the governance of liberatory action is dialogical and egalitarian, as personal freedom and the growth of individuals can only occur in mutuality with others. Liberatory action essentially hinges on critical consciousness, and the development of the appropriate skills and competencies related to liberatory praxis. Freire expressed scepticism about the ability of western cultures to conceptualise empowerment as a collegial social act, instead of an individual characteristic. He viewed the term empowerment as a concept that was often subverted by those implementing preexisting intervention strategies in order to create illusions of choice, thereby disguising control as empowerment. Hence, Freire was cautious of using the word empowerment, instead referring to 'liberation' as he believed this term better reflected the notion of a collective emancipatory act (Crotty, 1998; Freire, 1972; Heaney, 1995; Powers, 2003; Smith, 2002). 


\section{Liberatory Education}

According to Freire (1972), traditional schooling is considered to contribute to the marginalisation of people who have historically been subjugated within their society because of culture, poverty or disability. Freire believes that education is never an ideologically neutral process. Adult education, whether remedial or for career advancement, generally replicates patterns of earlier schooling. This hierarchal mode of instruction tends to focus on respect for authority and good work habits. This contrasts with the Freirean experience of liberatory education, wherein the syllabus is centred on the learner's personal experience and pressing social needs. Freire states that this process protects the individual's rights and opportunities far more than authoritarian modes of learning that are underpinned by the alienating ideologies of the social elite. Education for liberation aims to challenge the socio-political nature of the world and enables learners to reflect on their historical and cultural experiences. It awakens in adult learners the expectation of change, a power which once awakened, seeks expression in transforming social action (Crotty, 1998; Freire, 1972; Heaney, 1995).

\section{Summary}

Freirean social theory has informed my critical hermeneutic research approach by critically examining the implicit rules and assumptions of the return-to-work experience within a historical, cultural, and political context. This includes using Freirean notions such as the 'culture of silence' and 'liberatory education' in order to analyse the constraints on communication and human action. In addition, Freire has provided the empancipatory processes of praxis, humanisation, conscientisation and critical consciousness. These constructs have enabled this study to identify both the contradictions within society, as well as the employment conditions that make authentic knowledge and action possible.

\section{Critical Epistemology.}

In designing my research framework, the theoretical perspective hinged on a certain way of conceptualising the ways of knowing in the world (epistemology) as well as a certain way of understanding what is the nature of reality (ontology) (Crotty, 1998). Epistemology within a critical hermeneutic inquiry is aligned 
with idealism, which postulates that knowledge is emancipatory. Hence, knowledge furthers self-determination by enlightening individuals about how they may rationally act to realise their own best interests (Burns \& Grove, 1997; Crotty, 1998). This assumption co-exists with the premise that finding out about people is best achieved when the relationship is not hierarchal, and the inquirer is prepared to invest his or her personal identity into the relationship (Rogers \& Palmer-Erbs, 1994; Schofield, 1998). Accordingly, I incorporated the key radical values of reciprocity, participation and power sharing into my methodology (Grant \& Giddings, 2002). In reflecting these values, I engaged in a collaborative process that helped structure the research question and continued to influence the research process throughout (Grant \& Giddings, 2002).

Ontology from a critical perspective deems that the social construction of reality is power laden (Grant \& Giddings, 2002; Smythe, 2000). Knowledge is generated within politically constituted relationships, between individuals and their concrete and cultural reality. This critical premise illustrates Crotty's (1998) assertion that ontological and epistemological issues tend to emerge together within the research methodology. Consequently, in critical designs, as with most qualitative research methodologies, it is difficult to separate the steps. In this return-to-work study, dialogue is used to both collect and to interpret the data; hence there is a constant movement between collection and interpretation. In accordance with the critical design, the conversations between me, as the researcher, and the informants required a relationship which involved power sharing and active reciprocity (Grant \& Giddings, 2002).

\section{Chapter Summary}

This chapter outlined the epistemology and the theoretical perspective's upon which this study's critical hermeneutic methodology is positioned. In this regard, this chapter provided a detailed explanation of Freire's (1972) social theory, which informs this study by adding critical depth to the findings. Having described the philosophical and theoretical tenets that underpin this return-towork study's methodology, the next chapter details the strategy that lies behind the choice and use of the research methods. 


\section{CHAPTER FOUR: METHOD AND RESEARCH DESIGN}

\section{Chapter Overview}

This chapter details the research method utilised within this return-to-work study, and then explains the procedures by which this research was conducted. Research methods are the techniques and procedures used for gathering and analysing data related to a research question (Crotty, 1998). The research design is the overall plan for addressing the research question, including specifications for enhancing the integrity of the study (Polit, Beck \& Hungler, 2001). As described in the preceding chapter, the choice of research methods and design was governed by the critical hermeneutic methodology. In outlining these procedures, the aligned critical hermeneutic processes of self-reflexivity and collaborative processing are also detailed. Also highlighted are the challenges encountered in bringing this research design to fruition.

Following a brief introduction to the informants and the recruitment strategies, this chapter focuses on the selected data collection and data analysis techniques. In detailing the data analysis strategy, which was primarily informed by van Manen (1990), I describe the custom building and choreographing of a set of processes which had a 'goodness of fit' with my research question. Particular attention is paid to describing the final step in the analysis. This is the development of the essential themes that contain the 'bones' of the return-towork experience, and through these the collective voice of the informants is expressed. This chapter then concludes by describing both the rigour and ethical requirements that have been addressed since this study's inception.

\section{Method}

\section{Sampling Strategy}

A purposive sampling strategy was employed as it was consistent with my selected research design. In handpicking my informants, I based my selection on both my and the intermediaries' (who are described in detail p. 55) background knowledge of the informants' ability to articulate and reflect on their experiences 
(Denzin \& Lincoln, 1994; Polit et al., 2001). This sampling strategy also enabled me to select eight individuals were able to offer alternative viewpoints, from different social, cultural and employment positions, in order to attain a richly textured and comprehensive set of data.

\section{Profile of Study Informants}

All of the informants' life experiences aligned with the following inclusion criteria:

- They were hospitalised within a forensic setting for three years or longer within the last 15 years.

- They had been discharged from a forensic inpatient setting for at least six months, and were living in their own, or in a family living situation, or in community-based supported accommodation.

- Within the last decade, they have returned to part or full-time employment for a period of at least six months, and received tax related earnings as remuneration.

- They are over 20 years old and able to communicate effectively in English.

The only exclusion criterion was if people were currently experiencing acute or severe symptoms of their psychiatric illness, this was not the case for any of the selected informants.

Confidentially is of the utmost importance when working with the population under study, as some of the members may still be recipients of unwarranted media scrutiny, and continued victim grievance. It is imperative that the demographic and workplace information contained within this report has no identifiable features. Accordingly, the following demographic information deliberately profiles the informants in collective terms, with generalised rather than specific details providing an overview of the group under study.

In line with this study's critical hermeneutic design and the requirements of a Masters thesis, prior ethics approval from the Ministry of Health Regional Ethics Committee had been granted to recruit between five to eight informants. 
Therefore, four men and four women whose ages ranged from twenty eight to fifty six years old were recruited to join this study. Four of the informants resided alone in their own or rented accommodation. One person was living in a community based mental health residential facility, and all the others lived with partners or friends in rental accommodation. Six of the informants were employed within an open employment position at the time of interview. The other two people had resigned from their previous positions, and now had parttime positions within consumer-driven affirmative businesses (as described on p.117). Both people held positions of responsibility within these operations and their hourly earnings closely equated with the financial remuneration they would receive in open employment positions. Four of the informants were Māori, two were Pacific people, one of whom was born in New Zealand, and the others were all New Zealanders of European descent.

In accordance with the DSM-IV ${ }^{10}$ psychiatric diagnostic criteria, all the informants had being diagnosed as having an Axis I clinical disorder, including schizophrenia, bipolar affective disorder and schizo affective disorder. As illustrated within the quoted extracts, five of the informants also presented with co-existing substance abuse issues on their initial admission to forensic services. In all cases, this dependency had been addressed during their years of secure inpatient care and had not presented as a concern since their subsequent discharge into the community. For all the informants, their forensic admission had been heralded by a specific index offence that occurred when they were acutely mentally unwell. These offences ranged in severity from crimes against property to violence towards others, and included the serious end of offending

The length of time the informants had lived in the community post-discharge varied from eighteen months to eight years two months, with all but one of the informants being in the community for at least three years. During this period of time, no one had required re-hospitalisation within either forensic or general psychiatric services. All the informants at the time of the interview were under

\footnotetext{
${ }^{10}$ The Diagnostic and Statistics Manual Disorders (DSM) is the diagnostic tool for mental health disorders that is most commonly used within New Zealand mental health services. It is subject to regular revision, and is currently in its fourth edition (American Psychiatric Association, 2000).
} 
some form of compulsory treatment order pursuant to either the Mental Health Act (1992) or the Criminal Justice Act (1985). Four of the informants were still receiving follow-up community care from forensic services. The other informants had been assessed as no longer requiring this level of specialised follow-up and had been transferred to general community mental health services.

\section{Intermediaries and the Recruitment Process}

The small pool of prospective informants living in the two identified New Zealand regions, combined with my lengthy experience in the field, meant some of the prospective informants were known to me, although not necessarily in terms of a direct therapeutic relationship. Because this acquaintanceship may have led to people feeling pressured to participate, and the fact that this is a vulnerable population under study, I used intermediaries to directly approach people. Following the advice of the relevant service managers, the intermediaries I selected were: Maori and Pacific Island cultural advisors; a hospital chaplain; and clinical and rehabilitation practitioners working within either the selected District Health Board (DHB) or Non Government Organisation (NGO). Both services had a forensic service delivery contract.

The stepwise process of selecting and approaching intermediaries was part and parcel of formally presenting my research proposal through the different management tiers. I made my initial approach to the respective service and rehabilitation managers of both services in order to formally present my research proposal. During this initial meeting, the appropriate managerially-designated team leaders were identified. I then contacted the relevant cultural, community and rehabilitation team leaders who proved names of possible intermediaries. My subsequent approach to the twenty one prospective intermediaries occurred both verbally and in written letter form (see Appendix G). Following this initial approach, conversations with six of the intermediaries led to mutually identifying prospective informants. The intermediaries subsequently approached these people and provided general information about the study. The prospective informants were asked for expressions of interest to be either channelled through the intermediaries, or to ring me directly. Initially, I underestimated the time requirement involved in incorporating intermediaries into my research design. 
This was related to both the number of intermediaries I eventually approached (twenty one), and the length of time the process took from the initial intermediary approach, to actually recruiting the informants. This timeframe varied from one week to eleven months. Overall, using intermediaries was protective for the informants, and provided me with the support and expert procedural guidance that enabled me to move forward with confidence, albeit at a slower pace than initially predicted.

Over a period of eleven months, ten prospective informants were approached, with eight readily agreeing to participate. Following the informants' expression of interest, I provided a full explanation using the participant information sheet as a guideline (Appendix A), during a prearranged meeting either at their home or at my place of work according to their preference.

\section{Setting}

In each instance, the interview setting was selected by the informant with the goal of reducing the interviewee's anxiety, thereby producing more informative interviews within a reciprocal process (Rogers \& Palmer-Erbs, 1994). Five of the informants chose their own home for the interview. The others choose to meet with me away from their living environment, as the interview space I was able to offer was a more private option. This space was conveniently and anonymously located, in a new community setting with no mental health signage. In all cases, the informants selected the time of the interview to fit around their work commitments, which included them nominating evenings and weekends.

\section{Data Collection}

\section{The Interviews}

The first step in the interview process was a pre-interview meeting with each of the informants to clarify the research process and gain informed consent. These unstructured meetings also provided me with the opportunity to connect with the informants, thereby establishing an ongoing mutual rapport that would ultimately maximise the meaning of the informants' narratives. My pre-interview preparation was well considered from the outset. Aided by a checklist, equipment 
and procedural pre-checks were done in order to avoid placing any undue burdens in terms of time, or unnecessary stress, on the informants. All the informants were encouraged to have a support person with them during the interview. An outcome of previously consulting with the forensic cultural services was that Maori and Pacifica cultural advisors had made themselves available for this role. Two of the informants who knew their respective cultural advisors well, chose to have them involved in the interview process, whereas another informant chose to have a member of her whanau join her.

The unstructured interview process within a phenomenological inquiry aims to explore and gather personal stories in order to develop a richer and deeper understanding of a human phenomenon (van Manen, 1997). In this study, the primary data collection occurred within an audio-taped in-depth conversation. While adhering to the unstructured nature of the interview process, I utilised a brief interview guide that consisted of succinct, clear, open-ended interview prompts (Appendix E). These non-directive openers allowed the informants to tell their stories in a naturalistic, narrative fashion within sessions that varied from 45 mins, to an hour and 45 mins in length. In order to gain critical depth during the interviews, I had previously developed and documented a conceptual framework based on the work of critical researchers, Heilman (2003) and Jones (2000). This framework placed aspects of the return-to-work experience within a socio-political and economic context (Appendix F). The purpose of this framework was to enable me to explore both the obvious and more subtle social, political and economic dimensions, if and when, the informants raised these aspects of their return-to-work experience. These procedures enabled the informants to determine the interview content, and therefore provided the opportunity for them to engage in a self-reflective inquiry. Therefore, the critical hermeneutic goal of increased self awareness and self efficacy through the direct experience of the interview process was realised, to varying degrees, by all the informants (Crotty, 1998).

\section{Journal Data}

As consistent with a hermeneutic inquiry, observations of events and the physical environment were incorporated as journal data (Koch, 1996). This diarising 
provided a context for the informants' narratives and consolidated my understanding of the setting. Each interview was recorded within my journal using the following headings: Setting up the interview; My observations of the non- human environment; My observations of the interviewee, My responses to

the interview experience; Koha and Follow-up contacts. The interrelationship of this journal data with other data generated within this study is further explained under 'data triangulation' in the rigour section of this chapter (see p. 67)

\section{Transcriber}

Prior to commencing the interviews, I had decided to employ the services of an experienced transcriber who was familiar with the process of detailed verbatim transcribing. The transcriber I sought out had both the required transcription expertise as well as a clear understanding of the rigorous confidentiality protocols due to her experience in the area of forensic psychiatry. The transcriber signed a confidentiality agreement (Appendix D). On completion of each interview I hand-delivered the audio-tapes to the transcriber. When the tapes had been transcribed verbatim, the tapes and the electronic transcripts were returned to me for checking and data analysis. The management and secure storage of the tapes and electronic interview records are described in the 'ethics' section of this chapter (see p. 77).

\section{Follow-up Interview Procedures}

The initial interview was followed up by one or two visits to the informants, depending on their stated need, in order for me to return and discuss the typed transcripts. Therefore, each informant had an opportunity to clarify issues arising from the first interview, comment on their transcripts and remove any information they wished. As I had already engaged on some initial data analysis (see p. 61), the informants were also in the position to comment on my early interpretation of their experiences. In my journal I documented two of the informants' verbatim comments about how well they thought they had done during the interview, in light of the retrospective view the transcripts had provided: "The interview was much better than I thought it would be" (Lavina, 19.05.06). "Sounds good... better than I thought" (Joe, 07.06.05). 
As consistent with my documented ethics application, I contacted the informants' key workers following the interview and I rang each of the informants the day after their interview to check on their post interview responses. In all cases, the informants responded that they had not been emotionally upset following their interview. Maria acknowledged, however, that during the interview she was aware of her emotional responses to the content. This follow up was documented in my journal notes as demonstrated in this excerpt which I recorded after Maria's second interview:

Maria did acknowledge that she had felt emotionally stirred up during the interview, but that it felt $O K$ now it was over and she hadn't been dwelling on any of the content. She reiterated that when I checked in again the next day. Her key worker also perceived that Maria had emotionally managed the interview without any negative impact. Maria also stated after this, the second interview, that the interview process had been helpful in reflecting positively on the experience, and on how the step by step nature of getting the job she eventually desired, had unfolded. (Maria, 14.04.05)

\section{Data Analysis}

\section{Selection}

Qualitative data analysis is initially guided by the research question. As I was engaged with an emergent process, however, the specifics of the data analysis evolved over time as my project progressed. In contrast to quantitative research, qualitative data analysis is less standardised and has historically been less explicitly outlined by researchers. (Neuman, 2003; Sarantakos, 1994). Because data analysis was the area of my research that I was initially most apprehensive about, I spent many hours progressing my way through qualitative research texts, in order to understand the ethos behind phenomenological data analysis. My reading quickly led me to the realisation that qualitative data analysis is not able to be prescribed by a set of explicit rules and principles that can be conveniently replicated from study to study. Instead, I was required to custom build a set of conceptually specified analytic processes that had a 'goodness of fit' with my research question, positioned within the critical paradigm. 
While there was an impressive quantity of material on qualitative research, it soon became apparent that a very small percentage of this literature was able to practically guide my analysis from a hermeneutic or critical perspective. As I honed my notions about which phenomenological data analysis approach aligned with my research question, I looked at the three schools of phenomenology. Although a rather simplistic encapsulation of the small but detailed body of writing about phenomenological analysis, my synthesis of the literature and my decision making was assisted by Polit et al. (2001) description. They outline how the different schools of phenomenology have developed three unique approaches to data analysis. The Duquesne school of phenomenology is the first approach as it arises from the inaugural school based on the methods of Husserl's philosophy. The second school is the Dutch Utrecht School, and it is this approach which primarily informs the data analysis in this study. Phenomenologists who use the Utrecht approach combine characteristics of the descriptive and interpretive phenomenology. The third school of phenomenology is an interpretive approach called Heideggerian hermeneutics.

From the Utrecht school, van Manen's (1997) method is an example of the combined approach that blends the notions of both Husserl and Heidegger. In addition, whereas hermeneutic phenomenology has often been discussed as a mere descriptive or interpretive methodology, van Manen (1997) argues that it is also a critical philosophy of action. In qualifying his assertions, van Manen states that hermeneutic phenomenological reflection engenders a depth of thought that can radicalise the stakeholders' perceptions. The depth of critical reflection that phenomenology sponsors is more likely to lead to a perception of social injustice than other interpretive or positivist methods. Van Manen (1997) purports that his approach to data analysis may prompt the researchers, readers and informants' to political action, and therefore aligns with my research question.

While my analysis is largely informed by van Manen's text, 'Researching Lived Experience' (1997), other qualitative research authors also provided me with valuable insights and practical strategies to guide my journey. Sarantakos' (1994) and Neuman's (2003) texts provided me with a valuable body of thought about the theoretical and methodological principles that underpin qualitative data 
analysis, including references to phenomenological analysis. In addition, Burns and Grove (1997) offered me a valuable critical lens with which to view data analysis. My critical approach to data analysis was also enhanced by Tolich and Davidson's (1999) field work research perspectives which added depth to my knowledge from both a New Zealand and informant-centred perspective.

My selected theorists, van Manen (1997) and Freire (1972) both share a guiding premise that is illuminated within the critical hermeneutic perspective that informs this study. Both theorists concur that the bond between knowledge and action is strengthened by acknowledging lived experience as a valid basis for practical and political action. Therefore, I engaged with the data in order to illuminate Freire's (1972) and van Manen's (1997) critical theories about the social world within my analysis.

Analysis

Despite competing and polemic arguments, qualitative researchers agree that analysis involves a number of elements that are all related in an interlinked process that allows for the establishment of conclusions (Huberman \& Miles, 1998; Neuman, 2003). Sarantakos (1994) encapsulates these broad areas of consensus thought within the three following points. Firstly, analysis occurs within a cyclical continuous process that goes through data reduction, data organisation and interpretation. These linked sub-processes will be explored within the following two subheadings. The second area of consensus is that this cyclical process is lengthy and diverse. This includes the time I spent directly engaged with the informants and the data, as well as the self-reflective time I spent between sessions just dwelling with the data. The third and last point of agreement is that analysis and collection of data occur simultaneously. As described in the subsequent paragraphs, my data analysis took place before, during and after data collection (Sarantakos, 1994).

As I continued to inform and engage with my data analysis, I became aware that rather than conceptualise it as an unfamiliar and distinct component of my research process that it was demystifying to see it as it exists; a dynamic and interactive process. As Burn and Grove (1997) argue, I needed to critically explore the dynamic interactions between myself and the data during the 
analysis. This interaction occurred when the data was communicated through the written word, for example when making margin notes on the transcripts, and also in person to person contact during the interviews and associated feedback forums. The critical thinking used to examine this interaction is referred to as reflective thought or reflexivity.

\section{Data management and reduction}

Data management and reduction are interlinked analytic processes, which commenced with my choice of research question and conceptual framework. These early decisions were methods of anticipatory data reduction; essential analytic processes that heralded the commencement of my data analysis (Huberman \& Miles, 1998). The following paragraphs describe the data management and reduction processes which I employed to both guide and support the rigour of my data analysis.

As consistent with a qualitative methodology, soon after I commenced my research, the rapidly increasing and vast amount of raw data dictated the need for me to devise a systematic, coherent process for data collection, storage and retrieval. In addition to developing a detailed colour coded system of folders and matching box files to store the raw data and reference materials, I also maintained a handwritten system of diaries and notebooks. Within the former I documented all practical arrangements made with the informants. I also used notebooks to record the academic contacts which helped inform my study in the terms of writing groups, hermeneutic groups and the supervision process. In addition, my personal reflections directly related to the interviews and follow-up meeting with the informants were kept as data within an electronic journal.

Following each interview, I focused on familiarising myself with the data gathered by listening to the audio-tapes. Subsequently, I read and re-read the interview transcripts until I became immersed in the data, noting the informants' nuances and inflections within the text. This listening and reading heralded the next step of the data reduction process as I began to attach meaning to elements in the data. I made reflective margin notes which included my thoughts and insights as they gradually emerged into my consciousness. These remarks often connected the transcripts with other parts of the data or suggested new 
interpretations. Making these marginal notes also assisted me to remain connected with the data and retain a thoughtful stance while reading and rereading the transcripts. Subsequently, these margin notes were extracted and used for memoing within my analytic memo which I describe in the following paragraph.

In order to practically guide my memo recording, I used Neuman's (2003) prescribed analytic memo format to diligently keep a decision trail of the data analysis process. In line with Neuman's notions about analytic memoing, mine contained my critical reflections about the data, the analytic process and the emerging themes. As memoing is a dynamic process, I recorded my insights related to emerging theme with each pass I made through the raw data. In addition, I carefully attended to the analytic memo tracking requirements. These involved organising and reorganising data under draft thematic titles and a numbered recording system in order to connect the raw data with the emerging themes. Therefore, my analytic memo served to forge a link between the raw data and my more abstract, theoretical thinking. As my theorising progressively organised the raw data into conceptual categories, each emergent construct become the basis of a separate memo. Within these separate file memos, my discussion on each the emerging theme was complemented by selected narrative excerpts that illuminated the essential thematic meanings. Each of these memos subsequently formed the basis for my data analysis, as well as being integrated into the writing and rewriting of each essential theme in preparation for my final report.

As consistent with the cyclical nature of the data collection and analysis processes, I carried out early and interim analysis after my first three interviews. This initial analysis enabled me to focus much of the subsequent data collection on emergent constructs, while collecting additional data during the remaining interviews. Ongoing cyclical analysis is inflationary. Like Neuman (2003), I also found that the more I investigated, the more layers of meaning I discovered. As a researcher, however, I needed to engage in data reduction processes in order to see a reduced set of raw data as a basis for thinking about its meanings. The data management and reduction processes resulted in a reduced set of raw data as 
information was condensed, clustered, sorted and linked over time, throughout the study. Hence, my truly inspirational analytic moments were relatively brief, with these insights slowly fermenting throughout the weeks and weeks I spent engaged in mechanical data processing.

\section{Phenomenological Themes}

Thematic analysis refers to the process of revealing the themes that are embodied and illuminated in the evolving meanings and imagery within the text. Formulating a thematic understanding is not a rigidly prescribed process, but a fluid act of insightful creation and explication. In that regard, van Manen (1997) argues that lived experience is actually too dynamic and complex to be fully captured in conceptual abstractions. Ultimately, however, he concedes that the concept of a theme gives phenomenological researchers a conceptual framework upon which to position their research findings. Hence, the data collection, the

final text and theoretical issues that generated the themes are integrally linked within the creation and redefinitions of the research themes (Tolich \& Davidson, 1999).

Phenomenological themes may be understood as structures of experience (van Manen, 1997). Hence, van Manen's method of analysing lived experience informed my interpretation as I endeavoured to determine the experiential structures of the return-to-work phenomenon. According to van Manen, themes can be revealed and isolated from the informants' descriptions of experience by three different means as described in the following sentences. The wholistic or sententious approach is centred on an astutely constructed concise statement that seeks to interpret the meaning of the text as a whole. In the selective or highlighting approach, the researcher explicates the statements and phrases that seem the most revealing about the phenomenon. According to the detailed or line by line approach, the researcher analyses every sentence to explicate the meaning that they hold about the experience under study.

Having read and re-read the interview transcripts a number of times during the transcribing process, my initial foray into analysing the interview transcripts was aligned with van Manen's (1997) highlighting or selective approach. Accordingly, I re-read through the transcripts, and highlighted the statements that 
struck me as being the most revealing about the experience under study. I then used these highlights as reference points for my next analytical step, the wholitic approach. I found that the highlighted phrases captured my attention as I sought to grasp the essential meaning of each interview text as a whole. Accordingly, I attempted to encapsulate the fundamental meaning behind each of the informants experience within a wholistic statement as portrayed in the following example:

Returning- to- work is a re-entry to the world; a role shift that leads to a more equitable communion with friends and family, and while highly desired, this move requires psychological and physical fortitude. (Sam, 14.04.05)

Once the wholistic statements and highlighted phrases were identified, they became objects of reflecting and interpreting through the follow up interviews with the participants (Koch, 1996; Crotty, 1998).

After the follow up interviews, my third approach to the analysis was van Manen's (1997) detailed, or line by line approach, where each sentence was examined for the meaning that they hold about the return-to-work phenomenon. After all the data was grouped into meaningful clusters, I undertook a sorting process whereby less relevant or repetitive data was set aside. The remaining quotes and narratives were then relegated to the most appropriate emerging theme in a delineation process that was based on trial and error. As described by Burns and Grove (1997) my initial intuitive thematic forays were followed by careful examination of the data in order to verify the validity of my intuition.

Themes are reflexive and therefore change continuously throughout the course of the data analysis process (Tolich \& Davidson, 1999). Hence, a successive drafting process underpinned the refinement of the themes, with each literary pass adding in some way to the final text. This was achieved either by reorganising themes or winnowing out less productive themes, while focusing on the fruitful themes. Because successful drafting is a gradual, incremental and hesitant process, I resisted the desire to commit to premature explanations (Tolich \& Davidson, 1999). I also aimed to be cognisant of what points of view were not being considered within the text (Neuman, 2003). Hence, remaining open to disconfirming evidence while I was on my quest to capture the themes required me to not only discover the informants' commonalties, but also to 
search for experiential variations and omissions (Burns \& Grove, 1997). I then verified the themes by checking results with the informants and other experts in the field in member check and peer debriefing sessions respectively (these methods are explained in the subsequent rigour section, under the heading of 'credibility' p. 67). In addition, my data analysis was overseen by two thesis supervisors and portions of the data were jointly analysed to check for agreement between researcher and supervisor. A certain degree of researcher bias is acknowledged in critical research, but my supervisors assisted in verifying my interpretation to ensure the analysis was conducted as rigorously as possible.

The final step in my analysis was the development of the essential themes which contain the 'bones' of the return-to-work experience. These thematic explanations reveal the essence of the phenomenon, and therefore aim to be rich in detail, sensitive in context and capable of showing the complex processes of lived experience. Some of the themes are expressed in metaphors such as 'turning your life around'. Metaphors have value when expressing the essential themes as they embody a strong image that resonates with a feeling tone, which is powerful enough to add depth and meaning to the findings. Burns and Grove (1997) and Sarantakos (1994), however, have a cautionary note about metaphors, suggesting it is unwise to look for metaphors prematurely, or to over use metaphors as they can be taken too far in terms of meaning.

\section{Summary}

Hermeneutic analysis provides a dynamic, systematic approach to interpreting text. Initially, I analysed the whole text, and then parts of the text, comparing the two interpretations for conflicts, and for understanding the whole in relation to the parts and vice versa. This dynamic process of moving between the parts and whole of the text constitutes the hermeneutic circle in which the informants, I as the researcher, and even the reader of the report, endow meaning and understanding (Koch, 1996; Crotty, 1998). In addition, the themes seek to immerse the reader in the informants' world, while simultaneously persuading themselves of the researchers' authenticity and focusing the attention on the research question (Tolich \& Davidson, 1999). 
Through the custom built, dynamic, step by step analytic process described in the preceding paragraphs, eleven essential themes were eventually discovered (Koch, 1996; Crotty, 1998). As this study was positioned within the critical paradigm, I tried to conceptualise the essential meanings of the informant return-to-work experience by asking critical questions of the data. These questions were driven by the notions associated with Freire's (1972) critical social theory as discussed in the preceding chapter. My analysis therefore enabled an examination of both macro and micro levels of power involved in the informants' return-to-work experience through the multiple positionings of psychiatric disability, employment status and social capital.

\section{Data Quality}

\section{Rigour}

In order to contribute to knowledge research must be trustworthy and believable (Koch, 1996). Therefore all research must address the issue of rigour, ensuring that the selected procedures are compatible with each study's unique philosophical and methodological tenets. Rigour in qualitative research is associated with openness, philosophical congruency and procedures designed to enhance the trustworthiness of the data (Burns \& Grove, 1997). Lincoln and Guba's (1985) four criteria for establishing the trustworthiness of qualitative data: credibility; dependability; confirmability and transferability are used as paragraph headings in order to explore the rigour requirements determined by my research design. As these are generic qualitative rigour requirements, under these headings I have also incorporated Denzin and Lincoln's (1994) three rigour criteria as additional sub headings. These criteria are specifically designed for the critical paradigm: portrayals of constructed realities; catalytic validity and anticipatory accommodation.

\section{Credibility}

Credibility, the first rigour criterion, refers to the trustworthiness of the findings as judged by both the informants and people within the discipline (Schneider, Elliot, LoBiondo-Wood \& Harber, 2003). Lincoln and Guba (1985) assert that the credibility of an inquiry involves a two pronged approach. Initially the 
investigation is conducted using procedures that enhance believability, and then subsequent measures are taken to demonstrate credibility; procedures which are described in the following headings.

Prolonged engagement.

Prolonged engagement required me to invest sufficient time in data collection activities in order to acquire an in-depth understanding of the informant's world, and also to be able to test for misinformation (Polit et al., 2001). While I was mindful of not placing an additional burden on the informants in the terms of their time, I had face to face contact with each of them on at least two occasions both prior to and after the audio-taped interview. As described in the following 'member check' criterion, all the informants were invited to a presentation of this study's findings in July 2006.

\section{Data triangulation.}

Data triangulation refers to the multiple methods and perspectives used to collect and interpret data about a phenomenon, in order to distinguish trustworthy data from information with errors (Polit et al., 2001). Firstly, the hermeneutic construct 'fusion of horizons' required me to determine the horizons of my background knowledge and my preunderstandings, which I subsequently documented in a precommencement statement (Koch, 1996). Getting into the hermeneutic circle fully required me to incorporate my own experiences as data, and to be aware of my own subjective claims and references. By using the informants' dialogue excerpts to illustrate each of the emergent themes, I have sought to demonstrate that the data has been accurately interpreted and conceptualised. In line with data triangulation concepts, I have employed multiple data sources. These include: journal entries; conversation with the informants; peer debriefing notes; interviews and data from observations. Utilising multidimensional sources, serves to produce the best construction of the informant's experiences in a hermeneutic inquiry, and contributes to the trustworthiness of the research process (Koch, 1996).

\section{Peer debriefing.}

Peer debriefing sessions were held with peers to review and explore various aspects of the inquiry. Firstly, the AUT thesis supervision process ensured that I 
had professional mentoring and that I was exposed to the philosophical and methodological challenges of my supervisors. Both of my thesis supervisors are experienced in the hermeneutic perspective and the phenomenon under study. The forensic psychiatry Māori services manager and Pacific Island services manager both provided cultural advice at the outset, which included offering consultative support throughout the research process. As well as ensuring cultural safety during the interview process, I was able to seek advice and engage in some specific problem solving on how best to present the findings from a cultural perspective. Some of these issues and dilemma's occurred during the documentation of the findings and included issues such as the importance of financial contributions to family members from a Pacifica perspective.

Thirdly and finally, I also co-opted a peer debriefing group. Members of this group had expertise in an aspect of the phenomenon under study. Hence, this group consisted of a consumer consultant with lived experience of the issue, and a NGO Employment Consultant; a mental health Occupational Therapist/Career Advisor and a Forensic Rehabilitation Manager. The latter three people all had direct experience with the job seeking endeavours of people from forensic services. As well as one to one contact with individual members of this peer debriefing team as the themes were uncovered, the essential themes were emailed for both individual review prior to being discussed and affirmed within a group session.

\section{Member checks.}

Member validation involves seeking feedback from study informants to ensure the study findings are reflective of their experiences. Lincoln and Guba (1985) consider member checking the most important technique for establishing the credibility of qualitative data. Accordingly, I provided informal feedback to the informants regarding preliminary findings and interpretations in order to secure their initial reactions during our follow-up session. Once all the data was collected and analysed, I formally presented the findings, both verbally and in writing, within a group 'brunch' forum to which all the informants were invited (see Appendix H). Five people attended and one other elected a one to one session. Two people sent their apologies due to prior commitments. During this 
forum, the eleven essential themes were explained from both an interpretative and a Freirean perspective, with the informants' reflections and own interpretations being documented by a designated note taker. These notes were then incorporated as a data source.

\section{Portrayals of constructed realities.}

Denzin and Lincoln (1994) use their first criterion for establishing critical trustworthiness, 'portrayals of constructed realities' to further develop the concept of member checking within the critical paradigm. Accordingly, this study's credibility required the return-to-work constructions to be affirmed by the informants who constructed them. The informants' qualification of the eleven themes, and the meanings encapsulated within these themes, occurred during the individual and group presentations as described in the preceding paragraph. During these presentations, however, I needed to be cognisant of the potential for disagreement between my interpretations, and the informants' own perceptions. While individuals did not experience all aspects of the phenomenon, the eleven themes resonated with their wider understanding of the return-to-work phenomenon. Denzin and Lincoln (1994) caution that measuring trustworthiness in critical research can be challenging. This is due to the effects of the "culture of silence' wherein the power-over issues may not be perceived by the informants (Freire, 1972).

\section{Searching for disconfirming evidence.}

Searching for disconfirming evidence involves a systematic search for data that challenges an emerging theme (Polit et al., 2001). In the first instance, this process occurred through this study's purposive sampling. Handpicking my informants enabled me to select individuals who offered some conflicting viewpoints, from different social, cultural and employment positions. This strategy, along with prolonged engagement and peer debriefing (both previously described in this rigour section), greatly strengthened the comprehensive description of the informants return-to-work experiences

\section{Researcher credibility.}

Researcher credibility is endowed on a researcher, based on his or her training, qualifications and experience (Polit et al., 2001). As I was the data collecting 
instrument, as well as the creator of the analytic process, chapter one of this research report contains information about my credentials, and about the personal connections that I have to the topic, and to the community under study. While I needed to secure professional confidence in my data, research conducted from a critical perspective brings an inherent political agenda for change; enabling the disenfranchised to have decision making powers (Grant \& Giddings, 2002; Smythe, 2000). Therefore, I also needed to establish my credentials with Māori, Pacifica and mental health consumers within the peer debriefing and member check forums as described in preceding rigour paragraphs.

\section{Catalytic validity.}

Denzin and Lincoln's (1994) second rigour criterion stands alone, as a heading specific to the critical paradigm. It points to the degree to which this study moved the informants to understand the world, and the way it is shaped in relation to the politics of oppression and power. These understandings then lead to transformative action (Freire, 1972). In order for this research to display catalytic validity it needed to demonstrate that it promoted the informants' selfunderstanding and self-direction. As described in the data collection section of this chapter (see p. 56), this process occurred during critically self reflective moments in the informants' interviews. During the group 'member check' session (as described previously in this rigour section), issues such as the relationship between Freire's notion of the 'culture of silence' and the length of time people tolerated being the recipient of workplace bullying were well received by the informants, particularly the people who had been affected by this issue.

\section{Dependability}

Qualitative research is constantly examining and interpreting data and making decisions about how to proceed based on what has already being discovered. Accordingly, the second rigour heading, dependability, refers to the data stability over time, and within different conditions (Polit, et al., 2001). An inquiry audit involves scrutiny of the data and relevant supporting documentation by an external reviewer. This approach is interwoven with data confirmability. In the next paragraph, this rigour criterion is described in terms of the assembled audit 
trail of the steps taken to ensure data stability throughout the duration of this return-to-work study.

\section{Confirmability}

The third aspect, confirmability, is judged by the adequacy of information leading to the reader's ability to audit the researcher's influences and actions throughout the entire research process (Koch, 1996; Polit et al., 2001). Based on six audit trail steps identified by Polit et al. (2001), and incorporating work by Koch (1996), I collated a systematic collection of documentation as follows:

1. Raw data: This includes the interview transcripts and handwritten field notes which provide a dynamic record of research events.

2. Data collection and analytic procedures: These records are held within an analytic memo (as described within the data analysis section of this chapter, see p. 62) which records the method notes and the systematic development of the essential themes (Neuman, 2003). In addition, the analytic memo contains reference material related to the theorists and writers who have informed the data analysis.

3. Process notes: These records consist of extensive, selected references and personal notes that I have used to inform my theoretical perspective, and validate my methodologic rationale. Data from the member check and peer debriefing sessions are also included in these records.

4. Materials related to my influences and actions: These include my handwritten diary entries recorded following each informant contact. These notes were subsequently used as the foundation for my journaling notes. In this electronic journal I recorded my personal observations and responses to the interviews, the interviewees and the context in which they occurred.

5. Schedule development information: This includes a record of the interview schedule drafts, and drafts of the interview framework I developed in order to inform my interview procedures from a critical perspective (Appendix F)

6. Data reconstruction products: This includes all the drafts of the final report. 
Similar to a financial audit, this assembled audit trail allows an inquiry auditor to evaluate both the trustworthiness of my data and the meanings attached to them (Polit, et al., 2001).

\section{Transferability}

Lastly, transferability within a critical paradigm is dependant on my research context being described in enough detail, to enable the reader's evaluation of the study's importance to their own practise, research and theory development (Koch, 1996; Polit, et al., 2001). In accordance with a critical inquiry, however, the findings from this return-to-work study have been linked to Freire's critical social theory, rather than specifically generalising them to a population.

\section{Anticipatory accommodation.}

Denzin and Lincoln (1994) caution that making uncritical generalisations from one research study to another accepts a one dimensional cause and effect universe. Hence, their circumspect approach to making research generalisations is encapsulated within their third and last critical rigour requirement 'anticipatory accommodation'. This construct required me as the researcher to utilise my knowledge of a variety of comparable and different research contexts, to begin to process the similarities and differences to this study. This is because both the researcher and the reader need to make sure within transferability, that the contexts being compared are similar.

\section{Ethical Dimensions}

Beneficence, respect for human dignity and principles of justice are standardised research protocols, which have special significance when working with vulnerable populations (Polit et al., 2001). As this research involves mental health clients, ethical approval from the Ministry of Health, Regional Health and Disability Ethics Committee was required. Prior to being submitted, the completed ethics application was reviewed by the issuing committee for the DHB Māori letter of support, required by the Ethics Committee. The key issues related to ethical considerations are set out in the attached information sheet (Appendix A), which was fully discussed with the informants following formal ethical approval, and prior to them signing the consent form (Appendix B). 


\section{Beneficence}

Freedom from harm.

The research informants needed to be protected from potential physical, social, psychological and financial harm (Polit, et al., 2001). This meant being proactive in eliciting feedback and identifying procedures for the airing of informant concerns. Consequently, backup supports were made available and included counselling and liaison with pre-identified key workers if any of the informants were observed to be stressed or exhibiting early warning signs because of their participation in this study

\section{Freedom from exploitation.}

Involvement in this study should not subject the informants to any disadvantage, or expose them to situations they are not prepared for (Polit, et al., 2001). During the writing up of the report, particular attention was given to ensuring participation did not identify and thereby compromise the informants' employment, or prospective employment situations. In addition, as the relationship distance between the researcher and participants typically declines as the study progresses, I needed to attend to maintaining the integrity of ethical considerations and interpersonal relationships throughout the research. Potential boundary dilemma's such as interviewing clients at home in the evening and in the weekend were raised and reflected on during supervision and peer debriefing meetings.

\section{Risk/ benefit ratio.}

The degree of risk taken by the informants should never exceed the potential benefits of the knowledge to be gained for the group under study (Polit, et al., 2001). Thus, an important initial question in assessing the overall risk/ benefit ratio in this study was whether the study's focus on return-to-work experiences, had the potential to improve access to employment opportunities. The informants' informed, voluntary decision to participate was made on the basis of the participant information sheet (Appendix A) which made a full and detailed disclosure about the risks and benefits of their participation.

While there were no expected physical risks, psychologically there was the potential for people to experience distress while relating negative or traumatic 
memories related to their return-to-work experiences. Backup supports were made available to the informants. Prior to each interview, the informants and I identified a primary key worker who was willing and able to debrief with the informant if needed. Each informant also had the option to invite a support person to the interview, and three informants availed themselves of this option. As I am experienced in working with and supporting people who experience mental illness, I closely monitored the informants' reactions and mood during the interview process. On two occasions, I stopped and turned off the tape when the informants' conflict about what to disclosure was evident, resuming only after the issue was resolved. At the completion of each interview, I asked the informants whether any aspect of the interview had been unsettling. In addition, as prearranged, I also rang and checked in with each person the following day. While one person reported immediately after her interview that she felt 'emotionally stirred up', this quickly resolved (see journal note under the 'data collection' heading in this chapter p. 58). This was the only report from the informants or key workers of any measure of distress in relation to informants in this study.

Respect for Human Dignity

Right to self determination.

As the informants are members of a disenfranchised community, an important critical ethical requirement is to ensure the informants' right to self determination. This means that the informants had the right to decide voluntarily whether to participate in a study without any coercion. As documented in the participant information sheet (Appendix A), informants also had the right to seek information; refuse to give information and to terminate their participation. Polit et al. (2001) caution that offering a stipend may seem to be coercive, particularly when the group under study is considered vulnerable. The mental health consumer movement, however, strongly recommends remunerating people with mental illness when seeking their expertise. Hence, it was appropriate that I 
provided a koha ${ }^{11}$, in terms of vouchers and tokens of appreciation, plus reimburse any travel costs associated with the informants' participation.

Right to full disclosure.

This requirement determined that the informants' informed, voluntary decision was made on the basis of the participant information sheet (Appendix A), which made a full and detailed disclosure about the nature of the study, and my responsibilities as a researcher, including identifying the informants' rights, and the costs and benefits of their participation.

\section{Informed consent.}

Informed consent is based on self-determination and full disclosure described in the preceding paragraphs. It required that informants had adequate information regarding the research, comprehended the information and had the power of free choice, enabling them to consent voluntarily to participate in the research, by signing the consent form attached in 'Appendix B'. Informants were also informed that while their narratives may have positive benefits for the general population under study, their involvement may not directly benefit their own employment situation.

\section{Principle of Respect}

As the informants in this study are members of a disenfranchised community, an important critical ethical requirement was to ensure their right to selfdetermination by affording them influence and power as described in the methodology section of this proposal (Depoy \& Gitlin, 1998; Rogers \& PalmerErbs, 1994). The selected research tradition required me to operate within an open and honest context, at each stage of the research process and in the deployment of the findings. Embracing the key critical paradigm values of reciprocity, participation and power sharing means I relied on informant feedback throughout the study, and within the member check procedures (as described throughout the rigour section in this chapter).

\footnotetext{
${ }^{11}$ Koha is a Maori term for a gift which is given as a donation or expression of gratitude (Ryan, 1993).
} 


\section{Principle of Justice}

Right to fair treatment.

The informants had the right to fair and equitable treatment which required me to honour mutual agreements regarding practical arrangements and process. By adhering to the rigour procedures described earlier in this chapter, I was able to ensure that the informants' contributions were treated justly and professionally. This included me ensuring their right to access professional help if desired; the right to not participate, or withdraw without prejudice, and the right to clarify information at any point in the study.

\section{Right to privacy.}

Confidentially is of the utmost importance when working with this population under study. Hence, it was imperative that the demographic and workplace information, and quoted dialogue within this study have no identifiable features. Pseudonyms have been used and the workplaces have been referred to in expansive terms such as the food services industry, trade position. After consultation with the Māori cultural advisor who provided oversight to this study, it was decided to use the term Kaupapa Māori service to describe a worksite as the informant, Louis, had requested. These confidentially procedures have been subjected to double checking within supervision. Informants also had the opportunity to remove information from their interview transcript before it was analysed. It was stipulated to informants, however, that if they disclosed that they or any other person was being harmed, or there is significant potential for harm, that that information would be shared with a responsible clinician. Prior to interview, each informant was informed that all their information would be gathered, collated and stored within a regime that operated with the strictest of confidence. Hence interviews took place in a private place, the transcribers confidentially agreement was explained and signed (Appendix D), and the transcribed data will be stored electronically (on disc) for ten years in a secure location, and then destroyed by incineration. The informants have been offered their audiotapes; those which remain unclaimed at the completion of this study will be destroyed. 


\section{Te Tiriti o Waitangi}

The three core constructs which underpin the Treaty: protection; partnership and participation, determine our obligations as health researchers, to the three articles documented within the treaty. While the Treaty has implications for every health research study, because four of the informants were Māori, these implications have heightened ethical and political significance in this study. As discussed in the literature review (see p. 30) Māori are over-represented within both forensic admission and unemployment statistics. The following paragraphs detail the researcher obligations when both conducting this study and reporting the findings.

Under article one, as a health practitioner and researcher, I was obliged to offer kawanatanga or governorship to Māori, in respect of Māori who are research informants, and the cultural advisors who represent them (Chaplow, 1993; Durie, 2001). Staff from the forensic psychiatry Kaupapa Māori unit provided me with mentoring to ensure the tangata whaiora's cultural values and beliefs found safe expression during this study. In accordance with article two, health practitioners are required to assist Māori to retain their rangatiratanga of taonga; everything that is held precious (Chaplow et al., 1993; Durie, 2001). During this study, a Māori cultural advisor was consulted about the informants' interviews, whanau consent (Appendix C) was sought to accompany informed consent and both cultural and whanau representation at interviews was encouraged. In addition, the informants have been offered their audio-tapes, with acknowledgment that their shared stories are held as taonga.

Under article three the Crown agrees that the faiths of Maori custom should be protected (Durie, 2001). This premise determines the identification of those traditional customs and tikanga which are appropriate in health, and then protects their competent and appropriate usage. A core understanding that underpins all New Zealand health is that Māori cultural conventions, such as those related to a secure Maori identity, are associated with health. (Chaplow, et al., 1993; Durie, 1998; Jungersen, 2002; National Health Committee, 1998). Article three also requires the crown to offer Māori the same rights and privileges as British 
subjects. Hence, Māori have the mandated right to equitable access to any Crown-funded resource (Durie, 2001; Christie, 1997). Durie (1998) describes the reasons behind the statistical trend towards Māori being over-represented in inpatient mental health settings, as illustrated within forensic psychiatry statistics, as being diverse and complex. During the research process and when reporting the findings, I need to be mindful of these socio-political factors, particularly in terms of the historically constituted positions related to cultural oppression and the consequential impact on psychiatric hospitalisation and unemployment statistics.

\section{Chapter Summary}

Consistent with a critical hermeneutic methodology, the initial design was receptive to the research procedures being refined in response to the emergent process. In line with the critical paradigm values of reciprocity, participation and power sharing, I relied on informant feedback, and the supervision and peer debriefing processes to plan and implement the dynamic processes of data collection and analysis. The research method and design, which is underpinned by critical hermeneutic methodology, posed a considered and congruent research framework. This framework ensured internal consistency; an overall vision and a thorough in-depth analysis of the informants' return-to-work experience. 


\section{CHAPTER FIVE: THE LIBERATORY QUEST: FROM ALIENATION TO INCLUSION AND EMANCIPATION}

\section{Chapter Overview}

This is the first of three chapters that seeks to reveal the understandings which have emerged from the data. In this chapter, the first four overarching themes: 're-entering the world'; 'fortitude'; 'redeeming oneself' and 'being set apart' are all concerned with the informants' liberatory quest. These overarching themes illuminate that returning to work was a situation that held creative possibilities for all the informants. It was therefore a redeeming opportunity to prove themselves under the scrutiny of colleagues, whanau ${ }^{12}$ and the wider community, rather than just the secluded and marginalised world of psychiatric care.

As this is a critical study, I needed to organise the findings in order to make the thematic understandings accessible to the informants. Therefore, each of the overarching themes is further broken down into themes that seek to represent aspects of the informants' return-to-work experience. This process has resulted in more phenomenological themes than may be expected in a Masters thesis. Presenting the findings within discrete experiential thematic constructs, however, enabled the themes to be both accessible and meaningful to the informants, which is an important critical consideration. These experiential themes are represented by titles that consist of meaningful words or phrases, which are seen by the informants as being plausible aspects of their return-to-work experience. In accordance with a critical design, the knowledge that emerged from the themes sought to imbue the informants with an increased awareness of their own return-to-work experience, as well as their collective socio-political interests (Burns \& Grove, 1997). This chapter's four overarching themes, and the themes that contribute towards the experiences conveyed in each overarching theme, are presented in the following summary.

\footnotetext{
${ }^{12}$ Whanau is the Maori term for family (Ryan, 1993).
} 
Chapter Five: Themes

Re-entering the World

Reinventing oneself.

Revitalising work ethos.

Getting your life back.

Turning your life around.

Fortitude

Persevering against the odds.

Navigating through the complexities of disclosure.

Dealing with unwanted disclosure.

Being bolstered by others.

Being Set Apart

Fear of being exposed.

Being alienated from others.

Keeping colleagues at arms length.

Being scrutinised and judged on the basis of diagnosis.

Being teased and bullied.

Keeping safe: Staying right away from drugs \& alcohol.

Redeeming Oneself

Re-establishing family pride.

Others believing in you vis-à-vis believing in yourself.

Proving oneself.

Spirituality resonating at work.

In applying the critical lens to the informants' experiences, each of the four overarching themes is concluded by a brief summary which includes the Freirean perspective. As an orientation to this chapter, the Freirean notions that are used within these four overarching themes, previously described in detail within the methodology section (see p. 43), are again briefly outlined below.

\section{Authentic Reflection}

Authentic reflection is critical reflection that is grounded in the here and now, and involves the situation in which disenfranchised individuals find themselves submerged (Crotty, 1998; Freire, 1972; Heaney, 1995; Smith, 2002).

\section{Transformative Action}

Men and women are called to transformative action in order to transform the human world, and thereby transform themselves. Hence, transformative action involves critical refection on the reality of ones own situation, in order to meld 
the reflection/ action cycle of praxis (Crotty, 1998; Freire, 1972; Heaney, 1995; Smith, 2002).

\section{Humanisation}

Humanisation is a term that encapsulates the quest to be more fully human by creating a humanly enhancing culture as a fundamental means of striving for liberation. The process of humanisation strives to liberate both the oppressed and the oppressors (Crotty, 1998; Freire, 1972; Heaney, 1995; Smith, 2002).

\section{Praxis}

Praxis is a complex cyclical emancipatory process of action- reflection-action upon the world in order to transform it. As the action and reflection components are interdependent, praxis cannot be divided into a prior stage of reflection and subsequent stage of action. Thus, when action and reflection are united they create culture and society by constantly and mutually illuminating each other (Crotty, 1998; Freire, 1972; Heaney, 1995; Smith, 2002).

\section{Conscientisation}

Freire links conscientisation to the relationship between humans and their world, and to praxis. Conscientisation is a process that involves identifying contradictions in experience through mutual dialogue. This ultimately leads people to break through prevailing societal myths to reach new levels of awareness, and in particular an awareness of oppression (Crotty, 1998; Freire, 1972; Heaney, 1995; Smith, 2002).

\section{Critical Consciousness}

According to Freire, critical consciousness is characterised by an in-depth interpretation of problems. This level of mature critique occurs through testing one's own findings, while remaining receptive to further revision. Inherent in striving for critical consciousness, is the rejection of passive compliance in order to embrace collaborative and dialogical processes (Crotty, 1998; Freire, 1972; Heaney, 1995; Smith, 2002).

\section{Dialogical Method}

The only valid approach to liberation, Freire believes, is the way of mutual dialogue. Without dialogue, there is no communication, and with no 
communication there can be no liberation. Hence, this egalitarian dialogical process requires committed involvement by all concerned parties (Crotty, 1998; Freire, 1972; Heaney, 1995; Smith, 2002).

\section{Problematisation}

Freire's problematisation is a pedagogical process that sets out to engage the affected group in the task of translating the situational reality into meaningful symbols, which are then able to generate critical consciousness. In particular, attention is given to the interrelationship that these problems have with both nature and oppressive social forces. In this regard, problematisation has a parallel process to demystification; with Freire stressing the need to problem pose the myths handed down to society by the oppressors. Problem posing is an initial task within the problematisation process, which allows all previous conceptualisations of a problem to be brought into question (Crotty, 1998; Freire, 1972; Heaney, 1995; Smith, 2002).

\section{The Culture of Silence}

It is uniquely difficult for people who are disenfranchised to become critically aware of their marginalised position, and intervene in their reality, because they belong to the culture of silence. Freire uses this term because, in their situation of marginalisation, people have no political voice. Hence, the disenfranchised are effectively silenced, and therefore, they feel incapable of being self-determining (Crotty, 1998; Freire, 1972; Heaney, 1995; Smith, 2002).

\section{Liberatory Action}

Liberatory action seeks to transform the social order by promoting participation and participatory structures, while denouncing authoritarian and alienating systems of organisation. Hence, the governance of liberatory action is dialogical and egalitarian, as personal freedom and the growth of individuals can only occur in mutuality with others (Crotty, 1998; Freire, 1972; Heaney, 1995; Smith, 2002).

\section{Alienation}

Freire's theories are led by a concern for the oppressed. These are the people who have been historically and culturally alienated within a regime of oppression 
imposed by those in power. Alienation was originally a Marxist term, and refers to the subjection of the people by the social elite, resource constraints and the political forces of hegemony (Crotty, 1998; Freire, 1972; Heaney, 1995; Smith, 2002).

\section{Summary}

According to the underpinning tenant of the Freirean (1972) constructs outlined in this introduction, the task of the disenfranchised is to liberate themselves and, in the process, liberate society from their prejudicial attitudes. Within this chapter, these constructs are used to illuminate the informants' experiences of their return-to-work. They are notions, therefore, that help define the informants' liberatory quest from social alienation in a secure care hospital, to the social inclusion associated with being a worker.

\section{Overarching Theme: Re-entering the World}

Re-entering the world of work was seen by all the informants as a defining moment within their recovery journey. Joe and Lavina commenced their open employment jobs while they were still residing in a forensic inpatient setting. All the other informants' secured employment post discharge; a feat everyone accomplished within six months of leaving the forensic hospital. The following four themes: 'reinventing oneself'; 'revitalising your work ethos'; 'getting your life back' and 'turning your life around', represent the informant's experience of navigating through the rewards and the complexities of re-entering employment, after years of exclusion from the world of work. From a critical perspective, these experiences are explored in terms of the Freirean (1972) concepts of authentic reflection and praxis, which lead to the more sophisticated activities of transformative action and conscientisation.

\section{Reinventing Oneself}

Within Forensic Psychiatry, both the clients and their practitioners are required to balance the frequently contradictory goals of social inclusion, treatment and the public's perception of safety (Couldrick \& Aldred, 2003). These contradictions are brought sharply into focus with any moves made towards the clients' 
community reintegration. These ambiguities are reflected in Maria's following critique about the process of reinventing herself. Within this theme, the informants' experiences of reinventing themselves are used to illuminate Freire's notion of a liberatory quest, a journey that occurs in and through the processes of authentic reflection, praxis and transformative action.

\begin{abstract}
I knew I couldn't go back to the job I was trained for..//. so that was taken away from me and it was like reinventing myself. Well it was like "what can I do, who am I". You know that [profession] was such a large part of how I identified myself, so here I was, not only was I re-entering the workforce after many years in a very unusual situation but I also felt "what can I do then". Going back to work was a worry, and yet I had a work ethic. I had a belief that I should be working because I knew I was capable of that. I also felt that I should support myself and there was a part of me that wanted to do it as well, because I wanted the stimulation and the interest of being employed somewhere. (Maria, p. 4)
\end{abstract}

Maria is critically reflecting on her essential relationship with the return-to-work phenomenon, and in doing so, her self critique illuminates the process Freire (1972) describes as authentic reflection. Rather than dwelling on the abstract, authentic reflection occurs within the material and human circumstances of the here and now. Therefore, it is reflection that is concerned with the actual situation that the person finds themselves ensconced. Authentic reflection then becomes the instigator for praxis. As exemplified in Louis' following excerpt, the action and reflection cycle of praxis both arises from, and is driven by, his active intervention in the historical reality of his mental illness, illness-related offending and secure care hospitalisation.

I think it's being through the system; you know it makes you humble; you've got more affinity with others. When you've been at the bottom for a long time, you know you can only go so far backwards. There's only one other way to go in either direction, it's like going back up, or hang around the bottom for the rest of your life, yeah ...//... don't get bogged down on the past, the past is the past, yesterday is history, tomorrow is a mystery, so let's do it today. (Louis, p. 17)

Within the above passage, Louis is illustrating Freire's (1972) transformative action that arises from engaging in the action and reflection cycle of praxis. Louis' critical insertion into the reality of his own situation, called him to 
transform his world, and thereby transform himself. Thus, the informants' experiences that are reveal the essence of the liberatory quest. Both Maria and Louis are authentically reflecting on how they chose to engage, and have continued to engage, in reinventing themselves beyond the confines of their former institutionalised reality.

\section{Revitalising Work Ethos}

Maria and Rod, highlighted the fundamental importance of revitalising their own personal work ethos, and placed this fact within a context of having a later onset of their illness compared with many of their compatriots within the forensic system.

Well, let's say the life experiences were just my background, like working from the age of fifteen right through: fencing; scrub cutting; all the hard jobs and just your natural ability to work. (Rod, p. 7)

Even though my work had been interrupted for five years, I had been luckier than perhaps some people in that I got unwell when I was older, so I already had a work ethic in life, and I knew what the expectations were of work. (Maria, p. 8)

Maria and Rod's previous work competencies exist within the context of an enduring work ethic. They both perceive that acquiring a work ethos early in their career has given them an advantage over many of their former fellowresidents in secure care. They see their work ethic as a means of successfully renegotiating their re-entry to work.

For all the men in this study, their experience of revitalising their work ethos bore a strong relationship to their childhood view of their fathers' work. The male informants all placed their return-to-work in terms of a historical family work ethic. While the men acknowledged that health and vocational professionals had played a role in enabling their return-to-work, it was their fathers who laid the initial foundation upon which they had built their work ethos.

Probably not anybody in the organisation, it was probably my father. My father became disabled at a young age, but he went to 
work everyday. He might go out and have few beers and come home drunk but he still got up and went to work. (Louis, p. 8)

Dad prepared me for work, and like the forensic rehab staff pushed me to go back to work. (Joe, p. 17)

According to the all the male informants, this foundation had been established during their formative years of early adolescence, as they both observed and worked alongside their fathers. In contrast, while all the women informants acknowledged their family's support for their current work goals, they did not describe learning their existing work ethos within their family or whanau context. Instead, the women saw that their work ethos had evolved in response to their successes in both education and work settings. The different ways in which the men and women in this study had established their work ethos and skills, was the most distinct gender difference identified during the analysis. This finding serves to provide a poignant insight into the political and historical influences on gender work roles, which are now well acknowledged within current New Zealand employment literature (Mintrom \& True, 2004). The breadth and complexity of this historical reality precludes further examination within this text, although the profoundly influencing nature of gender work roles, both historical and present, invites further dedicated research from a disability and work perspective.

\section{Getting Your Life Back}

'Getting your life back' is the term which I heard used most often to describe the informants' experience of returning to work after the catastrophic devastation caused by their illness and offending. This devastation resulted in many losses. The loss of confidence, and self and social esteem, became a reference point for many of the informants as they talked about their work place gains. "Getting my confidence back and meeting new people, that sort of thing, yeah, it was one helpful experience" (Terri, p. 1). Freda talked in terms of what she was both gaining and leaving behind in relation to her new found autonomy: "Going back to work and living, no staff telling you what to do and what not to do" (Freda, p.14). In delving further into the concept of weighing up the costs and benefits of change, Maria describes discerning which dormant life skills she needs to 
revitalise, and which adaptive behaviours she needs to leave behind in her former institutionalised world.

As you develop your work skills again and you develop over time, because being in an institution for five and half years is a long time and you lose a lot of your daily living skills, and you develop skills that help you to survive in that environment so you have to let those go, and you have to gradually relearn how to be in the world. (Maria, p. 19)

Maria is reflecting on her life world as being a culturally, historically and politically constituted existence. Her authentic reflections on the shifts in power that accompanied her move back to the community enabled Maria to take action and retire her institution-driven survival skills. This move has bolstered her success within her new self determining life world. As Maria's account continues, she focuses on the experience of regaining positive elements of her previous life that she had feared may have been lost to her forever.

So I started work with nothing really, but what I did have, and that probably prompted me along, was that I wanted to re-enter the world that my family and friends were living in and so that probably helped to push me along as well, so yeah, I wanted a life back again really, and this was a way of working towards that. (Maria, p. 15)

In taking an active role in transforming her situation of secure care hospitalisation and unemployment, Maria has shaped the very conditions for her existence. This echoes Freire's (1972) belief that the call of human beings to creative initiative extends to their own being. Returning to work significantly enhanced the informant's experience of getting their life back after their years of isolation in a secure care facility; illuminating Freire's notion that human freedom ultimately means being self creating.

\section{Turning Your Life Around}

All the informants spoke about healing from the catastrophic impact of their illness during their time in a Forensic hospital, and then recapturing their former skills and resources in order to get their life back on track. For Louis, Kiri and Joe, however, their healing encompassed a further dimension. They regarded their time in the secure care hospital as being the catalyst in turning their life 
around. Their retrospective view is that they were leading a life that was in a downward spiral due to the combination of substance abuse and 'risky' associates.

When you're always in trouble with police, or getting in trouble with gangs, just getting into trouble, and not really living a life it's just making trouble, but then I had the mental illness dealt with at Forensics. Just give yourself time, everybody, you get there in the end, I mean I waited to get better for years, but I got there in the end, a big turn around. (Kiri, p. 14)

Just wanted to get my life in order and words are cheap, actions speak louder than words, everybody knows that, and I was also a drug addict and a bad one at that, so yeah. I just wanted to get my life together really, and now I'm a role model for my daughter. (Louis p. 8)

Kiri and Louis' excerpts illustrate Freire's (1972) belief that inherent in being human, is the call to be self-creating. In line with this thinking, the informants self-creating can be viewed in terms of Freire's notion of conscientisation. During their lengthy stay in a secure care facility, both Kiri and Louis were able to emerge from their former lives which involved gangs and substance abuse. They were then able to reflect upon their situation, and intervene in it. Through such transformative action, Freire (2004) purports, people cease to see their situation as a blind alley. Kiri, Louis and Joe were able to look ahead and move forward with a new found spirit of courage and hope.

\section{Summary}

For people who experience mental illness and have a history of prior illnessrelated offending, securing employment accentuates their 'recovery' outcomes, as this is the juncture where they are seen to rejoin society. The role shift from sickness beneficiary to worker was integral to the informants' experience of reinventing themselves. Assuming a worker status also elevated the informants to a position that enabled them to see beyond their historically limited horizons, and thereby regard themselves as being central to determining their own destiny. This liberatory quest towards self determination both supports, and is supported by, Freire's (1972) notion of conscientisation. According to this construct, to be human is to be involved in the never-ending project of being self-creating. 
Therefore, striving for conscientisation required the informants to engage in transformative action in order to revitalise their work ethos and turn their lives around. For both the person and their family or whanau, being in a worker role underscores the individual's wellness, and serves to create a perception of distance from the devastation created by the illness and prior illness-related offending.

\section{Overarching Theme: Fortitude}

Fortitude is the overarching theme that encompasses the experiences which received the most comment from the informants. Accordingly, the informants' fortitude is exemplified in both the challenges and triumphs which are highlighted in the themes: 'persevering against the odds'; 'navigating the complexities of disclosure' and 'dealing with unwanted disclosure'. The last theme, 'being bolstered by others', unifies all the other themes as Freire's (1972) notion of liberatory action being a joint project is illuminated through the informants' narratives. The informants' experience of fortitude is related to the Freirean concepts of problematisation, demystification, praxis and conscientisation. These are liberatory action processes, which through mutual dialogue and solidarity, lead to critical consciousness.

\section{Persevering Against the Odds}

The informants identified that persevering against the odds was a common experience. From the initial contemplation of possible work options, all except one informant (Kiri) described the process of seeking and securing employment as being a daunting prospect.

../.. every morning I'd look at the Herald and I felt very alone, I felt like, "I can't handle this" eventually it came to that point and I thought, "I don't know how to do this, this is too big for me", so I think that's probably a realistic issue for people coming out of my situation that it is the enormity of it and where the hell do you start and your life has been so interrupted in the sense that what are you supposed to tell an employer. So eventually what happened is I looked and I looked and I looked. (Maria, p. 4) 
Well yeah, I always thought to myself I'd like to get a job but I didn't know whether I would be able to, or could handle one, I just thought I'd try and get through each day. (Louis, p. 9)

Louis is identifying that his ability to overcome the odds was enhanced by breaking down the larger more daunting goal of open employment into shortterm achievable tasks. In the following excerpt, he is describing how the skill of goal setting can move an individual from a position of non-achievement and low expectation, to securing employment: "Oh you know, "don't worry about that one mate; you know you will never get there", and then they teach you how to put steps together; putting in those little steps (Louis, p.16). Focusing on his recovery goal of work was made possible by seeing each step on the way, as a day by day achievement.

All the informants acknowledged that returning to work was a defining moment in their recovery. This shift to being a worker brought home to the informants what was required of them, and their support people, as they faced the world again after their segregated and insulated years of societal separation.
../.. when you come out of somewhere like Forensic Services you do need a second chance and you have to find people that will give you a second chance, dealing with what's happened. I can understand how some people become more debilitated or unable to enter back into ordinary day life because they haven't had some of those supports, or sometimes I think we make choices as well, you know, you have to push yourself, everyday you have to push yourself. (Maria, p.13)

Maria required both emotional and physical fortitude as this experience of pushing herself out of bed every morning did not abate over time. Joe also described requiring ongoing fortitude in order to manage the more physically challenging tasks which were part of his daily work assignment: "Yeah, it was hard yakka ./l.. but someone has to do it. You gotta do it, there's no way about it, you can't run away from it 'cause it's still going to be there” (Joe, p.6). For Maria, Joe and Lavina, their need to physically and emotionally push themselves during their workday was enduring. While the other informants also lacked work stamina initially, the feeling of being depleted by their workday dissipated over time. As Kiri describes, the building of work stamina immersed her in the reflection/ action cycle of praxis. 
Just working really hard for the first boss that I had and then once you've got them under your buckle, everything starts flowing in. It's not one of those things that people just do; you have to work at it. You have to work at it before you can get any rewards. (Kiri, p.13)

In accordance with Freirean (1972) thinking, the informants who engaged in a consciously critical confrontation with their problems, as Kiri illustrates above, employed a problem posing approach. Therefore, other solutions became available to them as they stepped outside the limitations imposed by societal myths about mental illness. Such myths include that mental illness always correlates with work ineptitude (Anthony et al., 2002). All the informants described needing to persevere and find workable solutions, both alone and in collaboration with others, to overcome considerable employment-related odds. These impediments were concerned with aspects of self and illness, as well as those external barriers arising from societal myths and stigma about mental illness.

\section{Navigating through the Complexities of Disclosure}

Within the employment context, the issues of disclosure are inherently complex and are therefore often the topic of contentious debate within the mental health employment sector. While legislative guidance is provided by the Privacy Act (1998) and the subsequent Privacy Act amendments (Privacy Commissioner, 2003), there still continues to be considerable debate within the mental health vocational arena about the issues of disclosure. As this study is focused on lived experience, rather than practitioner guidelines for practise, this dilemma is explored from the perspective of people who are affected by mental illness.

It was evident throughout the informants' stories, that like vocational practitioners, they had all carefully considered the complexities related to the issue of disclosure from the time they started to contemplate re-entering the workforce.

The thought of actually ringing up an employer was quite frightening because I'd think well what am I going to tell them, they are going to ask me, "what have you been doing for the last five and half?" And what do I do? (Maria, p.1) 
The informants' reported that the legitimacy of their initial anxieties about disclosure was well acknowledged by their mental health and vocational practitioners. Therefore, as demonstrated in Maria's following account of her conversation with a career advisor, most of the informants described that disclosure had been addressed within situations such as practitioner-led job clubs and one-on-one rehearsal sessions with practitioners.

I think I even told her that I had a forensic background, I'm not sure, I might have just told her that I had a mental illness and she said to me "Maria, don't worry too much about that because you don't present like that at all, you don't look like you're still trying to live with your mental illness", so that was actually quite reassuring for me to get a professional tell me that. (Maria, p. 2)

In addition to these rehearsals, the informants all provided rich narrative about their actual experiences of managing both planned and unplanned disclosure, moving the dilemma from the theoretical to the actual. In the following excerpts, three of the informants express opposing views, about disclosing prior to being appointed to a position. Kiri has strongly held views about the need to withhold information, in order to initially secure a position.

So you know, you can't tell people, "look I've got a mental illness and this is what I did, this is where I've been" because you can't, 'cause people won't accept you for who you are unless you get them to get to know you first. Then you tell them, "look I've got a mental illness", but then you don't tell them everything. You don't tell them the crime you committed when you were unwell; you don't tell them about that 'cause then you don't get a job, that's the way it goes. (Kiri, p. 4-5)

For Joe, however, colleagues knowing his mental illness prior to him returning to work eased his job commencement and provided him with workplace security.

All the guys on the job working for the boss, they all knew me and knew all my sisters, so they knew my history and it was easier to relate to them because they already knew it and they knew not to give me dope on my job 'cause they know what it did to me. (Joe, p. 7-8)

By his workmates knowing him as a person, and knowing his whanau, Joe felt assured that they will look beyond his mental illness and his illness-related offending, to see him as a valued colleague. Knowing about Joe's mental illness 
also enabled his colleagues to take positive steps to ensure the workplace was physically and psychologically safe for him.

Louis has a view that complements Joe's experience. Louis' perspective is based on his experience of being employed in a Kaupapa Maori service. He perceives that the underpinning tenants of this service positively influenced his employment (as described in the next theme see p. 81). By disclosing fully, Louis believes that this will assist in securing both the right job, and the workplace accommodations and employer understanding that may be required to employ a wellness-at-work approach.

I think being up front is a good thing to do, because if you are not then you're too tired or you're unwell or you got a doctor's appointment to see a psychologist they're going to wonder why you're taking all this time off, whereas if you tell them up front they'll know. And if they are not happy with that, well then maybe that's not the job. (Louis, p. 3)

Both Joe and Louis felt safer that their background was known by their work colleagues. They describe that this collective collegial knowledge led to them to being accepted, thereby creating a supportive work environment that was ultimately more enabling of their work performance.

Maria also valued having select senior managers know about her history prior to her employment with a large corporation. Because someone in senior management was aware of her situation, Maria felt protected in the event of her history being exposed, as had previously occurred.

../..what I felt very excited about was that you kind of have this idea that if a manager of an organisation is just a kind of figures person, he's not particularly a people person. But he must have had a very kind heart because he said, "no that's fine as long as she can do the job that's fine, we'll work through that". (Maria, p.5)

It added to Maria's sense of security and aligned with her sense of moral obligation to ensure that this senior manager was fully informed of her history. She was more circumspect, however, about disclosing to other colleagues. As Maria portrays in the next excerpt, she was very discerning about the personal information she gave to the different management tiers above her. 
There was a woman, who was my own manager, and then there was a woman above her and they both seemed really nice and friendly but I decided just to say that I had been unwell for a time. I didn't say what with, so my own immediate boss never knew that I had a mental health issue but the one above her did know that I had a mental health issue. I don't think she knew I had a forensic issue, but she knew I had a mental health issue. So, I felt like they accepted me. (Maria, p.6)

Although the informants' disclosure occurred at different times, varying from pre-employment to two months later, eventually all of the informants did inform at least one person in a management role about their mental illness, with five people stating their employers also knew the details of their mental illnessrelated offending. In every work situation that was explored in-depth within this study, the informants had received a supportive and compassionate response from the boss(es) they had selected to disclose to.

As previously discussed in this theme, Louis and Joe were the only informants who were aware and comfortable that all their immediate work colleagues knew their history. As Maria exemplifies in the following excerpt, all the other informants were more circumspect about colleagues knowing about their history.

So I continued on at work and got to know a few people. The person that I got closest to, and he still is a very good friend, and I still haven't told him about my situation. I had that dilemma, it became a big dilemma for me, I kept worrying about it ..//.. It wasn't a methodical kind of like, oh my god. It wasn't exactly totally that I can't talk to him about .... There was that recognition that probably of the people in the organisation, he wasn't a good person to talk with at that stage, I couldn't trust him with it. (Maria, p. 7)

Maria's self-reflective process mirrors Freire's (1972) conscientisation construct. This notion involves people being able to identify contradictions in their life world experiences, as occurred in Maria's experience of disclosure, and then through critical reflection and dialogue, this awareness eventually becomes emancipatory knowledge. All the informants provided very considered accounts that highlighted the complexities of disclosure. For most of the informants, disclosure was the dilemma that caused the greatest return-to-work angst. Although the informants' views differed considerably about to whom, when and what you disclose, they all concurred about the need to be circumspect. 


\section{Dealing with Unwanted Disclosure}

As the narratives portray, the fear of having their history of offending exposed at work was regarded as a major issue for all the informants except Louis. He attributed his openness, and his colleagues' acceptance, to being part of the work ethos within the Kaupapa Maori service where he is employed: "The organisation is a Maori Kaupapa, so it's not a big organisation..//. we are really close people, and easy to talk to" (Louis, p.7). For the other informants, however, this issue heavily influenced their circumspect approach to selfdisclosure in the workplace. Despite this circumspection, six of the informants did experience having their history of illness related offending unexpectedly exposed at work. In Joe's situation, his overall boss quickly dealt with a new colleague's gossip about Joe's illness-related offending.

The boss told him off and said, "aye don't go gossiping about my workers to them. They don't need to know any of that. You don't need to tell anyone of them either," and then the guy shut up then..//.. So the boss stuck up for me a few times. That's pretty cool. (Joe, p.11)

Joe's chagrin and embarrassment about the disclosure was accompanied by a feeling of being supported and encouraged by his boss' response. When Lavina inadvertently disclosed her history to her prospective employer, she also experienced both reassurance and acceptance.

../..he goes, "by the way how come you have only been working part-time" ugh. I was like backed into a corner and it just popped out because it was so unexpected. I didn't think he would say anything about part-time work and I just went, "oh, because of my parole status" (laugh) and he goes "it wasn't anything to do with stealing was it" and I was like, "no" and he goes, "oh that's fine then" (laugh). So he was the type of guy because he had already had someone from Mental Health he wasn't judgemental about people like us. He was just like, "was it about stealing?". And then I found out later that someone was stealing from him, so that's why he thought that if it wasn't for stealing, that's okay (laugh). (Lavina, p. 8)

For Maria, the divulging of sensitive information about her past arose from a source outside of her workplace. The public nature of this exposure, however, caused Maria apprehension about returning to work as she describes in the following excerpt. 
To push myself to go back to work was really hard but I knew I had to do it so I did and fortunately either if someone had heard it, they hadn't made the connection with me..//.. so I don't really know ... and I certainly never made any enquiries (laugh). (Maria, p. 9)

For five of the six informants who experienced unwanted disclosure, their initial angst about having their history divulged was actually ameliorated by the positive reaction, or in Maria's case the absence of reaction, from their colleagues. In contrast, in Rod's situation being recognised by a new colleague who'd previously known him, led to three difficult months of alienation from his colleagues.

There was a time there when a girl who used to know me before, she was working in the team and then it got back to me that they changed. Their attitude changed towards me and I knew she had been talking. It took three months to get through, I just had to, oh... it was hard. (Rod, p. 8-9)

Having their prior history of illness-related offending exposed caused considerable angst for the informants who experienced this realisation of their major return-to-work fear. For most of the informants, however, the positive reaction of others in their workplace actually served to ameliorate their distress. While Rod's experience of being 'outed' at work caused three hard months of feeling pressured and alienated, he believes his perseverance paid off and, "I felt like running then but now it's all sweet" (Rod, p. 9). As this nagging fear of being exposed at work abated, the informants experienced demystification. With demystification, a concept that Freire (1972) aligns with his problematisation construct, comes a new view of reality and a founded hope for freedom. As portrayed in this theme, in over eighty percent of the situations, the process of disclosure, both planned and inadvertent, led to the informants and their bosses "encountering one another in the common search to be more human" (Freire, 1972, p. 156).

\section{Being Bolstered by Others}

The 'Like Minds; Like Mine' anti-stigmatisation campaign seeks to address the stigmatisation of people with mental illness in New Zealand society. People affected by mental illness, including some high profile New Zealanders, describe 
their experiences and achievements within the media. Using an advertisement type in the media means that these moving narratives are repeated frequently in order to gain viewer attention. Recent campaign evaluations have indicted positive attitudinal shifts from employers. From the perspective of a person with mental illness, Kiri provides an account of how this campaign has also positively influenced her own self-concept and bolstered her personal work aspirations.

Look at what John Kirwin was, an All Black, and he had a mental illness. What about that Denise, the clothing designer../l.. It gave me like confidence to get out there and, I got a mental illness but that's not going to set me back. I can do anything after watching them, I can be anyone or anything, and here I am today after five years of experience I am finally being trained to be a supervisor. (Kiri, p. 6)

Affirmative public response to publicity campaigns, such as the 'Like Minds; Like Mine project', serve as a positive indicator of the increasing political network of supportive people who will reliably promote the collective and individual needs of people who experience mental illness.

From relying on others to facilitate the transformative action steps required to secure and retain employment, Louis describes in the following excerpt how he has now moved to become a facilitator of other mental health consumers' transformative action.

Well, that's all part of role modelling for others. Say like just being here and seeing some of the friends from back in the old day at Forensic, they might aspire to open employment one day, maybe not, but that's their decision. But I've also got no problems on how to bring people to that point, or them asking me questions on how did you get there, how did you get to the job, you know, as well telling them it's hard work, you know, reliability. (Louis, p. 6)

Louis' narrative portrays Freire's (1972) thinking about how liberatory action occurs within a joint project, and as such is reliant on mutual dialogue. This unified action, therefore, leads to people being bolstered by others in order to overcome adversities and reach their potential. In concluding this theme, these emancipatory outcomes are illustrated within Kiri's reflections on her relationship with her understanding boss. 
Just people that understand, you get people who don't understand mental illness, people that can't, people who seem to have their own judgement about it, but they don't really understand it. But no, my having an understanding boss was the most amazing thing 'cause it makes life so much easier, and it makes you feel really good about yourself and that you can achieve anything when you feel like that. (Kiri, p. 4)

\section{Summary}

Freire (1972) purports that as individuals continue to modify their environment through human activity, as occurred for the informants when they returned to work, ever new challenges and tasks will arise. The informants' fortitude is explored within the context of the first three themes, which are concerned with persevering against the odds and the complexities related to the controversial issue of disclosure. When, what and to whom to disclose is regarded by most of the informants as the most challenging issue facing people with mental illness who are entering the open workforce. Within the mental health sector, it is arguably the vocational practise issue that engenders the most debate among practitioners due to the complexities involved. Accordingly, the fourth and final sub theme, 'being bolstered by others', highlights the Freirean notion that people who are marginalised within society, need help to emerge and engage in the struggle for social inclusion. Therefore, being bolstered by others significantly enhanced the informants' fortitude. Accordingly, the Freirean thinking that liberatory action can only exist within a joint project, is evident in many of the informants' return-to-work reflections.

\section{Overarching Theme: Being Set Apart}

Many people who are affected by mental illness face exclusionary practises due to the impact of stigma and discrimination. The principles of social inclusion recognise that people who have restricted decision-making rights and limited access to the work, education and social opportunities that affect their lives are denied citizenship rights (Freire, 1972; Joe, 2003). As members of a disenfranchised group, it is not surprising that all the informants in this study spoke about their lived experience of being set apart in the workplace, although the degree and the extent to which this was realised varied considerably. As the 
range of informants' experiences are explored within the context of the six following themes: 'fear of being exposed'; 'being alienated by others'; 'keeping colleagues at arms length'; 'being scrutinised and judged on the basis of diagnosis'; 'being teased and bullied' and 'keeping safe: staying right away from drugs and alcohol', they are critiqued in relation to the Freirean (1972) notion of 'the culture of silence'. As highlighted throughout the following themes, in order to emerge from this culture of silence, people need to employ the emancipatory processes of liberatory action, problematisation, demystification, conscientisation and the dialogical process.

\section{Fear of Being Exposed}

All the informants in the study had at least one person in authority at their place of work, who knew about their mental illness and prior illness-related offending. Six of the informants, however, spoke about their fear of having their history, particularly related to their illness-related offence, exposed within the work situation. As Maria describes in the following excerpt, her fear of being exposed extended back to when re-entry to work was first being seriously considered, resurfacing at significant times in the employment process.

So they accepted me and so I got through and found myself employed at the organisation (laugh). But it was always a bit nerve-racking; I kept thinking who's going to find out about me, what if they recognise me, all of that kind of stuff. And also it was good in the sense of learning the art of superficial conversation again, because that's all I could have mostly and yet somehow find a way in. (Maria, p. 6)

The dilemma of establishing connections with work colleagues, while still maintaining one's sense of privacy and personal boundaries, is not a unique situation for people with mental illness. Maria's following description of being circumspect in order to avoid unwanted exposure, however, is particularly significant due to the media attention that has accompanied some of the informants' prior illness-related offending.

I was also very conscious in the organisation, which I'm sure that must happen to other people coming out of my situation, is that mostly you have to learn to be pretty circumspect. So you always 
go to work with a fear going on there, and that the alienation is kind of there somehow. (Maria, p. 8)

As Maria continues on to describe, the managing of unwanted questions from work associates requires considered responses; the effectiveness of which are often enhanced by prior coaching and rehearsal: "I think it is important is listen to other people, so I was often able, as soon as someone directed a question at me, I learnt to redirect something back to them (laugh)" (Maria, p.6).

The two informants, who recounted during their interviews that their prior illness-related offending had been subject to public scrutiny within the media, also placed the greatest emphasis on their fear of being exposed at work. The extent to which they considered and managed the risk of unwanted workplace exposure is even more pronounced in light of the fact that they both described enjoying connecting with others. In the following account, Joe paints a picture of how he believes being in work heightens his risk of public exposure in relation to his illness-related offence.

Like on some of the sites I'm walking around and I'm thinking, "oh yeah, wonder if any of these guys have blabbered on to all these people on the sites". You're walking around in a dark shadow, hoping they don't tell anyone and stuff. That's the main point for me, I don't really want to go and get a proper job with different people because I couldn't sit in the same room as them, because you know, they find out your history and stuff, and think that you can't be trusted, that's how I feel, you can't be trusted, aye. (Joe, p.12)

Joe is describing some of the issues which eventually led him to remove himself from the work that he enjoyed within the open employment environment. While he continues to be very successful in his role within a consumer-driven affirmative business, his self-imposed work restrictions have also precluded him from significant economic and skills building opportunities. Joe's fear of being exposed to increased public scrutiny because of his workmates indiscretions has led to his antipathy for open employment.

Joe's situation of self-imposed alienation introduces another Freirean (1972) term; 'the culture of silence'. Among the notions that Freire aligns with the culture of silence, is that people who are marginalised within society, will then in 
turn internalise the myth of their own natural inferiority. According to Freire, they come to see themselves as the social elite see them, and needs to see them; as unreliable, dangerous, unmotivated and so on. In the above narrative, Joe recounts that he can see the potential for colleagues to regard him as untrustworthy. While he does not necessarily incorporate this view into his own self image, he sees it as a societal view that drives him to keep his distance from unknown people and workplaces. Therefore, it is the fear of having their history of illness and illness-related offending exposed, which drives most of the informants need to adopt a self-protective stance and maintain a distance from their colleagues.

\section{Being Alienated from Others}

Freire (1972) is led by a concern for people who experience cultural alienation as the result of marginalisation imposed both wittingly, and unwittingly, by the forces of hegemony that influence each society. The felt experience of being alienated from others in the work place was described by seven of the informants. As Joe exemplifies in the next extract, for some people, this was a feeling that arose from an internalised sense of being set apart based on prior life experience, rather than in relation to actual events that occurred within the workplace.

But if I went and got another job with another firm, I'd feel a bit scared 'cause I don't know them, and when you're mentally ill you're a bit scared of letting them know what you've done and stuff like that. I don't really like to actually mingle with people I don't know, who doesn't know me or my family with mental illness and stuff, but I do still love the job. (Joe, p.3)

For some of the informants, the experience of feeling alienated in the work place was in direct response to actual workplace events. In the following excerpt, Lavina describes her felt response to the shift in manager/ employee relationships that occurred as a consequence of the business changing ownership.

Well, alienated and pressured. Well, they would talk in their own language sometimes and the lady would be like "roo roo roo" and I'm like oh god (laugh). I don't know whether it was paranoia or whether it was about me..//.. (Lavina, p.6) 
While Lamina's feelings of alienation and pressure led to her questioning her own mental state, these feelings arose from workplace situations and confrontations that she reports her colleagues were also experiencing. In contrast, Rod's experience of being alienated was uniquely concerned with his mental illness and arose some months after he'd successfully resumed employment. He suspects that this alienation was driven by workplace gossip, a situation that arose after a colleague who knew his family recognised his name.

\begin{abstract}
Well, they come right up to you and look to see the craziness in your eyes and sitting way back there they are staring at you. I don't know what they were hoping to find. And then the feeling of...., it's not a good feeling to sit there and have to take it. I just carried on and had to fight the emotions coming out from that, like... crazy, crazy stigma, the looks in the eyes, yeah. So I just backed off from people for a while and just did my job. The bosses were good, I don't know whether they have seen my history, just the workers were the problem. (Rod, p. 9)
\end{abstract}

Rod is portraying the felt experience of being alienated within the workplace because of stigma. His workmates response was even more pronounced because previously Rod had felt accepted and valued as a colleague. By avoiding people and just focusing on the job at hand, Rod was responding to his emotional turmoil by remaining set apart and silent. Disenfranchised people, according to Freire's (1972) 'culture of silence' construct, are silenced because not only do they not have a voice, but they are unaware they do not have a voice. According to the culture of silence construct, people who are traditionally marginalised within a society, which includes people with mental illness, have embraced the cultural myths of the people in power. This self-stigmatisation concept is also described by author and consumer advocate, Dr Patricia Deegan (1992), who writes from the perspective of someone with a lived experience of mental illness. She details the process of internalised self-stigmatisation, and then explains the negative impact it has on the population of people with mental illness; referring to this as 'mentalism'. Within the informants' accounts, therefore, the stigmatising practises of others and self-stigmatisation mutually reinforce each other, and therefore both lead to the informants' experience of being alienated within the workplace. 


\section{Keeping Colleagues at Arms Length}

Choosing to be set apart from colleagues was also regarded as a self-protective stance by Maria, Joe, Clint and Rod. For Maria this was related to being circumspect about her self-disclosure in order to maintain her privacy with her new work colleagues. For the other three informants, however, keeping away from colleagues was a deliberate measure they employed to avert harm befalling them, either from being the victim of malicious gossip, or trouble arising from ready access to drugs and alcohol. In the following excerpt, Joe describes his rationale for maintaining his distance from his colleagues and others who have had little or no experience of mental illness.

For people who don't have illnesses or don't have people they know who have mental illness, they see us dangerous...//...That's why I'd rather them sit way over there, and I'll sit here..//.. Keeps me safer, I feel safer like, yeah. That's the way I feel. You stay there, you keep your shit to yourself and I'll keep mine to mine. (Joe, p.10)

From a critical perspective, Joe is illustrating how the forces of hegemony serve to keep disenfranchised populations isolated. Joe's experience, which is echoed both within the mental health literature and by his fellow informants, is that popular myths about people with mental illness have stigmatised this group with labels such as dangerous and unpredictable (Dorio, 2004; Lapsley, 2003). By choosing to remain set apart, Joe perceives he and his compatriots may potentially avoid being brought into question when, and if, trouble arises.

\section{Being Scrutinised and Judged on the Basis of Diagnosis}

Six of the informants described anticipating that their re-entry to work would become the unwanted catalyst for both scrutiny and judgement based solely on their mental illness. For Joe, getting a job through family associates was important, in that his colleagues knew him, and also knew his situation. "It made it a hell of a lot easier I think, I would have probably been paranoid as, if they didn't know me. They'd be judgemental and stuff and I'd be on guard all the time" (Joe, p.8). In elaborating on his firmly and consistently held position in only working with people known to him and his whanau, Joe talks further about being subsumed by a culture of fear and lack of self-belief that people with 
mental illness often experience. Again these elements are reflective of the Freirean (1972) notion about the culture of silence.

For mental illness people its basically getting the confidence I'd say, that's the major I reckon is confidence and getting used to people you don't know. That will be the biggest one for people like me, 'cause you're always being judged, yeah, simple as. I hear some of the patients have got really good qualifications but they can't go and do it because they haven't the confidence and are too scared. (Joe, p. 19)

While Terri also initially concurred with Joe's view, that disclosing her illness would subject her to judgement, when she tested this out at work she felt bolstered by her colleagues' response to her illness. By others sharing their own experiences of friends and acquaintances with mental illness, Terri's experience was one of shared dialogue creating a sense of acceptance, rather than the judgement she had expected.

Like when I opened up enough to tell them that I had been in a hospital, I sort of felt then that it made me feel they were judging me, but most of them told me that they knew somebody else who had something like bipolar or something else, you know, yeah. (Terri, p.5)

Terri's disclosure enabled her to test some of her apprehensions about being judged because of her mental illness. Consequently, she was able to allay some of her fears and ameliorate this myth. Freire (1972) states that with demystification comes a new view of reality. This new perspective also makes possible a conscientised people who approach each other in a common quest to be more human; sentiments that Louis illustrates in his following extract.

Yeah, well I suppose you don't judge a book by its cover; like the cover will be the Forensic service, the book is the person. Well, I know that and they might say, "I wouldn't want to employ that person because what do they know, their brain doesn't work because they have been 'over there'," Like no, no, no, little do they know there are some very, very clever people in "those places". (Louis, p.18)

Within this theme, Joe's narrative illustrates how he, and others affected by mental illness, are still struggling to gain the personal confidence in order to withstand judgement from others in society. Terri and Louis' comments, 
however, demonstrate a more hopeful approach to the prospect of being judged on the basis of a diagnosis of mental illness. In critically self-reflecting on his previous situation of unemployment and incarceration, Louis demonstrates how he has been able to cast aside the culture of silence (Freire, 1972). Hence, his critical awareness is melding with the reflection and action of praxis, thereby enabling him to transform his life by overcoming the stigma of mental illness with a new view of reality.

\section{Being Teased and Bullied}

Five of the informants spoke about the profoundly negative experiences of being the direct recipient of on-the-job teasing, which for two people included verbal abuse. "Probably just the abuse on the job really, that was the hard part" (Joe, p.14). Being a frequent recipient of the foreman's abuse, combined with feeling sedated in the mornings, eventually led to Joe's resignation. Joe recounts, however, that this bullying behaviour was not directly related to him as an individual, or his mental illness.

But basically I was the only one who could actually stand working with him 'cause none of the other guys liked working with the foreman. That was the downfall, everyday dreading going back to work with him there, yeah, the work was good, really enjoyed the work. (Joe, p. 2)

Like Joe, Lavina also resigned due to the bullying she received from her new employers after the business changed hands. As she described this situation during her interview for this study, she further clarified her position by contrasting the new owners' bullying behaviour, with the previous owner's short fuse under rush hour pressure:

Yeah, yeah the first owners it was just the usual pressure, the usual way a [food service] is run but these people they were like nothing you could do was good enough. Yes, and at first they were hungry to learn the trade because they'd never been in that trade before and then once they started knowing things they started pushing their weight and I started feeling really alienated and under pressure. (Lavina, p.6)

With hindsight, Lavina saw that her first employer's intermittent short fuse was due to the nature of the job. Therefore, although not desirable, she was able to 
critically reflect and see his pressure-induced sharpness as an acceptable component of the hurly-burly of the workplace. This contrasted with her experience of the new owners' unreasonable behaviour which eventually led to her resignation. Joe and Lavina are the only two informants who have not sought alternative open employment opportunities. They both see that being a recipient of workplace bullying was a significant contributor to their decision not to work in open employment. Instead they have chosen to be employed within a consumer-driven affirmative business.

Working at [the affirmative business] now, its easier to work there because all the guys know each other and they know what situation everyone's in, we can relate to each other, you know, joke to each other and stuff, but when you're in the real world, working with people who don't understand, it can be quite scary for us, well it is for me. (Joe, p.7)

In a situation of alienation, such as Joe and Lavina have described above, Freire (1972) acknowledges that people may be impaired in their use of power. During their interviews, both Joe and Lavina were able use hindsight to gain a new view of the bullying they'd been subjected to. They both concluded that they had tolerated this bullying much longer than their colleagues because of their lack of workplace confidence and sense of belonging.

Freire's problematisation process seeks to place people who are confronted by societal alienation, in a consciously critical confrontation with their problems. To this end, problematisation has a liberatory methodology, which is both problem posing and conscientising. Kiri illuminates these processes below as she describes how her critical reflection on hurtful workplace teasing enabled her to move past feeling judged by others, to more proactive responses that were both liberating and self-affirming.

But you get some people that think, "god, they're mad", you know, and I don't like that term "nutter" or being called "mad" or a "fool" or something like. I don't really care what they say. I'm a good person, I work really hard, and I treat people with respect. I'm just that sort of person that can get along with others and be genuine. So at the end of the day, after all the picking and the talking, I sort of think of myself, I'm a better person than that. I don't need to behave like them because I'm better than that. 
Yeah, that's how I get through it 'cause you do get people who judge me and know I have a mental illness. (Kiri, p.11)

As Kiri's narrative commences, she reports being labelled by some work colleagues in a manner that typifies a dimension of Freire's (1972) culture of silence. This aspect consists of the societal myths that have traditionally been imposed upon the mental health community. Joe and Lavina's response to workplace bullying was to resign and seek re-employment within a supportive affirmative business. In contrast, Kiri's critical thinking has led to her problem posing approach. Engaging in this process has enabled her to move from a culturally marginalised position, to embracing a view that enables her to see the reality as a totality, with all its causes and consequences (Freire, 1972).

\section{Keeping Safe: Staying Right Away from Drugs and Alcohol}

Louis, Clint and Joe, all of whom had been regular cannabis users prior to their forensic hospitalisation, described how their return-to-work had once again brought this drug back into their realm. While they all stated that they had continued to remain abstinent, it was a decision that had provoked some thoughtful consideration and self-reflection when confronted with the issue in the workplace. In addition to his commitment to maintaining his mental well-being, Louis describes that his desire to be seen as a role model for his whanau also drives his continued abstinence. "I'd like role model that, 'cause obviously work and drugs don't mix. You wouldn't be..., well you might be able to keep it up for a day, or I don't know, for however long, but not for ever you know" (Louis, p.14). For Clint and Joe, the reality of marijuana and alcohol in the workplace caused them considerable discomfort as they took stock of the personal implications of substances in the workplace.

So they knew my history and it was easier to relate to them because they already knew it and they knew not to give me dope on my job 'cause they know what it did to me. But they'd still smoke it on the job but not around me. When they came back to the job I could see they were stoned and it'd feel like I'm missing out and seeing them stoned. I'd think, "oh, what if I was stoned," I'd be like, you know, "what it would feel like for myself, being stoned" but yeah it was all right. (Joe, p.8) 
I was working with people who don't share the same values and so forth. People that smoke dope basically and sometimes they can work on weekends and I can work Fridays, and it can be pretty hard when you're the only one not drinking so..., but otherwise work was pretty great. (Clint, p.5)

Working with colleagues who are regularly under the influence of cannabis, both on and off the job, was stressful for Clint and Joe: "I don't know it just gave me shivers down my body, spine and stuff, gives me a little bit on edge, paranoid" (Joe, p.9). As Clint illustrates in his next extract, keeping himself safe meant retreating from colleagues who drink alcohol and use drugs, and removing himself from situations where this was likely to happen. Oh, you know, not drinking, not smoking dope, sometimes trying to avoid them, people talking about sex. Yeah, but I try and put myself in a group of people who don't talk about those things" (Clint, p.5). Keeping themselves away for alcohol and cannabis, however, added to their sense of being set apart from others within their work domain.

\section{Summary}

Within the realm of mental health care in New Zealand, since the early nineties the previously undisputed norms of locked inpatient care have been replaced with community based treatment and rehabilitation interventions. This shift to community integration, however, has been accompanied by prejudicial attitudes and negative stereotyping, which have been fuelled by sensationalist reporting in the media (Read, 2003; Smellie, 2006). The informants' experiences of both stigmatisation by others and self-stigmatisation are conveyed in their descriptions of 'being set apart'. For both Lavina and Joe, their experience was at the extreme end, and included being subjected to intimidating practises that eventually led to both of them resigning and choosing to work within a supportive affirmative business. Many of the informants' narratives, however, demonstrate that with work as a primary instigator, they have been able to emerge from their situations of social and workplace isolation. The task of the disenfranchised, according to Freire's (1972) humanisation construct, is to liberate themselves and, in the process, liberate society from their prejudicial attitudes. It is evident in most of the informants' narratives that the emancipatory process of humanisation has 
helped to ameliorate their perception of social exclusion in the workplace. For all the informants, however, their fear of potential exclusion because of having a history of mental illness and prior illness-related offending means they have adopted a hesitant and circumspect approach to their workplace relationships.

\section{Overarching Theme: Redeeming Oneself}

Redeeming oneself is the overarching theme that refers to the informants' profound experience of regaining family, whanau and collegial pride, and reclaiming a belief in oneself. As such, this was a transformative act, and for some a spiritual experience, which all the informants state was enhanced by their return-to-work. In assuming a worker role, the informants were required to reconcile aspects of their identity which will be explored within the following five themes: 're-establishing family pride'; 'others believing in you'; 'believing in yourself'; 'proving yourself' and 'spirituality resonating at work'. In addition, the Freirean liberatory quests of praxis, humanisation and transformative action provide a platform upon which to critique the redemptive features of the informants' return-to-work experience.

\section{Re-establishing Family Pride}

Louis, Kiri and Joe, all described the re-establishing of family pride as being a focal outcome of their return-to-work. In drawing a correlation between themes, it adds depth to their experiences to note that these informants were also the people who described turning their lives around after having the impact of their mental illness compounded by substance abuse (see p.88). As the informants talked about their families of origin, it became clear that many of them grew up with a visceral understanding of social, economic and cultural tensions. The informants' experiences of social exclusion because of their mental illness and illness-related offending added to these tensions. As demonstrated within Joe's excerpt, for some of the informants, this alienation also extended to their family culture. 
family situation and basically I went straight back into that family situation so that was a bit hard too. Like you left bad, and you come back and you're still bad. But Dad still stuck by me though, he still gave me the chance. (Joe, p.17)

Joe continues by describing how his return-to-work was a major contributor to regaining his family's pride.

I think that's why my brother and my father got a bit of pride in me at the end, knowing that I can go and do that (work). My brother's quite proud of me these days. (Joe, p. 19)

While Joe described a uniformly positive family response, Louis' return-to-work was met with opposing family responses. In the first instance, the regaining of family pride was a profound and multi-dimensional experience. In the following excerpt, Louis describes how his newly acquired work skills are highly valued by his family because of their transferable applications, which have wider whanau benefits.

They are all happy about it; my Dad's happy, my in-laws, my cousins and aunties they are really happy because when I go to meetings, like on our land meetings, I always contribute. So now they always want me go because I'm the only one that can contribute, well not the only one, but I got no problems getting up and saying something. (Louis, p.13)

Louis' return-to-work, however, was also accompanied by both his partner's and his unemployed brother's pressure for Louis to retain his benefit entitlements and unstructured lifestyle. The lifestyle loss that occurs when an individual moves from being unemployed into a worker role has been identified as an important consideration within the international and New Zealand mental health employment literature (Becker et al., 1998; The New Zealand National Employment Forum, 1999). In the next extract, Louis reflects on how his returnto-work continues to be a source of conflict within his relationship with his brother.

My brother, I suppose he's still not happy. There is no reason why he can't get out there and do something, you know, he just sits on his chair and watches TV all day well he needs to get out there and make a start. (Louis, p.13) 
Louis describes that while he initially felt somewhat pressured by his partner's and brother's desire for him to remain unemployed, he is now able to translate his own experience of transformative action into being hopeful about his brother's situation.

After the events that led to Louis, Joe and Kiri spending years in a secure care facility, they all viewed that returning to work had been a key factor in being able to re-establish a valued role within their families of origin. Louis, however, described a mixed response from his family. Illuminating discussions from within the literature reviewed for this study, Louis' resultant lifestyle change was not well received by two relatives who were also beneficiaries. Re-establishing family pride, therefore, resulted from the informants' transformative actions (Freire, 1972). This action has enabled the informants to move beyond the barriers imposed by some family contexts, and historical mental health practises, which regard unemployment and an unstructured lifestyle as the norm.

\section{Others Believing in You vis-à-vis Believing in Yourself}

Although arising from the informants' workplace competencies, the selfconfidence and self-efficacy that arose from having others believe in you extended beyond the informants' work domain, into enhancing both their own positive self-concept and their relationships with others. "Yeah, people really praise me for that; they let me know that's a good job and attitude” (Kiri, p.12). Kiri's experience of feeling bolstered by others can be viewed within the context of Freire's (1972) notion of humanisation. According to Freire, the central task in any movement towards liberation is the creation of a humanly-enhancing culture. The individual and collective value of a community culture that enhances each member's personal growth is captured by Maria: "People have taken time with me and given me encouragement, and I think that's the thing; people believe in you so you can keep on believing in yourself"' (Maria, p.20).

Although endeavouring to redeem themselves was part and parcel of all the informants' personal recovery journey, when practitioners, family and whanau bolstered the informants during their initial return-to-work, they engendered hopefulness into the situation. This hopefulness enabled the informants to 
perceive that their socially isolated position was not the outcome of a set fate, but merely as limiting and therefore challenging (Freire, 1972). Hence, the informants move towards returning to work, both supports and is supported by, the positive regard they are held in by their family, whanau and colleagues. "Well, I suppose, you know, it's gained me respect off everybody, and if everybody is wanting you to do work here, here and now, you know I must be doing something right" (Louis, p.4).

Within Maria's following description of the experience of loss and recovery, she portrays the arduous journey of moving from a position of personal devastation to once again believing in herself. It is this regained belief in herself that makes work an option that is both available and tenable.

You are just completely shattered, and as you start to deal with what's happened, what you've done, what it means, the grief, the sorrow, the remorse, all of those things, the angst, the everything; you have to somehow start to heal and somehow start to dream again, 'cause you got to make a life for yourself. And people help you make that life, and I think that's what happened for me. So I'm very, very lucky that way. But you have to also believe in yourself, and that can take a long time, because those things get completely crushed. So to work, you have to believe in yourself. You have to believe that, yes you can do this. Do you want to do this? Can you make a go of it? Are you going to be any good at it? All those things which people think about anyway are probably amplified because you're carrying this big history with you, but, you also learn to live where you are. That's the healing part. You don't forget exactly but you know that you have put it on the back burner because otherwise you can't function and I guess that is the healing really, yeah. (Maria, p. 14)

Freire (1972) states that the catalyst for embarking on the movement towards transformative action, such as Maria describes above, lies in the concept of humanisation. In this regard, Maria's story of redemption demonstrates that when people are able to emerge and reflect on their situation, they discover in the obstacles to their humanisation, and are thereby called to struggle against them. This call to overcoming the internalised and societal barriers that often accompany a diagnosis of a serious mental illness is encapsulated in Kiri's comment. "Well, you learn to deal with it. You have to. You sort of learn, well 
I've got a mental illness but that doesn't make me any less of a person, yeah" (Kiri, p.4).

\section{Proving Oneself}

Expressed as both a driving intrinsic need, and a valued by-product of the actual return-to-work experience, all the informants told a story about proving themselves by achieving at work, which is exemplified in Louis's account.

I just like to do the best job that I can, you know, in whatever job I'm doing. You can have all the certificates, diplomas, degrees in the world but if you can't turn up to work you're no good to anybody. So the goal is to be realistic, to be reliable and when you do get to work, do a good job..//.. (Louis, p.6)

Louis' awareness of the potential for scepticism based on the prejudicial attitudes of some members of the community is reflected in his following statement. Here Louis defends his proven work competencies from the potential accusations of 'tokenism' which he fears may continue to shadow and downplay his genuine workplace achievements.

I suppose people say now that, "jeez they (the employers) are good to you" and I say, "well you know I think I might have earned the right for them to be like that," I don't want to be just given a job just for tokenism. (Louis, p.5)

For Louis, proving himself is a two pronged process. Firstly, he relates this process to his tangible workplace accomplishments. Secondly, he identifies the need to flag his accomplishments to others, particularly to those who may intentionally, or unintentionally, try and detract from his achievements.

Rod has the most extensive work history of all the informants. For him, working hard and having a good attendance record proves your value as a worker and thereby leads to job security and other employer-driven rewards.

The only advantage I see is just doing a good job and from there on things will fall into place, and I've got that to back me up. Yeah. If you start taking days off, well then you've got nothing to back you up. When something comes along, you've got your work history to fall back on and help. (Rod, p.12) 
Freire's (1972) notion of humanisation in this return-to-work situation, translates into the informants being able to see beyond the constraints created by their history of illness-related offending, and see their future in terms of what it can be. They are then called to do something about their situation, and thereby prove their potential. Freire purports that this, and only this, is the kind of freedom human beings enjoy. It is not the freedom to realise absolute or abstract outcomes, but a situated freedom. By embracing this situated freedom, the informants have been able to address themselves in their situation of unemployment and social isolation, harness their growth potential and out of the worse create the better.

\section{Spirituality Resonating at Work}

Maria, Clint and Louis spoke about their spiritual beliefs aligning with, and being enhanced by, their return to work. In the following excerpt, Maria makes meaning of her spirituality as a personal journey, and then extends her understanding of spirituality into a wider community and employment context.

I'm developing my relationship with God and I'm developing my relationship with finding out about myself../l.. I think as I say you have to find your own resources, the whole questions of "who am I, what are my values, you know?" Even get a second chance in life, because when you come out of somewhere like the [forensic hospital] you do need a second chance, and you have to find people that will give you a second chance dealing with what's happened. (Maria, p.13)

Maria's experience of spirituality extending to community responsiveness reflects the Freirean (1972) notion that the action/ reflection cycle of praxis needs to occur in fellowship with others. Maria's description of being given a second chance, therefore, illustrates joint praxis. Accordingly, the informants' reintegration back into the world of work, and the wider community, essentially hinged on people being united by their action, and their reflection on that action, and upon the world (Freire, 1972). 


\section{Summary}

Redeeming oneself refers to the informants' felt need to reconcile aspects of their identity that are rooted both within and outside of the forensic psychiatry institution. In exploring this from a critical hermeneutic perspective, Heilman (2003) states that for those whose cultural positionings conflict with the norms of the wider community, reconciling these aspects of one's identity is an unavoidable experience. Accordingly, the informants' experience of redemption was sharply brought into question with the shift from their incarcerated world to living and working in the community. They described their need to reconcile their complex and conflicting ways of being within their families, as well as within the wider community. In this regard, Freire's (1972) notion of humanisation in this return-to-work experience, translates into the informants being able to see their situation, not only in terms of the constraints created by their history of illness and illness-related offending, but also in terms of what it could be. Therefore, it was a redeeming opportunity that allowed the informants to prove themselves under the scrutiny of colleagues, family, whanau and the wider community, rather than just the isolated world of psychiatric care.

\section{Chapter Summary}

This chapter has focused on the informants' liberatory quest wherein their returnto-work journey saw them re-engaging with colleagues and the wider community after their years of social isolation within a secure care facility. In explicating the socio-political influences that accompanied the informants' challenges and accomplishments, Freire's critical theories have informed the exploration of the four overarching themes: 're-entering the world'; 'fortitude'; 'redeeming oneself' and 'being set apart'. The next chapter seeks to add a complementary focus by looking at the pre-employment, economic and workplace aspects of the informants' return-to-work experience. 


\title{
CHAPTER SIX: AUTHENTIC REFLECTION: BEING GROUNDED IN THE HERE AND NOW, WITHIN THE REALITY OF THE SITUATION
}

\begin{abstract}
Chapter Overview
This is the second of three findings chapters that seeks to explicate the understandings that have emerged from the data. The preceding chapter was concerned with the informants' experience of prejudicial attitudes and social exclusion, and how these socio political impediments influenced their return-towork experience. This chapter seeks to add a complementary focus by exploring the more concretised experiences that are encapsulated within the overarching themes of: 'doing the groundwork'; 'weighing up the economics' and 'fitting in with workaday life'. Therefore, this section of the thesis is concerned with the informants' reflections on the actual pre-employment, economic and workplace situations in which they found themselves submerged. According to Freire (1972), people continuously transform their environment through human activity. Consequently, this chapter also explores the new challenges and tasks that continued to evolve from within these work and economic situations. The overarching themes, and the themes that constitute the informants experiences within each of the three overarching themes, are summarised below.
\end{abstract}

Chapter Six: Themes

Doing the Ground Work

Building work skills \& resources.

Training and education.

Others making re-entry to work more of a level playing field.

Affirmative EEO strategies.

Weighing- up the Economics

Tallying up costs versus benefits.

Welcoming remuneration.

Ending economic dependency.

Fitting in with Workaday Life

Becoming versed in workplace norms \& conditions.

Casualisation of work conditions.

Being acknowledged: Rewards \& promotion.

Mastering work skills. 
Fitting in with the social scene.

Getting on-the-job support.

Applying a Freirean lens to the informants' experience of their work and economic situations sees a replication of many of the terms used within the preceding chapter, including praxis; transformative action; dialogical processes; humanisation; problematisation and critical consciousness. This terminology has previously been outlined in-depth within the methodology chapter (see p. 43). The following paragraph draws these terms together in order to provide a reorientation to Freire's (1972) constructs, as well as demonstrate the interrelationships between these constructs.

Men and women are called to transformative action in order to act upon and change the human world, and in so doing so, transform themselves. Transformative action involves critical reflection on the reality of one's own situation, in order to meld the reflection/ action cycle of praxis. Conscientisation is the result of dialogical processes and transformative action that acts upon the world, which in turn leads to humanisation. This is the term that Freire uses to encapsulate the quest to be more fully human by creating a humanly enhancing culture as a fundamental means of striving for liberation. To this end, critical consciousness leads to the rejection of passive compliance in order to embrace collaborative and dialogical processes. This level of mature critique occurs through using problematisation strategies to analyse problems at a critically conscious level to avoid the distortions caused by preconceived notions and oppressive beliefs. Consequently, the critical consciousness that leads to liberation is realised not only through an individual or intellectual effort, but through collective struggle and praxis.

In addition, this chapter introduces a new construct upon which Freirean critical theory is founded; liberatory education. From a Freirean (1972) perspective, traditional schooling is considered to contribute to the marginalisation of those who have been historically disenfranchised within society. Adult education, Freire believes, tends to replicate the hierarchal mode of earlier schooling. This contrasts with the Freirean experience of liberatory education. Within this 
pedagogical process the learner-centred syllabus is focused on pressing social needs in order to challenge socio-political contradictions.

The terms outlined above are the Freirean (1972) constructs which serve to place the informants' experiences within a critical dimension. This chapter explores the informants addressing themselves within their pre-employment, economic and workplace situations. In transforming their work and economic environments, the informants are shaping the conditions for their work, and for their very existence.

Overarching Theme: Doing the Ground Work

The decision to return-to-work from their position of long-term unemployment required all the informants to invest in some measure of preparatory ground work. Accordingly, the initial themes explore the informants' experiences of 'building skills and resources' and 'training and education opportunities'. The subsequent themes, 'others making re-entry to work more of a level playing field' and 'affirmative EEO ${ }^{13}$ strategies' then examine the proactive interventions of other people, which helped the informants overcome significant workplace barriers. These are largely concerned with stigma and discrimination. In applying a critical lens, the informants' ability to mobilise themselves to the transformative actions of learning and skill building are explored in terms of the Freirean notions of humanisation, problem posing and liberatory education.

\section{Building Work Skills and Resources}

All the informants described how re-building their work skills and/or resources enabled their eventual return-to-work. "You develop your work skills again, and you develop that over time because being in an institution for several years is a long time and you lose a lot of your daily living skills" (Maria, p.19). During their period of hospitalisation, all informants had been involved in one or more 'Affirmative Business' ${ }^{14}$. In some cases, this involvement in a work project had

\footnotetext{
${ }^{13} \mathrm{EEO}$ is the abbreviated form of 'Equal Employment Opportunity'.

${ }^{14}$ The Affirmative Business approach develops partnerships between consumers of mental health services, service providers, and business people. By facilitating the production of goods and services which can be sold in the broader community, it creates job opportunities for people with psychiatrics disabilities. (Krupa et al., 2000, p. 13)
} 
extended over a period of years. In all cases, this participation was regarded by the informants as a positive precursor to open employment. For Maria, the time she spent working in different work projects, provided her with a sense of respite from the world.

When I was at the [Forensic service] and going along to the [affirmative work projects]..//.. things like that I think were really helpful because in the healing of it all, and the moving on from what's happened; the first few years are just fraught and extremely difficult. (Maria, p. 10)

Being able to hunker down within a supportive, low pressure work environment, particularly when she was at her most vulnerable, was seen by Maria as contributing towards her healing. In addition, the contribution that these businesses made to the structuring of one's day is highlighted within Lavina's following excerpt.

Keeping myself busy, keeping my hands busy that was something...//... But all the outwork, even if it was just packing, I was still earning a wage. I was keeping busy; there was the tea breaks and the lunch breaks, all that structure. Having that structure in a day helped. (Lavina, p. 11)

Lavina appreciated both earning additional money and the opportunity to learn valuable transferable skills. The two benefits she stresses, however, are concerned with having structure in her day and being kept busy. Both of these elements helped ameliorate the 'slow time' that occurs as a consequence of living within a secure care environment. In expanding further on the notion of these work projects helping to remediate the tensions inherent in being locked in and set apart, Louis identifies another intrinsic benefit; socialisation with colleagues:

Or sometimes you might not like the work but you might like the people you work with. So you can have a joke and stuff and get you through the work. Maybe that's how you get through the work, you know, just joking around with your work mates. (Louis, p. 15)

Even though the manual labour involved in this work project did not align with Louis' work preferences, he was able to critically reflect on the motivational value of the camaraderie that he had with his work colleagues. 
For both Rod and Terri, work within a consumer project became a gateway for returning to open employment as soon as their legal restrictions allowed this to occur.

Working at [an Affirmative Business]. Yeah, it got me back into doing something for a pay. Yeah, it gave me an incentive to go out and get work again. (Terri, p. 2)

The packaging was so boring but I just stuck at it, and I don't know if it was just natural, but if I saw a job that needed to be done, then I would just do it and then I felt easier about going to work. (Rod, p. 5)

While Rod and Terri felt that this groundwork gave them an incentive to go out and seek work, Lavina's next extract highlights a cautionary perspective.

\section{I think the physical and the mental challenge at the Affirmative Business were easy going, things at cleaning were easy going, but this was a pressure thing and I found that hard, like I must admit the first few weeks, the lunchtimes I would dread (laugh). (Lavina, p. 9)}

In contrasting the difference in pressure between her food services job with the low pressure affirmative business environment, Lavina is illuminating some of the findings in the vocational rehabilitation literature reviewed for this study. The literature concurs that significant differences between sheltered and open employment, in both requirements and opportunities, suggests caution when making generalisations between the two setting types (Anthony et al., 2002; Mueller \& Wilgosh, 1991; Schneider, 1998b).

Prior to re-entering the open work environment, all the informants had worked in an affirmative business. These businesses ameliorated the 'slow time' associated with their secure care hospitalisation by offering structure, camaraderie and activity. They also helped some of the informants to build skills and resources that eventually assisted their return-to-work. These low pressure work environments, however, did not fully duplicate the demands of the current employment environment. Some of the informants found that in terms of the speed of their work output and their work stamina, they were not fully prepared for their return-to-work. From a Freirean perspective, the informants were only able to mobilise themselves to transformative action in terms of what Freire 
(1972) describes as their human situation. This is the situation that holds creative possibilities, wherein the informants were able to address themselves within their situation of unemployment and secure care hospitalisation. By doing so, the informants were able to start building the skills and resources that would eventually shape their work future.

\section{Training and Education}

Six of the informants described how an array of formal and informal training and education opportunities had ultimately bolstered their success at work. Maria had previously succeeded well both at school and in her tertiary studies. "I suppose for me too, I was lucky I'd had a fairly good education” (Maria, p. 18). Prior to her return to work, Maria undertook further educational opportunities as part of her carefully planned preparation for work. This plan saw her up-skill her computer skills by accessing training courses offered in-house by two mental health agencies. "Thank goodness I did that (the computer course), because at least it gave me some sort of preparation for what I was going to need. So one of the big things about this is like you ask yourself, "well, what can I do?" (Maria, p. 4). Feeling bolstered by her initial success in computer training, Maria then progressed her return-to-work plan by enrolling in a social sciences course offered by a large tertiary institute. In reflecting back on her decision to engage in further training, Maria describes below the advantages of a stepwise approach in moving from an education setting into work.

I think coming out of a forensic situation like that or a psych hospital like that to go straight into employment could have been quite difficult but the course was a good practice area in terms of social skills, just talking to people again, as I say limbering up your brain, all that kind of stuff. (Maria, p. 2).

In contrast to Maria's positive experiences with training and education, the only other informant to attend a training course prior to returning to work was Joe, who reports a wholly negative experience.

They (the other course participants) were there just to mingle and get drugs, and I was trying to get out of that situation because, you know, I was bad on drugs in [forensic unit] and stuff. I was wanting it, but trying to keep away from it so I could get out of the 
[forensic unit], and I was actually in the same situation with the people around me. (Joe, p. 18)

In addition to the unhelpful influence of people engaging in drug taking activities, Joe believed that the trade skills he wanted to develop further were best learnt on the job.

You've actually got to go and learn it first hand actually on the job. There's no way you can sit down in a classroom and write what you're going to do 'cause what if you're a job and it's nothing to do with what you do on paperwork. (Joe, p. 17-18)

Joe reports his primary school education had been highlighted by his frequent absences, which in turn, had resulted in him not gaining reading and writing skills. 'I'm not the brainiest person but you don't need to write and write to get ahead in life I suppose, oh you do in some jobs, but honestly there will be trade jobs all the time, yeah" (Joe, p. 19). Joe's belief in on-the-job training being the most worthwhile option is shared by most of the informants.

Rod's experience of job seeking was that having no formal qualifications, along with his age, was a major impediment to securing employment despite his extensive work history. "The age factor and the qualifications factor, that's about it. The ethnic didn't seem to come into it, they had policies there. Qualifications, they always asked for qualifications" (Rod, p. 5). Since his discharge, Rod states that his job seeking endeavours have highlighted formal qualifications are now standard employment prerequisites. While Rod reports having enjoyed school, he left at fifteen to commence work and make financial contributions to his family (as described in 'revitalising work ethos' p. 81).

Seven informants described the link between their work, and their education and training experiences. Their responses to these education opportunities, however, were very mixed. Learning on-the-job was the preferred training method for most of the informants, although Rod, who had the most extensive work history, also expressed an interest in having the opportunity to prove himself within an academic programme. While Maria and Lavina's academic success at both secondary and tertiary levels enabled them to embrace further formal learning opportunities, the other informants' previous limited scholarly success made them more wary. In viewing this wariness from a Freirean (1972) liberatory 
education construct, adult education, according to Freire, generally replicates the hierarchical patterns of earlier schooling. In contrast, Freire's liberatory education focuses on participation and a learner-centred curriculum. He argues that these egalitarian measures protect the rights and opportunities of people who have been traditionally marginalised within formal education settings. In line with this Freirean thinking, most of the informants preferred on-the-job learning that employed a multitude of teaching modalities, including visual demonstration and hands-on practise, within a worker-centred curriculum.

\section{Others Making Re-entry to Work More of a Level Playing Field}

People who are hospitalised within a forensic psychiatric setting have intensive, individualised treatment and rehabilitation regimes, which are supported by high staffing levels. As Maria describes, this high level of available expertise and support contrasts sharply with the staffing resources available within the wider community based mental health sector.

That's what people perhaps don't realise, they think that society still thinks that if you go into somewhere like Forensic, you're mad and you're bad, which isn't true. But in fact you're actually more supported and more prepared than a lot of people who have to just deal with their mental illness because community services are lacking, and you're lucky if you get the kind of support that you need because there's just not the numbers of staff and maybe a lack of understanding. 'Cause I feel that I have been well supported and I would never have got back to work, I don't think, if I hadn't been, yeah. Or it would have been an even harder journey. (Maria, p. 11-12)

Along with the informants' drive to achieve their work goals, comes an initial move away from being solely reliant on District Health Board (DHB) Forensic Psychiatry. This shift to receiving services from mental health Non-Government Organisation's (NGO) with specific vocational contracts brings into question the stigma and discrimination that can often impact on people with a forensic background from within the wider mental health sector.

When I entered the [mental health NGO work service], again, even though they were working with mental health consumers, it was still quite a big deal to know what to say to them. However, on the first interview I did sort of say, "look this is my background are you prepared to work with me". Because you do 
feel for a lot of that time that the world doesn't really want to know you, and so it takes quite a lot to push yourself to go and say to someone, you know, "am I okay, really are you prepared to work with me". And anyway they were very good and, of course, very accepting and recognised that I was another consumer and I started working with [employment consultant]. (Maria, p. 2-3)

Dedicated vocational practitioners, career advisors and job brokers from both DHB and NGO services, worked intensively with six of the informants in order to enhance their employment outcomes.

To have people to practically help you with things like CVs, or going on to interviews with you, or people who make suggestions, or people who just can offer any kind of support that gets you there. I'm totally grateful for all that, because I think, yeah, how do you do it otherwise? (Maria, p. 12)

The complexities involved in this role shift, however, require astute and timely follow-up support by the forensic nurses. As Maria illuminates in the following excerpt, the forensic nurses are the professionals involved with the return-towork process, who are at the coal face of managing both risk factors and ensuring their clients' mental health.

I had been to some job interviews which were good practice because I had gone to one interview, and well the stress involved with that! I remember having to talk to [forensic community nurse] about it one day we were sitting in the house and he was very good. I was anxious and [the nurse] was always really great when I'm like that because he sort of calms me down, and helps me to think things through and stuff like that. (Maria, p. 5)

North American research has identified mental health staff attitudes as a barrier to people with mental health disabilities seeking employment (Anthony, et al., 2002; Baron \& Granger, 1997; Marrone \& Golowska, 1999; Pratt et al, 1998). As Joe exemplifies below, however, all the informants reported that the forensic staff involved in their care were supportive and encouraging of their return-towork. "The support I had from [forensic community nurse] and them, that was pretty cool. They were all for it, they were all for it, yeah". (Joe, p. 10). In support of this view, Louis also reflected on how the treatment and rehabilitation interventions that occurred during his stay in a forensic hospital were significant contributors to him securing a job following his discharge. "Yeah, well suddenly the work that was put in from people from the [forensic hospital] ... You know, 
they put a lot of work into it, not just my [social worker], but everybody, yeah" (Louis, p.15). In further clarifying the 'how and what' of practitioner helpfulness in regard to his work success and satisfaction, Louis provides a succinct, retrospective view.

I suppose the main one is that people were supportive. No use telling somebody that I want to get a job and "oh, you'll never be able to work again". You need to be, well not babied, but you know, sort of like constructive criticism is all right, as long as it does have value or there is some substance to it. Well, teaching or getting you back into a routine. Even things like showing you how to use a diary properly 'cause that's your main organisational tool I suppose. (Louis, p. 16)

Louis appreciated receiving astute feedback and encouragement, and being taught skills that he was able to successfully transfer into the work environment. In this regard, Maria also highlights the importance of acquiring skills, confidence and knowledge in modern day job seeking skills. "For starters, I didn't have confidence on the computer to be looking up jobs. I needed help with that. I didn't have the confidence to do my CV and things like that". (Maria, p. 3)

In addition to people being assisted back into work by forensic rehabilitation and vocational practitioners, three of the informants secured their jobs through their family or whanau networks. Joe's next narrative about the job market that operates out of his local pub is punctuated by the fact that he secured a position by accepting a job offer which occurred prior to him actively job seeking. "I was in the pub with Dad; one of my Dad's friends owned his own business, and he offered me a job" (Joe, p. 1). This pub based job market is centred on trade positions, mainly within the commercial and domestic building industries. In contrast to the ease of Joe's job seeking endeavours, Maria required a greater level of assistance from both practitioners and friends, in order to overcome the barriers to employment that the corporate world presented. Below, Maria reflects on the overlapping and multi-dimensional nature of the supports that eventually enabled her return to work.

My other life experiences that helped me were that I was getting support, as we talked about, from people from [DHB work service] and [NGO work service], just background support from family and friends. But also what was important was the support 
from the friend that said this job is a possibility for you. (Maria, p.18)

Maria's narrative continues on to describe how her friend's proactive intervention had secured her a job within the corporate sector, thereby breaking the cycle of rejections that had accompanied her initial foray into job seeking.

I did a good job for them, I know I did, they were very happy with me. So I had that reward to know that I was actually successful in what I was doing. But if my friend hadn't helped me get there, I might have been waiting a lot longer. So maybe people need those breaks in their life as well. (Maria, p. 12)

Six of the informants received job seeking support, and as Joe and Maria's above stories exemplify, the level of assistance required varied considerably from person to person. In addition to assistance being an individual prescription based on the informants' level of skill, the degree of support required was dictated by the specific requirements of the different employment environments. The informants received support from a number of different avenues including family and whanau; forensic psychiatric staff; vocational practitioners from both DHB and NGO services and other government agencies such as Career Services and Work and Income NZ (WINZ).

In contrast, both Terri and Kiri secured their jobs independently, without any follow-up practical help or moral support from their family or vocational/ mental health practitioners during the job seeking and job securing process. "Yeah, I ended up getting an application from the shop. I think they were advertising in the paper and then I went and got an application and filled it in" (Terri, p. 2). In addition, their autonomous approach to job seeking had been repeated on at least three occasions and each time met with success. "I just rang up and went for an interview, yeah and so got the job. No application to fill out" (Terri, p. 2).

Terri and Kiri were the only informants who secured employment without any assistance from others. They did not report, however, experiencing some of the more complex workplace barriers related to ongoing media scrutiny. In contrast, the other informants described how their re-entry to work was assisted by professionals, within both the forensic psychiatry and vocational support sectors. In addition, three of the informants were also assisted by family and friends 
whose connections within the employment sector actually secured the informants' employment. From a Freirean (1972) perspective, the very situation of being marginalised within society, as occurred for the informants because of their history of mental illness and illness-related offending, leads to a loss of selfdetermination and self-efficacy. Therefore, they become submerged in their situation and, as long as they remain so submerged, they cannot be actively engaged in the struggle for their own liberation. Consequently, the informants require access to the skills and resources possessed by vocational rehabilitation specialists, forensic psychiatric staff, and/or family and whanau, in order to seek, secure and keep employment.

\section{Affirmative EEO Strategies}

Five of the informants talked about their re-entry to work being eased by affirmative initiatives. These actions were either government strategies or driven at a local employer level. In this regard, there are a number of government led fiscal initiatives to encourage return-to-work opportunities for people who have been long-term beneficiaries, and who are thereby disadvantaged when seeking work (Ministry of Social Development, 2001). For example, Joe's employment was subsidised by WINZ during the first twelve months.

\footnotetext{
I think I was getting about $\$ 10$ per hour. But the second time when I went back I think social welfare gave him $\$ 200$ a week to keep me employed. They paid him to keep me employed for such a long time, and after that time they stopped giving it to him and then he pays my wage. (Joe, p. 3)
}

In addition to these financial strategies, individual employers also responded to the call to social inclusion by providing flexible return-to-work accommodations. As Louis illustrates in the next extract, these accommodations were tailored to each individual worker.

So I think it's sort of like a bending in process, you know, where you just starting building up your hours, and especially if you've got trouble, well for me, losing out before you even start work. Starting small and building from there. (Louis, p. 7)

Louis describes a "bending in process"; employer flexibility which is enabling of worker performance as opposed to impeding. This is a similar scenario to the 
move away from the nine to five work ethos that dominated the New Zealand workplace prior to the impact of the women's movement in the early 1970s. This is when feminism became a powerful driver towards the proactive inclusion of women in the workplace with strategies that included equal pay; part time and flexible hours, and job share arrangements. These flexible work policies have been found to promote inclusion for other groups of people who have historically been disadvantaged within the New Zealand work environment, including people with disabilities (Mintrom \& True, 2004; Minister for Disability Issues, 2001). From a Freirean (1972) perspective, the process of emerging from a position of disadvantage within the work environment requires employers and prospective employees to use a problem posing approach in order to develop mutually beneficial solutions. These solutions may include accessing dedicated government funding and/or developing individualised workplace accommodations that are able to flex around the employee needs, and thereby enhance their workplace potential.

\section{Summary}

For all of the informants, re-entering the open workforce required them to engage in preparatory groundwork. This included building skills and resources within affirmative business opportunities and/or drawing on training and education opportunities. Whether within an affirmative business or drawing on past learning and work situations, learning on-the-job was the preferred training method for most of the informants. This method of learning reflects the Freirean (1972) experience of liberatory education wherein the learner-centred syllabus is focused on the learner's personal experience and their environmental requirements. In addition to this personal learning and skills building preparation, six informants had their re-entry to work bolstered by the proactive interventions of others, including friends; family; health and vocational practitioners and prospective employers. In contrast, Terri and Kiri's autonomous job seeking endeavours that saw them both secure positions with relative ease.

From a Freirean (1972) perspective, the informants were only able to mobilise themselves to the transformative actions of education and skills building by emerging from, and critically reflecting on, the facts and circumstances of their 
unemployment. They were then called to do something about their situation and therefore did the groundwork in order to realise their work aspirations. This preparatory groundwork awakened in the informants the expectation of change in their position of long-term unemployment, thereby instigating their transformative role shift from beneficiary to worker.

\section{Overarching Theme: Weighing-up the Economics}

Prior to being employed, all the informants had been receiving either a sickness or an invalid's benefit. Their shift to a worker role had meant that all of them had either completely, or partially, replaced their benefit with wages. During their interviews, all the informants commented on the financial consequences, often unexpected, which had arisen due to this shift in their income source. Accordingly, their experiences of weighing up the economics of being in work are explored within the following themes: 'tallying up costs versus benefits'; 'welcoming remuneration' and 'ending economic dependency'. In applying the critical lens, these financial implications and consequences are explored in terms of the Freirean notions of praxis, transformative action and critical consciousness.

\section{Tallying up Costs versus Benefits}

On returning to work, it soon became evident to all the informants that they needed to tally up their work-related costs, and weigh these up against the financial rewards and other work related benefits. For all the informants, this meant managing on a tight budget. Rod and Maria, who both had considerably more work experience than the others, described being able to predict and plan their expenditure in relation to their wages. "Being able to plan to get what you need, whereas before we had to keep hold of your money, keep an eye on it" (Rod, p. 6). As Louis summarises in the following excerpt, however, the other informants all expressed considerable surprise, and some dismay, at how their work related costs, including the loss of government subsidies, had eroded their weekly income. 
And that's the thing that a lot of people on any benefits are mindful of, is that you might get a job and you might get good hours but the more you work, the more WINZ take away from you and sometimes you think that when they have done their readjustments you think that's not really worth working. Once I've done a forty hour week and paid for my transport; my food at work and to buy stuff, you're not much better off from being on the benefit. So it's a stop off for a lot of people. But for me, yeah, I think I can see the..., I suppose the light at the end of the tunnel. (Louis, p. 10)

While Louis' financial autonomy is an influencing factor, his decision to be in work is heavily weighted towards social inclusion and personal satisfaction. In the above extract, Louis also describes some of the financial barriers people who are on WINZ benefits need to consider when they return to work. In this regard, Maria describes needing to purchase new clothes in order to comply with the corporate dress code: "I had to borrow money if I remember rightly, to buy some stuff because I didn't have enough" (Maria, p. 16). While Maria was aware that she faced significant clothing costs when she secured a corporate office job, some work-related costs are unexpected. As Joe portrays in the next excerpt, unexpected expenditure is particularly burdensome for people with limited financial means. On commencing his new employment, Joe's fingers, unaccustomed to the abrasive nature of his primary work task, would be raw and sometimes bleeding at the end of the work day.

I clicked on eventually and took leather gloves and they wouldn't last you a week. You would have to go and get a new pair, and you'd get holes in the fingers in about four days. I've asked the boss for leather gloves and he just turned "nah, nah harden up". (Joe, p. 13)

In addition to being required to pay for his own protective gear, Joe faced another unexpected work-related cost when his Housing New Zealand rent had an upward adjustment in line with his increased earnings.

The money wasn't too great. The boss was paying me about 280 bucks and gave me 20 bucks gas. But I was living in Housing NZ at the time, then they found out I'm on a partial benefit and then Housing New Zealand went from $\$ 47$ a week up to a bit more. (Joe, p. 3) 
In addition to this Housing New Zealand rental adjustment, the part-time wages Joe earned at the minimum adult rate meant his WINZ benefit was proportionally reduced. Although Joe was still financially advantaged by replacing his benefit with wages, after weighing up the overall costs of work, and balancing this with the realities of his pay check, he concluded that these financial benefits were marginal.

The first time I started working there, I had big dreams what I was going to do with the money and stuff, really big dreams. But when you're working and you get your pay packet and at the end of the week you look it and you go, "oh the amount I've just done, it's not really worth it”. (Joe, p.13)

Like Joe, Clint also had substantial dreams about what his wages would enable him to achieve, which he describes in the following sequence of narratives. While Clint is happy in his current employment, and highly values the ongoing nature of his employers' flexibility and support (see p. 149), the remuneration he receives is considerably less than his desired $\$ 18$ plus per hour.

I'm looking at about $\$ 18$ an hour. Most of my friends have got work and they are getting paid like $\$ 30$ an hour. My mate who's a manager, he's getting paid $\$ 40$ an hour for managing. You know, you sort of are a good person when you hang around with people who get paid well, but...., yeah, you feel like you're the dumbest fella. (Clint, p. 10)

In relating the level of pay to status, Clint highlights how an individual's social status is often a reflection of where their worker role is positioned within the workplace hierarchy. In addition to Clint's perception of his lowered social status due to his level of remuneration, he perceives it as a barrier to establishing his highly desired goals of a long-term intimate relationship and a home.

I want a home, and still my opinions are money, money, money. With the money that I'm earning, it's not enabling me to find a partner, and to support a girlfriend or a wife in the way that I should. So it's something that I need to work towards and hopefully I can succeed, and find a job that pays well and enables me to support my girlfriend. (Clint, p. 7)

Clint is successful in his current work environment, and he highly values his flexible work hours. His overall workplace satisfaction, however, is heavily 
weighted towards receiving an hourly rate of $\$ 18$ plus. Hence, Clint perceives this will be the factor that drives him to seek alternative employment, thereby providing him with the financial means to achieve the home life he strongly desires.

A positive outcome of returning to work was that all the informants were financially better off. With the exception of Rod, however, whose full time salary was supplemented by overtime, the informants continued to live on a very tight budget. While this situation did not create an economic decline for the informants who had previously relied on a benefit, their return-to-work was typified by low hourly pay rates and part-time hours. Hence, the wages received by the informants, in most situations, resulted in only a minimal increase in available income. As Maria describes below, this situation of limited financial resources resonates throughout the wider community of people who are affected by mental illness.

I think that probably for lots of people like myself coming out of a mental health facility, or dealing with a mental health experience, is that you never have much money really. I mean I feel like now in my new job that I have a little bit more than at the first job to go on, because I am working longer hours but it's the pressure in life. (Maria, p. 16)

Both nationally and internationally, this community has identified financial resources, employment resources and vocational skills as the areas that still present the greatest barriers to community integration (Mallik, Reeves \& Dellario, 1998; Sayce, 2000). According to Freire (1972), initiating transformative action occurs within the context of a joint project. Hence, any transformative movement towards improving the financial position of people with mental illness means that this community must be helped to emerge from their impoverished position, in order to be able to intervene in it. Access to work and financial resources, therefore, remains one of the biggest challenges for people with mental illness, and for the people who support and work alongside this community. 


\section{Welcoming Remuneration}

While all the informants spoke about the need to tally up the costs of work and weigh these up against the benefits; they also all described welcoming their remuneration. As Maria reports below, the experience of earning money again, sharply contrasted with the situation of reduced benefit entitlements that accompanied the informants' secure care hospitalisation: "Actually that was quite amazing; having some money. You know, I had gone for so many years with virtually nothing at all and I didn't have capital saved up or anything like that" (Maria, p. 15). Re-experiencing a sense of financial freedom, albeit from a very limited financial pool, was a highly valued return-to-work outcome for all the informants. Although everyone acknowledged that their wages needed to be carefully budgeted, as Maria portrays below, this income also enabled the informants to experience significant life opportunities that the lack of financial resources had previously precluded. "I felt grateful, for example, now to have a little flat which I couldn't have afforded beforehand" (Maria, p. 16). Being in work has also positively impacted on her living situation, with Maria realising her goal of living alone in a small flat that is located in a desirable suburb.

From Clint's cultural perspective, his earnings have enabled him to fulfil an important cultural role. Clint spoke about the pride he felt in his new found ability to make a financial contribution to his parents and to his siblings. " $M y$ family, they call me about once a month and I give them some money. I have a chance to support them, like that's the ultimate for me, supporting my family, yeah" (Clint, p. 7). Both Clint and the cultural worker who accompanied him during his interview, described Clint's financial contribution in terms of a valued cultural role that is esteemed by the recipients within the family, and the wider Pacific Island community, as well as by the contributor himself/ herself.

The informants initial expectation of having more spare change was somewhat impeded by the combination of low hourly rates; part-time wages and newly acquired living costs. In the following comment, however, Terri encapsulates the experience of achieving a measure of financial autonomy; albeit much more humble material outcomes than many New Zealand workers may identify. " $A$ bit of financial independence; petrol in the car, food in your belly” (Terri, p. 12). 


\section{Ending Economic Dependency}

For Maria, Louis and Rod, one of the self-esteem enhancing events that occurred when they started earning wages was being able to terminate their economic dependency on the State. This driving commitment to being self-supporting is reflected in Rod's following comment: "I felt uncomfortable on WINZ, being on the dole. I knew I could do the job; I was able to carry a full day's work" (Rod, p. 6). As this theme is explored from Maria, Louis and Rod's position, it is clear that their strong work ethic and drive for financial autonomy are interwoven. After a long work career that commenced when Rod was fifteen, his only experience of being financially dependent occurred when he was a forensic inpatient: "Now I don't ever want to go back on WINZ again or welfare if I can help it..//.. Makes you feel useless" (Rod, p. 7).

Louis also experienced a very compelling desire to return-to-work. On being offered a part-time position that strongly aligned with his job preferences, Louis accepted it, with no regard to how his new income stream would impact on his or his partner's benefits, much to his partner's chagrin. "I suppose she was being a bit selfish, she thought it would affect her benefit. So I never really looked into it with WINZ about what would happen to my benefit, her benefit, and both our benefits” (Louis, p. 12).

Maria's discharge from forensic services saw her initially settling into a flatting arrangement with friends, and then commence job seeking in earnest. Hence, this shift was accompanied by a number of meetings with WINZ as Maria's social and economic positions continued to change.

But there was a woman who was a WINZ case worker and she really blew hot and cold. Sometimes she was dreadful, she was like a gatekeeper. She was shocking and questioned absolutely everything, wouldn't let you have this, wouldn't let you have that, didn't tell you about things you were eligible for. And then occasionally you'd catch her on a good day and she could be quite supportive. So you never knew what she was going to be like, but generally she was pretty difficult. But our social worker was really on to it, and she would keep trying and she wouldn't let this woman get away with all of that stuff. But what happened for me was that fortunately this woman went away for a week or two and another WINZ worker stepped into her place and she said, "of course you are eligible for that, of course you are 
eligible for this, how can we help you?" And that made all the difference. So at least I had a little bit behind me. (Maria, p. 1617)

In the above excerpt, Maria is reflecting on both her positive and negative experiences with WINZ during the period of changing entitlements which accompanied her role shifts from being a newly discharged beneficiary, to that of being a part-time worker. Maria saw this as a very mixed experience, with her access to financial assistance being dependant on her social worker's advocacy, and the individual case manager's interpretation of both the eligibility criteria and Maria's situation. Therefore, Maria perceives that an individual case worker can assume discretionary power over an individual's economic position, and that the application of this discretionary interpretation may not be fair and just. Maria's perception of the rather arbitrary nature in which WINZ financial support may be provided to those in society who are in the most need, is given even more weight in her following excerpt which commences with her initial return-to-work impressions.

It was quite an elating feeling but it was also quite scary. It was like I really hoped that everything would go okay, because I wanted this whole thing to work very strongly and passionately. But I suppose because I had never lost that kind of belief that if you can support yourself, then you should support yourself, so it wasn't that I felt like, "oh WINZ owe me" or anything like that. I didn't feel like that, I was extremely grateful to get money from WINZ. I always thought, "wow they're giving me money". (Maria, p. 17)

Maria's narrative demonstrates that it is not a sense of self-entitlement that motivates her to seek out her available WINZ entitlements, but a desire to have a responsive government-led financial support system that operates on the principles of openness, fairness and equity. From a Freirean (1972) perspective, there are negligible societal or individual benefits when a state institution, whether educational, health or financial, employs a provider-recipient approach. This is an approach that finds solutions for the people, instead of with the people, and then imposes these provider constructed solutions on the recipient. When support, including financial support, is required, Freire (1972) argues that the individual should be encouraged to be active and responsible. Ending economic dependency was a desired outcome for some of the informants, and returning to 
work achieved this goal. The resultant shift from beneficiary to worker required the informants to work collaboratively with WINZ, often with health practitioner support, in order to smooth the financial transition.

\section{Summary}

The informants' return-to-work was typified by low hourly pay rates and parttime hours. The wages they received, in most situations, only resulted in a minimal increase in their available income after work-related costs had been deducted. Regaining a sense of economic freedom, however, albeit from a very limited financial pool, was a highly valued return-to-work outcome for all the informants. Although everyone acknowledged that their wages needed to be carefully budgeted, this additional income did enable most of the informants to achieve valued goals such as improvements in housing. Both internationally and nationally, people affected by mental illness have identified financial resources, employment resources and vocational skills as the areas that still present the greatest barriers to community integration (Mallik, et al., 1998; Sayce, 2000). In accordance with Freirean (1972) theory, overcoming these barriers requires both service users and providers to initiate transformative action within the context of a joint project. Accordingly, as the informants were transforming their world of unemployment and prior forensic hospitalisation, they often required their health practitioners' support in order to be active and responsible when working in tandem with WINZ, in order to smooth their transition back into the workforce.

\section{Overarching Theme: Fitting in with Workaday Life}

The informants' experiences of the complexities and accomplishments that accompanied their endeavours to fit in with workaday life are reflected within the following seven themes. The first two themes are concerned with workplace norms and conditions: 'becoming versed in workplace norms and conditions', and 'casualisation of work conditions'. The next two themes then explore the informants' experience of workplace rewards and skills: 'being acknowledged: rewards and promotion', and 'mastering work skills'. Following this exploration of the informants' performance within workplace, this overarching theme then concludes with the last three themes looking at the informants' responses to 
socialising, workplace anxieties and employer support: 'fitting in with the social scene'; 'seeing the workplace as scary', and 'getting on the job support'. Throughout this overarching theme, the informants' experiences of fitting in to workaday life are scrutinised in terms of the Freirean notions of the culture of silence; humanisation; problematisation and praxis, which serve to view the informants' experiences from a critical perspective.

\section{Becoming Versed in Workplace Norms \& Conditions}

As consistent with any new employee's workplace experience, all the informants described their initial anxieties and the multidimensional complexities of adjusting to the prevailing norms that exist within a new work environment. These dilemmas are both encapsulated and exemplified within Louis' following excerpt:

Oh, anxiety, you know, "am I going to be able to handle it? Am I going to be able to get to work?" You know, get up and get to work, you know, the hours that I'm in, after work. Maybe staff, even though it's a supportive organisation, maybe they might have felt uncomfortable with me being there. Maybe, I don't know. There was lots of little things that I had to learn, like the confidentiality. Well not so much, I knew that that's a norm, but to know what is confidentiality and what you can actually talk about. You know, you might be talking to some other people and you're not sure what you can say and what you can't say. (Louis, p. 9)

Confidentiality is an important workplace requirement, and as Louis demonstrates by raising it as an example, the rules associated with workplace confidentiality are inherently complex to learn and apply. Therefore, it is a requirement that can only be tested and thereby fully integrated by reflection and practise within the workplace context. This process illuminates the Freirean (1972) notion of praxis; the cyclical process of action-reflection-action that eventually leads to transformative action. Successfully integrating workplace norms, therefore, is heavily reliant on the action/ reflection cycle of praxis. This cyclical action is illuminated in Louis' description about how his learning melds with his workaday life.

You know, well learn it, learn as much as I can. And it's easily learnt because you're working, you're getting paid, you're doing a job that you like anyway. But you're also learning what you 
want to learn, so I suppose you are getting paid to learn it in a roundabout way. (Louis, p. 8)

When environmental norms are clearly stated in either verbal or written directions, they are regarded as explicit requirements. Conversely implicit requirements are those norms and understandings which are also important environmental requirements, but are not directly stated. These requirements are often the behavioural subtleties that one only becomes aware of when they are unknowingly transgressed, for example, drinking out of the boss's tea cup. Understanding and adhering to both explicit and implicit requirements is important in securing one's success in any environment (Anthony et al., 2002). People with psychiatric disabilities, however, may have more difficulties with the subtle social nuances associated with implicit requirements (Anthony et al; 2002; Bedell et al,, 1998; Strong, 1998). This is demonstrated in Joe's following faux pas when he neglected to use the foreman's correct name when he was on the job.

Like one day I said to the foreman in the van when we were eating lunch and I said to him, "oh [foreman's nickname]..." and he just goes, "don't call me fucking [foreman's nickname], you fucking..." and my other workmate turns around and goes, "what? what's wrong with that?" and he goes, "he should pay a bit more respect on a job". And I was like, I call him that in the pub and I can't call him that on the job? (Joe, p. 4)

Like Joe, most of other the informants described adjusting to the social norms within their new work situation. In the following excerpt, Lavina talks about the challenges in adjusting to the social norms within an entirely different and unfamiliar social context after the familiar, low pressure environment of the affirmative business.

Like working at the [affirmative business] I'd see people there I'd know, I knew what's happening to me ..//.. and so that was the difficult thing being put in a different environment, a totally different environment with people I didn't know that was hard, but at the same time exciting, you know 'cause I got to see some famous people and my friend would be like, "ooh, who did you see today?" (laugh). (Lavina, p. 9)

Stepping outside of her familiar surroundings was difficult for Lavina, however, this shift out of her comfort zone allowed her to also experience herself in 
relation to others who have a very different socio-demographic background. When asked to reflect back during her interview, Lavina was able to perceive that it was the challenge of the unfamiliar that enabled her to grow. People who she'd only related to by seeing them in the media were now the people being warm and accepting of her, regardless of her mistakes while she was gaining her feet in her new job.

Busy executives are really nice people, really patient, because if I took a while to take an order or made a mistake they were pretty easy going ..//.. they were really good like that, yeah, and it was a real learning curve, it was a real learning curve on how to handle yourself when you're under pressure, how to focus. (Lavina, p. 10)

Working in the food industry required Lavina to learn how to manage her stress levels when under pressure. In the next excerpt, Lavina describes feeling assured in the knowledge that she was doing her best to carry out her assigned work tasks during the lunch hour pressure.

../.. the boss came at the back and he was doing the job like this, you know, and he was really exaggerating and really angry and I was trying to get in there to do the job and I felt like saying, "I'm doing my best, you know, it's busy," you know. But I took it all in my stride really, yeah, I just thought to myself, oh never mind (laugh). (Lavina, p. 10)

As Lavina had integrated into the food industry norm of rush period pressure, she was able to employ this knowledge in order to take the implied criticism about her work performance in her stride, relating her boss' comments to the lunch hour rush, rather than being daunted by them.

For most of the informants, conforming to the explicit workplace norms that are clearly and overtly stated was considerably easier than adjusting to the more subtle nuances of the implicit requirements. While this is true for any new employee, in most situations the informants' experience of becoming versed in workplace norms concurs with the literature which suggests that people with mental illness may experience more difficulties with these subtle social nuances (Anthony et al., 2002; Bedell et al., 1998; Strong, 1998). Although this theme encompasses experiences that presented the informants with some significant workplace challenges, most of the informants describe eventually being able to 
meet these requirements and grow from the experience. Therefore, the informants' endeavours illuminate the Freirean (1972) notion of humanisation which calls people to be self-creating. In taking an active role in transforming themselves within the challenges of their new work environment, the informants are also shaping the conditions for their other life domains.

\section{Casualisation of Work Conditions}

Since the introduction of the controversial and now superseded Employment Contracts Act (1990), the issue of increased casualisation of employment conditions within the workforce has been often debated within the New Zealand literature. Casualisation of workplace conditions has been the outcome of an international socio-political shift within the employment environment (Ansley, 1999). Many people with ongoing mental illness have been adversely affected by the casualisation of workplace conditions because, like others who make up the casual workforce, they tend to lack training, consistent work experience and personal contacts in the labour market. Both Terri and Rod described the difficulties, both financial and in worker role identity, which arose from having inconsistent and reduced work hours. This inconsistent work is also described in the literature as precarious work (Ansley, 1999)

I started off like with about 30 to 35 hours a week and then gradually the hours got smaller and smaller, I don't know why that was, but yeah, I think they cut back everybody's hours. (Terri, p. 1)

The job was $\$ 7.50$ an hour and subsidised by WINZ, but the job was fluctuating, like you work three days and then you get sent home, and there wasn't enough to pay the bills. (Rod, p. 1)

Terri and Rod's difficulties with the precarious nature of casual work hours led to them to seek alternative jobs with increased, set work hours. In contrast, Clint talked about how he values the casual nature of his position because the flexible hours have enabled him to gradually increase his work stamina as well as fit in around other life pursuits.

So my stamina wasn't really that great and I had to it earn it up gradually and took about three or six months before I was ready for real work. And then when I was, I couldn't just jump into full- 
time work. I had to do like part-time or casual work. So I joined the company and that suited me perfectly. I joined the company because not only the hours were flexible, but because I can work any days, any time, that I wished to. At first I had to put a good impression so that they like me and they like the work that I do, and then they would agree to the amount of hours that I wish to do. (Clint, p. 1)

In these three scenarios, all the employees were employed on a casual basis. In Terri and Rod's situations, the availability of their work hours was entirely determined by the employer, hence the precarious income stream and lack of regularity in their work schedule led to their resignations. Although Clint was also a casual employee, his ability to mutually negotiate his work hours with his employer, ultimately increased his work satisfaction. Therefore, the informants' lived experience of the casualisation of workplace conditions illuminates the value of what Freire (1972) describes as the co-operative process of problematisation. By employing a problem posing approach within the employee/employer relationship, worker routines are created that address both the employer and employee needs.

\section{Being Acknowledged: Rewards and Promotion}

Seven of the informants stated they had received employer affirmation for their work performance by their employers. For some, this affirmation extended beyond praise to include promotion or job offers. In the following excerpt, Kiri enthusiastically expounds on how her astute decision-making and efforts within the workplace have been acknowledged and rewarded.

Like when I was working in the central area and I always turned up for work everyday, always there early for it and the boss highly recommended us for our hard work. So if you work at something you get rewarded. You make the right decisions you get rewarded and that's how it goes. And you might think that you're making the right decision, and not think anything of it, but they do. You get rewards; it's only all out there. And also counting your blessings, especially when you're been jailed before, because you think of the great things that you've done and achieved since. (Kiri, p.12)

Kiri's excerpt illustrates some of the broader therapeutic benefits of work as stated within the mental health rehabilitation literature. Work is seen to provide a 
distraction from the personal and social limitations a person experiences because they are affected by mental illness. This new hopeful perspective occurs as the person's focus shifts from illness to work issues, thereby enhancing and supporting their recovery process (Gioia, 2005; Marrone \& Golowka, 1999; Mueller \& Wilgosh, 1991; Sundar \& Ochocka, 2004). During their interviews, Joe and Lavina, who had described the additional burden of being recipients of workplace bullying (see p.106) were able to reflect on the positive impact of receiving rare on the job praise.

And the boss who was very straight up, like that's the thing about people that are really straight up you know when they say something they really mean it, and it was after my second week there and he goes, "Lavina, you're doing really well". I was like, "what!" (laugh) and I'm like, "oh thanks" (laugh). (Lavina, p. 4)

../.. I must say he congratulated me on one job the whole time I was on the job. We were at a job and I just finished and he turns around goes to all the workers, "who was the one who did this?" and I turned around, "I did" and he turns around and, "that's a bloody good job mate, excellent job”. (Joe, p. 14)

Not only did this feedback bolster their self-esteem and job satisfaction, for both Joe and Lavina, these unexpected but affirming comments had a much greater positive impact on their job performance than receiving harsh criticism. During their interviews, all the informants detailed with pride, instances where they had received praise for their work performance. In Joe's following excerpt, which concludes his above account of receiving unexpected praise, he illustrates that the positive impact of having one's work performance praised is often profound and long lasting.

The first and only time he congratulated me. I took that really seriously. I really, really appreciated his comment; I really thought, "yeah thank you for that". Then the next day came and more abuse and that went out of the window, "nah fuck he's still the same", but I still never forgot about it though, I still think about it, yeah. (Joe, p. 15)

As occurred for all the informants when they received praise for their work performance, a comment from Joe's foreman boosted Joe's self-esteem and sense of self-efficacy. The motivational aspect of employer praise is encapsulated in 
Kiri's following comment: "When they (the bosses) treat you that great, it makes you want to work even harder for them" (Kiri, p. 4).

Kiri, Rod and Louis' work performance was also acknowledged by job offers and opportunities for promotion. Rod's promotion and job offer was unexpected, with him being somewhat reluctantly promoted to a supervisor position.

../.. I was quite reluctant for them to make me supervisor but in the end they said, "you start so and so day on the day shift," and from then I never looked back. (Rod, p. 3)

During their interviews, all the informants detailed with pride, instances where they had received praise for their work performance. For three informants this acknowledgement of their work performance had extended to job offers and opportunities for promotion. Not only did this affirmation immediately bolster the informants' self-esteem and job satisfaction, the positive impact was often profound and long-lasting. Whether the giver or the recipient of praise, the informants' narratives underscore the mutual employee/employer benefits that positively impact on the workplace culture and production. Creating a humanly enhancing culture aligns with the Freirean (1972) construct of humanisation. This is an emancipatory concept that encapsulates the quest to be more fully human; a quest which is exemplified within this theme. Having their work performance affirmed provided the informants with the opportunity to discover a new sense of self beyond the limitations imposed by their mental illness and prior illness-related offending.

\section{Mastering Work Skills}

Most of the informants described that their return-to-work resulted in gaining a highly valued sense of self-mastery over a range of work skills. Rod, the only person who didn't mention this as part of his experience, had the most extensive work history and returned to a work situation where the tasks were very familiar. The other informants identified mastering work skills as both integral to, and a highly valued component of, the return-to-work experience. The skills that were commented on the most were interpersonal skills: "So the skills that I need are..., I can only say people skills" (Clint, p. 9). 
As exemplified within these excerpts, returning to work provided the informants with valued opportunities to hone their people skills after their socially isolated years of hospitalisation. In addition, they described developing an array of skills that also included intellectual, emotional and physical components. In the following excerpt, Louis describes the work skills that he has both learnt and consolidated, thereby enhancing his future employment prospects.

Reliable, I'm reliable. Well, I'm starting to get a good work history; I think they would give me a good reference. I think I'm a good team player, but also think outside the square if I need to. Not scared to ask questions; better to ask a question than do something wrong and make a major mistake, just a matter of knowing when to know which way is intelligent. I don't know, humble, because I've been in problems. You not only learn to be humble but you actually know what it like is to be there. Some people have never been there, it's like saying to somebody that's been brought up with millions of dollars and one day he lost his all money and he had to go on the benefit, and thought there was nowhere to go. He wouldn't know what to do, yeah. (Louis, p.18)

Within the above excerpt, Louis also highlights how he was able to harness the knowledge that arose from his historical and cultural context, and thereby usefully apply it as lived experience within his consumer advocacy work role. In using his above millionaire example, Louis is critically reflecting on how his difficult life experiences have better equipped him in his work role, than a life of privilege would have. His critical reflection illustrates the Freirean (1972) notion of humanisation, wherein people are called to be self-creating. This creative initiative enables the skills and knowledge that evolve out of challenging situations, to become the basis of transformative action.

For some of the informants, the mastering of work skills has enabled them to learn new tasks within an environment that has meaning for them. In the next extract, Joe describes the extent to which this learned on the job knowledge has enabled both his work performance and his career prospects.

Just knowing I can do it. Knowing I can come in and do it. I know I can do it, I could do this house no trouble; give me the tools I could do this easy. No sweat. I could probably start my own business if I wanted. I reckon I could, I could probably train people up myself. Yep, I could, honestly I could. Like if I had a person who could read and knew a bit about the job and he had 
the charts and knew where the things are supposed to go, easy I could do it, I could come in and do this no trouble. (Joe, p. 16)

Joe's description is particularly poignant in his reference to requiring someone who can read to help him realise his potential. Joe reported that his literacy is very limited, mainly due to his very erratic school attendance. In contrast to many of the informants' negative experiences within the traditional school system (see p.122) work engaged them in meaningful tasks, which in turn increased their motivation for learning. This echoes Freire's (1972) liberatory education construct, wherein the democratised syllabus is centred both on the learners' life experiences as well as their environmental learning needs. As Louis describes below, for most of the informants, this was a very empowering experience as they were able to turn around previous learning failures.

Yeah, like at [workplace] obviously one of the things that comes up all the time is strategic plans and policies and all that sort of stuff, which is exactly what happens at my family meetings but just different context, apart from that it's following a meeting plan. (Louis, p. 13)

According to the 'Psychiatric Rehabilitation' model, one of the characteristics of a skill is that once they have been mastered, they are transferable throughout one's life domains (Anthony et al., 2002). In the above excerpt, Louis describes how the array of skills he mastered in order to participate in strategic planning and policy development at work, have been valued by his whanau at their local huis.

In line with Freire's (1972) notion of humanisation, the informants' creative initiative has enabled some of the skills and knowledge that have evolved out of their previous hardships, to become the basis of their transformative action within the workplace. This action established the foundation whereby seven of the informants described that their return-to-work resulted in mastering a range of work skills. While interpersonal skills were highly prioritised, the informants also described developing an array of work skills that included physical and intellectual components. Work has engaged the informants with tasks and activities that are meaningful to them. Consequently, this meaningful engagement created the learning environment that encouraged the informants' skills mastery and enhanced their employment prospects. This pedagogical 
process mirrors Freire's liberatory education construct, wherein the learnercentred syllabus is concerned with life experiences and environmental requirements.

\section{Fitting in with the Social Scene}

Forming social relationships with work colleagues featured in seven of the informants' narratives. This theme relates to the experience of meeting new people and forging new social relationships with work colleagues. "It's something that happened on the job when I started work. I got to meet new people" (Terri, p. 1). For many of the informants, returning to work provided them with the opportunity to forge new relationships after years of only meeting new people within the confines of their institutional experience. Being able to see themselves through the eyes of colleagues and customers who represented the wider community was a confidence building experience for most of the informants. In the next extract, Kiri's reflections on workplace relationships moves from an initially very broad, and therefore somewhat unrealistic comment, to a meaningful self-reflective critique on the experience of forging interpersonal relationships with work colleagues.

Yeah, I got along with everybody, well you know. So it was good and it worked out ../l.. Yeah, they got to know what sort of person I was see, so, it all worked out ../l.. Well you know, in this world you can't get along with everybody, I mean, you got people that will disagree, you have different opinions, but you can pretty much get along with anybody, yeah. (Kiri, p. 10)

As Kiri pondered further on the experience of being liked, she was able to critically reflect on the positive impact that "being liked" had on her.

It makes you feel good, you know it makes you feel that you've accomplished something, it's a good feeling being liked; it's a good feeling. I think everybody likes to be liked in someway. It encourages you to do even better. (Kiri, p. 12)

Kiri is describing the positive interaction between being liked and included, and being encouraged to achieve. This dynamic reflects the Freirean (1972) notion of humanisation, wherein a humanly enhancing culture encourages selfdetermination and self-efficacy. According to this construct, by taking an active 
role in approaching their workplace relationships based on the principles of social inclusion, the informants are positively shaping the conditions for their very existence.

While the informants valued being socially included in the workplace, this sense of inclusion was not their predominant workplace experience. As previously explored within the overarching theme, 'being set apart' (see p. 99), while Maria valued the experience of establishing new relationships within the corporate world, she also identifies the limitations of these workplace relationships.

I realised that I was reasonably successful in having a superficial relationship (laugh). But at the same time that was frustrating. I mean I'm sure I would have found compassionate people as I did, like for example when I had a bereavement, I found the people working there were really quite human. But at the same time, it's not really the kind of place that you talk about a forensic psychiatric history, so, yeah. (Maria, p.10)

Being involved in tasks and activities within a work environment provides many opportunities for people to build skills and knowledge. In addition, these tasks and activities also engage the worker in forming connecting bonds with other people (Strong, 1998). This finding, from within the literature reviewed for this study, is echoed by Terri: "Just being able to speak to them and you know not get all, what's that word? Mmm.., closed up, you know, being able to speak to them openly, yeah." (Terri, p. 4).

The extent to which the informants formed interpersonal connections with their colleagues varied considerably. As described in the overarching theme, 'being set apart' (see p. 99), all the informants had a circumspect and hesitant approach to their workplace relationships. Most of the informants, however, reported that the work environment provided them with valued opportunities to develop their people skills. For some people these workplace connections also led to social relationships that occurred during breaks, and occasionally, at after work social events. While Maria and Terri expressed some regret about not pursuing closer friendships, none of the informants had formed an out of work friendship with a colleague. The informants' experience is echoed in studies which have identified that work can sometimes increase a person's sense of isolation because of their reduced access to their former social networks, which they have not been able to 
replicate at work (Becker et al., 1998; Bedell et al,, 1998; Strong, 1998). In respect to these findings, all the informants' social networks remained quite separate and apart from their work environment.

\section{Getting On-the-Job Support}

Receiving on the job support after securing employment was valued by all the informants who saw it as being integral to their ongoing job success and satisfaction. Although the informants' experiences varied, this support was provided by bosses and in some instances, colleagues, and included being provided with workplace accommodations. All the informants described receiving highly valued ongoing support from at least one of their bosses. “..//..an understanding boss, even today having an understanding boss is the most important thing to me, yeah, and they were always good" (Kiri, p. 5). Like Kiri, Clint also emphasised how supportive his boss had been: "He was so good to me; he is still good to me" (Clint, p. 9). Clint describes the mutual benefits he seeks to create within the employee/ employer relationship. By proving his work prowess to his employer, Clint's already supportive boss is further encouraged to accommodate Clint's requirement for flexible work hours.

But if you are doing good work, producing good work and you've got something to show, then the bosses are more inclined to give you hours that you wish. (Clint, p. 2)

As in Clint's situation of negotiating workplace flexibility, the effectiveness of worker negotiation crystallised for Maria when she was faced with being given an additional job role, over and above what she had been employed to do. After completing the necessary training and giving this additional role a trial, Maria's angst about the new job tasks was such that she believed she would have to resign. Having previously proved her worth and value, however, gave her negotiation leverage as she explains in the following passage.

I hated it (the new work tasks) and I did it for a couple of weeks but absolutely hated it and so I said, "look I'd rather leave then continue with the work" ../l.. anyway they were great and they said they were really happy with what I was doing with the other part of my work which I was actually employed for, and they didn't want me to leave and that was really nice and so I 
continued with that and I didn't have to do the other work. (Maria, p. 7)

Both Maria and Clint's above excerpts demonstrate that they were involved in an employee/employer relationship that was flexible enough to assign some decision-making rights to the employee in regard to structure of their working week. This is both enabling and empowering for those people who needs have previously been marginalised within the workforce, for example, older workers; people with child caring roles; people with disabilities (Public Service Association (PSA), 2004).

Joe felt supported on a number of different levels by his overall boss who had previously known both Joe and his family. The depth and meaning of this employer support was highlighted for Joe when his boss stuck up for him when he felt at his most vulnerable due to being the victim of workplace gossip: "So the boss stuck up for me a few times. That's pretty cool” (Joe, p. 11).

Like Joe, Maria also described feeling accepted and personally strengthened by being the recipient of employer compassion and support. As in Joe's situation, this support arose from upper level management, rather than the daily grass roots level of supervision.
../.. one of the senior managers, she did have a knowledge of my situation, so she also, I think, was a person with a kind heart. But also she was working from a business point of view as well. They were very happy with my work but I think her compassion came in to it as well, that she was taking that into account which was really nice of her. (Maria, p. 7)

Five informants recounted also receiving support from their work colleagues. As reported within the theme, 'being teased and bullied' (see p. 106), Joe reported the most difficulties in relation to being bullied at work, particularly from his immediate foreman. Conversely, Joe was also able to describe feeling warmly accepted and supported by his two colleagues he worked most closely with. As a social person who enjoys conversation, Joe's following smoko narrative describes the essence of the acceptance, sharing and mutual regard that typified his relationship with his immediate colleagues. 
I don't know..., it just you know..., at smokos he was..., I could sit next to him and like if I ran out of cigarettes I could ask him for one and he wouldn't say, "oh, go buy your own," or "you got paid, go and buy your own", you know. He'd give me a cigarette when I wanted it. And then I come in the next day and I've got cigarettes and I'd offer it to him, "oh, here those cigarettes", and "nah, nah it's sweet, sweet". And we could talk; me and him could talk all day. I knew he was an alcoholic, but he was a good talker; talk to you. (Joe, p. 10)

All the informants described receiving valued ongoing support from at least one of their bosses, with five informants recounting also receiving support from their work colleagues. In addition, five people described being supported by a variety of workplace accommodations that eased their return to work. Job retention, however, continues to be a major challenge facing workers with mental health disabilities, their vocational support workers and the employer (Becker et al., 1998; Bozzer et al., 1999; Dorio, 2004; Michon et al., 2004). Both Maria and Clint's accounts about bolstering their job satisfaction, exemplify the effectiveness of timely employee/employer negotiations that are underpinned by collaboration and shared decision-making. Maria and Clint's experience of effective collaboration is echoed within the innovative work literature that promotes the principles of social partnership within new democratised workplace structures (PSA, 2004). Although an imbalance in power will always underpin the employer/employee relationship, from a Freirean (1972) perspective, using a problem posing approach enables employers and employers to develop mutually beneficial solutions.

\section{Summary}

Within the context of this overarching theme, all the informants described the multi-dimensional complexities of adjusting to a new work environment. While this initial adjustment period is part and parcel of workaday life, for the informants it had special significance after their years of unemployment within a secure care facility. In this regard, the informants' narratives align with recent New Zealand employment literature which highlights that disenfranchised groups, including people with mental illness, are not served well by the confines of the rigid worker day structures and organisational regimes (Lapsley, 2003; Mintrom \& True, 2004). In addition, the mental health vocational literature 
stresses that job retention is a major challenge facing workers with psychiatric disabilities, their vocational support workers and the employer (Becker et al., 1998; Bozzer et al., 1999; Dorio, 2004; Lapsley, 2003; Michon et al., 2004).

Within the context of this overarching theme, the informants' narratives demonstrate that assigning some decision-making rights to the employee in regard to structure of their working week, is enabling and has mutual employee/employer benefits. According to the Freirean (1972) notion of humanisation, by the informants taking an active role in approaching their human and non-human work environment based on principles of social inclusion, they are shaping the very conditions for their existence. Therefore, from a Freirean perspective, the process of emerging from a position of disadvantage within the work environment requires employers and prospective employees to use a problem posing approach in order to develop mutually beneficial solutions. These solutions may include developing individualised workplace accommodations that are able to flex around the individual's needs, thereby enhancing workplace success and satisfaction for people with mental illness in order to address the issue of job retention.

\section{Chapter Summary}

This chapter explored the informants' authentic reflection on the reality of their pre-employment, economic and workplace situations. These reflections are captured within the overarching themes of: 'doing the groundwork'; 'weighing up the economics' and 'fitting in with workaday life'. In placing these experiences into a critical dimension, Freirean (1972) constructs were again employed to inform the data analysis. In adding another dimension to the informants' return-to-work experience, the following chapter seeks to explicate the informants' work experiences in relation to the embodied aspects of self. 


\section{CHAPTER SEVEN: HUMANS AS UNFINISHED AND INCOMPLETE; WITHIN A LIKEWISE UNFINISHED REALITY.}

\section{Chapter Overview}

This is the last of three chapters that seeks to reveal the understandings that have emerged from within the data. The preceding chapters have focused on the informants' experience of the socio-political influences that accompanied their return to work, and the concrete realities of their pre-employment, economic and work situations. Within this chapter, the final collection of overarching themes; 'getting-going on workday mornings'; 'experiencing the physical self at work'; 'self regulating features of illness at work' and 'work as an elixir', seek to explicate the informants' work experiences in relation to the embodied aspects of self. Specifically, this chapter explores the interrelationships between the informants' physicality, emotionality and mental illness, and the relationships that these aspects have with work, both individually and collectively.

As detailed below, under the umbrella of each overarching theme there exist two to four themes that seek to reveal the informants' experiences of their embodied self at work.

\section{Chapter Seven: Themes}

Getting-going on Workday Mornings Medication-induced lethargy and brain fog

Adjusting to new-worker routines.

Experiencing the Physical Self at Work

Fatigue.

Aches, pains and physical changes.

Work tolerance and stamina.

Self-regulating Features of Illness at Work

Managing delusional thoughts.

Discerning symptoms of anxiety versus workplace stress.

Being inattentive and preoccupied.

Formulating management strategies. 
Work as an Elixir

Being bolstered by personal achievement.

Realising career and work aspirations.

Enjoyment at work.

Contributing back to the community.

Applying a Freirean (1972) lens to the informants' experience of their embodied aspects of self, many of the terms used within the preceding two chapters are reintroduced. These constructs include authentic reflection; praxis; transformative action; humanisation; dialogical process conscientisation and critical consciousness. This terminology has previously been outlined in-depth within the methodology chapter, as well as paraphrased in order to provide an orientation to the previous two chapters. Accordingly, the following paragraph provides a brief recap of the Freirean notions that reoccur within this chapter.

The reflection and action cycle of praxis is hinged on authentic reflection, which occurs in the here and now, within the current reality of one's situation. Through engaging in praxis and mutual dialogue, people can move to enacting transformative action upon themselves, and upon the world. Through such action, they move to conscientisation; the heart of liberatory action and education. Conscientisation drives people to break through prevailing societal myths to reach new levels of awareness, and in particular, an awareness of oppression. This socio-political awareness engenders humanisation, wherein people seek to create a humanly enhancing culture. Rather than dwelling on the abstract, Freire (1972) states that conscientisation takes place in real people and in actual social structures. Hence, conscientisation is an ongoing, dynamic process by which people move towards critical consciousness. As people engage in the world as transformers of that world, individual and collective consciousness ensues and this critical consciousness in turn, leads to further action. In and through such action, people cease to see their situation as an enveloping blind alley; instead it emerges as a historical reality susceptible to transformation (Freire, 1972).

In addition, this chapter introduces two new Freirean (1972) concepts; 'a sense of project' and 'liberatory action'. This former construct, 'a sense of project', relates to Freire's perceptions of people as being incomplete and unfinished, and 
therefore they are in the process of becoming. Humans are beings, according to Freire, who move forward and look ahead with 'a sense of project'. The latter Freirean term, 'liberatory action', is action that seeks to transform the social order by promoting participatory and democratised structures, while denouncing authoritarian and alienating systems of organisation. Hence, the governance of liberatory action is dialogical and egalitarian, as personal freedom and the growth of individuals can only occur in mutuality with others.

Within this chapter, the understandings that are revealed about the embodied aspects of self in relation to work are illuminated by the Freirean (1972) constructs outlined above. Freire's constructs provide a critical context in which to view the physical and psychological shifts that are required, in order for the informants to build up his/her workplace stamina and confidence in the face of illness, lassitude and lapsed employment.

\section{Overarching Theme: Getting-Going on Workday Mornings}

Throughout time, the western mental health tradition has seen individuals coping with their severe and complex psychotic illness, with few opportunities to exert personal control in regard to treatment options. Over the past two decades, however, mental health service delivery has had a dramatic shift towards the principles of community integration, which are centred on self-determination and cultural recognition. An outcome of this quest for self-determination is that an increasingly vocal sector of the politically driven psychiatric survivors' movement question whether routine medication regimes are part of the solution, or part of the problem (Deegan, 1992; Lette, 1992). For recipients of forensic psychiatric services care, however, compliance with medication is a requirement. All the informants describe both the costs and the benefits of medication in relation to their work performance. These experiences are explored within the following themes: 'medication induced lethargy and brain fog' and 'adjusting to new worker routines'. In addition, the socio-political influences which impact on the psychiatric medication conundrum are critiqued in terms of the Freirean notions of praxis, mutual dialogue, conscientisation and critical consciousness. 


\section{Medication Induced Lethargy and Brain Fog}

All the informants experienced morning lethargy that made their return-to-work significantly more difficult because it impeded them 'getting-going' in the morning. Seven of them attributed this lethargy largely to the sedating effects of their medication. As Rod and Maria describe, these sedating effects were uniquely confined to the morning, with all the informants experiencing that the lethargy had significantly dissipated by mid to late morning.

My old drug was a bit hard because it made my heart race, but you just carry on and persevere you know, just like working against the wind, you feel it come on but you fight it, the drowsiness ..//.. especially in the mornings. (Rod, p. 5)

I wouldn't be surprised if people bow out of re-entering the work force, or the world in its general sense, because you're so tired. Even though I take my medication about 5:30 pm at night so that I can get up early enough on the next morning, if I listen to my body and did what it wants to do because it is still sedated, I wouldn't get up until 10 o'clock in the morning but I had to learn that I couldn't do that. (Maria, p. 15)

Although the informants' experience of lethargy was significant in all instances, the extent to which this sensation of being sedated impacted on work performance varied from individual to individual. Louis and Joe described a much greater and more concerning degree of debilitation, as they recounted potentially hazardous situations caused by the experience of feeling sedated during the first quarter of the work day.

Oh you're just heavy-headed, lethargic, can't think straight, not to the point where you could actually get to drive or even drive a car to get to a job ..//.. (Louis, p. 1)

I can't see how people can do it (work) with clozapine ${ }^{15}$, 'cause clozapine really sedates you, really makes all your body lazy and it slows you down and your reactions, and like you drop it at $6 \mathrm{pm}$, I take mine at 6pm, and you wake up at 6am, man you get in your car, your driving reactions are quite slow and like I've nearly fallen asleep sometimes on the wheel because I'm so heavily sedated through clozapine, nearly run some girl off the road and stuff like that, yeah, it's really hard, clozapine. (Joe, p. 12)

\footnotetext{
${ }^{15}$ Clozapine (Clozaril) is a 'new generation' antipsychotic medication.
} 
Eventually, most of the informants were able to self-manage the issues relating to the sedating impact of medication on their work performance. Joe, however, who had the most physically challenging work environment, also reported the most difficulty in relation to feeling sedated in the morning. After some frightening near miss events that he attributed to the effects of morning sedation, he eventually quit the job he enjoyed, and he has not sought open employment again. "Yeah, but I really enjoyed it (work). I still enjoy it today...//... I'd like to do it again but I can't, not with medication, not with clozapine, I can't do it, you couldn't, I couldn't do it”' (Joe, p. 3).

Within the mental health arena, the dominance of biochemical solutions has been increasingly challenged over the past decade, with the emergence of new treatment paradigms that are underpinned by the rights of the recipients of psychiatric treatment (Deegan, 1992). In relation to this shift towards selfdetermination, the critical perspective provides insight into the interpretation of the return-to-work phenomenon by examining how the stakeholders are positioned in relation to the key determinants; power and knowledge. Rather than conceptualising a dominant group has implicit power due to professional status, as exemplified in the traditional medical discourse within mental health care, Freire (1972) argues that power is a technique that can be deployed. The transfer of power to people with mental illness requires changes in service delivery philosophy, and also a shift in service user/provider relationships. Accordingly, mental health professionals need to be responsible for ensuring that participation is integral to the service users' treatment plans. This ethos is reflected in Freire's belief that the co-operative process of meaningful dialogue is an important part of the emancipation of disenfranchised people; a sentiment that is echoed by Louis: “I suppose you have to work out your own meds. Everybody's different so you got to have trust in your doctor, and your doctors have got to have trust in you as well" (Louis, p. 2). Therefore, central to managing the disabling impact of sedation caused by medication on work performance, is the relationship between the health practitioner and the client. Louis describes this importance of this collaboration in the following extract.

Plus, there's a lot things that you got to weigh up, you know, especially when you're on medication. Sometimes if you're too 
sedated in the morning, maybe a morning job isn't ideal for you. But then you can also talk to the doctor and try and get something, so that they might say, "well, instead of taking it all at night so you're too sedated in the morning, you can take a bit during the day then". My doctor says, "well, I don't care how you take it as long as you take the prescribed amount". So I take a little bit in the morning, a little bit at lunchtime, a little bit at tea time and then at night time I'm asleep. But I can actually get up in and function in the morning. (Louis, p. 1)

The ambiguities within the psychiatric medication phenomenon are very evident within the informants' narratives. Their descriptions about the negative impact of morning sedation co-exist along with the informants' positive accounts about how their medication has also contributed to their employment success.

Medication has helped me to be stable and have good concentration, and with that I feel I'm a lot more successful and a lot more capable of dealing with problems. I can keep my concentration as well when working. (Clint, p. 3)

Therefore, all the informants in this study perceived that their medication had both aided, and impeded, their return-to-work experience.

To fully position the conundrum of psychiatric medication within a critical hermeneutic interpretation, the informants' subjectivity needs to be considered within the context of their life world, which exists as an expression of their historical and cultural circumstances (van Manen, 1997). In this instance, all the informants had moved from a traditional mental health context of slow mornings and unemployment; situations that accommodated their morning lethargy, into the world of work which requires workers to be attentive at the start of the work day. This role shift moves the issue of morning sedation from the realms of being a normal medication side-effect, into a new environmental context where it now exists as a major impediment to workplace performance.

Although psychiatry is often regarded as a comparatively unexacting science within the healthcare arena, mental health professionals are trained to classify the inherently abstract nature of psychiatric symptomatology in order to determine treatment regimes, often within a biochemical context. Biochemical developments occur within the physical sciences, where proof is based on empirical findings that bring one closer to certain knowledge. In contrast, within 
the social science of hermeneutics, a fundamental premise concerns the relative nature of competing interpretations which leads to probabilistic knowledge (van Manen, 1997). Hence, the rules of evidence are different for the physical sciences, which determine the biochemical developments and prescriptions, and the social sciences in which people with mental illness' lived experience of medication is brought into question. In order for scientific biochemical developments to account for the individual's subjective experience of medication, a radical shift is required in clinical assumptions and procedures. Accordingly, the interpretation of the meaning of the workers' subjective stories about the impact of their medication on their return to work, needs to become the fundamental activity for prescribing and dispensing medication, and as such, coexists along side professional observation and clinical measurement.

\section{Adjusting to New Worker Routines}

The sensation of morning fatigue did not dissipate after the informants' initial return-to-work period. All the informants devised practical methods for managing morning fatigue, which for all of them included taking their medication in the early evening, from five pm to seven pm. Representative of all the informants' reports, the following two excerpts describe the collective experience of an increased need for sleep as part of the return-to-work phenomenon, and the associated need to make adjustments to bedtime routines.

But what I did find quite bothering was the amount of sleep that I was having, but that seem to have change now which is good, yeah. 'Cause like I would sleep you know, I would go to bed early and then wake up really late, honestly my sleep was awful when I was first working. (Terri, p. 10)

Well, because back then I used to be a night owl. I used to watch movies until 9 pm, but yeah, getting out of bed was definitely hard but I still got to work on time you know. I still find it hard today getting out of bed, but I get out eventually. Some of its medication, probably half of it would be medication. (Kiri, p. 7)

In addition to the more obvious changes to bedtime and rest routines, the six informants who remained in work described a variety of personal management strategies they used to lessen the impact of the morning lethargy on their work performance. 
Yeah, a good shower first thing in the morning, yeah, that always helps you out. I've had days when I've gotten up and I've felt tired and had a headache but you have shower, you know, just cut back the hot water and that soon wakes you up. (Louis, p. 2)

In addition to devising personal management strategies, Clint and Louis described being able to negotiate significant workplace accommodations to help them manage the impact of morning lethargy in the workplace.

When I get up in the morning it can be quite tiring after I take my medication...//... the guy that assigns us jobs; he knows what I'm like. Yeah, so he normally gives me about an hour's sleep in the office, just to lie on the couch and gives me a rest, and then he gives me a job later on. (Clint, p. 9)

Well there's no real start time for me, it all depends when the meetings are. Sometimes, I start in the morning, sometimes there's a meeting which could be in the afternoon...// yeah, you know, it's just a matter of knowing what time 'cause obviously I take my meds slightly earlier at night time so I can still get my normal sleep and get up and get going. (Louis, p. 2)

Both Clint and Louis' negotiation skills, as well as the actual workplace accommodations, were integral to their return to work. In this regard, all the informants reported that being in work had enabled them to experience the benefits of increased personal and financial resources. This confidence boost has enabled them to be self-directed in devising and employing an array of work adjustment techniques. In the following self reflective passage, Maria critiques her experience of medication induced lethargy.

I think certainly that there is a sort of absorption factor with the medication. And I don't mean a physical absorption, I mean a sort of psychological absorption that you accept that you want your life to be fuller, you want it to be more developed, you want to be contributing to society. So you kind of move past those physical difficulties and say well this is it. So it's almost like your world absorbs the medication up to a point, but I still find that I need to really push myself to get out of bed in the morning. (Maria, p. 15)

By engaging in this process of critical self-reflection, Maria is able to harness her internalised resources in order to create the change she desires. Her self-reflective process illuminates Freire's (1972) belief that through conscientisation, people are able to identify contradictions in their life world experiences. This 
leads to new levels of awareness, which Freire describes as critical consciousness, and as demonstrated in Maria's experience of medication, this awareness eventually becomes emancipatory knowledge.

\section{Summary}

While the slow mornings of the informants' prior unemployment accommodated their morning lethargy, returning to work has created a new environmental context which brought the issue of getting-going in the morning sharply into question. Although the informants' experience of lethargy was significant in all instances, the extent to which this sensation impacted on work performance varied from individual to individual. All the informants attributed this lethargy, to a greater or lesser extent, to the side effect of their psychiatric medication. In addition, they all concurred that by mid morning this veil of lethargy had usually dramatically lifted. Most of the informants described how building their workplace confidence enabled them to harness their own resources to effectively deal with the impact of morning lethargy. Joe, however, reported that he needed to resign from the position he enjoyed, largely attributing this to the impact of medication sedation on his early morning work performance.

The informants' collective experience is that biochemical solutions do eliminate many of the symptoms of psychosis which can impede their work performance. At the same time, however, the impact of morning sedation can also create significant barriers to realising work goals and career aspirations. Therefore, in accord with the informants' narratives, management of the substantial medication management conundrum essentially hinges on the health practitioner's collaboration and ability to 'hear' the client's lived experience. In this regard, the importance of Freire's (1972) belief in the co-operative process of meaningful dialogue is consistently reflected within the informants' stories about gettinggoing on work day mornings. The informants' narratives highlight the importance of their experience of shared-decision making, as the foundation of medication regimes and workplace accommodations. 
Overarching Theme: Experiencing the Physical Self at Work

This overarching theme is concerned with how, after the slow time spent within a constricted environment, the informants' return-to-work impacted on their physical self. During their interviews, all the informants reported being surprised about the extent to which their return to work had tested their physical condition and stamina. Their experiences of these physical demands will be explored within the following themes: 'fatigue; aches, pains and physical changes' and 'building work stamina'. From a socio-political perspective, in a report about the socio-economic determinates of health, the New Zealand National Health Committee (1998) stresses that the prevailing evidence now demonstrates that social, cultural and economic factors are the most important determinants of good health. In this regard, Freire's critical social theories will be used to illuminate the informants' experiences about their physical self at work. This exploration will include Freire's notions of praxis; a sense of project; authentic reflection; problematisation and the culture of silence.

\section{Fatigue}

All the informants described that their return-to-work was accompanied by varying levels of fatigue. As Rod illustrates below, most of the informants were able to self-manage this fatigue within the workplace, and it abated as their work tolerance increased.

Sometimes the boss would just catch me nodding off, because you sit down and you got to count all these packets and you just tend to nod off and oh boy, yeah, but..//. well you just get up and walk around, yeah. After a while you get used to it and you're able to do your job.. //.. (Rod, p. 6)

In contrast, Joe describes that his extreme fatigue was evident at the end of each day, throughout the entire working week, and that it did not lessen over time.

The tiredness, when you're on medication and stuff. Doing that (work) 5 days a week non stop, it was hard, really hard. At the end of the week, you'd love Friday. I love Fridays, you'd just be laid back and you can relax. (Joe, p. 13) 
In the following narrative, Maria concurs with Joe's sentiments about the interrelationship between work, medication and extreme fatigue.

I found that you have to look after yourself; I found I really could only work a few days a week. Full-time was too much, because you're on this recovery journey, and physically you're tired. I found with my medication I can't, I just can't do it. (Maria, p. 13)

Joe, Maria and Lavina all described the negative impact of work-related stress and overwhelming fatigue, on both their physical and emotional well-being. As Joe illustrates below, his experience of being drained was at the extreme edge of physical and mental fatigue.

Yeah I did get really drained, and part of my illness is if I get too tired, I get too stressed out. It brings on stress an' stuff if you don't have a good night's sleep..//.there must be easier jobs than god damned [trade job] that's for sure. It just drains you aye. Really drains you. (Joe, p. 13)

Within the general job search literature, the fatigue that accompanies starting a new job is well documented (Mintrom \& True, 2004). Hence, there is a degree of validity in normalising fatigue within the context of a new job. Maria and Joe's narratives highlight the additional burdens of medication side effects and the competing demands that accompanied the desired but energy sapping process of their reintegration into the community. "I wouldn't be surprised if people bow out of re-entering the workforce or the world in its general sense, because you're so tired" (Maria, p. 15).

\section{Aches, Pains and Physical Changes}

For Lavina, Joe and Terri, experiencing 'the physical self at work' went beyond work-related fatigue, into the realm of aches, pains and other physical changes.

Well it was funny because it was mostly standing up work..//.. I would walk to the bus stop and my feet would be aching, I would get back home and my feet would be aching, I'd be like, "oh gosh, pain agony”. It wasn't very nice, yeah. (Lavina, p. 7)

In Joe's situation, the pain experienced was related to a specific work task, and it was compounded by the lack of appropriate protective gear. 
I asked the boss for leather gloves and he just said, "nah, nah harden up". He goes, "I started off I was your age..." but nah once you get raw fingers you can't keep going 'cause it just gets worse, you get too sore to hold the tools. (Joe, p. 13)

Returning to work resulted in significant weight changes for Terri and Lavina. In the first instance, Terri described a significant weight gain that concerned her to such a degree, that she decided to resign and seek a job that was centred on physical activity. "I got bored with the job, and also because being in the fast food industry I found that I was eating too much so I don't really want to work around food" (Terri, p. 6). In the second instance, Lavina attributed her dramatic weight loss to an increase in job stress after her workplace was taken over by new ownership. The new owner/operators ran under a totally different management ethos which resulted in Lavina feeling intimidated.

I started realising I had to eat to keep going, because I was so stressed out with the job and the intrusive thoughts and stuff. So I was eating things like muffins with extra cream cheese and butter because I was just fading away to nothing, because of the pressure of the job, and what was happening in my mind, and you know, I was actually withering away. (Lavina, p. 7)

Lavina's daunting experience reflects Freire's (1972) notion, that people are not just limited by the facts and material circumstances of a situation, but also by what Freire (1972) describes as the human situation. In Lavina's case, a change in business ownership led to a major shift in the human situation. With this shift came a culture of bullying that impacted on Lavina's physical and mental wellbeing.

The three excerpts within this theme arose from Terri, Lavina and Joe's experiences of aches, pains and physical changes. These work-related physical consequences significantly contributed to all three informants' decision to resign. According to Freire's (1972) conscientisation process, people are able to gain new levels of awareness through identifying contradictions in their experience. In line with this Freirean thinking, Terri, Lavina and Joe were able to critically reflect on how their prior unsatisfactory work experiences had helped them discern their future work preferences. While Terri had secured another position which involved physical activity, at the time of interview, Lavina was 
contemplating part-time work and Joe was clear he wished to remain working within an affirmative business.

\section{Building Work Stamina}

During their interviews for this research, all the informants reported being surprised about the extent to which their return to work had highlighted their lack of physical stamina. In the following extracts, Lavina and Terri describe how they view their initial work stamina in terms of the increased demands that accompanied their shift into open employment.

../.. it was a bit of a rude awakening because I'd been in hospital for nine years, and out of those nine years, ah for seven to eight years, I did a lot of work in the various [affirmative businesses] which were not really like a regimented job sort of thing. So I did find the hours a bit long, and the work was harder than I expected, and picking up things was also more difficult. (Lavina, p. 1-2)

../.. I had been in hospital and all you had to do was sit around and do nothing and then you would get out into the big wide world again and suddenly you're getting active doing stuff again. Of course it's going to tire you out if you're used to being in a hospital doing nothing all day, you know, of course it's going to tire you out until your body gets used to being active again. (Terri, p. 10)

Lavina and Terri describe their limited work stamina as being a consequence of the years they spent contained within a secure environment. As Lavina highlights above, during this period, she and the other informants were all engaged in work routines within various affirmative businesses. Within the theme, Building work skills and resource (see p. 119); the informants described valuing the activity, structure and remuneration that the affirmative businesses provided. These businesses did not, however, provide a work environment that simulated the physical demands of an open employment job. Hence, all the informants found that their shift to open employment significantly tested their physical endurance. Their collective experience, therefore, aligns with the mental health vocational research that recommends caution when making generalisations between sheltered and open work environments (Anthony et al., 2002; Mueller \& Wilgosh, 1991; Schneider, 1998b). Accordingly, the building of work stamina 
was only able to be fully tested and addressed within the informants' new work situation. This scenario illustrates the Freirean (1972) belief that authentic reflection, which drives one's transformative action, does not occur when abstractly considering past or potential circumstances. Authentic reflection is only concerned with the current reality of one's situation. In line with this Freirean thinking, while important preparatory work occurred during the informants' pre-discharge rehabilitation interventions, their experience of limited work stamina was only able to be fully addressed within their actual open work situation. In the following excerpt, Clint describes how he needed to build up his work stamina by gradually increasing his work hours over a period of months.

So my stamina wasn't really that great and I had to build it up gradually. It took about three or six months before I was ready for real work and then when I was, I couldn't just jump into full-time work I had to do like part-time or casual work. (Clint, p. 1)

For five of the informants, their return to either full-time or part-time work, confirmed for them that they desired part-time employment. This desire was largely driven by them finding full-time work too physically taxing. Some of the informants also commented on the energy expenditure that resulted from workrelated activity such as transport to and from work. In addition, Maria, Joe and Louis talked about the need for reduced work hours in order to accommodate their household tasks. As Maria describes, as well as adjusting to a new work environment, the demands of a new living situation also accompanied the informants' shift back to the community.

I do have physical limitations so I can only work a certain amount. I get so tired, so that's something to learn, and that hopefully I can bring a balance into my life. What happens at the moment, which happens for heaps of people, is that it feels like even though I'm only working part-time, it still often feels like I'm also working at home, you know that kind of feeling. (Maria, p.19)

Maria's intervention was to conserve her energy by negotiating a reduction in hours. In contrast, Rod, Louis, Kiri and Terri all described how they embraced the challenge of building their work stamina. "Well, I like the physical side of it, I wanted to get a bit of exercise, you know, so that helped in that area of being physically active. But it's a very hard job, yeah" (Rod, p. 2). Rod's narrative illustrates Freire's notion that as people continue to modify their environment 
through human activity, as occurred when the informants returned to work, that ever new challenges and tasks will arise. Freire (1972) regards this self-creating as an essential element of being human, and as such, humans are beings who move forward and look ahead with 'a sense of project'.

\section{Summary}

All the informants described their initial surprise at the extent to which their return to work had highlighted their lack of physical stamina. They retrospectively viewed this as an outcome of the 'slow time' that they experienced during their years in a secure care hospital. In addition to the experience of work related-fatigue, three people reported that being in work, and/or specific work tasks had, at times, been detrimental to their physical wellbeing. From a Freirean (1972) perspective, the informants had to make physical and psychological shifts in order to build up their work stamina in the face of aches and pains, and overwhelming fatigue. In line with Freire's notion of problematisation, making these shifts required the informants to perceive their return-to-work physical stamina not as an irredeemable feature, but as merely limiting and therefore challenging. After the informants' initial surprise at their high levels of work-related fatigue, all their narratives demonstrate the action and reflection cycle of praxis which ultimately led to their transformative action. Therefore, they were able to emerge from their often overwhelming sense of exhaustion and reflect on their situation, in order to have a consciously critical confrontation with their problems. The informants were then able to intervene in their own circumstances. Rod, Louis, Clint and Kiri's were all able to hunker down on-the-job and gradually build up their work stamina. Maria successfully negotiated a reduction in her hours of employment. Terri, Joe and Lavina, however, all resigned from their positions. While Terri successfully sought alternative open employment, Joe and Lavina decided to return to the low pressure affirmative business work environment. At the time of interview Lavina was reviewing part-time work options. 
Overarching Theme: Self-Regulating Features of Illness at Work

Despite considerable progress in recent years, within mental health treatment the service users' altered behaviours and experiences that lead to the diagnoses of psychosis are still not well understood. This lack of clarity is related to two very distinct, but interwoven perspectives that are concerned with the recognition and treatment of psychiatric symptoms. As discussed within the overarching theme, 'getting-going on weekday mornings' (see p. 155), the perspective that has held historical dominance is that of the psychiatric diagnostician. Since deinstitutionalisation commenced in New Zealand in the early nineties, the paradigm shift towards the recovery approach has seen the unique perspective of people with mental illness increasingly prevail in their illness management. It is crucial that people with mental illness are actively involved in the health care plans that support their wellness-at-work. This involvement brings with it, however, unique complexities in that the informants are people whose psychosis can present functional changes in the way an individual experiences the world. These complex experiences are revealed within the first three themes: 'discerning symptoms of anxiety versus workplace stress'; 'managing hallucinations and delusional thoughts' and 'being inattentive and preoccupied'. The concluding theme: 'formulating wellness-at-work strategies', explores the internalised processes and practical interventions that the informants employ to self-regulate the impact of both work-related stress and illness-related symptoms. The critical perspective is then explored through Freire's notions of the culture of silence; dialogical process; conscientisation and praxis.

\section{Discerning Symptoms of Anxiety versus Workplace Stress}

Five of the informants described the issue of workplace stress and the subsequent impact on their physical and emotional well-being. The degree to which this workplace stress impacted on people varied considerably. In Louis' situation, feeling these work-related tensions was a reminder to him to employ his stress management techniques. "Stress management you know, stress is normal isn't it; everybody gets it, its how you deal with it" (Louis, p.16). At the other end of the work stress spectrum, Joe describes how his full-time work negatively impacted on his mental state towards the end of each working week. 
Working that hard during all that week, you'd come home and you'd be on the edge mentally..., you would be, I was. I was half way through the week, or at the end of the week, and I was quite mentally ill. You know all that work took it all out of me. (Joe, p. 13)

In contrast, Kiri attributes her episodes of anxiety to her illness, rather than being work-related, and has remedies for the episodes that arise during her working day.

It's just a feeling at first, and then it goes away, anxiety that is..//.. I got medication for that. If I am feeling like that at work I just take some medication prn ${ }^{16} . . /$.. just to get through it. But I find that sleep is good. (Kiri, p.13)

Job stress is both a normal and often an invigorating component of the workaday world. In the first excerpt quoted in this theme, Louis both normalises the experience of workplace stress and advocates for learning stress management techniques. This contrasts with Joe and Kiri's more complex anxiety at work situations, which both required individualised, specialist interventions. Both within the mental health sector and the general community, the stress associated with work has been seen as a historical reason for people with mental illness to avoid employment (Anthony et al., 2002; Marrone \& Golowka, 1999). Upholding this premise, however, illuminates Freire's notion of 'the culture of silence' wherein people with mental illness have historically been excluded from the economic, political and social benefits of work. New mental health recovery principles, however, are centred on social inclusion and unimpeded access to the opportunities and rights of citizenship. Therefore, the historical exclusionary workplace employment practises that were designed to benevolently protect people with mental illness from relapse and re-admission are now increasingly outmoded. As Kiri and Joe's narratives highlight, however, people with mental illness may require individualised stress and anxiety management interventions to be incorporated into their wellness-at-work plans.

\footnotetext{
${ }^{16}$ Medication that is prescribed on a 'take as required' basis in order to alleviate symptoms of illness.
} 
Managing Hallucinations and Delusional Thoughts

Lavina and Kiri were the only informants who described experiencing hallucinations ${ }^{17}$ or delusional thoughts ${ }^{18}$ at work. Below, both women describe the techniques they use to self-manage these thoughts and auditory hallucinations.

I think it was the stress from the job that I started experiencing really bad intrusive, well I don't know if that's the right term, but that's the term I use. Intrusive thoughts, where thoughts just bombard you and come into your mind and just pop up. And it's like when this used to happen to me, a simple thing like adding up a muffin and a coffee, and I'm having an intrusive thought and I'm thinking, hang on, how much is this? So it was a real struggle to do basic things, yeah. (Lavina, p. 2)

Lavina describes how her delusional thoughts suddenly interrupted her work tasks, particularly the more complex cognitive tasks such as calculating. While she could feel bombarded by this occurring, below Lavina describes that she is usually able to "fight" to control the extent to which these thoughts impact on her work.

../.. they were very quick. I would try and control them, or get them out of my head, or stop them from coming in, but most of the time I could fight them off before they came up. But every now and again they would win. They would just like blast me and I'd be like, "I never did that", or, "that never happened". It was really weird. So I experienced that for quite a long time and I thought I would just stick at the job. (Lavina, p.2)

Although the struggle to control her intrusive thoughts is very evident, mostly Lavina describes succeeding in her endeavours to block these thoughts from impacting on her work. Consequently, she decided to stay in the job at that point in time. As highlighted in the following theme, 'formulating wellness-at-work

\footnotetext{
17 Hallucinations are disorders of perception; an individual's sensory experience without appropriate, relevant or adequate sensory stimuli (Miller, Culture, Cruickshank \& Ashton, 1996).

${ }^{18}$ Delusional thoughts are false beliefs held by the individual with absolute conviction, despite being generally discounted by others (Miller et al., 1996).
} 
strategies' (see p. 172); Lavina's eventual decision to resign was actually related to the stress evoked by being the recipient of workplace bullying.

Kiri's episodes of hearing voices ${ }^{19}$ also intruded on her unexpectedly during her working day. In the following extract, she describes how hearing another person's experience of auditory hallucinations became a turning point in her selfawareness.

I've got paranoid schizophrenia, so I will often think I can hear people talking but it's not happening, you know. I actually got more experienced with that through a sixty minutes documentary on a guy over in the States who had schizophrenia, and like he says that he can hear people in the audience saying that he couldn't sing, but no-one was doing that at all. And that's when I sort of thought, "well, that's what happens to me". I can hear people saying "you're a fool" but then that's not people saying it, it's just something I hear, it doesn't necessarily mean that someone said it. That's how I compared it, which made it a bit easier for me and my mental illness, just to compare the same sort of occurrences and it was really good having some sort of insight into someone where he is actually going through the same thing that you go though. (Kiri, p.2)

While Kiri continues to experience these intermittent episodes, having insight into what is occurring enables her to manage these auditory hallucinations. Her description of feeling empowered through the self-awareness that arises from shared peer experience is a process that Freire (1972) refers to as conscientisation. An important step within this process of conscientisation involves identifying contradictions in experience through mutual dialogue. This is exemplified within Kiri's description of hearing a fellow consumer talk about his experience of hearing voices.

\section{Being Inattentive and Preoccupied}

While half the informants described experiencing intermittent periods of having difficulty concentrating at work, only Joe and Lavina described having difficulty actually completing assigned tasks because of feeling preoccupied by their thoughts.

\footnotetext{
${ }^{19}$ Hearing voices: Auditory hallucinations which are a symptom of psychosis (Miller et al., 1996)
} 
Like when you done something wrong, you know, when your mind is somewhere else, and you're thinking of your illness, and your illness is still ticking in your head and then you forget something and then once you forget it, the foreman would just go nuts, sometimes you can't help that because your mind is somewhere else, yeah. (Joe, p.6)

Yeah, yeah simple things that I did day after day could be hard depending on what was happening in my head. (Lavina, p. 3).

For both Joe and Lavina, while these experiences were challenging, they occurred only intermittently, and the severity and frequency lessened over time. The experience of being preoccupied, therefore, impacted much less on their job performance and work satisfaction than the workplace bullying they both experienced (see p. 106), or the morning lethargy that was a daily occurrence for Joe (see p. 155).

\section{Formulating Wellness-at-Work Strategies}

Described within this theme as wellness-at-work strategies, this term encapsulates the practical measures and intrinsic processes that five of the informants used to self-regulate their features of illness at work. In regard to the practical measures, in the following excerpt Clint highlights the importance of medication and supportive relationships in aiding his work performance.

I'm working, achieving, the boss is happy with my work. There's a lot of things that have done me good. The medication has done me good; friendship has done me good; I'm hanging out with my people; I've got support from my church. (Clint, p. 3)

While Clint underscores the importance of external support systems, Kiri stresses her need for self-awareness into how her features of illness may potentially impact on her work performance. As she describes in the following excerpt, this awareness enabled her to harness these symptoms when they unexpectedly arise, thereby minimising the impact of their intrusion on work performance.

It can be quite stressful sometimes. It's not easy for people with mental illness like myself. I have to work that much harder because I think I hear things, but I just carry on. I know when it's someone else, but I know when it's my mental illness, and that sort of knowing makes it easier. If you don't know much about your mental illness, you can't do anything. (Kiri, p. 3) 
In the following excerpt, Kiri expands further on her belief in the importance of having insight into her illness. While she views that insight is developed through professional counselling and education, she stresses the importance of developing self-awareness that is created through a shared experience with others who have had a similar experience.

Yeah, you got to have a lot of insight, you got to../l.. it takes a few years before you actually do get experience with your mental illness and it's not something that happens overnight. It doesn't take two weeks; it takes months, months of counselling and looking into yourself and studying mental illness, and being more aware of yourself. And also being more aware of other people who have mental illness is good, because you're with people who understand how you feel. I don't think people who haven't had mental illness often understood how we feel. Knowing that we had people to get through it with, because that's what saw us through it, with me and my best friend, we went through it together and that builds a really strong relationship. (Kiri, p. 3)

As Kiri described in the preceding theme, Managing delusional thoughts and hallucinations (see p. 170), her self-awareness and self-efficacy have been boosted through being able to mutually reflect on her reality with others who share her experiences. This is the joint process that Freire (1972) refers to as conscientisation. Freire links conscientisation to the relationship between humans and their world, and to 'praxis' as a form of reflection that arises from, and remains indissolubly linked to, active human intervention in reality. In the following excerpt, Louis illuminates the Freirean notion of praxis as he relates the notion of insight into illness with the proactive strategies of stress management.

You get a lot of people that know all those sort of things, but then they can't win when the stress gets on. They can't actually get to the point where they can use them 'cause they have let it go too far. I suppose, you got to be able to read your signs, try to get them early so that you don't have to be hospitalised or whatever. (Louis, p. 16-17)

Louis' assertions reinforce Freire's (1972) notion of praxis, and the importance of reflection and action being performed in a cyclical manner, with timely responsiveness enabling one to illuminate the other in order generate a proactive response to early warning signs. "Yeah, the goal setting; you know, get one; give 
yourself a pat on the back. Then lay on your morals; work on, move on. Or you've got problem solving and stress management" (Louis, p. 16). In identifying the value of having a moral code to "work on, move on", Louis underpins this work ethos with goal setting, problem solving and stress management strategies. While Louis had acquired these tools during formal skills training sessions during his period of hospitalisation, they are transferable skills that he has been able to effectively employ within his workplace experience.

Maria, Lavina and Joe spoke about their fear of getting unwell, relating this in part to both the stress of the job and the pressures involved in maintaining a daily work routine. As described within the theme, 'being teased and bullied' (see p. 106), Lavina and Joe eventually resigned from their full-time positions in order to maintain their mental well-being in the face of job stress which they largely attributed to workplace bullying.

Sometimes I put it on myself how the job ended but then I spring back and I say it was a situation that was a no-win situation and the best thing for my own sanity was to stop working but I do feel a bit guilty every now and again (laugh) because I feel it was a job, but I feel now that hopefully there is a job out there for me that is quite suitable with good owners, you know owners that aren't pushy or too overbearing or what have you. (Lavina, p. 10)

For Lavina, the unsatisfactory experience that led to her resignation became the catalyst for discerning her future workplace preferences. In the following excerpt, Maria also describes her fear of getting unwell because of workplace stress.

I hope I won't get unwell again. I certainly have anxious times, I have stressed times, I have all of that. I feel like I'm pressured all that kind of thing happens to me, but so far I've been lucky to basically maintain my wellness, and so you have to learn to prioritise that as well. (Maria, p. 19)

By critically reflecting on this fear of getting unwell, Maria has been able to tease out that while she has felt the stress and pressures of everyday life as a worker, she has not had a relapse in her symptoms. One of her self-management strategies, however, was to successfully negotiate a reduction in hours to halftime. 
The informants described a range of measures which they use to manage their workplace stress, symptoms of anxiety, and features of illness at work. In addition to having dynamic management plans that are centred on material and practical supports, such as medication and other people, some of the informants described relying on their own resources. These self-management strategies included previously learnt stress management and problem-solving techniques. For two informants, this involved making the difficult decision to resign in order to remove themselves from their stress-evoking situations at work. Some of the informants' also described how their wellness-at-work relied on their ability to critically reflect on their features of illness, such as hearing voices, if they surfaced during their workday. They were then able to employ the knowledge that arose from having insight into their illness to ameliorate the impact of these symptoms on their work performance. The narratives within this theme illuminate Freire's (1972) action and reflection cycle of praxis. Accordingly, the wellness-at-work strategies are hinged on the melding of practical action with self-reflection, in order to bring about timely and proactive in responses to workplace stress and early warning signs.

\section{Summary}

In conceptualising the informants' attempts to self-regulate their illness, it is important to note that the internalised symptoms of psychosis are not only within the person; they also extend to the negotiating of the outer world of work. As Joe, Lavina and Kiri's narratives demonstrate, sometimes they were trying to complete work tasks while they were also attempting to organise thoughts or distinguish reality. The self-regulation of symptoms of anxiety and psychosis appear to be well integrated into most of the informants' wellness-at-work plans. The strategies the informants employed included medication, supportive people and stress management techniques. These practical measures, however, were underpinned by the intrinsic processes of insight into illness and personal validation through shared experiences and mutual dialogue. This illuminates Freire's (1972) belief that the co-operative process of meaningful dialogue is an important part of the emancipation of disenfranchised people. 
Overarching Theme: Work as an Elixir

As highlighted in the preceding themes, for all the informants returning to work was a mixed experience. While many significant workplace barriers continued to co-exist alongside their personal accomplishment, these barriers did not thwart the informants' experience of being bolstered by their return to work. These experiences of work as an elixir are explored within the context of the following four themes: 'being bolstered by personal accomplishment'; 'realising career and work aspirations'; 'enjoyment at work' and 'contributing back to the community'. In revealing the informants' experiences of accomplishment, enjoyment and contribution from a critical perspective, a Freirean lens is applied with particular emphasis on humanisation, transformative action, praxis, a sense of project and liberatory action.

\section{Being Bolstered by Personal Accomplishment}

All the informants described testing their skills within the workplace, which led to the feeling of being bolstered by his or her personal accomplishment. As Joe exemplifies in the following excerpt, this sense of self-efficacy was internally driven and therefore not dependant on praise or encouragement from bosses or colleagues.

It was like when I used to do jobs, looked at my finished products, especially when I finished a whole house or a whole factory and you look back at your job and you just look at the finished product and it looks new and it's primo..//. that was my favourite, actually seeing it at the end. (Joe, p. 5)

In Joe's description, he illustrates how he is bolstered by achieving a prized end product as the fruit of his labours. In addition to this direct link between specific personal endeavour and achievement, all the informants described how their overall return-to-work experience boosted, to greater and lesser extents, their sense of personal accomplishment. This bolstering of the informants' selfefficacy and self-confidence is exemplified in Joe and Kiri's reflections on his/her overall return-to-work experience.

Just knowing I can do it that was all, it gives you a bit of pride when you know you can do something. It makes you feel a bit better, yeah, that's what I think. Makes me feel better about 
myself knowing I've actually achieved something and I know I can go in and do it, yeah, that stands out for me. (Joe, p. 17)

It's a big accomplishment. I feel good about myself, I have my family. I'm pleased with the change. I think everyone is pleased with the change. (Kiri, p. 14)

Returning to work exposed all the informants to an array of challenges and personal opportunities. Having the opportunity to test his or her skills within meaningful work resulted in all the informants experiencing an internalised sense of self-satisfaction. From a Freirean (1972) perspective, this experience of 'being bolstered by personal achievement' can be illuminated by his notion of transformative action. In line with this thinking, the informants' critical reflection on their workplace achievements has enabled them to start transforming their lives in a new found spirit of hope.

\section{Realising Career and Work Aspirations}

All the informants described how their return-to-work experience led to, or had the potential to lead to, realising their work and/or career aspirations. In the following excerpt, Louis summarises his return-to-work experience. After talking about his initial desire to work, Louis describes discerning his career aspirations and then mobilising these in a graded, step-wise fashion.

I always wanted to get to work at some stage. I think that I was capable of working in something, just a matter of finding a job or a career that I wanted to do. I could never see myself as a labourer sort of thing, so it was a matter of just waiting for an opportunity to arise and go from there. But the actual opportunity was the organisation I'm working for. I think I might have helped myself into that job 'cause I used to go to meetings voluntarily, and from these things just progressed and I started doing parttime work and now there's too much work, I can't do it all. (Louis, p. 1)

Being able to gradually build up his work stamina during his graduated return-towork, has enabled Louis to effectively learn and integrate his new work skills as well as gain a new sense of self beyond the impact of his illness. "I think I can see the..., I suppose the light at the end of the tunnel, you know, I think I can go quite far in that organisation; I'm interested in what I am doing” (Louis, p. 10). The high level of success and satisfaction Louis experiences in his current job 
has led to his strong commitment to stay and grow within that work environment. "Yeah, not that I wouldn't work in another environment, but it just so happens that I got everything in my organisation. I'm happy with it anyway” (Louis, p. 8).

In contrast to Louis' desire to continue to progress his career within his current employment, Maria, Clint and Terri all describe seeing their initial return to work position as just a stepping stone to future employment prospects. "The more work experience I have, the better I'll be in another job. I want to get another job (Terri, p. 13)". As Maria and Clint describe in the following excerpts, they envisage that their initial foray into work will eventually lead to future work ventures in which they are more likely to realise their preferred career directions and work aspirations.

I also believed that work is important, preferably work that you like to do. So in going back to the corporation, I always knew it was just a stepping stone. I knew I wouldn't stay there the whole time, but I hoped that it would help me to then move on to something that I really was interested in. (Maria, p. 17-18)

My wish is to earn good money and for opportunities to open up..//.. If something turns up, and I'm allowed to pursue it, then it's to my advantage to see if it's the kind of work that I'm really, really interested in. (Clint, p. 2)

The reality of the ongoing interplay between individuals and their world constitutes the Freirean (1972) notion of humanisation. Since humans do not exist apart from the world, the movement towards the informants' work and career goals must begin with the relationship they have with their world. As the informants have continued to modify their work environment through their human activity, for some, new work aspirations have arisen and potential career directions have evolved.

\section{Enjoyment at Work}

Moving beyond expressions of mere workplace satisfaction, three of the informants repeatedly described enjoying their work. For Louis and Kiri, this experience aligned with their positive return-to-work experience in which they both felt high levels of both success and satisfaction. "Oh well, only I am 
enjoying it, and want to carry on doing it" (Louis, p.19). From a more tangible perspective, Kiri's experience of enjoying work was uniquely weighted towards appreciating her smart work uniform and the aesthetics of her work environment. "The [work premises] was an awesome experience. I got to see the great big stadium and the corporate boxes upstairs and the buffets, you know, done up for everybody and yeah, it was quite amazing” (Kiri, p. 6). In contrast to Louis and Kiri's almost wholly positive experiences, Joe's return-to-work had been a mixed experience. In reflecting on why he eventually resigned from a career he'd enjoyed, Joe attributed this to his medication-induced morning lethargy and being a recipient of workplace bullying. During his interview, however, Joe heavily underscored how much he enjoyed his actual work tasks and seeing the end result of his labours. "I really enjoyed my job .../.. I really liked to finish a product at the end. That I really, really enjoyed, I really enjoyed doing that" (Joe, p. 1).

From a Freirean (1972) perspective, in transforming their former world of unemployment and social isolation to a workaday world, the informants are shaping the very conditions for their existence and their life. For Louis, Kiri and Joe, this role shift saw their accomplishments and workplace appreciation being highlighted by an overall sense of enjoyment.

\section{Contributing Back to the Community}

On more than one occasion within their interviews, both Maria and Louis described the importance of contributing back to the community. For Louis, this belief existed as both a driving personal philosophy and a cultural workplace value. Louis describes the closeness of the service providers and users as an underlying value of working in a Kaupapa Maori service agency. "Well, one the organisation is a Maori Kaupapa so it's not a big organisation..//. You know we are really close people, and easy to talk to" (Louis, p. 7). Being employed by a Kaupapa service, has led to Louis embracing his role as a mentor for others, particularly those who have walked a similar path within forensic services.

Well, I'm a role model for others. You know, if I can do it; they can do it. It's not easy you know, there's a lot of hard work that's 
got to go into it. You know, reading, a lot of hard work, time management that sort of stuff. (Louis, p. 5)

Like Louis, Maria also has strong values about making a community contribution, which drive both her workplace participation and her work aspirations.

I had a belief that I should be working..//.. One of the big things for me is about contributing back to society, so I felt that it wasn't just a should, it was something that I wanted to do. (Maria, p. 4)

Both Louis and Maria describe how their own transformative experiences have become their catalyst for being a role model for others who share their journey. The motivation behind the embracing of this transformative role is mirrored in Freire's (1972) reflections following a working sojourn to the USA in 1967. Speaking as a person of the third world, he argued that the third world is not a geographical concept, but essentially a socio-political character. According to Freire's thinking, people who are alienated within any society are more able to be moved to transformative action by others who have previously been able to emerge from the same marginalised position. In further examining the notion of being a role model for others' transformative action, it is useful to look at the debate surrounding the western construct of empowerment versus the Freirean notion of liberatory action. In this debate, Freire was sceptical about the ability of western thinking to conceptualise empowerment as a social act, instead of an individual characteristic. Even when an individual feels the freest, Freire argues, if it is not a feeling that is shared with his or her compatriots, then the individual is realising only an individualist attitude toward empowerment. In contrast, Louis and Maria's advocacy position illuminates the Freirean notion of liberatory action. This is a direction which sees an individual utilising his or her recent freedom to help others to be free by transforming society, thereby leading to true collective emancipation.

\section{Summary}

Returning to work exposed all the informants to a mixed array of challenges and personal opportunities. Despite significant workplace barriers, all the informants had the opportunity to test their skills within meaningful work activity. For all 
the informants, mastering work skills led to a strong sense of self-satisfaction. This satisfaction initially arose from the accomplishment of specific work tasks, which then generated the informants' generalised experience of being bolstered by returning to work. Despite Joe and Lavina experiencing workplace setbacks that eventually led to their resignations, all the informants concurred that work had bolstered their self-efficacy and self-esteem. From a Freirean (1972) perspective, the informants are emerging from their former set-apart world of secured care, and intervening in their historical reality by embracing the workaday world. Their experiences of work as an elixir can therefore be viewed in terms of Freire's humanisation, whereby they are changing their world, and changing themselves, by responding to the call to be self-creating.

\section{Chapter Summary}

In this, the last of the three findings chapters, the focus was on the interrelationships between the informants' physicality, emotionality and mental illness, and the relationships that these aspects have with work. From the critical perspective, Freirean theory was once again used to inform the final collection of overarching themes; 'getting-going on workday mornings'; 'experiencing the physical self at work'; 'self-regulating features of illness at work' and 'work as an elixir'. The preceding two chapters have focused on the informants' experience of the socio-political influences that accompanied their return-towork, and the concrete realities of their pre-employment, economic and work situations. In focusing on the informants' experience of their embodied aspects of self, this chapter sought to reveal another dimension of the informants' returnto-work experience. The following 'discussion and conclusions' chapter reviews and summarises the three findings chapters, which are chapters five, six and seven. The findings are then integrated into eight recommendations that have the potential to inform mental health rehabilitation practise, from the forensic psychiatry and vocational rehabilitation perspectives. 


\section{CHAPTER EIGHT: DISCUSSIONS AND CONCLUSIONS}

\section{Chapter Overview}

The purpose of this, the last chapter of the thesis, is to summarise the findings revealed in each of the three data chapters, and then integrate these findings into forensic and vocational rehabilitation practise and research. Initially, each of the three findings chapters are reviewed and summarised. These paragraphs seek to emphasise the eleven overarching themes which are italicised in order to highlight them within the text. Following these summaries, the Freirean notions that were used to explicate the return-to-work experience from the critical perspective are reviewed. Again italics are used to highlight these constructs in order to demonstrate how they interrelate with the return-to-work phenomenon under study. Within the next section, the implications for mental health and vocational rehabilitation practise are described, with these recommendations being related to both the data and Freirean critical theory. These eight implications for practise are concerned with rehabilitation and vocational interventions, and therefore extend to influencing employment practises within a socio-political context. In concluding this chapter the educational aspects are described, and the limits of this study and further research directions are identified.

\section{Returning to Work: Summary of the Findings}

\section{Chapter Five: Liberatory Quest: From Alienation to Inclusion}

In the first findings chapter, 'Liberatory quest, from alienation to inclusion', the Freirean notions captured in this heading help reveal the understandings that have emerged from the data. The first four overarching themes, Re-entering the world, Fortitude, Being set apart and Redeeming oneself are all concerned with the informants' liberatory quest, which both supported, and was supported by, their return-to-work journey. Through this quest, they were able address their unemployment and social isolation, and thereby experience the sense of inclusion that accompanies being in a worker role. 
Securing employment accentuated the informants' 'recovery' outcomes, as this is the juncture where they were seen to re-enter the world. For both the person and their family or whanau, being in a worker role has underscored the individual's wellness. This new role has also served to create a perception of distance from the devastation created by the informants' illness and prior illness-related offending. Since deinstitutionalisation of the New Zealand mental health system commenced in the early nineties, however, the general community has been fuelled by sensationalistic reports within the media. This misleading reporting has combined with, and contributed to, some of the prejudicial attitudes and negative stereotyping. This stigmatisation is poignantly conveyed in the informants' narratives of being set apart. For two of the informants, this experience was at the extreme end. The bullying included both verbal abuse and physically intimidation, which contributed to both of them resigning and choosing to work within a supportive affirmative business. Most of the informants' narratives, however, demonstrate that with personal fortitude and work as a primary instigator, they have been able to address their marginalised position and ameliorate, to varying degrees, their sense of social isolation.

For all the informants, their fear of potential exclusion because of having a history of mental illness and illness-related offending has seen them adopt a hesitant and circumspect approach to their workplace relationships. In regard to divulging information about their mental illness; when, what and to whom to disclose was regarded by most of the informants as the most challenging issue facing people with mental illness who are entering the open workforce. This conundrum underscores the challenges the informants faced when reconciling their prior history with their new worker role. Redeeming oneself refers to the informants' felt need to reconcile aspects of their identity that are rooted both within and outside of the forensic psychiatric institution. Consequently, their experience of redemption was sharply brought into question with the shift from their set apart world, to living and working into the community. The informants' experiences that have been revealed in chapter five illuminate that returning to work was a liberatory quest which presented both challenges and creative possibilities for all the informants. 
Chapter Six: Authentic Reflection; Being Grounded in the Here and Now, within the Reality of the Situation

'Authentic reflection; being grounded in the here and now, within the reality of the situation' is the Freirean thinking that constitutes the title for the second data chapter. This chapter sought to explore the more concretised experiences that are encapsulated within the overarching themes of: Doing the Groundwork; Weighing up the Economics and Fitting in with Workaday Life. Hence, chapter six was concerned with how the informants' experienced the actual work-related situations in which they found themselves immersed. In addition to focusing on the pre-employment, economic and workplace circumstances, this chapter also sought to reveal the informants' responses to the new challenges and tasks that continued to evolve from within these return-to-work situations.

For all of the informants, re-entering the open work environment required them to engage in preparatory groundwork. Doing the groundwork included building skills and resources within affirmative business opportunities, and/or drawing on training and education opportunities. In addition to this learning and skills building preparation, most of the informants had their re-entry to work assisted by the proactive interventions of others, including: friends; family; forensic staff, vocational practitioners and prospective employers. This contrasted markedly with two informants' autonomous job seeking endeavours that resulted in them securing positions with relative ease. Whether the informants eventually secured open employment autonomously, or with help from others, doing the preparatory groundwork awakened in all of them the expectation of change in their position of long-term unemployment. Doing the groundwork, therefore, instigated their transformative role shift from unemployed beneficiary to worker.

The informants' return-to-work was typified by low hourly pay rates and parttime hours. In most situations, the wages received resulted in only a minimal increase in their available income after work-related costs had been deducted. When weighing-up the economics of work, however, regaining a sense of economic freedom, albeit from a very limited financial pool, was a highly valued return-to-work outcome for all the informants. Although everyone acknowledged that their wages needed to be carefully budgeted, this additional income did 
enable most of the informants to achieve valued goals, such as improvements in housing, which had previously been beyond their means.

All the informants described the multidimensional complexities of adjusting to a new work environment. While this initial adjustment period is part and parcel of workaday life, fitting in with workplace norms had a special significance for the informants after their years of unemployment living in a secure care hospital. The informants' experiences are echoed in recent New Zealand employment literature. These worker-centred initiatives argue that people, who are marginalised in the labour market, including people with mental illness, are not served well by the confines of the rigid workaday structures (Lapsley, 2003; Mintrom \& True, 2004). The informants' experiences highlight the value of assigning some decision-making rights to the employee in regard to the structure of their working week. This practice is solutions-focused and has mutual employee/employer benefits. When the informants were able to be actively involved in structuring their working week, they all reported an increased sense of workplace success and satisfaction. These solutions included developing individualised workplace accommodations, such as part-time flexible hours, which were able to flex around the informants' needs and the employer requirements.

\section{Chapter Seven: Humans as Unfinished and Incomplete, within a Likewise Unfinished Reality}

'Humans as unfinished and incomplete, within a likewise unfinished reality' is a Freirean (1972) phrase that encapsulates his notion of humanisation. This phrase is the title of the third data chapter which explores the interrelationships between the informants' physicality, emotionality and mental illness, and the relationships that these aspects have with work. The overarching themes in chapter seven: Getting going on workday mornings; Experiencing the physical self at work; Self regulating features of illness at work and Work as an elixir, sought to explicate the informants' work experiences in relation to internalised aspects of self.

All the informants described their initial surprise at the extent to which their return-to-work had highlighted their morning lethargy and lack of physical stamina. The 'slow time' of the informants' prior unemployment and secure-care 
hospitalisation had both accommodated, and contributed to, their decreased physical stamina and difficulties in getting-going on workday mornings. Therefore, returning to work had created a new environmental context that brought these issues sharply into focus. Although the informants' experience of morning lethargy and reduced physical stamina was significant in all instances, the extent to which these issues impacted on work performance varied from individual to individual. In addition to the experience of work related fatigue, three of the informants also reported that being in work, and/or specific work tasks had, at times, been detrimental to their physical well-being.

All the informants, to a greater and lesser extent, attributed their morning lethargy to the side-effect of their psychiatric medication. Their collective experience is that biochemical interventions do eliminate many of the symptoms of psychosis that can impede work performance. At the same time, however, the impact of morning sedation can also create significant barriers to realising work goals and career aspirations. The informants' narratives highlighted the importance of their lived experience of morning lethargy being the initiator of shared decision-making. This collaboration provided an effective foundation for discerning the informants' medication regimes and workplace accommodations. After the informants' initial surprise at their high levels of work-related fatigue, most of them reported slowly building their work stamina. One person successfully managed the issue by negotiating a reduction in hours. Two of the informants, however, resigned from their full-time positions and returned to work in a supportive Affirmative Business. Both informants acknowledged that their limited work stamina had contributed to the decision to resign from their fulltime positions. They also attributed their resignations to the workplace bullying they had both been subjected to.

In conceptualising the informants' attempts to self regulate their features-ofillness it is of significance to note that while this theme was touched on by seven of the informants, it was the theme that received the least overall comment. This outcome is mirrored in the literature reviewed for this study. The authors concur that the impact of stigma and discrimination impedes the return-to-work process to a much greater extent than an individual's psychotic illness (Anthony, Cohen, 
Farkas \& Gagne, 2002; Bybee, Mowbray \& McCrohan, 1996; Dorio, 2004; Garske, 1999). Most of the informants described experiencing symptoms of anxiety and workplace stress. Three of the informants also described trying to complete work tasks, while they were attempting to organise thoughts or distinguish reality. The self-regulation of symptoms of anxiety and psychosis, however, appear to be well integrated into most of the informants' wellness-atwork plans. These strategies include medication, supportive people and stress management techniques. In addition, these practical measures were underpinned by the intrinsic processes of insight-into-illness and personal validation through shared experiences and mutual dialogue.

Returning to work exposed all the informants to a mixed array of challenges and personal opportunities. Despite significant workplace barriers that eventually led to two people resigning, all the informants had the opportunity to test their skills within meaningful work activity. Accomplishing these newly acquired work skills resulted in all the informants experiencing a strong sense of satisfaction; work as an elixir. This satisfaction initially arose from the accomplishment of specific work tasks, which then generated a generalised sense of enhanced selfefficacy and self-esteem. To place this theme in a broader context within the overall analysis; the number of times that work as an elixir was revealed within the text, matched the number of times the theme persevering against the odds was explicated. Although existing at opposite ends of the barriers versus accomplishment spectrum, the frequency match between these opposing experiences reflects the mixed nature of the informants' return-to-work journey.

\section{The Relevance of the Critical Perspective}

Freire's (1972) critical social theory acknowledges the lived experiences of people while advocating and working toward the ultimate goal of emancipatory knowledge. By applying a Freirean lens to the informants' experiences, the following paragraphs summarise the socio-political influences on their return-towork endeavours. The Freirean constructs have been italicised, and the interrelationships between these different notions have been explained. 
Many people who are affected by mental illness are excluded from citizenship rights due to the stigma of mental illness and prior illness-related offending (Joe, 2003; Taylor \& Gunn, 1999). Hence, it is not surprising that all the informants had the lived experienced of being set apart in the workplace. Many of the informants' narratives, however, demonstrate that with work as a primary instigator, they are people who have been able to emerge from their situations of alienation by casting aside the culture of silence that has historically and culturally held their potential submerged. In situations of alienation, Freire (1972) acknowledges people may be impaired in their use of power. Freirean theory has highlighted that people who are intervening in their own reality of unemployment and prior secure-care hospitalisation, often need help from others in order to smooth their transition back into the workforce. These shared decision making principles are mirrored in Freire's notions of meaningful dialogue, which exists within the action/reflection cycle of praxis. This construct, which is central to Freirean thinking, is also illustrated in the construction of wellness-at-work plans. Praxis in this instance, involves reflection and action being performed in a cyclical manner; one step constantly illuminating the other, in order generate a proactive response to workplace stress and early warning signs.

Work is an activity that promotes social inclusion (Freire, 1991; Mitchell, Betts \& Epling, 2002). Therefore, the social inclusion of individuals with mental illness into work may possibly ameliorate the damaging mental health effects of social exclusion (Mitchell et al., 2002). According to Freire's liberatory action construct, the task of the disenfranchised is to liberate themselves and, in the process, liberate society from their prejudicial attitudes. In taking an active role in transforming their work environment based on principles of inclusion, people with mental illness are engaging in transformative action; shaping the very conditions for their existence and their life. Freire (1972) describes the process of people seeing themselves as being central in determining their own destiny, which is integral to the recovery process, as conscientisation. Assuming a worker status elevated the informants to a position that enabled them to see beyond their historically limited horizons. Therefore, they were able to regard themselves as being central to determining their own destiny. Freire (1972) purports that through conscientisation, people are able to identify contradictions 
in their life world experiences. Then, through critical reflection and dialogue, this awareness eventually becomes the emancipatory knowledge of critical consciousness. With this comes a new view of reality and a founded hope for freedom (Freire, 2004).

From a Freirean (1972) perspective, the process of emerging from a position of disadvantage within the work environment requires employers and prospective employees to use a problem posing approach in order to develop mutually beneficial solutions. These solutions may include developing individualised workplace accommodations that are able to flex around the employee needs, and thereby enhance their workplace potential. As the informants have continued to modify their work environment through their human activity, new work goals have arisen and potential career directions have evolved. This dynamic reflects the notion of humanisation, wherein a humanly enhancing culture encourages self-determination and self-efficacy. Accordingly, the Freirean principles of humanisation provide a context with which to view the informants as people who are emerging from their former set apart world of secured care.

Consistent with the notion of humanisation is the ongoing interplay between individuals and their world. Since humans do not exist apart from the world, the movement towards the informants' work and career goals must begin with the relationship they have with their world. This scenario illustrates the Freirean (1972) belief that authentic reflection does not occur when abstractly considering past or potential circumstances. Instead, it is reflection that occurs in the here and now, within the reality of ones current situation. In line with this thinking, while important preparatory work occurred during the informants' pre-discharge rehabilitation interventions, their experience of themselves in a worker role was only able to be fully addressed within their actual open employment situation. In this regard, returning to work has been an important catalyst for the all informants, who have illuminated the Freirean notion of a sense of project, by moving forward and looking ahead to work aspirations and career opportunities. 
Forensic Vocational Rehabilitation: Implications for Practise

The areas that were highlighted both within the informants' narratives, and also within the follow-up member check session are individually explored in the subsequent eight subheadings, which outline the implications for practise. Freire's constructs are again used to add critical depth to these recommendations, with the informants' narratives highlighting the lived experience that underpins these practise insights. While these implications are focused on the area of vocational practise from a forensic psychiatry perspective, they also have applications to the broader area of mental health vocational practise.

\section{Vocation as an Integral Part of Forensic Psychiatry Rehabilitation Practise}

From rehabilitative, vocational and occupational therapy perspectives; there is a paucity of research from the forensic psychiatry perspective (Couldrick \& Aldred, 2003; Lindqvist \& Skipworth, 2000; Lloyd, 1995; Tracey, 2000). Many of the informants' experiences align with the international literature reviewed for this thesis. Therefore, both these sources provide directions and insights to guide vocational rehabilitation practise from a forensic psychiatry perspective. While the following seven practise implications explore specific aspects of vocational practise in more depth, this initial heading aims to position vocation as an important component within forensic psychiatry rehabilitation endeavours.

Along with the informants drive to achieve their work goals, comes an initial move away from being solely reliant on District Health Board (DHB) Forensic services. Dedicated vocational practitioners, career advisors and job brokers from both other DHB and Non Government Organisations (NGOs), work intensively with people who are job-seeking in order to enhance their employment outcomes. The complexities involved in the informants' shift to a job seeker role, however, require astute and timely follow-up support from the forensic staff. They are the professionals involved with the return-to-work process, who are at the coal face of managing both risk factors and ensuring the clients' mental health and general well-being. North American research has identified mental health staff attitudes as a barrier to people with mental health disabilities seeking employment (Anthony et al., 2002; Baron \& Granger, 1997; Marrone \& Golowska, 1999; 
Pratt et al, 1998). The informants, however, reported that the forensic staff involved in their care were supportive and encouraging of their return-to-work. In support of this view, Louis also reflected on how the treatment and rehabilitation interventions, which occurred during his stay in a forensic hospital, significantly contributed to him securing a job following his discharge. "Yeah, well suddenly the work that was put in from people from the Forensic service... You know, they put a lot of work into it, not just my key worker, but everybody, yeah" (Louis, p. 15).

The informants' narratives highlight the importance of receiving astute, timely feedback and encouragement, and being taught critical skills that they were able to successfully transfer into the work environment. Their experiences also mirror the research-based recommendation that promotes the importance of acquiring confidence and knowledge in modern-day job seeking skills (Gioia; 2005; Killeen \& O'Day, 2004; Krupa, 2004; Torrey et al., 1998). In addition to assistance being an individual prescription based on the informants' level of skill, the degree of support required is dictated by the specific requirements of each employment environment. While two people secured their work independently, the other informants described how their re-entry to work was assisted by professionals, from both forensic psychiatry and vocational support services. From a Freirean (1972) perspective, people who are marginalised within a society become submerged in their situation and, as long as they remain so submerged, they cannot be actively engaged in the struggle for their own liberation. As the informants are people who are historically disadvantaged within the employment sector, they require access to the skills and resources possessed by: family and whanau; vocational specialists; forensic rehabilitation practitioners and clinicians in order to seek, secure and keep employment.

\section{Doing the Ground Work}

For all of the informants, re-entering the open work environment required them to engage in preparatory groundwork. This included building skills and resources within affirmative business opportunities (see p.119). During their period of secure-care hospitalisation, all the informants had been involved in one or more affirmative consumer-driven work projects. In all cases, this participation was 
regarded by the informants as a positive precursor to open employment. These work projects provide initial respite wherein people living in forensic inpatient units able to hunker down within a supportive, low pressure work environment. The structure, socialisation and being 'kept busy' was seen by the informants, as contributing to their healing. In addition, this work helped to ameliorate the 'slow time' associated with secure-care hospitalisation. In addition, these affirmative businesses provided the informants with skill and resource building opportunities which ultimately assisted their open employment work endeavours.

The European experience of 'Social Firms' and the research evidence from the 'Village Model', as described in the literature review (see p. 16), have demonstrated the value of combining small transitional employment opportunities with supported employment in bridging the gap between work and unemployment (Chandler et al., 1997; Krupa et al., 2000; McCrum, 1997; Schneider, 1998b). These consumer enterprises offer alternatives to the traditional sheltered workshops and are a viable response to service users' demands for better quality services with more participatory and democratised structures. As the informants' narratives have demonstrated, these opportunities have also offered a stepping-stone to real work, for real pay (Krupa et al., 2000). "Working in an Affirmative Business. Yeah, it got me back into doing something for a pay. Yeah, it gave me an incentive to go out and get work again (Terri, p. 2)". The informants' return-to-work experiences concur with the research, however, which suggests that differences between sheltered and open employment environments, in both requirements and opportunities, requires a cautionary approach to making generalisations about worker performance between the two setting types (Mueller \& Wilgosh, 1991; Schneider, 1998b).

For some of the informants, doing the groundwork also included drawing on training and education opportunities. The two people who had previously achieved academically, both viewed that re-visiting formal education opportunities had assisted their pathway back into employment. Whether within an affirmative business, or drawing on past learning and work situations, learning on-the-job was the preferred training method for the other informants, who had not achieved academically within the traditional school system. This preferred 
method of learning reflects the Freirean (1972) experience of liberatory education wherein the syllabus is centred on the learner's personal experience and pressing social needs. Accordingly, most of the informants preferred on-thejob learning that employed a multitude of teaching modalities, including visual demonstration and hands-on practise.

\section{Enhancing Worker's Success and Satisfaction}

Receiving on-the-job support after securing employment was valued by all the informants, who saw it as being integral to their ongoing job success and satisfaction. A fundamental tenet of the psychiatric rehabilitation approach is that rehabilitation endeavours aim to help people with psychiatric disabilities to both respond to the demands of the environment (success), and the internal desires of the individual (satisfaction) (Anthony et al., 2002). Research conducted by Anthony et al. (2002) demonstrates that feeling successful and satisfied within an environmental role, such as a worker role, leads to people staying in that role. Job retention, however, is arguably the most significant challenge facing workers with mental health disabilities, their vocational support workers and the employer (Becker et al., 1998; Bozzer et al., 1999; Dorio, 2004; Michon et al., 2004; Lapsley, 2003).

Many of the informants' reported having their job satisfaction boosted by timely, collaborative employee/employer negotiations. These experiences align with recent New Zealand disability-in-employment literature. This literature highlights that groups previously marginalised within employment, including people with mental illness, are not served well by the confines of the rigid workplace structures (Lapsley, 2003; Mintrom \& True, 2004). Increasingly, much of the general work literature is embracing the principles of social partnership in the workplace, encouraging worker/employer collaboration and shared decision-making under new democratised workplace structures (Minister for Disability Issues, 2001; Public Service Association, 2004).

The informants' accounts demonstrate that assigning some decision-making rights to the employee in regard to the structure of their working week is enabling for those people who needs have previously been marginalised within 
the workforce. Although an imbalance in power will always underpin the employer/employee relationship, from a Freirean perspective, the process of emerging from a position of disadvantage within the work environment illuminates the value of what Freire (1972) describes as the co-operative process of problematisation. By employing a problem posing approach within the employee/employer relationship, solutions are created that address both the employer and employee needs.

Supporting people with mental health disabilities to initially get, then keep work requires mental health practitioners and vocational specialists to work in tandem with their clients. These dual practitioner/client responsibilities include educating employers' about mental illness and the support systems which will promote their employee's work performance. Although the informants' experiences varied, on-the-job support was provided by most managers and in some instances, colleagues. This support included being provided with workplace accommodations. For these resources to be critical to each worker's success, these accommodations need to be tailored to each individual (Gioia, 2005; Krupa, 2004; Torrey, et al., 1998). Within his narrative, Louis describes this as a "bending in process"; employer flexibility which is enabling of worker performance as opposed to impeding. "I think its sort of like a bending in process; where you just starting building up your hours and especially if you've got trouble, well for me, losing out before you even start work. Starting small and building from there" (Louis, p. 7). In terms of the clients' day-by-day work success and satisfaction, vocational practitioners and key workers have an integral, but background role, in creating solutions which both support and facilitate the employer/employee relationship. These solutions may include developing individualised workplace accommodations that are able to flex around the individual's needs, thereby enhancing workplace success and satisfaction for people with mental illness, in order to address the issue of job retention.

\section{Interconnections with Social Networks}

Work tasks and activities engage the worker in forming connecting bonds with other people (Strong, 1998). For many of the informants, being able to see 
themselves through the eyes of colleagues and customers who represented the wider community was a confidence-building experience. In the following excerpt, Kiri is reflecting on the interrelationships between being liked and included, and being encouraged to achieve.//.. "it makes you feel that you've accomplished something, it's a good feeling being liked; it's a good feeling. I think everybody likes to be liked in someway. It encourages you to do even better" (Kiri, p. 12). This dynamic illuminates the Freirean notion of humanisation, wherein a humanly enhancing culture encourages self determination and self efficacy. While many of the informants valued the opportunity to develop connections with people, they all identified the limitations of these workplace relationships.

As described on several occasions within the findings chapters, all the informants had a circumspect and hesitant approach to their workplace relationships. Consequently, all the informants' social networks remained quite separate and apart from their work environment. This experience mirrors the finding of some mental health vocational studies, which have identified that work can potentially increase a person's sense of isolation (Becker et al., 1998; Bedell et al., 1998; Danley, 1992; Strong, 1998; Torrey et al., 1998; Wallace et al., 1999). New Zealanders affected by mental illness also agree with their North American counterparts, that working may result in the loss of supports and social contacts (New Zealand National Employment Forum, 1999).

Both the informants' experiences and the research literature reviewed for this study demonstrate how vital it is that work is interconnected with other social roles and activities, as successful employment does not guarantee satisfaction or fulfilment in other life domains (Marrone \& Golowka, 1999). Two out of nine vocational rehabilitation practise guidelines identified in a research study, were concerned with the clients' interconnections with social networks and on-the-job interpersonal issues (Torrey et al., 1998). While 'Job Clubs" are seen to provide valuable collegial pre-employment support, the informants' experiences concur with the mental health employment literature, which also suggests the value of group support after securing employment. Both practitioner-led and consumerled programmes were identified as providing valuable opportunities for 
providing both informal socialisation (Torrey et al., 1998). Blankertz and Robinson (1996) also identified the importance of maintaining support networks by providing opportunities for workers to celebrate employment achievements, using group activities such as award ceremonies, celebration dinners and weekend get-togethers. In the first instance, mental health and vocational practitioners need to be aware that the clients' employment may actually result in a loss of supports and social contacts. In the second instance, service users and practitioners need to be creatively and collaboratively responding with interventions such as after-work 'job clubs' and sourcing alternative social opportunities.

\section{Disclosure}

Within the employment context, the issues surrounding an individual's disclosure about their history of mental illness and illness-related offending are inherently complex. The informants all provided rich narrative about their actual experiences of managing both planned and unplanned disclosure. In addition, during their follow-up interviews and the member check session (as described within the methods chapter p. 67) the informants repeatedly highlighted this issue as one of their most challenging dilemmas. While there is little written about disclosure within the mental health rehabilitation literature, the information that was reviewed for this study resonates with the informants' experiences. The additional complexity of illness-related offending, however, is not reported within the New Zealand or international literature. Therefore, much of this discussion about the implications for practise relies on the informants' experiences of disclosure.

Both New Zealand and North American studies found that people who self disclose about their mental illness are usually very concerned about what it may do to their reputations and careers (Granger, 2000; Mental Health Commission, 2006; Schneider, 1998a). In situations where job accommodations are desired, or when people are concerned that their symptoms of illness may affect their job performance, Granger (2000) and Schneider (1998a) strongly recommend that workers disclose to at least one manager. In general it appears that managers deal well with the information, and that strengthens the person who has made the 
disclosure (Granger, 2000; Mental Health Commission, 2006; Schneider, 1998a). As the nagging fear of having their history of mental illness and illness-related offending exposed at work abated, the informants experienced demystification. With demystification, a concept that Freire (1972) aligns with his process of problematisation, comes a new view of reality and a founded hope for freedom.

The literature suggests a more cautious approach when disclosing to colleagues, who unlike managers, are not bound by confidentiality protocols (Granger, 2000; Krupa, 2004; Schneider, 1998a). It was evident throughout the informants' narratives that, in conjunction with their mental health and vocational practitioners', they had all carefully considered the complexities related to the issue of disclosure. Often this consideration had commenced at the time they started to contemplate re-entering the workforce. Although the informants' views differed considerably about to whom, when and what you disclose, they all concurred about the need to be thoughtful and circumspect in regard to disclosure.

The legitimacy of the prospective worker's initial and ongoing anxieties about disclosure needs to be acknowledged and addressed by their key workers and/or vocational practitioners (Granger, 2000; Schneider, 1998a). In addition to the complexities of addressing stigma and discrimination, there are legislative requirements under the New Zealand Health Information Privacy Code (1994) and the subsequent amendments (Privacy Commissioner, 2003) to consider. Therefore, the disclosure contingency plan needs to negotiated, documented and signed off by both the client and practitioner. An important component of disclosure planning is deciding the verbatim content of what will be said, who will say it, and to whom, and in what circumstances this information will be revealed (Granger, 2000; Schneider, 1998a). These conversations can be planned within group or individual meeting, in situations such as job clubs and one-onone pre-employment rehearsal sessions (Granger, 2000).

Each new job situation requires the disclosure plan to be reviewed by the mental health practitioner and the individual client. This enables the plan to be adjusted if necessary, in order to best convey the issues involved in the disclosure within that particular work culture (Granger, 2000). Discerning how to disclosure can be 
seen in terms of attempting to gain managerial or collegial buy-in for a employment-related concept that occurs outside their normal realm of experience. Using language that has meaning for the manager/colleague to whom you are trying to covey the message, appears to be the best method. Psychiatric diagnostic terms may have little or no meaning to a manager. Alternatively they may have a distorted meaning gained through fictitious dramatisation. Hence, it is useful to talk to manager in terms of experience, using language that they can comprehend and personally relate to. Mental health and vocational rehabilitation practitioners, therefore, have an important role to play in providing learning opportunities, and in facilitating the client's ability to make thoughtful choices about disclosure.

\section{Getting Going on Work Day Mornings}

All the informants in this study perceived that their medication had both aided, and impeded, their return-to-work experience. Although the informants' experience of lethargy was significant in all instances, the extent to which this sensation impacted on work performance varied from individual to individual. Therefore, in accord with the informants' narratives, management of the substantial medication-management conundrum essentially hinges on the mental health practitioner's collaboration and ability to 'hear' the client's unique lived experience. This illuminates Freire's (1972) belief that the co-operative process of meaningful dialogue is an important part of the emancipation of disenfranchised people; a sentiment that is echoed by Louis: I suppose you have to work out your own meds. Everybody's different so you got to have trust in your doctor, and your doctors have got to have trust in you as well (Louis, p. 12) Accordingly, in addition to professional observation and clinical measurement, each worker's subjective experience about the impact of their medication on their work performance needs to be viewed as a fundamental consideration when utilising medication.

\section{Wellness-at-Work Plans}

The paradigm shift towards the 'recovery approach' has seen the unique perspective of people with mental illness increasingly prevail in their illness 
management plans. Described within this thesis as wellness-at-work strategies, this term encapsulates the practical measures and intrinsic processes that most of the informants described using to self-regulate their features of illness. The informants' experiences demonstrate that it is crucial that people with mental illness have active involvement in the health care plans that support their wellness-at-work (Torrey et al., 1998). Both the informants' experiences and the international literature highlight the importance of establishing comprehensive, integrated vocational pathways which involve the individual's personal characteristics (Gioia, 2005; Killeen \& O’Day, 2004; Krupa, 2004). These pathways need to give attention to vocational exploration, job matching, job seeking and job retention skills (Torrey et al., 1998). Hence, people with mental illness require resources and supports to promote satisfaction and success within their selected work settings (Danley et al., 1992). Torrey et al. (1998) also emphasise: interconnections with social networks; and self management techniques which focus the areas of substance abuse, wellness, and on-the-job interpersonal issues.

Job stress is both a normal and often an invigorating component of the workaday world. The degree to which workplace stress impacted on the informants varied considerably. In some situations, work related tensions were alleviated by employing stress management techniques. This experience contrasts with some informants more complex anxiety-at-work situations, which required individualised, specialist interventions. The informants' collective experience of work-related fatigue highlights that this issue needs to be addressed. The remedial interventions then need to be factored into each individual's wellnessat-work plan accordingly. A number of the informants reported that being in work, and/or specific work tasks had, at times, been detrimental to their physical health, illustrating that these plans need to consider both mental health and physical well being. The management strategies the informants' described include medication, rest and supportive people. They also include employing skills such as problem solving and stress management. These are transferable skills that the informants acquired prior to employment, mainly through attending formal skills teaching sessions such as 'job clubs' and inpatient groups. 
For wellness-at-work plans to be critical to each worker's success and satisfaction, they need to be tailored to each individual (Gioia, 2005; Killeen \& O'Day, 2004; Krupa, 2004). This involvement brings with it, however, unique complexities in that these workers are people whose psychosis can present functional changes in the way an individual experiences the world. As some of the informants' narratives demonstrate, sometimes they were trying to complete work tasks while they were also attempting to organise thoughts or distinguish reality. Hence, it is important that these wellness-at-work strategies are predetermined by the workers, in collaboration with their mental health practitioner. These plans may also be developed in consultation with a nominated colleague(s) or boss(es). Depending on both the workplace culture and worker preference, however, the worker and health practitioner may decide on a more circumspect approach and just communicate select parts of the plan to nominated colleagues or managers.

These practical skills and resources were underpinned by the intrinsic processes of insight into illness and personal validation through shared experiences and mutual dialogue. The informants illustrate within their narratives Freire's (1972) belief that increased consciousness into one's situation, enables people to perceive their reality as a both a process and a transformation. As Kiri describes in the following excerpt, this awareness enables people who experience psychosis to harness these symptoms when they unexpectedly arise, thereby minimising the impact of their intrusion on work performance.

It can be quite stressful sometimes; it's not easy for people with mental illness like myself. I have to work that much harder because I think I hear things, but I just carry on. I know when it's someone else, but I know when it's my mental illness, and that sort of knowing makes it easier. If you don't know much about your mental illness, you can't do anything. (Kiri, p. 3)

Both the literature reviewed for this study and the informants' experiences illuminate Freire's (1972) notion of praxis; reflection and action being performed in a cyclical manner, enabling one to illuminate the other in order to generate a proactive response to workplace stress and early warning signs. 


\section{Creating Employment Opportunities: Socio-political Interventions}

Mental health rehabilitation practitioners may individually feel powerless to bring about socio political changes. The role of advocacy, however, is central to working in tandem with one of the most internationally disadvantaged groups in the employment sector, including the disability sector (Anthony et al., 2002; Sundar \& Ochocka, 2004). Hence, the campaign for change at the socio-political level requires rehabilitation practitioners to work with unions and the government employment sector. These bodies need to have clear policies on how the various political and managerial decision-makers will promote employment for people with disabilities. Interventions are also required to provide education, assistance and encouragement to employers and employer groups to provide equal employment opportunities and supportive work conditions for people with mental illness. The employment, health and income support sectors need to work closely together to ensure their policies and operational practises are aligned to meet the employment needs and aspirations of people with mental illness (McCrum et al., 1997).

Supporting the notion that work is an inalienable right of citizenship requires mental health practitioners and vocational specialists to work in tandem with people with mental illness, as advocates for socio-political change and as promoters of employer acceptance. The creation of this dynamic consumerprovider partnership will enable people with mental illness to make contributions to the labour market, thereby experiencing challenges and successes in the context of meaningful work. Reduction of discrimination starts with the professionals stressing the positive attributes of people who are affected by mental illness. Then the media, policy makers, elected officials and employers will have the rationale to follow suit

\section{Educational Aspects}

In contrast to North America's proactive approach, the historical absence of vocational rehabilitation strategies and practitioner training within the national New Zealand mental health operational plans clearly places vocational rehabilitation as a low priority, with minimal resources being allocated to this 
area. The New Zealand National Employment Forum (1999) identified that mental health employment services were generally ineffective in helping people find work. This combined consumer/provider forum attributed this to the majority of mental health service providers and employment specialists lacking adequate knowledge and skills, in both mental health issues and employment issues. Subsequently, the Mental Health Commission (Lapsley, 2003) stated that the vocational rehabilitation sector was not owning or addressing the challenging issue of job retention. In 2003, despite a rapidly decreasing general unemployment rate since the late nineties, significantly more New Zealanders with disabilities were unemployed (an increase of 19.5\%) and complained about discrimination than in the mid 1990s (Mintrom \& True, 2004). This acknowledges people with disabilities to be one of the most disadvantaged groups in the current New Zealand employment environment (Mintrom \& True, 2004).

New Zealand undergraduates involved in: nursing; social work; occupational therapy and community support work training programmes may pursue a career in mental health rehabilitation. Some of these students will specialise in forensic, mental health or vocational rehabilitation practise. Therefore, at both undergraduate and postgraduate education levels, mental health practitioners' training needs to incorporate vocational interventions within the rehabilitation curricula. In addition, continued on-the-job education is important for establishing and maintaining both a rehabilitative and vocational focus within forensic psychiatry. There are specialist postgraduate education opportunities available that focus on both the forensic psychiatry and the mental health rehabilitative perspective. At the time of writing this thesis, however, there was no New Zealand Qualification Authority (NZQA) accredited training programme that specifically addressed vocational rehabilitation from either a disability or mental health perspective. The move towards supported employment across the disability sector has seen a significant increase in the number of staff employed in this area (Minister for Disability Issues, 2001). As reflected in international studies, however, vocational practise may not be underpinned by a solid knowledge base (Drake et al., 1998; Schneider, 1998b). New Zealand's relatively small population base may make a generic vocational rehabilitation education 
programme, which also includes the needs of people with physical disabilities and learning disabilities, a more viable option. An education programme also creates the opportunity for the health, education and employment sectors to work closely with vocational rehabilitation providers and service users, in order to develop practise protocols that are based on evidence.

\section{Limits of the Study}

As this research was completed as a Masters thesis, the number of informants in this return-to-work study was small, although eight informants is consistent with a phenomenological research method. While unplanned, the recruiting of equal numbers of men and women from diverse cultural backgrounds, with a widerange of work roles and experiences, contributed to the depth of the findings.

The vulnerable nature of the population under study caused restrictions in utilising some of the collected narrative. This knowledge influenced my writing. At times, I experienced some tension between revealing meaning related to the informants' experiences and the need to be protective of the informants' worker role identity. As documented in the methods section of this thesis (see p. 76) workplace and specific worker role details were described in broad terms such as trade positions and food services industry. One informant also took the option of removing information about her workplace from the interview transcript prior to it being analysed.

My presuppositions and assumptions were another limiting factor in this study. There are a relatively small number of prospective informants living in New Zealand. This small cohort, combined with my lengthy experience in the field, meant some of the informants were known to me. This prior acquaintanceship may have led to people presenting information in a manner that made it more acceptable to me. In addition, both at the commencement of this study, and during it's progress, I was aware that my extensive vocational related reading and length of experience in the area of vocational rehabilitation, may influence my interpretations and preclude me from being receptive to new revelations from within the text. Prior to commencing, these limiting influences were documented as part of my preunderstandings. These influences of prior acquaintanceship and 
professional experience were also raised for scrutiny within both my personal journaling notes (as described in the methods chapter, p. 57) and within my thesis supervision sessions. I have also strived to remain cognisant of my own role within the research process, and document my own experiences within the text when it is important that the reader is cognisant of these.

Phenomenological research does not discern, or attempt to discern, absolute truths or objective observation (van Manen, 2005). Therefore, some human science researchers may be sceptical of the rigour and depth of this study. The confirmability of a study, however, is judged by the adequacy of information leading to the reader's ability to audit the researcher's influences and actions throughout the entire research process (Koch, 1996; Polit et al., 2001). As described within the methods chapter, I have aimed to diligently record both my data analysis, and the accompanying rationale, in a detailed well referenced format in order to add to the rigour and depth of the data analysis.

My phenomenological writing sought to reveal the meanings contained within the text, thereby evoking insights and understandings. As with all such writing, there are limits to fully capturing the phenomenon in question: "In point of fact, all interpretive phenomenological inquiry is cognisant of the realisation that no interpretation is ever complete, no explication of meaning is ever final, no insight is beyond challenge" (van Manen, 2005, p. 7). There are the inherent limits of written language to consider, as well as the enigmatic nature of the text. My abilities as a writer were also subject to inherent constraints when I sought to fully capture the essence of the informants' narratives, and then portray these in a textual form that allowed the meanings to be revealed to the reader. The intuitive process, however, is part and parcel of phenomenological research. Responding to both the opportunities and constraints of phenomenological writing was an element of my self reflection that occurred throughout this study. Ultimately, this deliberation and reflection brought about a deeper awareness of the return-towork phenomenon. 
Suggestions for Future Research

The assessment of risk in relation to the probability of people with a forensic history re-offending continues to be the subject to extensive research (Lindqvist $\&$ Skipworth, 2000). In contrast, authors writing from a rehabilitation perspective concur that there is scant reference to rehabilitation within the forensic psychiatry literature (Couldrick \& Aldred, 2003; Tracey, 2000). From a mental health vocational rehabilitation standpoint, forensic psychiatry is scarcely mentioned in the existing literature (Lloyd, 1995). There is an increasing professional requirement for practise to be validated by evidence. Therefore, the deficit of vocational and rehabilitation research in relation to the forensic psychiatry, is of concern. Studies that focus on both rehabilitative and vocational interventions within a forensic context would start to address the gap in the forensic psychiatry literature. In particular, qualitative studies that focus on the forensic service-users' lived experience would be a valued addition to the forensic psychiatry body of knowledge, because at present these are unexplored (Couldrick \& Aldred, 2003). This would allow forensic service-users' unique perceptions of what constitutes meaningful rehabilitation, to also contribute to the body of knowledge.

A key factor facing rehabilitation staff working in forensic psychiatry is the challenge of reconciling the tensions between a rehabilitative and risk management approach (Couldrick \& Aldred, 2003). These contradictions and challenges apply at all levels of secure care, however, are particularly brought into question when clients have moved back into the community. With this shift, forensic and vocational rehabilitation practitioners have to navigate through the frequently contradictory goals of social inclusion; treatment and public safety. When mutually considering the choice of work placement, forensic and vocational rehabilitation practitioners have to consider and balance potential risk, and the public perception of risk, against the client's rights to access opportunities that are equal to other people. Therefore, a study that reveals forensic and vocational rehabilitation practitioners' experience of the return-towork phenomenon would be a valuable addition to the scope of this return-towork inquiry. This may be a prospective opening for a doctoral thesis. 
The different ways in which the men and women in this study had established their work ethos and skills, was the only distinct gender difference identified during the analysis. This finding serves to provide insight into the political and historical influences on gender work roles, which are now well acknowledged within feminist and general New Zealand employment literature (Mintrom and True, 2004). The breadth and complexity of this historical reality precluded further examination within this text, although the profoundly influencing nature of gender work role influences, both historical and present, invite further dedicated research from a disability and work perspective.

\section{Concluding Summary}

The overall vision for this study was to complete an in-depth analysis of what it is like to re-enter the workforce following long-term forensic hospitalisation. Accordingly, the critical hermeneutic design enabled a detailed exploration of the phenomenon in terms of power relationships, and through the multiple positionings of psychiatric disability, employment status and social capital. Freire's (1972) critical social theory also served to add meaning and critical depth to the findings. This research process, therefore, evoked insights and developed understandings about how people who have a history of mental illness and illness-related offending, go about seeking, securing and sustaining employment.

The importance of providing opportunities for people with mental illness to have a voice within research is an underpinning tenet of a critical hermeneutic design. Hence, it is fitting that the conclusion of this thesis includes the words of some of the informants. All the informants revealed an aspect of the return-to-work experience that was unique, with some disparate views serving to add depth to the understandings. There were also many aspects of the return-to-work experience that resonated with all the informants.

I think I just said to myself, I just want to get my life together, prove that I can lead a good life, and having a job is an extra bonus on that one. (Louis, p. 9) 
So I started work with nothing really, but what I did have, and that probably prompted me along, was that I wanted to re-enter the world that my family and friends were living in. (Maria, p. 15)

The whole experience of work is scary, but I must say I got one good thing out of it though, and that's knowing I can actually can go and work. I think that's why my brother and my father got a bit of pride in me at the end. (Joe, p. 19)

Rather than relying on a single voice, the above compilation of narratives highlight the informants' collective experience of returning to work, and with fortitude and support from others, regaining their self and family pride by realising their career and work aspirations.

Within the mental health vocational rehabilitation literature, forensic psychiatry is scarcely mentioned in the existing body of knowledge. From the forensic psychiatry standpoint, there is scant reference to rehabilitation in the related literature. Therefore, I hope that that this study will be a timely contribution, and that the findings can serve a useful purpose by informing forensic rehabilitation and vocational rehabilitation practise. This study may also elicit further discussion and analysis regarding work and education outcomes, from the unique and important forensic psychiatric rehabilitation perspective. 


\title{
APPENDICES
}

\author{
Appendix A
}

\section{Participant Information Sheet}

\begin{abstract}
Project Title: The experiences of people who re-enter the workforce following discharge from a forensic setting.

\section{Invitation}

You are invited to participate in this study which aims to look at the experience of returning to work following discharge from a Forensic hospital. Because of your own experience of getting a job, you may be interested in hearing more about being in this study. Please read this sheet, and if you think you may like to take part, please contact me by phoning $x x x x x$, or writing to me at $x x x x x$. Or you could let the person who contacted you know of your interest and they'll pass that on to me.
\end{abstract}

\section{What is the purpose of the study?}

This study is part of the requirements for my Masters degree. During the first part of this degree, I studied about mental health rehabilitation. Because of the work I do; helping people with mental illness get back into work opportunities, during my studies I have paid special attention to what's happening in the area of employment. Unfortunately, not many studies about this area have been done in New Zealand. Therefore, this study will be a close- up look at a group of Kiwi's experience of getting a job. Because there is often even more at stake after spending time in a forensic hospital, this study also aims to look at how you experienced other people's (e.g. employers) and other services' (e.g. WINZ) response to you getting back into work. Hopefully, together we can discover how your experiences can help us understand the situation better. From this deeper understanding, we may be able to help improve the experience for others in a similar position, who want to get back into the workforce. There is also a good possibility that some of what we discover may benefit you, the next time you are job hunting.

\section{How are people chosen to be asked to be part of the study?}

If you have had the experience of returning to work after being in hospital for longer than three years, I would like to talk with you. You will also need to be living in your own place for at least six months (or perhaps with your family, or friends, or other residents in supported accommodation) in Auckland, or North of Auckland.

While you may, or may not, be employed right now, in order for us to have a good look at how the experience was for you, you'll have needed to have stayed with the job for at least six months.

\section{What happens in the study?}

We will get together in a quiet, private place to talk about how it was for you when you went back to work. Together we will choose a private, convenient place and time to meet. This may be in your own home. Alternatively, we could also get together at my office in Avondale, which just has the street number on it and a sign saying "Work Foundations", so it's quite low key and apart from other health services. I would organise your transport there and back home again. You may also like to bring a friend or other support people with you.

During our conversation about work, which will take about 1 to $1 \frac{1 / 2}{2}$ hours, I will ask you some opening lines to get you talking, such as "tell me a story about when you returned to work". The questions are not hard or tricky, and I will give you some prompts if you run out of steam, or if I need more information to be really clear about your story. After 


\section{Appendix A (cont.)}

our first interview, we will get back together again a month or so later, so you can check out if your typed-up story feels 'right'. There may be points you wish to make clearer at this time. During both meetings, I will need to use a tape recorder, and a typist will turn our conversation into type- written pages we can look at together.

This study is about your experience of returning to work. I will not focus on, or ask questions about your other life or mental health experience, although you may choose to raise some of these issues during our discussion.

\section{What are the discomforts and risks?}

Talking about going back to work may bring up some difficult feelings for you, particularly if the experience wasn't so good. You may also get so caught up in the story; you may tell me things you later regret. You can let me know if this happens, and I will remove these things from your story without questioning your reasons why.

Because this study may include some of the comments you've made, someone who knows you very well may recognise bits of your story in the brief snippets used to make a point about returning to work. Together, we will make sure that your workplace isn't able to be recognised.

\section{How will these discomforts and risks be alleviated?}

Before we start our first meeting, we will both choose a person who you can talk to; if you feel you need to at any time during this study. This may be the person who first told you about this study, or another key worker you feel comfortable with.

I will also ring and check in with you the day after the interview. If you do feel stirred up by talking about your work experience and you feel like it would be a good move, there are three free counselling sessions available to you at AUT, the University which is supervising this study.

Together, we will check the information to make sure your current or past employer(s) are not able to be recognised. This also applies to any other identifiable life events, including the reasons why you came to be in a Forensic hospital. I also have a supervisor who will help us with this, because it is important that being part of this study doesn't make any workplace issues harder for you.

\section{What the benefits?}

I hope that by looking at your return- to- work, that we may discover some steps that may improve the experience for others wanting to get a job. As returning to living and working independence after such a major life disruption, is still quite new in New Zealand, you will have the opportunity to allow others to benefit from your experiences.

There is also a good possibility that some of what we discover may benefit you, the next time you are job hunting.

\section{What compensation is available for injury or negligence?}

I plan to consider your well- being at all stages of this study. AUT has three free counselling sessions available for you, if you require them.

If you do have any concerns about the nature of the study, or about how it is managed, please contact my supervisor, who's details are at the bottom of this form, or the Health Advocates Trust ph 0800555050.

\section{How will my privacy be protected?}

You will be asked to choose another name for yourself which will be used when writing up your story. You may want to also think about the option of using a unisex name, as this can also give you additional privacy by not identifying you as either a man or a woman e.g. Tracey, Kerry etc. 


\section{Appendix A (cont.)}

Choosing a private place and time to be interviewed will also help to keep your privacy. The typist will sign a confidentially agreement so the taped information will be kept private.

All your information will be stored in a locked cabinet, in a locked building. After the study, this information has to be locked away and stored for ten years, and then it is destroyed by shredding. Up to the final stages of putting all the information together for marking, if you don't want to continue in the study, your information can be destroyed immediately.

As I said before in this sheet, some information snippets may be recognised by people who know you well, so we cannot be totally anonymous. However, keeping your privacy in the wider community is very important. Together we will check the information to make sure your employer is not able to be recognised, and my supervisor will also help us with this.

\section{What are the costs of participating in the project? (including time)}

The main cost of being part of this study is your time, which is valuable. Therefore we will plan these times to suit you and your household. We will need to spend one, to one and half hours together, both times we meet.

If you need transport to and from these interviews, I will organise it or pay for a taxi.

\section{Opportunity to consider invitation}

Please think about this information, and perhaps talk about it some more with the person who gave you this sheet. It is entirely voluntary, and you should not feel any pressure to participate. You can leave the study and request all your information is destroyed or returned to you, up to when I finish collecting all the stories.

If you are interested in finding out more about this research, or in being part of this study, you can ring me on XXxxx. I really look forward to hearing from you by March 31 2005 (this is just after Easter), if you are still interested in sharing your story after reading this information.

\section{Opportunity to receive feedback on results of research}

After our second meeting, I will finish analysing the information I have collected. Before I put this in for marking, I will give you an opportunity to hear about the results. Everyone who contributed their story to this research will have an opportunity to say how the information will be shared. Together, we will need to identify the right places and the people that we have confidence in, to help us make a difference.

\section{Participant Concerns}

Any concerns regarding the nature of this project should be directed in the initially to the Project Supervisors Dr. Jo Walton ph. Xxxxx or Kirk Reed ph. Xxxxx

Concerns regarding the conduct of the research should be directed to the Executive Secretary, AUTEC, Madeline Banda, madeline.banda@aut.ac.nz , ph xxxxx

Researcher Contact Details: Jenni Tregoweth; Ph xxxxx

Project Supervisor Contact Details: Dr. Jo Walton, Professor, AUT, ph xxxxx Kirk Reed, Senior Lecturer, AUT, ph xxxxx. 
Appendix B

\section{Consent to Participation in Research}

This form is to be completed in conjunction with, and after reference to, the AUTEC Guidelines

(Revised January 2003).

Title of Project: The experiences of people who re-enter the workforce following discharge from a forensic setting.

Project Supervisors: Dr. Jo Walton

Kirk Reed

Researcher: Jenni Tregoweth

I have read and understood the information provided about this research project (Information Sheet dated 13.12.04: Version 2).

- I understand that the interview will be audio-taped and transcribed.

- I understand that I can request my audiotapes are returned to me following the completion of the study.

- I understand that I may withdraw myself or any information that I have provided for this project at any time prior to completion of data collection, without being disadvantaged in any way.

- If I withdraw, I understand that all relevant tapes and transcripts, or parts thereof, will be returned to me if requested, or alternatively destroyed.

- I agree to take part in this research.

- I wish to receive a copy of the report from the research.

Participant signature:

Participant name:

Participant Contact Details (if appropriate):

Date:

Note: The Participant should retain a copy of this form.

Health and Disability Ethics Committee: reference number: AKY/04/11/303 


\title{
Appendix C
}

\section{Statement by a Relative/ Friend/ Family/ Whanau}

\author{
Lay Title: The experiences of people who re-enter the workforce \\ following discharge from a forensic setting.
}

Principal Investigator: Jenni Tregoweth

Participant's Name:

I have read and I understand the information sheet dated 14.09.04 for people taking part in the study designed to explore the experiences of people who re-enter the workforce following discharge from a forensic setting. I have had the opportunity to discuss this study. I am satisfied with the answers I have been given.

I understand that taking part in this study is voluntary and that my relative/friend may withdraw from the study at any time if he/she wishes. This will not affect his/her continuing health care.

I understand that his/her participation in this study is confidential and that no material which could identify him/her will be used in any reports on this study.

I know whom to contact if my relative/friend has any concerns arising from the study or if anything occurs which I think he/she would consider a reason to withdraw from the study.

This study has been given ethical approval by the Ethics Committee. This means that the Committee may check at any time that the study is following appropriate ethical procedures.

I would like a copy of the results of the study.

\section{YES/NO}

Signed:

Date

Printed Name:

Relationship to Participant:

Address for results: 
Appendix D

\section{Typist Confidentiality Agreement}

Title of Project: The experiences of re entering the workforce following discharge from a forensic setting.

\section{Project Supervisors: Dr. Jo Walton}

Kirk Reed

Researcher:

Jenni Tregoweth

I understand that all the material I will be asked to transcribe is confidential. I understand that the contents of the tapes can only be discussed with the researchers. I will not keep any copies of the transcripts nor allow third parties access to them while the work is in progress.

Typist's signature:

Typist's name:

Typist's Contact Details:

Date:

Project Supervisor Contact Details:

Health and Disability Ethics Committee: reference number; AKY/04/11/303 
Appendix E

\section{Indicative Questions}

The following are examples of the types of questions that the informants were asked. As each interview progressed questions were tailored toward each person's individual experience of their return-to-work. Probing questions to follow up on points were brief and neutral e.g. "how is that?"

- Tell me as much of the story about your return-to-work, as you feel able to

- Which experiences were most helpful for returning to work?

- Which experiences made your return-to-work more difficult?

- Tell me about the things that were particularly significant to you in your new work place?

- Looking back, which life experiences have best prepared you for getting a job?

- You may think of something else you would like to say in relation to this interview. I'll turn back on the tape if you think of something now. If it comes to you later, jot it down or ring me and let me know. 
Appendix F

Interview Framework: The Critical Perspective

\begin{tabular}{|c|c|c|c|c|}
\hline \multicolumn{5}{|c|}{ Dr. E. Marion Jones (2000). Capital as Power } \\
\hline Economic: & Political & Cultural & Social & Symbolic \\
\hline $\begin{array}{l}\text { - Wealth } \\
\text { and } \\
\text { income } \\
\text { - Goods and } \\
\text { services } \\
\text { - } \quad \text { Budgets }\end{array}$ & $\begin{array}{l}\text { - } \text { Position } \\
\text { - } \text { Ranking } \\
\text { - } \text { Credibility } \\
\text { - } \\
\text { - } \\
\text { Paw }\end{array}$ & \begin{tabular}{|ll} 
& Status \\
- & Beliefs \\
- & Attitudes \\
& Educational \\
& credentials \\
- & Aesthetic \\
& preferences \\
- & Results of \\
& socialization \\
- & Inherited \\
& traits \\
\end{tabular} & $\begin{array}{ll}\text { - } & \text { Network } \\
\text { - } & \text { Family } \\
\text { - } & \text { Teams }\end{array}$ & $\begin{array}{ll}\text { - } & \text { Communication } \\
\text { social } \\
\text { differentiation } \\
\text { legitimizes }\end{array}$ \\
\hline \multicolumn{5}{|c|}{ Elizabeth Heilman (2003). Levels of Power } \\
\hline \multicolumn{2}{|c|}{ MACRO } & \multirow{2}{*}{$\begin{array}{l}\text { CULTURAL } \\
\text { "Language, } \\
\text { texts and } \\
\text { curriculum". }\end{array}$} & \multicolumn{2}{|c|}{ MICRO } \\
\hline \multicolumn{2}{|c|}{$\begin{array}{l}\text { "Political and economic } \\
\text { structures". }\end{array}$} & & \multicolumn{2}{|c|}{$\begin{array}{l}\text { "Immediate experiences of } \\
\text { freedom, possibility, control, } \\
\text { and oppression". }\end{array}$} \\
\hline \multicolumn{5}{|c|}{$\begin{array}{l}\text { Jenni Tregoweth (2005). Return-to-work: The critical lens underpinning the } \\
\text { hermeneutic interviews. } \\
\text { Examples of interview content to frame into person-specific examples to } \\
\text { accompany questions and to focus on as interview prompts. }\end{array}$} \\
\hline \multicolumn{2}{|c|}{$\begin{array}{l}\text { Mental health and criminal } \\
\text { justice sections: impact on } \\
\text { employment. } \\
\text { Economics; transferring from } \\
\text { a benefit. } \\
\text { Economics: impact of wages } \\
\text { on residential fees; housing } \\
\text { corp. rent. } \\
\text { - Affirmative/ lack of } \\
\text { affirmative policies by } \\
\text { government departments re: } \\
\text { return-to-work. } \\
\text { Barriers to work presented by } \\
\text { the mental health; WINZ; } \\
\text { employment government } \\
\text { sectors. }\end{array}$} & \begin{tabular}{|ll} 
- & $\begin{array}{l}\text { Education } \\
\text { credentials }\end{array}$ \\
- & Stigma and \\
& discriminatio \\
& n. \\
- & Workplace \\
& values \\
- & Work \\
& organization' \\
s affirmative \\
policies
\end{tabular} & \multicolumn{2}{|c|}{$\begin{array}{l}\text { Alienation in the workplace } \\
\text { - Socialization with colleagues in } \\
\text { and outside of work. } \\
\text { Assignment of generally } \\
\text { undesired, low ranking duties. } \\
\text { - Workplace respect and courtesy; } \\
\text { from both bosses and colleagues. } \\
\text { Ability and opportunity to 'ask' } \\
\text { and make requests of bosses/ } \\
\text { colleagues. }\end{array}$} \\
\hline
\end{tabular}




\author{
Appendix G \\ Letter to the Intermediaries
}

Dear

Thank you for your offer to help with my research project: "The experiences of people who re-enter the workforce following discharge from a Forensic setting" Your perspectives on the subject will be valuable throughout the development of this project.

I now have documented ethical approval from the Regional Health Ethics Committee, which means I can embark on interviewing research participants. Enclosed with this letter is a participant information sheet which explains my research to prospective participants in detail. One of the ethical issues regarding this research is that I need to use intermediaries to make the initial approach to my participants. This is because many people are known to me, and I need to be sure they don't feel coerced into participation because of this.

I believe you have a collegial relationship with a person who is prospective participants for my research. Additionally, you have demonstrated an interest in my research topic. Hence I would like you to be an intermediary for introducing my research topic to people who fulfil my participant criteria as follows:

It is proposed to recruit both men and women who are either currently employed, or unemployed. The sample will include Maori and others who have had the following experiences:

- They were hospitalised within a forensic setting for three years or longer within the last 15 years.

- They have now been discharged from a forensic inpatient setting for at least six months, and are living in their own, or in a family living situation, or in a community based supported accommodation.

- Within the last decade, they have returned to part or full time employment for a period of at least six months, and received tax related earnings as remuneration.

Within this intermediary role, I need you to inform the prospective participant to enable them to have an initial broad understanding of the research project, and following that, see if they agree to have a further no-obligation conversation with me about the research. I will explain the project and methods I will use in-depth during this meeting with the prospective participant, and they will then make their decision on whether or not, to participate. The prospective participants will be encouraged to consult with support people and family members regarding their potential participation.

Some important points to stress in your initial conversation are as follows: 
- That the research is just an interview conversation with me, which will be audio taped. There are no written questionnaires or other surveys to complete i.e. it is entirely a verbal account of their personal return to work experience.

- The interview will be one to one and half hours long, and take place in a private location of their choosing e.g. their own home. I'll also need to do a follow up interview with them after I have the initial interview transcripts back, just to check that they feel OK about the information and see if they want to remove/ explain anything they may have said.

- The interview will focus on their return-to-work experiences. I will not be asking any questions which relate to other life experiences or request other personal information that is not directly relevant to the return- towork experience.

- There will be a koha in terms of vouchers e.g. petrol, Whitcoulls, Farmers etc depending on what they desire.

- It will be confidential interview and research document. We will not identify them by name or use any identifying features i.e. work place details. Women may also choose a pseudonym that is unisex like Sam or Terry so they are less identifiable.

All this information is detailed more fully in the enclosed participant information sheet.

Thank you for considering my request for you to adopt an intermediary role with regards to my research. Please let me know if you require any more additional information. I look forward to the opportunity of working with you on this project, and hopefully it will enable us both to understand our clients' return-towork experience better. If you have any further queries please phone me on $\mathrm{ph}$. 8201580 .

Jenni Tregoweth 
Appendix $\mathrm{H}$

Letter to the informants regarding the member check session

\subsection{6 .06}

Dear

Last year, you spoke to me about your return-to-work experiences during an audio taped interview. Since that time, I've worked with all the material I gathered from your and the other participants' interviews to write up this research. My aim was to place all of your return-to-work descriptions into themes, in a way that best reflects your experiences. I have enclosed these themes with this lunch invitation. Not everyone experienced all the themes, and some of the experiences were very different, for different people. However, there were number of themes that everyone had a story about. Some of these include 'Persevering against the Odds' and 'Work as an Elixir'.

In order to talk to you more about these themes and hear your thoughts about my findings, you are invited to a lunch on:

\section{Sunday July 9 at midday.}

\section{Place:}

Some of you may choose to talk to me on your own because attending this forum will mean meeting the other people who took part in the study, and you may wish to keep your participation confidential. Alternatively, you may just want a written summary when I've completed the project near the end of the year. During this informal lunch, your individual contributions made during your interviews will not be identified as being yours. You will also have your travel expenses reimbursed.

If you can come, please let me know ph $x x x x x$, or you can just turn up if you make your decision at the last moment, I just need some idea for catering. Once again, thanks for contributing to this study. Your experiences will greatly assist the process of people moving back into employment.

Best wishes

Jenni Tregoweth 


\section{REFERENCES}

American Psychiatric Association. (2000). Diagnostic statistical manual of mental health disorders ( ${ }^{\text {th }}$ ed.). Arlington, VA: American Psychiatric Association.

Ansley, B. (1999). The future of work. The New Zealand Listener. September 4, 14-18.

Anthony, W. (1994). Characteristics of people with psychiatric disabilities that are predictive of entry into the rehabilitation process and successful employment. Psychosocial Rehabilitation Journal, 17(3), 3-13.

Anthony, W., Cohen, M., \& Farkas, M. (1992). Psychiatric rehabilitation. Boston, MA: Boston University, Center for Psychiatric Rehabilitation.

Anthony, W., Cohen, M., Farkas, M., \& Cagne, C. (2002). Psychiatric rehabilitation $\left(2^{\text {nd }}\right.$ ed.). Boston, MA: Boston University, Center for Psychiatric Rehabilitation.

Anthony, W., Rogers, S., Cohen, M., \& Davies, R. (1995). Relationship between psychiatric symptomatology, work skills and future vocational performance. Psychiatric Services, 46(4), 353-358.

Association for Supported Employment in New Zealand. (1996). Jobs for New Zealanders with disabilities: Prepared as a discussion document by Association for Supported Employment in New Zealand. Levin, NZ: Omega Publications.

Auerbach, E., \& Richardson, P. (2005). The long-term experiences of persons with severe and persistent mental illness. Psychiatric Rehabilitation Journal, 28(3), 267-273.

Baron, R., \& Granger, B. (1997). Employment for people with psychiatric disabilities: Knowledge dissemination utilisation experiences. New Directions for Mental Health, 74, 67-77.

Baronov, D. (2004). Conceptual foundations of social research. Boulder, Colorado: Paradigm Publishers.

Becker, D., \& Drake, R. (1994). Individual placement and support: A community mental health center approach to vocational rehabilitation. Community Mental Health Journal, 30(2), 193-206.

Becker, D.R., Drake, R.E., Bond, G.R., Xie, H., Bradley, D.J., \& Harrison, K. (1998). Job terminations among people with severe mental illness participating in supported employment. Community Mental Health Journal. 34(1), 71-80 
Becker, D.R., Drake, R.E., Farabaugh, A., \& Bond, G.R. (1996). Job preferences of clients with severe psychiatric disorders participating in supported employment programmes. Psychiatric Services, 47(11), 1223-1226.

Becker, D., Drake, R.E., \& Naughton, W.J. (2005). Supported employment for people with co-occurring disorders. Psychiatric Rehabilitation Journal, 28(4), 332-338.

Bedell, J., Draving, D., Parrish, A., Gervey, A., Gervey, R., \& Guastadisegni, P. (1998). Description and comparison of experiences of people with mental disorders in supported employment and paid prevocational training. Psychiatric Rehabilitation Journal, 21(3), 279-283.

Blankertz, L., \& Robinson, S. (1996). Adding a vocational focus to mental health rehabilitation. Psychiatric Services, 47(11), 1216-1222.

Bond, G. (1998). Principles of the individual placement and support model: Empirical support. Psychiatric Rehabilitation Journal, 22(1), 11-23.

Bond, G. (2004). Supported employment: Evidence for an evidenced based practise. Psychiatric Rehabilitation Journal, 27(4), 345-359.

Bond, G., Drake, R., Mueser, K., \& Becker, D. (1997). An update on supported employment for people with severe mental illness. Psychiatric Services, 48(3), 335-346.

Bozzer, M., Samson, D., \& Anson, J. (1999). An evaluation of a community based vocational rehabilitation program for adults with psychiatric disabilities. Canadian Journal of Community Mental Health, 18(1), 16578 .

Bryson, G., Bell, M., Greig, T., \& Kaplan, E. (1999). The work behaviour inventory: prediction of future work success of people with schizophrenia. Psychiatric Rehabilitation Journal, 23(2), 113-116.

Burns, N., \& Grove, S. (1997). The practise of nursing research $\left(3^{\text {rd }}\right.$ ed.). Philadelphia: W.B. Saunders Company.

Bybee, D., Mowbray, C.T., \& McCrohan, N. M. (1996). Towards zero exclusion in vocational opportunities for persons with psychiatric disabilities: Prediction of service receipt in a hybrid vocational/ case management service program. Psychiatric Rehabilitation, 19(4), 15-27.

Chandler, D., Levin, S., \& Barry, P. (1999). The menu approach to employment services: Philosophy and five-year outcomes. Psychiatric Rehabilitation Journal, 23(1), 24-33.

Chandler, D., Meisel, J., Hu, T., McGowen, M., \& Madison, K. (1997). A capitated model for a cross section of severely mentally ill clients: 
Employment outcomes. Community Mental Health Journal, 33(6), 501516.

Chaplow, D., Chaplow R., \& Maniapoto, W. (1993). Addressing cultural differences in institutions: Changing health practices in New Zealand. Criminal Behaviour and Mental Health, 3, 307-321.

Christie, W. (1997). Treaty Issues. Christchurch: Wyvern Press.

Corrigan, P., \& Garman, A. (1997). Considerations for research on consumer empowerment and psychosocial interventions. Psychiatric Services, 48(3), 347-352.

Couldrick, L., \& Aldred, D. (2003). Forensic Occupational Therapy. London: Whurr.

Crotty, M. (1998). The foundations of social research: Meaning and perspective in the research process. St. Leonards, Australia: Allen \& Unwin.

Danley, K. S., Sciarappa, K., \& MacDonald W. (1992). Choose-Get- Keep: A psychiatric rehabilitation approach to supported employment. New Directions for Mental Health Services, 53, 67-76.

Day, H., \& Alon, E. (1993). Work, leisure and quality of life for vocational rehabilitation consumers. Canadian Journal of Rehabilitation, 7(2), 119125.

Deegan, P. (1992). The independent living movement and people with psychiatric disabilities: Taking back control over our own lives. Psychosocial Rehabilitation Journal, 15(3), 3-19.

Denzin, N., \& Lincoln, Y. (1994). Handbook of qualitative research. Thousand Oakes, California: Sage.

Department of Labour: Te Tari Mahi. (2006). Labour market reports. Employment \& Unemployment. Retrieved September 21, 2006, from http://www.dol.govt.nz/publications/lmr/lmr-HLFS.asp

Depoy, E., \& Gitlin, L. (1998). Introduction to research: Understanding and applying multiple strategies $\left(2^{\text {nd }}\right.$ ed.). St Louis: Mosby.

Diamond, H. (1998). Vocational decision-making in a psychiatric outpatient program. Occupational Therapy in Mental Health. 14(3), 67-80.

Dorio, J. (2004). Tying it all together- the PASS to success. Psychiatric Rehabilitation Journal, 28(1), 32-39.

Drake, R.E., Becker, D.R., Biesanz, J.C., Torrey, W.C., McHugo, G. J., \& Wyzik, P.F. (1994). Rehabilitative day treatment vs. supported 
employment: I vocational outcomes. Community Mental Health Journal. 30(5), 519-532.

Drake, R.E., Fox, T. S., Leather, P.K., Becker, D.R., Musumeci, J.S., Ingram, W.F., \& McHugo, G. J. (1998). Regional variation in competitive employment for persons with severe mental illness. Administration and Policy in Mental Health, 25(5), 493-504.

Durie, M. (1998). Whaiora: Maori health development ( $2^{\text {nd }}$ ed.). Auckland: Oxford University Press.

Durie, M. (2001). Te mana, Te kawanatanga. Auckland: Oxford University Press.

Eikelmann, B., \& Reker, T. (1993). The second labour market? Vocational rehabilitation and work integration of chronically mentally ill people in Germany. Acta Psychiatrica Scandinavica, 88,124-129.

Ezersky, S. (1991). The flower workshop: Cultivating prevocational skills. Mental Health Special Interest Section of the American Occupational Therapy Association, Inc, 14, 1-2.

Farkas, M. (1996). Advances in psychiatric rehabilitation: North America. In C. Vazquez \& J. Aldaz (Eds.), Advances in Psychiatric Rehabilitation (pp. 23-26). Madrid, Spain: Siglo XXI.

Frame, J. (1992). The pocket mirror. Auckland: Vintage.

Freire, P. (1972). Pedagogy of the oppressed. London, Great Britain: Penguin Books.

Freire, P. (2004). Pedagogy of hope. New York: Continuum Publishing Company.

Garske, G. (1999). The challenges of rehabilitation counsellors: Working with people with psychiatric disabilities. Journal of Rehabilitation, 65(1), 215.

Gervey, R., \& Kowal, R. (1995). Job development strategies for placing persons with psychiatric disabilities into supported employment jobs in a large city. Psychosocial Rehabilitation Journal, 18(4), 95-113.

Gioia, D. (2005). Career development in schizophrenia: A heuristic framework. Community Mental Health Journal, 41(3), 307-323.

Granerud, A., \& Severinsson, E. (2006). The struggle for social integration in the community: The experiences of people with mental health problems. Journal of Psychiatric and Mental Health Nursing, 13(3), 288-293. 
Granger, B. (2000). The role of psychiatric rehabilitation practitioners in assisting people understand how best to assert their ADA rights and arrange job accommodations. Psychiatric Rehabilitation Journal, 23(3), 215-223.

Grant, B., \& Giddings, L. (2002). Making sense of methodologies: A paradigm framework for the novice researcher. Contemporary Nursing, 13, 10-28.

Harris, C. (1995). Barriers to employment for mental health consumers. Paper presented at the 1st Work Rehabilitation Symposium, Auckland University of Technology: Auckland.

Health Funding Authority. (2000). Striking a better balance: A funding response to reducing inequalities in health. Wellington: Government Printer.

Heaney, T. (1995). Issues in Freirean Pedagogy. Thresholds in Education. Retrieved September 8, 2003, from http://nlu.edu/ace/Resources/Documents/FreireIssues.html

Heilman, E. (2003). Critical theory as a personal project: From early idealism to academic reality. Educational Theory. Urbana, 53(3), 247-266.

Huberman, A. M., \& Miles, M. B. (1998). Data Management and analysis methods. In N. Denzin \& Y. Lincoln, Collecting and interpreting qualitative materials (pp. 179-199). Thousand Oakes, California: Sage.

Joe, C. (2003). The development of community forensic occupational therapy. In L. Couldrick \& D. Aldred, Forensic occupational therapy (pp. 133-145). London: Whurr.

Jones, E. M. (2000). Shaping team practise in the context of health reform. Unpublished doctoral thesis. Flinders University of South Australia, Adelaide, Australia.

Jungersen, K. (2002). Cultural safety: Kawa Whakaruruhau- An occupational therapy perspective. New Zealand Journal of Occupational Therapy, 49(1), 4-9.

Killeen, M.B., \& O’Day, B.L. (2004). Challenging expectations: How individuals with psychiatric disabilities find and keep work. Psychiatric Rehabilitation Journal, 28(2), 157-163.

King, R., Wahorn, G., Lloyd, C., McLeod, P., McMah, T., \& Leong, C. (2006). Enhancing employment services for people with severe mental illness: The challenge of the Australian employment service. Australian and New Zealand Journal of Psychiatry, 40, 471-477.

Klos, R. (2005). Response to open letter by Robyn Stent. Occupational Therapy Insight, April, 7. 
Koch, T. (1996). Implementation of a hermeneutic inquiry in nursing: Philosophy, rigour and representation. Journal of Advanced Nursing, 24(1), 174-184.

Kristiansson, M., Sumelius, K., \& Sondergaard, H. (2004). Post-traumatic stress disorder in forensic psychiatric setting. The Journal of the American Academy of Psychiatry and the Law, 32(4), 399-407.

Krupa, T. (2004). Employment, recovery and schizophrenia: Integrating health and disorders at work. Psychiatric Rehabilitation Journal, 28(1), 8-12.

Krupa, T., McCourty, K., Bonner, D., Von Briesen, B., \& Scott, R. (2000). Voices, opportunities \& choices employment club: Transforming sheltered workshops using an affirmative business approach. Canadian Journal of Community Mental Health, 18(2), 11-21.

Lapsley, H. (2003). Disability in employment: Where is government strategy heading. Occasional paper number one. Wellington: Mental Health Commission.

Lehman, A. F. (1995). Vocational rehabilitation in schizophrenia. Schizophrenia Bulletin, 2, 645-656.

Lette, E. (1992). The stigmatised patient. In P. J. Fink \& A. Tasman (Eds.), Stigma and Mental Illness (pp. 17-25). Washington DC: American Psychiatric Press.

Lincoln, Y., \& Guba, E. (1985). Naturalistic inquiry. In D. Polit, C. Beck, $\&$ B. Hungler, Essentials of nursing research: Methods, appraisals and utilisation (5th ed.) (pp. 312-316). Philadelphia, PA: Lippincott.

Lindqvist, P., \& Skipworth, J. (2000). Evidenced-based rehabilitation in forensic psychiatry. British Journal of Psychiatry, 176, 320-323.

Lloyd, C. (1995). Trends in forensic psychiatry. British Journal of Occupational Therapy, 58(5), 209-213.

Macaskill, D. (2000). Household labour force survey: June 2000 quarter. Statistics New Zealand fOnlmel. Retrieved March 21, 2001, from http/Avww.stats.govtnz; File: Cat 01.500 Set 00/01-011.

Maeve, M., \& Vaughan, M. (2001). Nursing with prisoners: The practise of caring, forensic nursing or penal harm. Advances in Nursing Science, 24(2), 47-64.

Mallik, K., Reeves, R., \& Dellario, D. (1998). Barriers to community integration for people with severe and persistent psychiatric disabilities. Psychiatric Rehabilitation Journal, 22(2), 175-180. 
Marrone, J., \& Golowka, E. (1999). If work makes people with mental illness sick, what do unemployment, poverty, and social isolation cause? Psychiatric Rehabilitation Journal, 23(2), 187-193.

Mason, K. (1988). Report of the committee inquiry into procedures used in certain psychiatric hospitals in relation to admission, discharge or release and leave of certain classes of patient. Wellington: Government Printer.

McCrum, B., Burnside, L., \& Duffy, T. (1997). Organising for work: A job clinic for people with mental health needs. Journal of Mental Health, 6 , 503-513.

McGurrin, M. (1994). An overview of the effectiveness of traditional vocational rehabilitation services in the treatment of long term mental illness. Psychosocial Rehabilitation Journal, 17(3), 37-54.

McHugo, G.J.; Drake, R.E., \& Becker, D.R. (1998). The durability of supported employment effects. Psychiatric Rehabilitation Journal, 22(1), 55-61.

Mental Health Commission. (1998). Blueprint for mental health services in New Zealand: How things need to be. Wellington: Government Printer.

Mental Health Commission. (1999). Employment in mental health: Issues and opportunities. Wellington: Government Printer.

Mental Health Commission. (2006). Self-disclosure benefits explored. Like Minds, Like Mine, 25, 4.

Michon, H.W.C., Kroon, H., van Weeghel, J., \& Schene, A.H. (2004). The generic work behaviour questionnaire (GWBQ) assessment of core dimensions of generic work behaviour of people with severe mental illness in vocational rehabilitation. Psychiatric Rehabilitation Journal, $28(1), 40-47$.

Midgely, G. (1990). The social context of vocational rehabilitation for ex-psychiatric patients. British Journal of Psychiatry, 156, 272-277.

Minister for Disability Issues. (2001). The New Zealand disability strategy: Making of world of difference whakanui oranga. Wellington: Government Printer.

Ministry of Health: Manatu Houora. (1997). The National mental health standards. Wellington: Government Printer.

Ministry for Social Development. (2001). Pathways to social inclusion/Nga ara whakauuru kite whanui: Improving vocational direction for people with disabilities. Wellington: Government Printer. 
Mintrom, M., \& True, J. (2004). Framework for the future: Equal employment opportunities in New Zealand. Unpublished report produced for the Equal Opportunities Unit of the Human Rights Commission. University of Auckland, Auckland, New Zealand.

Mitchell, D.P., Betts, A., \& Epling, M. (2002). Youth employment, mental health and substance abuse: a challenge to mental health services. Journal of Psychiatric and Mental Health Nursing, 9, 191- 198.

Monahan, J. (1992). Mental health disorders and violent behaviour. American Psychologist, 47, 511-521.

Mueller, H. H., \& Wilgosh L. (1991). Employment survival skills: Frequency and seriousness of skill deficit occurrences for job losses. Canadian Journal of Rehabilitation, 4(4), 213-226.

National Health Committee. (1998). The social, cultural and economic determinants of health in New Zealand: Actions to improve health. Wellington: Government Printer.

Neuman, L. (2003). Social research methods: Qualitative and quantitative approaches $\left(5^{\text {th }}\right.$ ed.). Boston: Allyn and Bacon.

New Zealand National Mental Health Forum. (1999). Real jobs for real pay: Report on the First National Employment Forum to identify opportunities and obstacles facing people who experience mental illness. Wellington: Association for Supported Employment in New Zealand.

O'Neill, D.M., \& Bertollo D. N. (1998). Work and earnings losses due to mental illness: Perspectives from three national surveys. Administration and Policy in Mental Health, 25(5), 505- 523.

Orrin, D. (1997). Consumer involvement in policy making. Psychiatric Rehabilitation Journal, 21(1), 75-79.

Polit, D., Beck, C., \& Hungler, B. (2001). Essentials of nursing research: Methods, appraisals and utilisation (5th ed.). Philadelphia, PA: Lippincott.

Powers, P. (2003). Empowerment as treatment and the role of health professional. .Advances in Nursing Science, 26(3), 227-237.

Pozner, A., Ng, M., Hammond, J., \& Shepherd, G. (1996). Working it out: Creating work opportunities for people with mental health problems: A development handbook. Brighton, Great Britain: Pavilion Publishing Ltd.

Pratt, C.W., Gill, K.J., Barrett, N.M., \& Roberts, M.M. (1998). Psychiatric Rehabilitation. San Diego: Academic.

Privacy Commissioner. (2003). Health information privacy code 1994: 
Incorporating amendments and including revised commentary.

Wellington: Government Printer.

Public Service Association. (2004). What is the future of work? The Future of Work. Retrieved June 30, 2004, from

http://www.psa.org.nz/futurework.asp

Read, J. (2003). Emancipation songs: Individual participation by service users in mental health care. Occasional paper number two. Wellington: Mental Health Commission.

Reddon, J.R., McNeil, D.C., Schmitke, M.I., \& Trelenberg, L. A. (1992). Patient evaluation of a psychiatric hospital's vocational rehabilitation program. Canadian Journal of Rehabilitation, 6(2), 111-115.

Reker, T., Hornung, W., Schonauer, B., \& Eikelmann, B. (2000). Long term psychiatric patients in vocational rehabilitation programmes: A naturalistic follow-up study over three years. Acta Psychiatrica Scandinavica, 101, 457- 463.

Rogers, S., Anthony, W., Cohen, M., \& Davis, R. (1997). Prediction of vocational outcome based on clinical and demographic indicators among vocationally ready clients. Community Mental Health Journal, 33(2), 99112

Rogers, S., \& Palmer-Erbs, V. (1994). Participatory action research: Implications for research and evaluation in psychiatric rehabilitation. Psychosocial Rehabilitation Journal, 18(2), 4-12.

Ryan, P.M. (1993). The revised dictionary of modern Māori. Auckland: Heinemann Education, Reed Publishing Group.

Salzer, M. (1997). Consumer empowerment in mental health organisations: Concept, benefit, and impediments. Administration and Policy in Mental Health, 24, 425- 434.

Sarantakos, S. (1994). Social research. Melbourne, Australia: Macmillan Education.

Sayce, L. (2000). Mainstreaming mental health. Open Mind, the Mental Health Magazines, 102(Mar/Apri), 13.

Schneider, D. (1998a). When do I disclose? ADA protection and your job. Occupational Therapy in Mental Health, 14(1/2), 77-87.

Schneider, J. (1998b). Work interventions in mental health care: Some arguments and recent evidence. Journal of Mental Health, 7(1), 81-94.

Schneider, Z., Elliott, D., LoBiondo-Wood, G., \& Harber, J. (2003). Nursing research: Methods, critical appraisal and utilisation ( $\left.2^{\text {nd }} \mathrm{ed}.\right)$.

Marrickville, NSW: Mosby. 
Schofield, R. (1998). Empowerment education for individuals with serious mental illness. Journal of Psychosocial Nursing, 36(11), 35-40.

Simpson, A., Jones, R., Evans, C., \& McKenna, B. (2006). Outcomes of patients rehabilitated through a forensic psychiatry service: A 7.5 year retrospective study. Behavioural Sciences and the Law, 24, 833-843.

Simpson, A., McKenna, B., Moskowitz, A., Skipworth, J., \& Barry-Walsh, J. (2003). Myth and reality: The relationship between mental illness and homicide in New Zealand. Auckland: Health Research Council.

Smellie, P. (2006). It's scary: Don't debate it. Like Minds, Like Mine, 25, 4.

Smith, B. (1998). The problem drinker's lived experience of suffering: An exploration using hermeneutic phenomenology. Journal of Advanced Nursing, 27, 213-222.

Smith, M.K. (2002). Paulo Freire and informal education. infed.org. Retrieved September 8, 2003, from http://www.infed.org/thinkers/et-freir.htm.

Smythe, L. (2000). Making sense of qualitative research. Kai tiaki Nursing New Zealand, 16-18.

Spence, A. (2004). The job cure. The New Zealand Listener, July 24, 26-28.

Stent, R. (2005). Open letter to members of parliament in relation to the repeal of the disabled person's employment promotion (DPEP) Act. Occupational Therapy Insight, April, 7.

Strong, S. (1998). Meaningful work in supportive environments: Experiences with the recovery process. The American Journal of Occupational Therapy, 52(1), 31- 38 .

Sundar, P., \& Ochocka, J. (2004). Bridging the gap between dreams and realities related to employment and mental health: Implications for policy and practice. Canadian Journal of Community Mental Health, 23(1), 75- 89.

Swanson, J., Borum, R., Swartz, M., \& Hiday, V. (1999). Violent behaviour preceding hospitalisation among persons with severe mental illness. Law and Human Behaviour, 23(2), 185-203.

Swartz, M.S., Swanson, J.W., Hiday, V.A., Borum, R., Wagner, R., \& Burns, B. (1998).Violence and severe mental illness: The effects of substance abuse and nonadherance to medication. American Journal of Psychiatry, 155(2). 226-231.

Taylor, P., \& Gunn, J. (1999). Homicides by people with mental illness: Myth and reality. British Journal of Psychiatry, 174, 9-14. 
The Royal Commission on Social Policy. (1988). Work: It's nature role and value in New Zealand: Discussion Booklet Three. Wellington: Government Printer.

Tolich, M., \& Davidson, C. (1999). Starting fieldwork: An introduction to qualitative research in New Zealand. Auckland: Oxford University Press.

Torrey, W., Bebout, R., Kline, J., Becker, D. R., Alverston, M., \& Drake, R. (1998). Practise guidelines for clinicians working in programmes providing integrated vocational and clinical services for persons with severe mental illness. Psychiatric Rehabilitation Journal, 2(4), 388-393.

Torrey, W.C., Mueser, K.T., McHugo, G.H., \& Drake, R.E. (2000). Self esteem as an outcome measure in studies of vocational rehabilitation for adults with severe mental illness. Psychiatric Services, 51(2), 229-233.

Tracey, F. (2000). Time to discover. Mentally disordered offenders: Their experience of rehabilitation in forensic psychiatry. Unpublished masters thesis. Auckland University of Technology, Auckland, New Zealand.

Tsang, H. (2000). Predictors of employment outcome for people with psychiatric disabilities: A review of the literature since the mid 80s. Journal of Rehabilitation, 66(2), 19-30.

Van Dongen, C. (1996). Quality of life and self-esteem in working and non working persons with mental illness. Community Mental Health Journal, $32(6), 535-548$.

Vangergang, A. (1996). Consumer/ survivor participation in the operation of community mental health agencies and programs in metro Toronto: Input or impact. Canadian Journal of Community Mental Health, 15, 153-158.

van Manen, M. (1997). Researching lived experience: Human science for an action sensitive pedagogy ( $2 \mathrm{nd}$ ed.). London, Ontario: The Althouse Press.

van Manen, M. (2005). Writing in the dark: Phenomenological studies in interpretive inquiry. London, Ontario: The Althouse Press.

Vostanis, P. (1990). The role of work in psychiatric rehabilitation: A review of the literature. British Journal of Occupational Therapy, 53(1), 24-28.

Wallace, C. (1993). Psychiatric rehabilitation. Psychopharmacology Bulletin, 29(4), 537- 548.

Wallace, C. J., Tauber, R., \& Wilde, J. (1999). Teaching fundamental workplace skills to persons with serious mental illness. Psychiatric Services, 50(8), 1147-1153. 
Warner, R., \& Polak, P. (1995). The economic advancement of the mentally ill in the community, 2: Economic choices and disincentive. Community Mental Health Journal, 31(5), 477-492.

Warriner, R. (1993). Supported employment: An approach to the vocational rehabilitation of psychiatric consumers. New Zealand Mental Health News, Autumn, 25-26.

Wehman, P. H., Revell, G., Kregal, J., Kreutzer, J., Callahan, M., \& Banks, D. (1991). Supported employment: An alternative model for vocational rehabilitation of persons with severe neurologic, psychiatric, or physical disability. Arch Physical Medical Rehabilitation, 72, 101-105.

Wilgosh, L. (1990). Organisational climate and workers with mental disabilities, Canadian Journal of Rehabilitation. 4(1), 9-16. 